Nadia Lamamra

Le genre de l'apprentissage, lapprentissage du genre Quand les arrêts prématurés révèlent les logiques à l'œuvre en formation professionnelle initiale 
Nadia Lamamra

Le genre de l'apprentissage, l'apprentissage du genre

Quand les arrêts prématurés révèlent les logiques à l'œuvre en formation professionnelle initiale 
La collection scientifique "Contributions à la recherche en formation professionnelle", sous la direction de l'Institut fédéral des hautes études en formation professionnelle EHB IFFP IUFFP, porte sur les questions actuelles relatives à la formation professionnelle. Elle offre un éclairage multidisciplinaire (relevant notamment des sciences de l'éducation, de l'économie, de la psychologie et de la sociologie) et est également ouverte à d'autres perspectives de recherche. Les publications sont évaluées et contribuent à enrichir le débat scientifique ainsi qu'à développer la formation professionnelle.

\section{Comité d'éditeurs}

Prof. Dr Carmen Baumeler, Dr Antje Barabasch, Dr Isabelle Caprani, Prof. Dr Jürg Schweri

Eidgenössisches Hochschulinstitut für Berufsbildung EHB / Institut fédéral des hautes études en formation professionnelle IFFP / Istituto Universitario Federale per la Formazione Professionale IUFFP 
Contributions à la recherche en formation professionnelle

Nadia Lamamra

Le genre de l'apprentissage, l'apprentissage du genre

Quand les arrêts

prématurés révèlent

les logiques à l'œuvre

en formation professionnelle initiale 
Publié par

Éditions Seismo, Sciences sociales et questions de société SA, Zurich et Genève www.editions-seismo.ch | info@editions-seismo.ch

La maison d'Édition Seismo bénéficie d'un soutien de

l'Office fédéral de la culture pour les années 2021-2024.

Texte @ l'auteure 2021

ISBN 978-2-88351-073-9 (Print 2016)

ISBN 978-2-88351-744-8 (PDF)

https://doi.org/10.33058/seismo.20744

Couverture: Hannah Traber, St Gall

Cet ouvrage est couvert par une licence Creative Commons

Attribution - Pas d'Utilisation Commerciale - Pas de Modification 4.0

International (CC BY-NC-ND 4.0) 


\section{Table des matières}

$\begin{array}{ll}\text { Remerciements } & 7\end{array}$

Préface Prisca Kergoat 9

$\begin{array}{ll}\text { Introduction } & 13\end{array}$

Chapitre 1

Les inégalités de sexe en formation professionnelle en Suisse

$\begin{array}{lll}\text { 1.1 Regard rétrospectif sur la mise en place du système suisse } & 19\end{array}$ de formation professionnelle

1.2 Un système de formation lié au marché de l'emploi 22

1.3 La transition école-travail: un enjeu central de la 32 formation professionnelle

1.4 La formation professionnelle, un espace avec ses logiques propres 36

Chapitre 2

Le genre: angle mort de la littérature sur les arrêts en formation professionnelle

2.1 La littérature sur les arrêts prématurés en formation professionnelle duale

2.2 Portrait sociographique des jeunes qui arrêtent leur formation professionnelle

Chapitre 3

Soumettre la formation professionnelle à une analyse de genre

3.1 Genre, rapports sociaux de sexe, système de genre: quels concepts pour traduire la réalité de la formation professionnelle?

3.2 De l'école obligatoire à la formation professionnelle: socialisations et orientations

3.3 La question des pionnières et des pionniers

Chapitre 4

\section{Problématique et méthodologie}

4.1 Une perspective décentrée

4.2 Une analyse secondaire des données

4.3 Apports et limites d'une analyse secondaire 
Chapitre 5

Arrêter sa formation professionnelle: motifs et processus 97

5.1 L'impossibilité d'apprendre le métier 98

$\begin{array}{lll}5.2 & \text { Des relations de travail difficiles } & 108\end{array}$

$\begin{array}{lll}5.3 & \text { Les contraintes du monde du travail } & 125\end{array}$

$\begin{array}{lll}5.4 & \text { Des problèmes de transition } & 133\end{array}$

$\begin{array}{lll}5.5 & 139\end{array}$

$\begin{array}{lll}5.6 & \text { Le genre comme processus transversal } & 141\end{array}$

Chapitre 6

Le passage en formation professionnelle: une expérience 147 de la souffrance au travail?

6.1 La souffrance en formation professionnelle 148

$\begin{array}{lll}6.2 & \text { Un contact douloureux avec le travail } & 151\end{array}$

6.3 A l'origine de la souffrance, la division sexuelle 175 du travail

Chapitre 7

Des stratégies pour faire face à la souffrance $\quad 179$

7.1 Souffrance des apprenti·e.s et réponses spécifiques 181

$\begin{array}{lll}7.2 & \text { Les défenses mises en œuvre } & 187\end{array}$

$\begin{array}{lll}7.3 & \text { Des stratégies révélatrices du statut d'apprenti.e } 202\end{array}$

7.4 Derrières les stratégies défensives, des enjeux multiples 215

Chapitre 8

La formation professionnelle: un lieu de fabrication $\quad 220$ du genre

8.1 Apprentissage de la division sexuelle du travail et de la hiérarchie 223 entre les sexes

8.2 Socialisation aux normes de genre en formation professionnelle 232

8.3 Des apprentissages qui soulignent la complexité des rapports 248 de genre

8.4 L'apprentissage, une socialisation aux diverses facettes du 253 système de genre

Conclusion

Références bibliographiques $\quad 269$

$\begin{array}{ll}\text { Annexes } & 288\end{array}$ 


\section{Remerciements}

Cet ouvrage est la version remaniée d'une thèse de doctorat en sciences sociales qui a été soutenue à l'Université de Lausanne en septembre 2011.

Un grand merci à Patricia Roux pour sa confiance et son accompagnement dans ce travail. Par ses remarques, elle m’a fait apprécier de remettre sans cesse l'ouvrage sur le métier. Cette collaboration a été pour moi riche à bien des égards.

Mes remerciements vont également aux membres de mon jury, Nicky Le Feuvre, Pascale Molinier et Gilles Moreau pour leurs commentaires, leurs suggestions et leurs critiques. Ces premiers échanges m’ont permis d'affiner mes analyses et surtout de construire des collaborations dont j'apprécie chaque jour la richesse.

Je suis extrêmement reconnaissante à Jacqueline De Puy et Magdalena Rosende, amies et collègues, pour leur écoute et confiance, leurs encouragements, relectures et suggestions à divers moments de ce travail.

Patrick Auderset m'a apporté une aide inestimable en faisant preuve d'un soutien indéfectible dès la première esquisse. Il a largement contribué à transformer des résultats de recherche en un véritable livre.

Je tiens à remercier Lucienne Chevalier pour sa relecture finale de l'ouvrage. Ses suggestions ont largement contribuéà en améliorer la tenue.

Merci encore à mes collègues de l'IFFP, en particulier Barbara Duc, Marine Jordan, Letizia Saugy et Jürg Schweri, pour leurs encouragements, leur patience et leur soutien.

J'adresse mes remerciements aux ami.e.s, dont mille petits gestes m'ont rappelé quotidiennement le soutien, si précieux dans les moments de doute.

Cette recherche a été rendue possible grâce au soutien de l'Institut fédéral des hautes études en formation professionnelle. En finançant ce projet à l'interne, l'IFFP m'a donné l'occasion de mener une recherche approfondie sur plusieurs années. Ce faisant, j’ai pu bénéficier des conditions pour travailler dans une relative sérénité et m’atteler à ce travail de doctorat.

Ma reconnaissance va encore à l'association Transition école métier - TEM, en particulier à Svend Lehmann, Fabio Lecci et Catherine Kaltenrieder, pour leur aide précieuse dans l'accès au terrain et les échanges stimulants sur les premières pistes d'analyse. 
Et enfin, j'aimerais remercier les jeunes qui ont accepté de me parler de leur vie, de leur expérience en formation professionnelle à une période souvent difficile. Elles et ils mont permis de mieux saisir leur situation, de comprendre leurs difficultés, mais aussi leurs joies. 


\section{Préface}

\section{Prisca Kergoat}

Maîtresse de conférences, Université Jean-Jaurès, Toulouse II, CERTOP (Centre d'étude et de recherche Travail, Organisation, Pouvoir), UMR 5044 Céreq (Centre d'études et de recherches sur les qualifications)

C'est une entrée qui pourrait sembler étroite que celle de la rupture des contrats d'apprentissage dans le canton de Vaud. Mais qu'on ne s'y trompe pas... Partant de son terrain, la recherche de Nadia Lamamra invite à pénétrer bien des univers : celui des jeunesses adolescentes, du travail, des emplois d'exécution et des groupes du bas du salariat tout en démontrant, et c'est sa force, la transversalité du système de genre.

Cet ouvrage permet d'alimenter, et c'est son premier mérite, les connaissances d'un continent peu connu, celui de la production et de la reproduction de l'ordre de genre au sein de la formation professionnelle. En effet, non seulement ce segment du système éducatif reste relativement méconnu - et c'est particulièrement vrai en France -, mais de plus l'emprise du genre sur la formation professionnelle s'avère très peu explorée. Mobilisant une importante revue de la littérature issue de différentes disciplines et sous disciplines (sociologie del'éducation, sociologie du travail et psychodynamique du travail), l'auteure défend l'idée selon laquelle «le genre traverse l'entier de l'expérience de la formation professionnelle, y compris le processus qui conduit à l'interrompre» (p. 69). Elle démontre ainsi que la division sexuelle du travail organise tant l'apprentissage en acte (la répartition des tâches et les rapports hiérarchiques) que ce qui s'y passe en amont (les modalités d'entrée en apprentissage dont les choix d'orientation) et en aval (l'insertion professionnelle et les conditions d'emploi). La formation professionnelle apparait donc bel et bien comme une "fabrique du genre»: les apprenti.e.s font ensemble (dans les professions mixtes) ou séparément (dans des professions fortement sexuées) l'expérience de la division sexuelle du travail. Filles et garçons ne se confrontent pas uniquement à des savoirs de métier, ils doivent également se conformer à leur position asymétrique dans les rapports sociaux de sexe, incorporer les normes de féminité et de masculinité, se soumettre à la hiérarchie entre les groupes de sexe et au système hétéronormatif. 
La perspective défendue, celle conduisant à affirmer que le système de formation suisse ne peut être analysé qu'en relation étroite avec le marché du travail, s'avère heuristique. Tout d'abord elle permet de donner sens et forme à la configuration des filières de formation: sur-sélection des filles pour l'accès à une place d'apprentissage, absence de mixité, persistance des bastions masculins et concentration des filles sur quelques filières de formation alors même que ces dernières connaissent une meilleure réussite scolaire que les garçons. Ensuite, l'examen des processus de socialisation, de la manière dont ils produisent et traduisent le genre, montre combien filles et garçons sont encouragé.e.s, voire forcé.e.s, à adopter un comportement jugé adapté à leur sexe. La démonstration de l'auteure conduit ainsi à renforcer la thèse selon laquelle ce n'est pas parce que garçons et filles préparent des formations différentes que le marché du travail est ségrégué, mais bel et bien parce que le marché du travail est profondément inégalitaire que l'un et l'autre sexe se confrontent à des expériences différenciées. La formation professionnelle ne se contente pas d'enregistrer et de reproduire les inégalités construites dans la famille et à l'école, elle contribue, aux côtés du marché du travail, à la production des ghettos féminins, à la sexuation des qualifications, des compétences comme des plafonds de verre.

Parmi les nombreux apports de cet ouvrage, je souhaite souligner la façon dont, à travers les récits recueillis, l'auteure révèle l'existence d'un univers méconnu celui des adolescent.e.s au travail. La situation de travail, l'immersion en entreprise mènent à se confronter précocement à une position, celle de future travailleur ou travailleuse d'exécution mais aussi à un statut particulier, celui de l'apprenti.e occupant le bas de l'échelle hiérarchique. Les récits des 46 apprenti.e.s, âgé.e.s de 15 ans pour les plus jeunes d'entre eux, témoignent tour à tour de l'expérience de domination, d'humiliation et de discrimination. Le lecteur découvre un monde évoquant les temps anciens, celle de la figure du mousse très prisée dans la littérature pour décrire les violences infligées aux enfants, figure encore utilisée, dans le petit Larousse, à la définition de souffre-douleur. L'ouvrage de Nadia Lamamra permet d'interpeller les pouvoirs publics sur des pratiques tant individuelles que collectives qui ne devraient plus avoir cours. Depuis longtemps dénoncées par la JOC, ces violences relatées par l'auteure font écho au scandale qui a fait suite aux témoignages de nombreux chefs cuisiniers français sur les coups, les humiliations, le harcèlement sexuel et l'ambiance particulièrement misogyne des cuisines. 
Venons-en maintenant à un résultat qui, sur la question des expériences apprenties, mérite de retenir notre attention. J'insisterai, pour ma part, sur trois dimensions particulièrement stimulantes. En premier lieu, l'auteure montre comment elles et ils - indépendamment de leur sexe - endossent les tâches socialement assignées aux femmes (travail d'entretien, morcellement du travail, disponibilité permanente). Al'instar de l'armée, c'est en destituant les garçons des avantages attachés à leur sexe que les collectifs de travail rappellent que pour être inséré au sein d'un collectif de travail il faut d'abord être un «homme» et faire preuve de sa virilité. En second lieu, et en miroir de la dimension précédente, l'analyse du vécu des pionnières, de celles qui inversent les habitudes de genre et intègrent les bastions masculins, confirme la littérature existante quant aux brimades d'ordre psychologique, sexuel et parfois même physique auxquelles ces dernières sont trop souvent confrontées. Mais à la différence des garçons, ces pratiques visent non pas à les intégrer mais à les exclure durablement des collectifs de travail afin de préserver une communauté d'hommes. Une troisième dimension, celle de la souffrance éthique, empruntée à la psychodynamique du travail et appliquée aux expériences apprenties, s'avère particulièrement heuristique. En effet, parce qu'apprenti.e mais aussi parce qu'adolescent.e.s elles et ils n'ont que peu droit à la parole et doivent composer avec des pratiques patronales parfois déloyales, frauduleuses voire malhonnêtes qui contribuent à un fort désenchantement envers le monde du travail et envers celui des adultes. Pour faire face à la souffrance et aux contraintes des situations de travail, les apprenti.e.s déploient des stratégies identiques mais leurs mises en mots traduisent des différences de genre: la stratégie du retrait est pour les pionnières une réponse aux manifestations d'hostilité du collectif de travail alors que pour les garçons, insérés dans des métiers féminisés, le retrait a davantage à voir avec le refus des bas salaire et des faibles perspectives de carrière.

L'analyse de la souffrance au travail conduit, et c'est un résultat important de l'ouvrage, à opérer une mise à distance avec une littérature scientifique qui postule premièrement que la rupture de contrat trouverait son origine dans les caractéristiques individuelles ou dans l'histoire singulière des apprenti.e.s et deuxièmement que les symptômes observés, suite à l'arrêt de la formation (perte de motivation, désengagement, baisse des performances scolaires), seraient à analyser comme la conséquence d'un échec, celui de la rupture du contrat d’apprentissage. L'analyse menée par Nadia Lamamra nous invite à faire un pas de côté: la rupture de contrat et les symptômes associés sont le produit de la 
souffrance rencontrée en situation de travail, souffrance induite par des pratiques collectives. L'arrêt de formation prend dès lors une toute autre signification, loin d'être réduit à une défaite individuelle, il doit aussi être compris comme une volonté d'émancipation, une stratégie défensive permettant de rompre avec des situations de disqualification voire de maltraitance. Elle permet notamment aux filles de reconsidérer leur choix de formation et de choisir une formation plus exigeante correspondant davantage à leur niveau comme à leurs performances scolaires.

Pour terminer, je souhaite saluer l'exercice auquel se prête l'auteure. L'entrée par les deux dimensions que sont la formation professionnelle d'une part et le genre d'autre part, conduit à dépasser les frontières académiques. Parce qu'en rapport étroit avec l'école, le monde du travail et des entreprises, mais aussi parce que la formation professionnelle contribue à produire et à reproduire la division sexuelle du travail, l'auteure s'emploie à faire apparaître les chaines d'interdépendance entre différents champs ou sphères d'activités que sont la sphère éducative, la sphère productive et la sphère reproductive. Ces schèmes d'analyse, difficiles à mettre en œuvre et se devant d'être réinventés selon les objets soumis à investigation, nécessitent d'user de ficelles de métier, de tours de main, de toutes les qualités dont l'auteure a fait preuve en fabriquant ce «bel ouvrage».

Là ne sont pas ses moindres qualités. Riche et dense, il est également servi par une écriture à la fois claire et vivante, construit sur une démonstration rigoureuse et étayé par des récits souvent émouvants. 


\section{Introduction}

La formation en Suisse depuis l'après-guerre a connu une évolution sans précédent. On observe une tendance àl'élévation du niveau de formation tant chez les femmes que chez les hommes. Toutefois, cette évolution est plus marquée chez les femmes, qui ont «rattrapé leur retard» en matière de formation post-obligatoire, que ce soit au secondaire II ou au tertiaire ${ }^{1}$. Ainsi, parmi les 25-34 ans, la part de femmes avec une formation d'une haute école spécialisée ou universitaire est plus élevée que celle des hommes (OFS, 2015).

Malgré ce changement de fonds, des différences marquées persistent. Ainsi, si elles se sont largement approprié le niveau secondaire II, les femmes sont plus nombreuses dans les formations générales, les hommes étant davantage investis en formation professionnelle. Autre aspect qui demeure quasi inchangé : la sexuation des filières de formation et des métiers. En effet, le choix de la profession ou du domaine d'études est toujours très marqué par l'appartenance à un groupe de sexe. La ségrégation horizontale reste marquée: des formations et des métiers féminins d'une part, des formations et des métiers masculins d'autre part. La mixité des filières de formation ou des métiers reste l'exception. Le clivage hommes-femmes dans le choix de la formation s'est légèrement atténué, ce notamment par les changements de comportements féminins. On constate ainsi que la part des femmes dans des formations traditionnellement masculines s'est légèrement accrue, même si l'on parle toujours de pionnières tant l'entrée dans ces «bastions» demeure difficile. Le mouvement inverse (entrée plus grande des hommes dans des formations typiquement féminines) reste cependant timide.

En ce qui concerne l'emploi, les différences entre les sexes sont manifestes. Les taux d'activité, la situation dansla profession, les salaires, mais aussi les domaines d'activité (conséquence directe de la formation

1 En Suisse, le système éducatif est organisé selon les degrés suivant: tout d'abord, la scolarité primaire, suivie par le secondaire. Ces deux degrés constituent le cursus de scolarité obligatoire. Le post-obligatoire est également appelé Secondaire II, il peut être professionnel (apprentissage) ou académique (gymnase, collège). Enfin, le supérieur (académique ou professionnel) est également appelé Tertiaire. Il regroupe les Hautes écoles universitaires, spécialisées, les écoles polytechniques fédérales, mais aussi les brevets, diplômes et maîtrises professionnels. 
suivie) et l'évolution dans la profession sont façonnées par l'appartenance de sexe. A ce titre, la Suisse demeure l'un des pays occidentaux où la ségrégation (horizontale ou verticale) et où les discriminations sont les plus marquées entre femmes et hommes. Cette situation a de nombreuses conséquences sur l'accès à des formations continues, l'accès aux prestations des assurances sociales ou encore la prise en charge du travail domestique.

La formation professionnelle occupe une position particulière au carrefour entre formation et emploi. Elle s'inscrit dans le prolongement de la scolarité obligatoire, mais en est distincte puisqu'elle est régie au niveau fédéral ${ }^{2}$. Par ailleurs, elle est intrinsèquement liée au marché du travail, notamment dans sa forme duale, puisque les entreprises participent pleinement à la formation. Cette position particulière en fait un lieu privilégié où observer les mécanismes de production et de reproduction des inégalités entre femmes et hommes. En effet, elle perpétue des inégalités construites en amont dans l'univers scolaire (curriculum caché, orientation) et anticipe les logiques de ségrégation et de discrimination du marché du travail (sélection, inégalités). Mais la formation professionnelle n'est pas uniquement un lieu de perpétuation ou d'anticipation d'inégalités produites dans d'autres espaces, c'est aussi un lieu soumis à des mécanismes propres. En effet, la formation professionnelle initiale est un espace qui rompt avec la relative mixité scolaire que les jeunes ont connue au secondaire (les formations sont sexuées comme les métiers auxquels elles préparent). Par ailleurs, l'enjeu étant de "produire de futur.e.s travailleurs et travailleuses pour un marché ségrégué, la formation professionnelle est le lieu d'un processus de socialisation sexuée et d'une (re)négociation avec les normes de genre (arrangements, stratégies, résistances, reconfigurations)» (Lamamra, Fassa Recrosio et Chaponnière, 2014).

Par ailleurs, la formation professionnelle reste aujourd'hui encore la voie de formation post-obligatoire privilégiée. Les données récentes évaluent que plus des deux-tiers des jeunes qui quittentl'école obligatoire, débutent l'enseignement post-obligatoire (secondaire II) en formation professionnelle (SEFRI, 2015). Le choix massif de cette filière est le résultat d'un processus historique, qui a débuté à la fin du XIX ${ }^{\mathrm{e}}$ siècle et

2 En Suisse, le système de formation est du ressort des cantons, exception faite de la formation professionnelle, qui est régie par une Loi fédérale (Loi sur la formation professionnelle, LFPr). Sa mise en œuvre est de la responsabilité conjointe de la Confédération, des Cantons et des associations professionnelles (SEFRI, 2015). 
a pris son essor durant la période de plein emploi des Trente Glorieuses (1945-1975). Ainsi, les représentations qui entourent le système de formation professionnelle et son diplôme - le Certificat fédéral de Capacités (CFC) - se construisent durant cette période d'essor économique. Le CFC est fortement valorisé et ce par l'ensemble des acteurs sociaux (institutions, entreprises, parents et jeunes) ${ }^{3}$. En Suisse, ce diplôme est fortement associé à l'insertion professionnelle, puisqu'il permet d'entreprendre le métier appris ou d'accéder à d'autres activités professionnelles. En effet, le CFC a longtemps été le diplôme requis pour attester d'un niveau de qualification et ce indépendamment de la branche dans laquelle il a été obtenu. Les changements qui sont intervenus ces dernières années ont toutefois ébranlé cette «centralité» du CFC (Lamamra et Moreau, 2016).

$\mathrm{Au}$ vu de l'intérêt suscité par cette filière de formation ${ }^{4}$, mais aussi de son importance dans le paysage de la formation helvétique, et enfin de sa position au carrefour entre formation et marché du travail, il semble indispensable de procéder à une analyse approfondie sous l'angle du genre, encore balbutiante à ce jour ${ }^{5}$. En effet, il s'agit de mieux comprendre la manière dont les mécanismes de discriminations à l'œuvre sur le marché du travail agissent en formation professionnelle. C'est en posant un regard rétrospectif sur la constitution de cette filière, mais également en l'analysant comme véritable anti-chambre du marché du travail, en observant les liens très étroits qui unissent ces deux espaces, que l'on pourra saisir quelques-uns des enjeux sous l'angle du genre (Chapitre $1^{\mathrm{er}}$ ). Pour mener à bien cette réflexion, une perspective originale a été adoptée. Il s'agit de porter un regard décentré sur le système de formation professionnelle, à partir des arrêts prématurés qui s'y déroulent (Chapitre 4). Le postulat, pour cette approche décentrée, est que les situations d'arrêts - en ce qu'elles constituent des moments de

3 Ce plébiscite est plus grand du côté alémanique où le taux de jeunes optant pour la formation professionnelle est plus important qu'en Suisse romande, où une plus grande proportion s'oriente vers une formation générale (écoles de culture générale ou gymnases).

4 Lors de sa visite en Suisse en avril 2015, le président français François Hollande a souligné son intérêt pour le système suisse de formation professionnelle. En juillet 2015, lors de sa visite aux Etats-Unis, le ministre suisse de l'économie Johann Schneider-Ammann a vanté le système de formation professionnelle comme étant la clé de la réussite helvétique.

5 On notera, pour la Suisse, les travaux de Imdorf $(2004,2007 a, 2007 b)$, portant principalement sur les pratiques de discrimination à l'embauche des PME et, pour la France, l'ouvrage de Depoilly (2014) qui s'inscrit davantage dans une tradition de sociologie de l'éducation, puisqu'analysant le lycée professionnel (soit la formation professionnelle en école plein-temps). 
crise pour les personnes concernées, mais également pour le système permettent d'observer des mécanismes fins, qui se donneraient alors plus facilement à voir. Partant de ce postulat, la littérature sur les arrêts prématurés sera soumise à une analyse de genre (Chapitre 2). La position de la formation professionnelle entre éducation et travail soulève également une question théorique. En effet, la littérature en sociologie de la formation professionnelle est relativement rare (Imdorf, Granato, Moreau et Waardenburg, 2010). Il s'agit dès lors de l'analyser en empruntant les outils de la sociologie de l'éducation et de la sociologie du travail, mais également en recourant aux travaux sur les choix d'orientation (Chapitre 3). Procéder à une analyse de genre d'un objet dont les contours sont aussi peu définis nécessite de questionner les notions utilisées, notamment celles de genre et celle de rapports sociaux de sexe (Chapitre 3). Cette analyse abordera ensuite différents aspects liés aux arrêts prématurés et à ce qu'ils révèlent des logiques de genre. Les raisons invoquées pour expliquer l'arrêt seront l'occasion de faire apparaître l'ambivalence fondatrice de la formation professionnelle (Moreau, 2003), à savoir une tension entre deux logiques opposées: la formation et la production. Derrière ces raisons, se cachent des réalités professionnelles distinctes (liées à la ségrégation professionnelle) et des façons d'investir les deux pôles de la formation professionnelle sexuées (Chapitre 5). La question de la souffrance en formation professionnelle laissera apparaître les parades de genre des jeunes lors des entretiens, mais mettra à nouveau en évidence les différentes réalités professionnelles de métiers sexués, n'ayant pas les mêmes attentes des personnes qui s'y forment (Chapitre 6). En prolongement de cette question de la souffrance, seront ensuite examinées les stratégies adoptées par les jeunes pour y faire face. Ces dernières révèlent les spécificités de cette population apprentie et des contraintes particulières qui s'exercent à leur encontre. Certaines stratégies peuvent être qualifiées de stratégies de genre, elles illustrent ainsi qu'apprendre à se défendre fait partie de la socialisation professionnelle et que celle-ci est teintée par l'environnement professionnel sexué dans lequel elle se déroule (Chapitre 7). Enfin, des réflexions plus transversales sur la formation professionnelle comme fabrique du genre seront menées. Elles s'intéresseront à l'apprentissage de la division sexuelle du travail et des normes de genre. Les analyses s'arrêteront sur la façon dont ces apprentissages se font, sur la violence qui les sous-tend et sur ce qu'ils recouvrent. En effet, l'apprentissage des rapports sociaux de sexe et de leur antagonisme est doublé par celui du système hétéronormatif et de son discours sur la complémentarité entre les sexes (Chapitre 8). 
La formation professionnelle se révèle donc un laboratoire passionnant pour étudier les mécanismes de la socialisation de genre, au travers de la division sexuelle du travail, des normes de genre attachées à des métiers fortement sexués. Plus encore, elle permet d'appréhender les formes souvent brutales de cette socialisation: rappels à l'ordre, mises à l'épreuve, menace de déclassement. Preuve en est que le genre n'est à aucun moment une traduction sociale du sexe, mais qu'il est bien le résultat de divers processus de socialisations, intervenant dans des cadres nombreux et variés: de la famille à l'école, des loisirs au travail, et bien sûr en formation professionnelle. 


\section{Chapitre 1 \\ Les inégalités de sexe en formation professionnelle en Suisse}

Afin de souligner quelques-uns de ses principaux enjeux, un portrait général de la formation professionnelle est esquissé ici. Il s'agit de rappeler le cadre historique qui a vu se développer cette filière de formation, ainsi que son imbrication étroite avec le marché du travail. Ainsi, analyser ce qui se passe en formation professionnelle n'est pas sans rappeler les enjeux théoriques de la sociologie du travail féministe (ségrégation, phénomène de concentration, etc.). Ces phénomènes sont illustrés non seulement au travers d'un volet historique, qui met en évidence la constitution de la formation professionnelle comme un espace ségrégué, mais encore par l'analyse des changements récents ayant marquéla formation professionnelle. En effet, d'une part, on assiste à l'intellectualisation des filières, qui participe de la hiérarchisation des professions et du repli ${ }^{6}$ de certaines d'entre elles, particulièrement peu mixtes. Ces logiques de bastions créent de fait un processus d'exclusion des personnes atypiques. D'autre part, la situation sur le marché des places d'apprentissage (décalage entre offres et demandes de places) ${ }^{7}$ participe du phénomène d'allongement et de complexification de la transition entre le secondaire I et le secondaire II. Ces changements touchent en particulier les jeunes femmes, exposées aux processus de ségrégation horizontale et de concentration des emplois et subissant dès lors une «sur-sélection ${ }^{8}$ et une concurrence accrue.

6 Ce terme est utilisé dans le sens où dans les professions masculines menacées, on assiste à une forme de repli identitaire sur des valeurs viriles, qui ont longtemps participé de la construction de l'identité masculine au travail (Couppié et Epiphane, 2007).

7 La situation de décalage quantitatif et qualitatif sur le marché des places d'apprentissage a fortement marqué cette étude. La recherche ayant démarré en 2006, la situation sur le marché des places d'apprentissage était particulièrement tendue. La situation a évolué depuis et aujourd'hui, si certains secteurs restent difficiles d'accès, le marché s'est dans les grandes lignes détendu.

$8 \quad$ Le terme est de Moreau (2000) : les filles s'orientant dans un nombre moins important de professions, il y a donc moins de places d'apprentissage à disposition et une sélection accrue des candidates. Ce terme sera repris tout au long de cette analyse. 


\subsection{Regard rétrospectif sur la mise en place du système suisse de formation professionnelle}

Comprendre les enjeux actuels du système suisse de formation professionnelle nécessite de faire un détour historique par les principales étapes de sa mise en œuvre. De même, il est indispensable de revenir sur la construction historique $\mathrm{du}$ " retard des femmes en matière de formation " pour comprendre ce qui se joue aujourd'hui en termes d'inégalités dans le champ de l'éducation, en particulier en formation professionnelle.

\subsubsection{La formation professionnelle: une histoire écrite au masculin}

Historiquement, la formation professionnelle se développe dans les traces du salariat. Avec la révolution industrielle, le besoin d'une main d'œuvre qualifiée se fait sentir (Lamamra et Masdonati, 2009), et c'est ainsi dans le dernier tiers du XIX ${ }^{\mathrm{e}}$ siècle - avec la Loi sur les Fabriques de 1877 et la création, en 1879, de l'Union suisse des arts et métiers (USAM) - qu'une véritable formation professionnelle voit le jour (Collectif, 2007). Dans un premier temps, la main d'œuvre qualifiée est recrutée à l'extérieur des frontières. Mais la pénurie liée aux conflits mondiaux, ainsi que la nécessité de former des cadres, contraignent le patronat à développer une formation sur place (Waardenburg, 2007). S'ajoute à cela la peur de la concurrence étrangère, ainsi très vite la formation professionnelle devient un enjeu de politique nationale: «On est pénétré de la nécessité de faire tout ce qui sera possible en faveur de l'enseignement professionnel, afin de ne pas rester en arrière des progrès que le temps amène avec lui et de pouvoir soutenir la lutte contre la concurrence étrangère. Il n'y a aucun doute que le moyen le plus efficace de soulager les industries et les métiers dans le malaise, consiste dans l'amélioration de l'enseignement qui s'y rapporte» (Conseil Fédéral, 1883: 656-657)9 ${ }^{9}$. Il s'agit aussi d'éduquer la classe ouvrière, de contrôler les "classes dangereuses", car sans formation, notamment professionnelle «les apprentis et les ouvriers, malgré leur meilleure volonté de marcher en avant, resteront, leur vie durant, ce qu'ils ont été dans l'atelier. Aussi, aigris par l'inutilité de leurs efforts, ils seront relégués dans la grande armée de ceux qui se dressent contre l'état, qui ne les a pas instruits, et contre le capital, qui les écrase» (Commission du Conseil des Etats, 1884 : 942). Enfin, il s'agit encore d'adapter l'humain à l'économie, ce quel'Union syndicale suisse,

9 Les documents historiques ont été mis à ma disposition par mon collègue Lorenzo Bonoli (2011), que je remercie ici. 
qui préférerait le mouvement inverse, soit d'adapter l'économie aux besoins humains, dénonce (Bois, 1980). Cette volonté de formation tant morale que technique des masses laborieuses apparaît dès les premières lois régissant la formation professionnelle en Suisse : «...mais ce qui est en premier lieu indispensable, c'est non d'apprendre aux enfants tel ou tel métier, ni même les orienter vers une profession répondant à leurs aptitudes, mais de leur inculquer, le goût du travail, l'amour du devoir accompli» (ASCAPA, 1920:89-90). Après la Seconde Guerre mondiale, l'essor économique et la période de plein emploi vont asseoir la prépondérance du système dual et sa propension à modeler la formation des hommes d'abord. Il assure alors une qualification minimale des jeunes hommes de nationalité suisse. Ainsi au départ, en Suisse comme dans les pays voisins, «l'enseignement technique et professionnel est d'abord un univers masculin. Il n’a longtemps scolarisé que des garçons vers des métiers masculins, ne réservant aux filles qu'une place marginale [...] Cette inégalité de scolarisation ne fait que traduire les fortes résistances longtemps opposées à la professionnalisation des femmes » (Baudelot et Establet, 1992b: 178).

\subsubsection{Le "retard historique" des femmes en matière de formation}

La formation professionnelle étant réservée aux hommes, à l'instar des autres domaines du savoir, les femmes connaissent ici aussi un « retard historique». Lorsque se généralise le droit àl'instruction pour les femmes, elles sont concentrées dans les " écoles de jeunes filles » censées répondre «à la volonté de la classe moyenne ${ }^{10}$ soucieuse de la bonne éducation de sa progéniture d'occuper le plus judicieusement possible les jeunes filles entre la fin de la scolarité obligatoire et le mariage, en les formant à leur futur rôle de mère et de ménagère par l'acquisition de compétences dans les domaines domestique, éducatif et des soins» (Imdorf, 2004: 110). Ces lieux de formation sont également censés répondre aux besoins de la bourgeoisie industrielle qui - suite à la crise du dernier quart du XIX siècle - ne peut plus nécessairement proposer à ses filles comme seul horizon le modèle de la famille bourgeoise et doit leur fournir une ins-

10 La notion de «classe moyenne » est utilisée en Suisse à la fin du XIX ${ }^{\mathrm{e}}$ siècle par la bourgeoisie comme rempart dans la lutte contre la gauche et le mouvement ouvrier. Non seulement, elle doit servir à réduire les antagonismes sociaux (un échelon intermédiaire se plaçant entre la bourgeoisie et le prolétariat), mais devrait à terme devenir l'idéal d'émancipation des milieux ouvriers (Jost, 1992). 
truction leur permettant d'exercer une activité salariée jugée convenable (Dallera, 2003). De manière générale, l'éducation des filles, comme celle des garçons, était, et le reste à certains égards, largement conditionnée par leurs destins sociaux: l'éducation des enfants au sein de la famille pour les unes (Mayeur, 1991; Mosconi, 1994), l'éducation à la citoyenneté et l'élévation morale pour les autres (Chaponnière et Chaponnière, 2006). Dans cette perspective, «il n'allait pas de soi que leur scolarité [celle des jeunes femmes] les conduise à l'apprentissage d'un métier » (G. Moreau, 2000: 69); de son côté, «l'apprentissage professionnel avait à l'origine pour vocation de former les jeunes hommes à des professions qui puissent leur garantir un revenu. Le système dual n'était guère pensé par rapport aux femmes» (Imdorf, 2004: 110).

Parallèlement, et dès l'émergence du salariat, le patronat a cherché à disposer d'une main d'œuvre bon marché et flexible (Scott, 1991). C'est dans cette perspective que les femmes, professionnellement actives de longue date, notamment dans l'agriculture ou à domicile durant la phase de proto-industrialisation, ont été embauchées dans les usines. Pour justifier les bas salaires et la flexibilité, il était nécessaire qu'elles demeurent des travailleuses non qualifiées, dans l'industrie textile par exemple (à ce propos, voir Baudelot et Establet, 1992b). Perçues comme des concurrentes à la main d'œuvre masculine, non défendues par les syndicats (Scott, 1991) et voyant leurs tentatives de professionnalisation attaquées (Baudelot et Establet, 1992b), les femmes ont été non seulement tenues à l'écart du développement de la formation professionnelle, mais aussi maintenues dans le bas des positions professionnelles. La division sexuelle du travail a ainsi été à l'œuvre dès la mise en place de la formation professionnelle: travail productif reconnu pour les hommes, et travail reproductif - auquel est associé le refus de la professionnalisation - pour les femmes. Accédant au mieux à une formation spécifique destinée à accomplir leur rôle de mère et d'épouse, elles ont d'emblée été victimes de logiques d'exclusion et de ségrégation, des logiques qui perdurent aujourd'hui. Il en est de même en France, où «l'apprentissage en entreprise présente des caractéristiques en partie similaires [au compagnonnage], et notamment un développement préférentiellement masculin " (G. Moreau, 2000: 69). Il faudra attendre l'essor du plein emploi durant les Trente Glorieuses pour voir enfin la généralisation du système de formation professionnelle. Ce n'est donc que dans les années 60-70 qu'on assiste à "la généralisation plus tardive de l'apprentissage chez les jeunes femmes et les jeunes de nationalité étrangère» (Collectif, 2007). 
Un dernier élément a contribué au développement genré de la formation professionnelle : la répartition de la responsabilité en matière de formation entre cantons et Confédération. Ainsi, «in the division of tasks and responsibilities between the cantons and the Confederation, education and health are under the sway of the cantons. Vocational education in industry and crafts, however, was considered to be a part of the economy, and hence subject to federal legislation and support. Federal legislation plays a role in socially defining a 'real' occupation, setting common standards for the whole country and providing for nationally acknowledged certification" (Borkowsky, 2001). Or, il s'avère que les secteurs exclus de la législation - et de la reconnaissance - fédérale sont des secteurs d'activité majoritairement occupés par des femmes (santé, éducation, travail social). Ce n'est qu'en 2000, avec l'entrée en vigueur de la nouvelle Constitution fédérale que cette situation a changé : désormais l'entier de la formation professionnelle est placé sous l'égide de la Confédération. De la création de la formation professionnelle jusqu'à très récemment, seules les formations soumises à la Loi fédérale sur la formation professionnelle, toutes à dominante masculine, étaient reconnues au niveau national (Borkowsky, 2001). Ce faisant, une division s'est instaurée entre activités et professions, qui recoupe largement la partition entre métiers féminins et professions masculines.

Ce bref détour historique rappelle que la formation professionnelle s'est constituée au masculin et que la situation actuelle de ségrégation en est probablement l'héritage. En outre, cette-dernière s'articule aux différents phénomènes (ségrégations, concentration, discriminations) en vigueur sur le marché du travail.

\subsection{Un système de formation lié au marché de l'emploi}

Dans un contexte où près de $2 / 3$ des jeunes font leur formation post-obligatoire dans la voie professionnelle, une très nette majorité d'entre elles et eux $(80 \%)$ optent pour le système dual, qui alterne formation théorique en école et formation pratique en entreprise (SEFRI, 2015). Le système dual, issu de la même tradition que le modèle allemand, est ainsi étroitement lié au marché de l'emploi. D'une part, les lieux de formation sont des entreprises soumises aux impératifs de rentabilité et de productivité et, d'autre part, les personnes issues d'une telle formation doivent être immédiatement «employables» sur le marché du travail. Les mutations intervenues dans le marché del'emploi influencent donc très directement le système de formation professionnelle duale. En outre, on peut parler 
d'un marché des places d'apprentissage, qui fonctionne de près ou de loin sur le modèle du marché du travail. Les jeunes doivent donc se profiler sur ce marché pour trouver une place d'apprentissage. Elles et ils y sont soumis.e.s à des logiques de compétition, de concurrence, de ségrégation et de discriminations similaires à celles en vigueur sur le marché du travail (P. Kergoat, 2011, 2014). Ce lien étroit entre marché du travail et formation professionnelle est renforcé par l'organisation du système. En effet, la politique de formation est négociée entre trois partenaires: la Confédération, les cantons et les organisations du monde du travail (Dubs, 2006). Les organisations du monde du travail (associations professionnelles, associations patronales et syndicales) interviennent ainsi dans l'élaboration des plans de formation et dans la mise en place des cours interentreprises (SEFRI, 2015). Les enjeux du marché du travail ne sont donc jamais loin du système de formation professionnelle, $\mathrm{y}$ compris lors de l'élaboration des plans de formation et autres curricula.

\subsubsection{Inégalités sur le marché du travail et de la formation professionnelle}

Le lien entre marché du travail et formation professionnelle invite à faire un détour du côté des inégalités en vigueur aujourd'hui encore sur le marché du travail. Malgré la féminisation du salariat, la progression des scolarités féminines et l'homogénéisation des comportements d'activité masculin et féminin, les inégalités en matière de salaires et de carrières résistent tandis que les ségrégations et les discriminations se reconstruisent (Maruani, 2006). Ainsi, le paradoxe bien connu - des filles ayant un capital scolaire élevé, mais des difficultés à le négocier sur le marché du travail - apparaît de manière particulièrement aiguë (voir notamment Couppié et Epiphane, 2004). Cela est d'autant plus marqué dans le contexte helvétique, où les inégalités sur le marché du travail (écarts salariaux, ségrégation horizontale, taux d'activité, ségrégation verticale) sont singulièrement frappantes en regard à d'autres pays (OFS, 2008b). L'écart entre niveau de formation et position dans l'emploi révèle la complexité du lien entre formation et emploi. Cela se vérifie déjà au niveau de la formation professionnelle, puisque les filles quittent le secondaire avec un capital scolaire élevé et sont pourtant plus nombreuses à devoir attendre l'accès à une première place d'apprentissage (Meyer, 2004; Rastoldo, Evrard et Amos, 2007). Couppié et Epiphane (2004) font partie des chercheur.e-s qui bousculent l'idée communément répandue selon laquellel'insertion professionnelle ne serait que la simple traduction, dans le monde du travail, d'un parcours scolaire. Ils critiquent 
le lien quasi causal et automatique établi entre formation et emploi, lien qu'ils définissent ainsi : «ne suivant pas les mêmes formations, les jeunes femmes occuperaient logiquement des professions différentes. Les systèmes d'allocation des individus dans les différents groupes professionnels prolongeraient donc, mécaniquement, la ségrégation éducative induite par les orientations sexuellement clivées » (Couppié et Epiphane, 2004: 10). C'est sans compter sur les trajectoires différentes et la multiplicité des destins professionnels de personnes ayant un même capital scolaire. Selon l'analyse quantitative proposée par les auteurs, deux tiers de la ségrégation seraient imputables aux effets de l'orientation (sur les effets de la socialisation scolaire et les choix professionnels, voir Chapitre 3). Si l'on peut en partie faire le lien entre formation suivie au secondaire, orientation et formation professionnelle entreprise, d'autres paramètres interviennent, comme cela sera développé plus loin (voir Chapitre 3). Couppié et Epiphane (2004) signalent que la formation n'a qu'une part relative sur la position dans l'emploi et qu'à la ségrégation liée à l'orientation, s'ajoute celle en vigueur sur le marché du travail. Ainsi, et malgré les changements signalés précédemment, la « ségrégation demeure un des traits structurants du marché du travail » (Maruani, 2006:37). Le marché des places d'apprentissage étant étroitement lié au marché du travail, les principaux éléments de la ségrégation professionnelle interviennent déjà au niveau de la formation professionnelle : la concentration, la ségrégation horizontale, la division sexuelle du travail et les discriminations. Cependant, d'autres aspects, tels que la ségrégation verticale, les écarts salariaux et les différences de taux d'activité ne sont pas encore présents de manière récurrente dans la population apprentie.

\section{Concentration et sur-sélection des filles}

«La non-mixité a un premier indicateur simple: la concentration des emplois féminins. Hommes et femmes ne se répartissent pas de manière égale dans les différents secteurs de l'économie. La majorité des emplois féminins reste concentrée dans un petit nombre de métiers et secteurs d'activité » (Maruani, 2006: 37) et ce avec une constance surprenante, puisque «les six professions les plus féminisées en 2005 sont les mêmes que celles qui l'étaient en $1962 »$ (Maruani, 2006: 37). Si l'on s'intéresse aux 12 professions les plus choisies par les jeunes au sortir du secondaire, le phénomène de concentration apparaît très nettement: pour réunir la moitié des jeunes femmes $(52,8 \%)$, quatre professions suffisent, alors qu'avec les 12 professions les plus choisies, on ne parvient même pas à réunir la moitié des jeunes hommes (47,3\%) (pour le détail, voir Annexe 2). 
Ce phénomène de concentration prend une teinte particulière dans le contexte de la formation professionnelle duale. En effet, dans un contexte de décalages entre offre et demande de places d'apprentissage, la structuration du marché du travail ou du marché des places d'apprentissage (ségrégation, concentration) crée une plus grande concurrence entre les femmes et ce, dès l'entrée en formation professionnelle (Imdorf, 2004). En effet, les filles, relativement aux garçons qui se répartissent dans un plus grand nombre de professions, sont plus nombreuses pour moins de professions. Dès lors, elles subissent une plus forte sélection et leur probabilité d'entrer immédiatement en formation professionnelle au sortir du secondaire est plus faible. Comme déjà évoqué précédemment, Moreau qualifie ce phénomène de «sur-sélection» (G. Moreau, 2000).

\section{Ségrégations horizontales et verticales}

Au phénomène de concentration est directement associéle fait que femmes et hommes ne s'insèrent pas dans les mêmes secteurs. Au sein des 12 professions les plus choisies par les jeunes, seules trois sont communes aux filles et aux garçons (employé.e de commerce; gestionnaire de commerce de détail; cuisinière et cuisinier). La sexuation des professions et des filières de formation est très marquée. Cette inégale répartition dans les secteurs, cette connotation des emplois comme étant «féminins» ou "masculins», est nommée par les sociologues du travail «ségrégation horizontale ». Elle se répercute en formation professionnelle sur les filières de formation, elles aussi fortement ségréguées (OFS, 2013).

La sexuation des filières de formation intervient à tous les niveaux, que ce soit en formation professionnelle initiale ou dans les hautes écoles spécialisées et universitaires (OFS, 2008b). De toute évidence, filles et garçons ne se dirigent pas ou ne sont pas dirigé.e.s dans les mêmes secteurs d'activité. Ce phénomène perpétue la ségrégation horizontale en vigueur sur le marché du travail. Il se traduit également par un nombre très restreint de filières ou de professions mixtes. D'ailleurs, lorsqu'une relative mixité existe, une analyse plus fine montre que les emplois occupés (par exemple employé.e.s de commerce) ne sont pas identiques et que "hiérarchies et clivages se recréent au sein de professions devenues mixtes» (Maruani, 2006: 40).

Parallèlement à la ségrégation horizontale se perpétue la ségrégation verticale. Malgré les changements intervenus sur le marché du travail, les possibilités d'accès à des postes élevés dans la hiérarchie demeurent rares pour la plupart des femmes. Elles sont concentrées dans les emplois d'exécution et occupent des postes peu ou pas qualifiés. «Il 
semble en effet que < quelque chose > résiste dans l'univers des emplois a priori mixtes, qu'il reste difficile pour des femmes dont les atouts sont importants (au prorata des diplômes et de la qualification) d'occuper les mêmes positions que les hommes» (Cacouault, 1995, citée par Maruani, 2006 : 40). Cependant, un certain nombre de femmes ont pu, grâce à leur capital scolaire notamment, accéder à des professions qualifiées et à des postes supérieurs. Dès lors, un double mouvement s'opère: «il y a à la fois plus de femmes qualifiées, accédant à des fonctions et professions traditionnellement masculines, et plus de femmes occupant des postes peu ou pas qualifiés et, pour la plupart, dans des secteurs très féminisés. La coexistence de ces deux mouvements débouche beaucoup plus sur le constat d'une bipolarisation que sur celui d'une ségrégation immobile» (Maruani, 2006: 41-42).

La question de la ségrégation verticale, et les travaux cherchant à en expliquer les mécanismes (plafond de verre, etc.), ne seront pas retenus ici, puisque la formation professionnelle se situe en amont, à un moment où apprenti.e. $\mathbf{s}^{11}$, filles et garçons, occupent encore des statuts subalternes, tout en bas de la hiérarchie des entreprises. Pour autant, la hiérarchie qui s'instaure entre apprenti.e.s (voir notamment Chapitre 8) pourrait laisser penser qu'une forme de ségrégation verticale existe (elle serait organisée sur la base de l'ancienneté).

\section{Division sexuelle du travail}

La division sexuelle du travail (Kergoat, 2000) se traduit sur le marché du travail par une séparation et une hiérarchisation des activités selon qu'elles sont attribuées aux femmes ou aux hommes. Ainsi, outre l'assignation de certaines tâches (dans la sphère professionnelle ou domestique) aux personnes en fonction de leur position réciproque dans les rapports sociaux (notamment de sexe) et la connotation de ces tâches selon une échelle «masculin/valorisé »- «féminin/dévalorisé», la division sexuelle

11 Le terme «apprenti-e» est utilisé pour parler des jeunes en formation professionnelle. Cet usage a été uniformisé, bien qu'au début de la recherche (2006), il était attendu de parler d'«apprenant.e», pour respecter la nomenclature imposée par la Nouvelle Loi sur la formation professionnelle (NLFPr, 2004). Le terme d'apprenant.e n'a guère été utilisé, car les personnes concernées ne l'adoptaient pas pour parler d'elles-mêmes. C'est pourquoi il ne figure pas dans le canevas d'entretien. En outre, à la suite des nombreuses résistances rencontrées par ce changement, l'Office fédéral de la formation et de la technologie (OFFT, aujourd'hui SEFRI), dont dépend la formation professionnelle suisse, accepte aujourd'hui l'utilisation conjointe des deux termes (apprenti.e et apprenant.e). 
du travail se reconfigure partout où l'avancée en mixité aurait pu laisser penser qu'elle allait s'éteindre d'elle-même. Dans les professions mixtes, femmes et hommes ne font pas les mêmes tâches, ne sont pas assigné.e.s aux mêmes activités. Gasparini (2007) donne à ce propos un intéressant exemple dans les postes de vente liés au sport. On assiste dans ce secteur à une nouvelle division sexuelle du travail: «Le textile aux femmes, le matériel aux hommes» (Gasparini, 2007: 142). Ce processus estégalement observé dans les insertions non traditionnelles, où la division sexuelle du travail se reconfigure. Ainsi, dans des postes connotés comme féminins, tels que l'accueil pour une compagnie de transport public (Lada, 2008), les agent.e.s, de même que le public, opèrent une division des tâches. Le port de charges lourdes, la commande de fournitures ainsi que les questions techniques et d'orientation reviennent aux hommes. Le phénomène inverse se retrouve chez les femmes insérées dans des professions traditionnellement masculines, qui se voient assignées à des tâches d'entretien ${ }^{12}$, confinées à des " postes de femmes » (Eckert et Sulzer, 2007), tout comme leur est réservée la part plus «féminine » de l'activité, à l'exemple des femmes chauffeurs routiers qui sont plus fréquemment assignées au transport de personnes sur de courtes distances. Parfois, ce sont les femmes elles-mêmes qui s'identifient à leur activité en mettant en avant des "spécificités féminines», à l'instar des mécaniciennes se qualifiant «d'infirmières de l'automobile» (Marry, 2007).

Enfin, en Suisse, la prise en charge des tâches domestiques ne s'est guère modifiée et reste largement dévolue aux femmes, leurs conjoints ne prenant toujours pas leur part du travail d'entretien (OFS, 2008b). Cependant, plusieurs changements majeurs sont intervenus: les femmes des pays industrialisés ont peu à peu accédé à des postes élevés et relativement bien rémunérés, et elles ont délégué ces tâches à d'autres. En effet, la mondialisation deséchanges a engendré une bipolarisation des emplois féminins (Maruani, 2006) et contribué à modifier la division sexuelle du travail, qui est désormais étroitement liée à la division internationale du travail. Les femmes du Nord ayant la possibilité désormais de déléguer le travail domestique (entretien, élevage des enfants, etc.) à des femmes récemment arrivées dans les pays occidentaux ou occupant une position sociale moins favorable (Dallera, 2007; Ibos, 2008).

12 Le terme entretien est entendu ici comme synonyme de ménage. Les termes tâches domestiques, tâches de ménage ou tâches d'entretien seront utilisés indifféremment. Contrairement à Delphy (1998) dont l'usage renvoie explicitement aux échanges au sein d'un couple, les femmes effectuant le travail domestique contre leur «entretien" par leur époux. 


\section{Les discriminations}

Tous ces éléments ont des incidences sur la réalité professionnelle des femmes et se traduisent par un certain nombre d'inégalités et de discriminations, sur lesquelles cette section ne s'attardera pas. Deux tendances doivent cependant être soulignées en ce qu'elles illustrent le spectre des discriminations que les rapports de travail font vivre aux femmes. D'une part, l'insertion dans l'emploi à temps partiel, souvent imposé, a des conséquences en termes de mobilité de carrière, d'accès à la formation continue, de salaires et de répartition du travail domestique. D’autre part, bien des femmes sont confrontées à des comportements sexistes, allant de la plaisanterie au harcèlement sexuel, en passant par les environnements de travail hostiles (affiches de femmes dénudées, voire pornographiques). Ces comportements s'expriment en force dans les bastions masculins, qui cherchent à s'affirmer contre l'entrée des femmes dans leur secteur, mais ne se limitent pas à ces lieux. Ces discriminations signifient aux femmes que, malgré les avancées, le monde du travail ne leur est pas encore acquis et constitue autant de mises à l'épreuve.

Pour la population apprentie, certaines de ces discriminations ne sont pas encore d'actualité (temps partiel imposé, accès à la formation continue, etc.). En revanche, certaines pionnières font déjà l'expérience du sexisme et des diverses formes de résistance masculine à leur présence, pendant que d'autres apprenties (en situation d'insertion dans des secteurs mixtes) découvrent les inégalités salariales en comparaison à leurs collègues apprentis ${ }^{13}$.

\subsubsection{Impacts des changements récents du marché du travail sur la formation professionnelle}

L'interdépendance de la formation professionnelle avec le marché de l'emploi fait que l'apprentissage est assez sensible à la conjoncture et aux changements qui interviennent sur le marché du travail (Hanhart, 2006).

Ces dernières décennies, le monde du travail a connu de profondes transformations (Castel, 1995), notamment par la tertiarisation de l'emploi, l'innovation technologique et la disparition de certains métiers traditionnels, remplacés par de nouvelles professions issues

13 La question des écarts salariaux entre apprenti.e.s n’a pas été étudié de manière systématique pour l'instant. En effet, le tissu économique de Suisse romande étant composé principalement de PME, il n'est pas rare que les entreprises

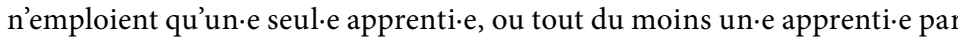
année de formation professionnelle. Une analyse comparative des salaires serait de ce fait relativement délicate à mener. 
du regroupement de professions existantes et dont les formations sont souvent plus exigeantes et plus longues ${ }^{14}$. Ces mutations transforment le marché du travail, qui requiert plus que jamais des personnes polyvalentes et flexibles, pouvant s'adapter aux évolutions parfois rapides des professions, tout en étant spécialisées et fiables dans leur champ de compétences (Poglia, 1999).

Pour répondre à ces exigences, la formation professionnelle suisse a entamé une profonde transformation au cours des quinze dernières années. Le niveau général de la formation a augmenté et s'est «intellectualisé» (U. Moser, 2004; Poglia, 1999). Ce changement se reflète dans la nouvelle Loi sur la formation professionnelle (NLFPr), entrée en vigueur le $1^{\text {er }}$ janvier 2004 : bien qu'elle mette en place des passerelles («maturité professionnelle») pour accéder aux formations supérieures, elle élève dans le même temps le niveau d'exigences des filières destinées aux jeunes en difficulté, à l'instar de la «formation professionnelle initiale en deux ans $»^{15}$ (pour une vue d'ensemble des possibilités offertes par le système de formation professionnelle, voir SEFRI, 2015). Si ces réformes préparent les futur.e.s travailleurs et travailleuses à relever les nouveaux défis du monde professionnel, elles risquent donc aussi d'exclure un nombre croissant de jeunes de cet univers (Meyer, 2003; U. Moser, 2004).

\section{Un contexte qui fragilise les futur.e.s apprenti.e.s}

Avec l'élévation du niveau général d'exigences et l'intellectualisation de la formation professionnelle (U. Moser, 2004; Poglia, 1999), on peut considérer qu'il existe à terme un risque d'exclusion des personnes dotées du capital scolaire le plus faible. Cette logique est renforcée par les procédures de sélection utilisées par les PME et par les grandes entreprises,

14 Le processus est en cours et les associations professionnelles travaillent actuellement sur les cadres législatifs (ordonnances de formation) accompagnant ces nouvelles professions. A titre d'exemple, la formation plus exigeante de polymécanicien regroupe et remplace huit anciennes professions, et la durée des formations dans la vente et les services (emplois de bureau) est passée de deux à trois ans.

15 La formation professionnelle initiale en deux ans remplace l'ancienne formation élémentaire, qui était basée sur la mise en place d'objectifs de formation individualisés. Si les deux modèles sont destinés aux jeunes en difficulté, la nouvelle formation implique des exigences plus élevées que la précédente, notamment parce qu'elle introduit des objectifs de formation uniformisés - et donc non adaptés aux besoins et possibilités de chaque apprenti.e. La formation professionnelle initiale en deux ans a cependant l'avantage de fournir aux apprenti.e.s un encadrement individuel spécialisé et des appuis pédagogiques censés les aider à atteindre ces objectifs. 
qui multiplient les tests, notamment en ce qui concerne les aptitudes scolaires. A ces examens «maisons » sur lesquels les personnes chargées de la sélection s'appuient fortement s'ajoutent des méthodes externes de sélection standardisées (Basic- et Multicheck) (Imdorf, 2007b).

Simultanément, le décalage entre le nombre dejeunes qui cherchent un apprentissage et le nombre de places vacantes persiste (Hanhart, 2006) ${ }^{16}$. Cet écart est tant quantitatif que qualitatif, puisque l'offre de places d'apprentissage, déjà peu nombreuses, ne correspond pas forcément aux désirs des jeunes (LINK, 2005 ; Rastoldo, 2006). Ainsi, nombre de jeunes sont soit dans l'obligation d'apprendre un métier non choisi (choix par défaut), soit dans l'impossibilité de se former.

Un choix par défaut constitue un facteur de risque et augmente les probabilités d'un décrochage ultérieur (voir Chapitre 2) (Rastoldo et al., 2009; Schmid, 2010). En d'autres termes, même si les jeunes qui ont opté pour un métier «faute de mieux» ont pu, dans un premier temps, mieux négocier la transition école-formation professionnelle que les jeunes qui n'ont pas trouvé d'entreprises formatrices, il se peut que cette solution ne soit pour eux et elles aussi que "transitoire» (Meyer, 2004).

Le contexte général de la précarité du travail et de la formation pèse sur ces jeunes, et notamment le risque de chômage et de rupture de contrat d'apprentissage. Le taux élevé d'interruptions d'apprentissage dual (Stalder et Schmid, 2006a) ${ }^{17}$ peut être vu comme un signe tangible de cette pression ${ }^{18}$, touchant autant les filles que les garçons (Stalder et Schmid, 2006a). Les risques d'arrêt prématuré en formation professionnelle dépendent en outre d'autres facteurs sociaux, qui deviennent particulièrement influents lorsqu'ils se cumulent (voir Chapitre 2).

16 L'Union syndicale suisse estime par exemple qu'il manque aujourd'hui 10 '000 places d'apprentissage (http://www.sgb.ch/schwaab-blog/index.php).

17 Même si aucune statistique n'existe au niveau national, des données cantonales sont parfois disponibles. De manière très générale, on observe des taux de rupture d'apprentissage allant de 10 à $30 \%$ selon les régions et la méthodologie utilisée. Malgréles différences cantonales, ces taux sont stables depuis une dizaine d'années. A titre d'exemples, les taux de résiliation de contrats d'apprentissage vont de 12\% (canton de Neuchâtel 2004/2005), 13\% (canton de Vaud 2001/2002), à 24\% (canton de Berne 2004/2005), voire 29\% (canton de Genève 2000/2001) (Stalder et Schmid, 2006a, 2006b).

18 La pression liée au décalage entre offre et demande de places d'apprentissage, fait que les jeunes choisissent plus fréquemment un métier "par défaut». Ce choix «faute de mieux» a des implications sur le déroulement de la formation. En effet, dès lors que des problèmes sont rencontrés, ce choix par défaut agit comme un facteur aggravant. Ainsi, le contexte a-t-il également des répercussions sur les personnes qui ont trouvé une solution. 
Des secteurs masculinisés qui se replient et fonctionnent dans une logique de bastion

L'intellectualisation générale des filières de formation professionnelle signifie indirectement une forme de déclassement pour les voies les plus manuelles. Elle s'inscrit dans un mouvement en cours depuis plusieurs décennies, notamment avec les changements technologiques, qui provoque la dévalorisation de certains métiers traditionnels del'industrie, de l'automobile ou du bâtiment - majoritairement occupés par des hommes (Maruani et Nicole, 1989; Molinier, 2000). A cette dévalorisation objective, à l'heure de la tertiarisation de la formation professionnelle, s'ajoute un sentiment de déqualification vécu par les ouvriers et les techniciens face aux changements du travail, mais aussi face au chômage (Marry, 2006). Dès lors, les travailleurs cherchent à se protéger contre l'entrée des femmes, les secteurs se rigidifient et fonctionnent comme de véritables bastions (Couppié et Epiphane, 2007; Marry, 2006; Molinier, 2000): «En défendant leurs postes contre la féminisation, c'est leur idée la plus profonde d'eux-mêmes en tant qu'hommes que les hommes entendent protéger, surtout dans le cas de catégories sociales comme les travailleurs manuels [...] qui doivent une grande partie, sinon la totalité, de leur valeur, même à leurs propres yeux, à leur image de virilité » (Bourdieu, 1998 : 103). Cette résistance prend la forme de réflexes virilistes (Eckert et Sulzer, 2007; Guichard-Claudic et al., 2008; Molinier, 2000), d'attitudes sexistes (Lada, 2008; Laufer, 2009; Marry, 2008), d'intimidation et de violence (Legault, 2001; Marry, 2008), voire de mises à l'épreuve (Eckert, 2007; Lemarchant, 2007; Molinier, 2000). Ces attitudes de rejet peuvent être d'autant plus vives que les hommes se perçoivent comme des « communautés » fortement construites sur une opposition de genre (Pfefferkorn, 2008: 117). On peut donc faire l'hypothèse que certains secteurs, dans lesquelsl'identité professionnelle est largement construite sur la virilité, fonctionnent particulièrement sur le mode du rejet. Pour le groupe des hommes, l'enjeu est de taille, car la seule présence des femmes met en cause les valeurs sur lesquelles l'identité masculine s'est traditionnellement construite : à travers et autour du travail (Couppié et Epiphane, 2007). Ils peuvent donc percevoir l'entrée des femmes dans leurs bastions comme une menace qui enlèverait à ces métiers leurs dernières lettres de noblesse. Ce risque est d'autant plus élevé que les professions sont socialement peu valorisées et que le travail est pénible physiquement. Auparavant, à la dureté de la tâche étaient associées des qualités viriles, valorisantes pour les ouvriers par exemple. Mais dès lors que les nouveaux modes de production se mettent en place, qu'une 
femme peut aussi bien qu'un homme réaliser tel ou tel travail, le faire ne demande plus aucune spécificité virile (Molinier, 2000).

\section{Des logiques de ségrégation accrues pour les femmes}

Un autre paradoxe qu'il s'agit de relever ici c'est que l'intellectualisation des filières de la formation professionnelle ne s'accompagne pas de leur féminisation. On aurait pourtant pu penser que les jeunes femmes seraient favorisées, compte tenu de leur parcours scolaire au secondaire. Or il n'en est rien, cette supériorité en termes de qualifications au sortir du secondaire ne se traduit pas sur le marché des places d'apprentissage (Imdorf, 2004). Malheureusement, et bien que les logiques de sélection, en particulier dans les PME, soient fortement basées sur les qualifications scolaires (Imdorf, 2007b), les procédures intègrent également la division sexuelle du travail et la ségrégation professionnelle en vigueur. Dès lors, les femmes ne profitent pas de leurs compétences scolaires (Couppié et Epiphane, 2004). Le paradoxe souvent décrit d'une importante réussite scolaire des filles et de leur difficile insertion sur le marché du travail (Baudelot et Establet, 1992c; Baudoux et Noircent, 1997; Duru-Bellat, 1995c; Mosconi, 1994) apparaît très clairement ici.

\subsection{La transition école-travail : un enjeu central de la formation professionnelle}

Le système dual est reconnu en ce qu'il offre aux jeunes un espace institutionnalisé de transition entre deux mondes fort différents: celui de l'école obligatoire et celui du travail. Le caractère hybride de la formation duale peut permettre aux apprenti.e.s d'entrer en contact de manière progressive avec le monde du travail et de quitter graduellement leur identité d'élève. Ainsi, comme le souligne Cohen-Scali, l'alternance constitue une "transition aménagée » de l'école au travail (Cohen-Scali, 2001: 199). Depuis quelques années, cette entrée progressive vers le marché du travail est discutée, notamment en lien avec les travaux qui attestent de l'allongement et de la complexification du processus de transition (Duc et Lamamra, 2014; Hupka-Brunner, Grunder, Bergman et Imdorf, 2015; Lamamra et Duc, 2014).

\subsubsection{Un passage en douceur vers le monde adulte?}

L'attrait du passage vers l'âge adulte, symbolisé notamment par l'entrée sur le marché du travail, est évoqué fréquemment par les jeunes lorsqu'ils ou elles expliquent leur choix d'une formation professionnelle 
duale. L’entrée sur le marché du travail ne signifie donc pas uniquement l'accès à un salaire: elle indique surtout que les apprenti.e.s font partie du groupe des personnes actives.

Pourtant, la transition entre l'école obligatoire et la formation professionnelle duale implique pour les jeunes en fin de scolarité de trouver une place de formation en entreprise, idéalement dans la profession choisie. Mais les décalages qualitatifs et quantitatifs entre les places d'apprentissage offertes par les entreprises et la demande des jeunes créent une concurrence féroce pour obtenir une place de formation post-obligatoire. Cette dynamique désavantage les plus démuni.e.s, les obligeant à demeurer une ou plusieurs années dans des solutions transitoires $\left(10^{\mathrm{e}}\right.$ année de formation, mesures dans le cadre del'assurance-chômage, etc.) (Meyer, 2004). Cela concerne surtout les personnes d'origine étrangère, à bas statut socio-économique et à faible niveau scolaire (Imdorf, 2007b; Meyer, 2003; Rastoldo et al., 2007). Le passage par des solutions transitoires concerne également les filles (Meyer, 2003; Rastoldo et al., 2007). Dès la toute première confrontation au marché des places d'apprentissage, elles font l'expérience d'une forte sélection (G. Moreau, 2000) et d'une discrimination (Imdorf, 2004) qui risque d'affaiblir leur sentiment de légitimité à investir le monde du travail. Au final, il apparaît que le marché de l'emploi ne s'appuie pas seulement sur le capital de formation des individus, mais aussi sur des critères de structuration sociale tels que le genre, l'origine et le milieu social.

L’apprentissage dual est censé constituer un espace fondamental de transition entre l'école et le travail. Or, il se déroule en contact direct avec le monde du travail et ses contraintes. Les apprenti.e.s doivent donc gérer l'adaptation à un rythme soutenu, exigeant sur le plan physique et intellectuel, puisqu'il s'agit de cumuler les cours et l'immersion dans un environnement orienté vers la productivité. Le contact avec le monde du travail, même s'il est souhaité, peut ainsi s'avérer rude pour des personnes encore très jeunes (rappelons que l'entrée en apprentissage peut se faire dès l'âge de 15 ans). En apprentissage, les jeunes sont ainsi rapidement confronté.e.s à une ambivalence fondatrice du système dual. Cette ambivalence tient au statut même d'apprenti.e, qui doit composer avec deux aspects contradictoires: produire et apprendre (G. Moreau, 2000). Compte tenu du déroulement de la transition et des différents enjeux marquant l'entrée sur le marché de la formation professionnelle, la façon d'investir ces deux pôles peut différer selon le sexe: d'un côté, comme l'a montré Moreau (2000), des filles qui se sentent moins légitimes sur le marché du travail (par la sur-sélection et les discriminations qu'elles 
subissent) et qui investissent de ce fait davantage le volet formation; de l'autre, des garçons qui investissent davantage le volet emploi, car ils se sentent valorisés sur le marché du travail (notamment par la revalorisation qu'offre la formation professionnelle aux personnes ayant un passé scolaire difficile, voir à ce sujet Palheta, 2015 ou Renard, 2015).

\subsubsection{Difficultés accrues pour les filles lors de la transition}

Le phénomène de concentration des femmes dans un nombre restreint de professions (Imdorf, 2004; Jobin, 1995; OFS, 2008b) (voir Annexe 2) a un impact direct sur l'écart entre offre et demande de places d'apprentissage. Selon Imdorf (2004) et le baromètre de ces places (Lada, 2008 ; LINK, 2005; SEFRI, 2015), les branches qui attirent spécialement les jeunes femmes (notamment bureau, vente) enregistrent le plus grand nombre de candidatures. Cela s'observe également en France, générant une "sur-sélection des filles et à contrario une sous-sélection des garçons» (G. Moreau, 2000 : 73-74). Ainsi, les jeunes femmes ont-elles un risque accru de ne pas trouver de places d'apprentissage au sortir du secondaire, notamment dans leur domaine de prédilection.

Il en résulte que les jeunes femmes connaissent une transition plus chaotique et restent plus longtemps dans des solutions de transition $\left(10^{\mathrm{e}}\right.$ année, SEMO, etc.) (Imdorf, 2007b ; Meyer, 2004; Rastoldo et al., 2007; Stalder et Schmid, 2006a). Leur passage entre l'école et la formation professionnelle est plus difficile, alors même qu'elles présentent les meilleurs atouts en termes de qualifications scolaires. Autrement dit, bien que les employeurs insistent sur l'importance d'un bon niveau de scolarité dans la sélection d'un·e candidat.e (Imdorf, 2007b), la ségrégation en vigueur sur le marché du travail pèse déjà sur la teneur de la première transition.

Face à l'intellectualisation de la formation professionnelle et dans le contexte de rigidification et de résistance à l'entrée des femmes décrit précédemment, les difficultés augmentent pour les jeunes femmes qui souhaitent investir des domaines professionnels pionniers. Le terme pionnier étant pris ici dans le sens de non conforme à leur catégorie de sexe et donc à la place qui leur est normativement dévolue dans le champ professionnel. En effet, il n'est pas simple « de trouver sa place dansl'atelier lorsque les nouvelles venues y sont confrontées à l'hostilité sourde des hommes. Or, c'est bien de l'hostilité qu'elles rencontrent sur la chaîne de montage automobile, un univers de travail qui constituait, sinon une forteresse, du moins un bastion masculin» (Eckert, 2007 : 214). Il faut donc encore parler de pionnières, car comme le signale Marry (2007), on peut dire que dans certains secteurs (notamment les professions ouvrières), 
les pionnières se succèdent indéfiniment. La situation est d'autant plus paradoxale que, sur le plan politique, les injonctions à entrer dans des métiers traditionnellement investis par des personnes del'autre sexe sont fortes ${ }^{19}$. Mais ces démarches semblent davantage répondre aux besoins de main d'œuvre dans les secteurs délaissés (décalage offre - demande) qu'à une réelle volonté de mixité: l'entrée des femmes dans certains domaines «est directement liée aussi à certains facteurs structurels, notamment les décisions des pouvoirs publics de mettre fin aux discriminations vis-à-vis des femmes dans toute une série de professions qui leur étaient interdites, [aux] transformations qui ont marqué certains métiers dans une période récente (par exemple dans l'imprimerie ou le transport routier) ou encore [aux] difficultés de recrutement dans certaines professions. En effet, quand les "vocations" masculines se raréfient, quand les rémunérations sont faibles, quand le turn-over est important, organisations professionnelles et pouvoirs publics n'hésitent pas à se tourner vers les femmes et à promouvoir auprès d'elles des métiers qui leur étaient jusqu’alors fermés...» (Pfefferkorn, 2008: 113).

Ces politiques posent plusieurs problèmes qui ne seront qu'évoqués ici : premièrement, une logique sous-jacente de « rattrapage », les femmes devant "nécessairement» entrer dans les professions et les carrières masculines (Guichard-Claudic et al., 2008); deuxièmement, une logique individualisante, les pionnières devant démontrer leur motivation et leurs compétences à démarrer un parcours atypique (Pfister Giauque et Flamigni, 2010); troisièmement un discours incitatif, prônant que c'est possible, qu'il suffit de vouloir ${ }^{20}$, mais ne préparant pas aux difficultés que rencontreront vraisemblablement les pionnières. De fait, les deux dernières logiques font abstraction de la ségrégation du marché du travail et des résistances à l'œuvre dans certaines professions masculines.

Enfin, le décalage entre offre et demande de places d'apprentissages ne favorise en rien l'entrée dans une formation atypique. A côté des procédures de sélection "objectives», nombre d'autres aspects entrent en ligne de compte, notamment une forme de "paix sociale». Le choix d'une entreprise repose donc également sur la volonté ou le souci de

19 Depuis la fin des années 90, différentes campagnes de promotion des métiers atypiques auprès des filles ont été menées au niveau fédéral et cantonal, ou dans des entreprises et des lieux de formation: "Projet 16+», Campagne «Tekna», «Journée des filles», ou, dans le canton de Vaud, «Journée Oser tous les métiers».

20 Sur le mode de «Allez, les filles!» ou «C'est technique, c'est pour elles » ou encore "Osez la carrière!» 
recruter une personne qui ne mettra pas en péril cet équilibre, ou pour le dire comme un dentiste cité par Imdorf: «On écarte simplement du chemin tout ce qui pourrait poser des problèmes» (Imdorf, 2007b : 4). Si Imdorf rapporte surtout ce phénomène en ce qui concerne la discrimination à l'égard des jeunes d'origine étrangère, les pionnières se heurtent aux mêmes logiques, car elles pourraient venir perturber l'équilibre du collectif de travail ou le «confort» et les habitudes de la clientèle.

\subsection{La formation professionnelle, un espace avec ses logiques propres}

Si la formation professionnelle est largement tributaire de ce qui se passe sur le marché du travail, elle a cependant ses propres logiques. $\mathrm{Au}$ carrefour de la scolarité et de l'emploi, cet espace a ses enjeux et son fonctionnement spécifiques (Kergoat, 2015).

\subsubsection{Une formation qui passe par un modèle professionnel}

La première spécificité du système dual est une formation qui se déroule largement en entreprise et qui est encadrée par une personne formatrice (anciennement maître esse d'apprentissage). Lors de la formation pratique en entreprise, les jeunes sont constamment en contact avec une personne formatrice qui les initie au métier choisi. Or cette personne ne joue pas un rôle strictement formateur, elle est également un.e professionnel.le de référence, qui peut enseigner les «trucs du métier» (Filliettaz, De Saint Georges et Duc, 2008) et permettre l'accès au collectif de travail. Il s'agit alors d'une personne qui permet d'entrer dans le monde adulte, favorise l'intégration et l'acceptation de l'apprenti.e au sein de l'entreprise, et contribue par ce biais à sa socialisation professionnelle (Masdonati et Lamamra, 2009).

Il apparaît fréquemment que les apprenti.e.s attendent de cette personne qu'elle aille au-delà d'une stricte relation employeur-e-employé.e (Masdonati et Lamamra, 2009). Ainsi, les personnes formatrices sont souvent perçu.e.s comme des professionnel-le.s et des adultes de référence, et donc des personnes desquelles sont attendues proximité amicale et conseils.

Les responsables de formation en entreprise ne mesurent pas toujours la portée de leur rôle (Bahl, 2012; Capdevielle-Mougnibas, Cohen-Scali, Courtinat-Camps, de Léonardis, Favreau, Fourchard et Huet-Gueye, 2013), sans doute en raison d'une formation très condensée et des limites du soutien qu'elles et ils peuvent recevoir (notamment sous 
forme de coaching ou de supervision). En outre, nombre de personnes formatrices n'ont aucune décharge pour former, elles s'occupent des apprenti.e.s parallèlement à leur travail quotidien dans l'entreprise. A ce titre, elles sont, elles-aussi, prises dans la tension entre produire et former (Baumeler, Lamamra et Schweri, 2014).

Par ailleurs, la personne formatrice peut servir de modèle de genre. Compte tenu de la forte ségrégation en vigueur en formation professionnelle (non mixité de la plupart des filières), l'identité professionnelle se développe en étroit lien avec une identité de genre construite autour d'une unité sexe-métier (Baudelot et Establet, 1992b). Dans ce processus, on peut supposer que la personne formatrice joue un rôle non négligeable. Outre les différences d'encadrement interindividuelles constatées plus haut, la personne formatrice en entreprise est également un homme ou une femme de référence dans le contexte où évoluent les apprenti.e.s. Que ce soit volontairement ou non, l'appartenance sexuelle de la personne formatrice et son ancrage de genre lui servent d'expérience à partir de laquelle signifier à l'apprenti.e ce qu'implique être une femme professionnelle dans tel métier et être un homme professionnel dans tel autre. Le message ainsi donné par la personne formatrice peut cependant s'avérer problématique lorsque les attendus en matière de rôles sociaux de sexe sont trop contraignants ou que l'apprenti.e ne partage pas les normes de genre en vigueur dans l'entreprise, ou plus généralement dans la société.

La formation professionnelle duale se distingue notamment des autres filières de formation parce que la transmission des savoirs se fait dans différents lieux (école professionnelle, entreprise, cours interentreprises) ${ }^{21}$. La personne formatrice n'est donc pas la seule à rappeler à l'apprenti.e quels sont les attendus dans la profession, d'autres comme les collègues ou parfois la clientèle sont aussi des relais en ce sens. $\mathrm{Au}$-delà des compétences professionnelles, et parfois y compris pour ces compétences, les attendus sociaux sont clairement distribués selon une

21 Bien que l'on continue à parler de système dual (école professionnelle - entreprise), la nouvelle Loi fédérale sur la formation professionnelle a introduit un troisième acteur: les cours interentreprises, ce qui conduit certain.e.s à parler maintenant de système «trial» (SEFRI, 2015). Financés par les entreprises et mis en œuvre par les cantons ou les associations professionnelles, ces cours assurent des bases communes aux apprenti.e.s d'une même branche. Ce système permet aux entreprises de s'associer pour offrir une formation plus globale aux apprenti.e.s. Il arrive en effet régulièrement que certaines entreprises hésitent à former compte tenu de la spécificité de leur activité; elles ne pourraient enseigner tous les aspects d'un métier à leurs apprenti.e.s si l'on se réfère aux programmes de formation y relatifs. 
grille virilité/féminité. Ces normes de genre touchent tant l'apparence (il faut avoir «le physique de l'emploi») que les comportements et sont transmises durant la socialisation professionnelle (voir Chapitre 8). Ainsi, si au sortir du secondaire, certaines normes n'étaient pas encore intériorisées (Couppié et Epiphane, 2004), l'expérience en formation professionnelle y pourvoit, que ce soit par des encouragements, des brimades, des confrontations ou encore de la violence. La socialisation professionnelle n'est donc plus uniquement une habituation aux codes et normes d'une profession, d'une entreprise ou plus généralement du monde du travail, elle est aussi un apprentissage des normes de genre, qui prennent corps dans un système de rapports inégalitaires entre les sexes.

Dans les situations d'insertion atypique, ce processus peut encore être renforcé. En effet, l'apprenti.e en situation pionnière peine à s'identifier à un.e professionnel-le de référence et à travers elle ou lui, à une profession. Cette difficulté provient du fait que l'ensemble des codes (langage, actes symboliques) sont rattachés à des pratiques du groupe dominant, dont la personne pionnière se distingue en raison de son appartenance à un autre groupe de sexe. Difficile par exemple pour une jeune femme de se reconnaître dans le sobriquet " polymecs ", utilisé par les personnes qui travaillent en polymécanique pour se désigner entre elles; pourtant, cette désignation participe précisément de la construction d'une identité professionnelle valorisée (Castelli, Dransart et al., 2008). En outre, le rappel quasi permanent de son altérité ne permet pas à la personne en situation pionnière de se sentir membre à part entière d'un collectif de travail. Si dans le contexte helvétique il est particulièrement délicat pour les apprenti.e.s d'appartenir à un collectif ${ }^{22}$, elles et ils peuvent espérer à terme faire partie du collectif de travail constitué par leurs collègues. Or, les personnes pionnières dans leur formation, en particulier les filles affrontant les bastions masculins, n'y ont pas accès. Cette exclusion est d'autant plus dommageable que certains travaux ont souligné l'importance du collectif pour porter des revendications, faire entendre sa voix et faire reconnaître ses qualifications en déjouant les pièges des assignations de genre (D. Kergoat et al., 1992).

22 La nette prédominance de PME dans le tissu économique romand (et plus généralement dans les entreprises formatrices) fait que les apprenti.e.s sont fréquemment isolé.e.s (voir à ce propos Chapitres 6 et 7) et ne peuvent pas se constituer en collectifs d'apprenti.e.s, contrairement à ce qui est rendu possible par une insertion dans de grandes entreprises, disposant de centres de formation et donc d'un nombre important d'apprenti.e.s, comme l'a montré Prisca Kergoat pour la France (P. Kergoat, 2003). 
En situation pionnière, le rôle de la personne formatrice et des collègues est donc particulièrement important. L'intégration en situation atypique est réussie lorsque ces personnes de référence font une place à l'apprenti.e. Par contre, l'intégration pose problème, lorsque ces mêmes personnes favorisent la stigmatisation de la personne pionnière, en l'enfermant dans les frontières de sa catégorie de sexe. Fréquemment mises face à des représentations stéréotypées et dégradantes, les pionnières apprennent ce que signifie être une femme dans un univers professionnel masculin, où la résistance virile joue également sur la sexualité (Molinier, 2002b). L'intrusion de la sexualité au travail puise fréquemment dans les registres de la pornographie, où les femmes sont mises à disposition, instrumentalisées, dominées. Les jeunes femmes qui y sont soumises apprennent qu'elles ne sont pas considérées comme des professionnelles par les personnes de référence, elles l'enregistrent et doivent faire avec. Ainsi, c'est tout particulièrement dans l'interaction avec la personne formatrice, avec les collègues qu'elles prennent conscience de payer le prix fort de la transgression (Couppié et Epiphane, 2007; Marry, 2007).

\subsubsection{L'entrée en apprentissage ou la découverte de logiques de ségrégation et de discrimination}

L'entrée en formation professionnelle implique un changement majeur en matière d'environnement. Sil'école secondaire demeure, malgrél'organisation en filières, un espace où règne une certaine mixité, le travail fait entrer les jeunes femmes et les jeunes hommes dans des univers fortement ségrégués. Malgré les discriminations en vigueur dans l'enseignement (Baudelot et Establet, 1992c; Chaponnière et Chaponnière, 2006; Duru-Bellat, 1990 ; Mosconi, 1994; Zaidman, 1996), le secondaire se caractérise par son caractère mixte, y compris si la mixité formelle l'emporte sur la mixité réelle (Baudelot et Establet, 1992b). Cependant, l'entrée en formation professionnelle est marquée par un clivage strict: "séparés dès l'entrée, filles et garçons sont scolarisés à part dans des sections étanches, au point qu'on est en droit d'utiliser à leur propos l'expression 〈d'apartheid sexuel〉 employée par d'autres pour caractériser les conditions de travail faites aux hommes et aux femmes dans certaines entreprises» (Baudelot et Establet, 1992b : 159). En dépit des évolutions et du fait que depuis les années soixante, un certain sex-ratio est atteint (G. Moreau, 2000), la mixité des sections n'est pas garantie. En apprentissage dual, comme au lycée professionnel, les frontières entre métiers de filles et métiers de garçons demeurent hermétiquement et étonnamment stables: «l'apprentissage demeure un monde cloisonné 
ou filles et garçons se côtoient sans se rencontrer» (G. Moreau, 2000: 73 ; à ce propos, voir également OFS, 2013).

On retrouve en formation professionnelle la ségrégation horizontale du marché du travail, et «les clivages observés correspondent à la bonne vieille division entre les ‘qualités naturelles ‘ traditionnellement attribuées aux uns et aux autres : aux garçons, les professions productives de l'industrie et du bâtiment; aux filles, les soins du corps, de la couture, du ménage et du secrétariat» (Baudelot et Establet, 1992b : 160-162). Pour ces sociologues, une forte ségrégation intervient également dans les professions mixtes, à l'exception des métiers de bureau. Sexe, métier et genre se confondent ainsi inexorablement: «Une séparation matérielle aussi tranchée ne va pas, on s'en doute, sans engendrer chez les uns et les autres des comportements très particuliers à l'égard des différences de sexe. L'apprentissage d'un métier et l'appartenance à un sexe sont ici si étroitement et si «naturellement ` associés, qu'ils finissent par se confondre et ne plus faire qu'un; l'unité sexe-métier constitue alors pour les élèves un seul et unique principe d'affirmation de leur identité. D'où la tendance à multiplier et à accentuer, pour s'affirmer auprès des autres et de soi-même, les signes extérieurs et les attributs les plus visibles de son appartenance de sexe» (Baudelot et Establet, 1992b: 164).

Cette mise en contexte de la formation professionnelle en Suisse a permis d'esquisser quelques-uns des principaux enjeux qui la traversent. Dans une perspective de genre, le détour historique a permis de rappeler les résistances autour de la professionnalisation du travail féminin. Relire la naissance de la formation professionnelle à l'aune de ce débat est un moyen de mieux comprendre pourquoi les femmes en ont initialement été écartées. Ensuite, l'analyse de l'interdépendance entre le système de formation professionnelle, notamment dans sa forme duale, et le marché du travail, a permis de comprendre comment certaines inégalités, telles que la concentration ou la ségrégation, se maintiennent toujours sur le marché du travail (OFS, 2008b) et se répercutent sur le marché des places d'apprentissage. Ainsi, il ressort que les jeunes femmes subissent davantage les effets du décalage entre offre et demande de places d'apprentissage, et cela malgré un meilleur capital scolaire. Elles sont sur-sélectionnées pendant que leurs homologues masculins profitent d'une forme de sous-sélection, liée au plus grand nombre de professions dans lesquelles ils se répartissent. 
Les questions de transition relues dans une perspective de genre mettent également d'autres éléments en évidence: si le système dual permet une bonne transition entre école et monde du travail, ce n'est pas le cas pour les jeunes femmes, qui se retrouvent plus fréquemment sans solutions au sortir du secondaire. L'appartenance de sexe fait partie des facteurs sociodémographiques qui modèlent la transition, à l'instar de ce que d'autres travaux ont souligné. A partir d'analyses longitudinales, l'étude Transition école-emploi (TREE) a notamment identifié comme facteurs fragilisant les jeunes: le sexe (féminin), la filière suivie au secondaire I (filière à faible niveau d'exigences), le milieu social d'origine (familles défavorisées du point de vue socioéconomique), le statut migratoire (primo arrivant.e.s) (Meyer, 2004). Ces facteurs se retrouvent chez les personnes ayant un parcours délicat de même que chez celles qui optent pour des solutions transitoires $\left(10^{\mathrm{e}}\right.$ année, semestre de motivation - SEMO, etc.). Les filles sont par exemple plus nombreuses dans les structures de transition et y restent plus longtemps que les garçons, puisqu'on les retrouve non seulement dans les $10^{\circledR}$ années, mais aussi dans les $11^{\mathrm{e}}$ années (Amos, 2007; Meyer, 2004). Dès lors que le système de formation professionnelle dual est étroitement lié au marché du travail, et que ce marché s'organise notamment autour de la division des sexes, de leur hiérarchie et de la discrimination des femmes, les filles se retrouvent plus fragiles lors de l'entrée sur le marché des places d'apprentissage. Le sexe est donc bien un marqueur des conditions et de la qualité de la transition entre école et travail.

Enfin, la formation professionnelle s'avère constituer un lieu de reproduction et de production d'inégalités (Kergoat et Capdevielle-Mougnibas, 2015), et en particulier des normes de genre, sont acquises durant la socialisation professionnelle, notamment par la relation avec la personne formatrice et les collègues. Ainsi, la formation professionnelle permet d'apprendre un métier, mais également enseigne ce qu'est la masculinité et la féminité. Plus largement, la formation duale est un espace de production d'inégalités 


\section{Chapitre 2 \\ Le genre: angle mort de la littérature sur les arrêts en formation professionnelle}

Les arrêts prématurés en formation professionnelle renvoient intuitivement aux travaux sur le décrochage scolaire. Pourtant, la spécificité de la formation professionnelle suisse, en particulier sous sa forme duale, rend toute comparaison difficile ${ }^{23}$. En effet elle est structurellement différente d'une formation purement scolaire et renvoie à ce que Moreau (2000) appelle l'ambiguité fondatrice de l'apprentissage, soit le fait que l'apprenti.e doit composer avec deux mondes bien distincts: l'école et le travail. Ainsi, l'arrêt en formation professionnelle est caractérisé par une double rupture de contrat: celle du contrat scolaire et celle d'un contrat de travail. ${ }^{24}$

Si les travaux sur les résiliations de contrats d'apprentissage sont peu nombreux, les études sur le décrochage scolaire sont abondantes et s'appuient sur des approches diverses (économique, sociologique, psychologique ou pédagogique). Outre les facteurs liés à l'expérience scolaire de l'élève (faibles habiletés scolaires, retard ou échecs scolaires, mais aussi vécu négatif de l'école, indiscipline ou absentéisme), et qui se retrouvent peu ou prou dans les éléments participant d'un arrêt prématuré en formation professionnelle, les chercheur.e.s expliquent également le décrochage soit par des facteurs sociaux (familles, milieu social), soit par les particularités du système scolaire (taille de l'école,

23 L'enseignement post-obligatoire en Suisse (aussi appelé Secondaire II) offre trois possibilités de formation ou d'étude: la filière professionnelle, la filière de culture générale et la filière gymnasiale. La filière professionnelle, orientée vers la pratique, se déroule soit en entreprise (avec une partie en école professionnelle), soit en école de métiers (c'est-à-dire en école à plein temps). La formation peut s'échelonner sur 2 à 4 ans. Pour des informations plus détaillées sur l'enseignement post-obligatoire, en particulier sur le système de formation professionnelle Suisse, se reporter aux informations du SEFRI (2015).

24 Les offices de la formation professionnelle permettent toutefois à l'apprenti.e de résilier son contrat de travail tout en poursuivant sa formation en école professionnelle, ce qui lui laisse du temps (en général trois mois) pour chercher une nouvelle place de formation dans le même métier mais dans une autre entreprise. 
type de programmes, etc.) (Quiroga, Janosz et Marcotte, 2006). Pour sa part, Rosenthal (1998) regroupe les facteurs corrélés au décrochage en 12 catégories, dont un faible statut socioéconomique, l'appartenance à une minorité ethnique, le sexe, la qualité des relations familiales, l'absence de soutien social, l'absence d'engagement envers la formation et des facteurs liés à la "personnalité» (par exemple une faible estime de soi et une confiance en soi médiocre $)^{25}$. Relevons que ces facteurs sont très similaires à ceux qui interviennent dans les arrêts prématurés en formation professionnelle, comme nous le verrons ci-après.

Une grande hétérogénéité caractérise tant les processus amenant au décrochage scolaire, que les facteurs l'expliquant, si bien que certain.e.s auteur.e.s ont opté pour une approche par typologies. La même approche a été retenue par des chercheur.e.s s'intéressant à la formation professionnelle soit en dressant une typologie des jeunes ayant arrêtéleur formation et se retrouvant sans qualifications (Eckmann-Saillant et al., 1994), soit en proposant une typologie des modèles d'intégration dans le marché du travail de jeunes ayant arrêté leur formation professionnelle (Rastoldo et al., 2009).

Enfin, le décrochage scolaire a fait l'objet de quelques analyses en termes de genre. Elles soulignent la plus grande fragilité des garçons face à des filles qui font globalement état d'une meilleure réussite scolaire (Bouchard et St-Amant, 1996; Collectif, 2003; Marry, 2004a; Quiroga et al., 2006). L'étude des arrêts prématurés en formation professionnelle ne semble pas faire l'objet de telles analyses.

25 Il s'agit de préciser ici que si l'impact de facteurs tels que l'estime de soi et la confiance en soi sur le décrochage (scolaire ou en formation professionnelle) est indéniable, ils ne sont pas considérés dans la présente recherche comme des traits de personnalité. En effet, l'institution scolaire et les rapports sociaux, notamment de sexe, participent à produire tant l'estime de soi que la confiance en soi. Ainsi, après une socialisation secondaire où les filles ont appris quelle position elles occupaient dans la hiérarchie entre les sexes, après avoir expérimenté durant des années le caractère secondaire de leurs propos, de leurs interventions en classe (curriculum caché), elles quittent la scolarité obligatoire avec de bons résultats, mais une faible estime d'elles-mêmes et une confiance en soi médiocre. Il s'agit donc bien là d'une production sociale et non de traits individuels. D'autres catégories de personnes sont soumises à ces processus, par exemple les personnes migrantes. 


\subsection{La littérature sur les arrêts prématurés en formation professionnelle duale}

La formation professionnelle duale se distingue des autres filières de formation. Elle articule deux dimensions - la formation et la production - dans deux espaces distincts - l'école et l'entreprise. Il est indispensable de garder à l'esprit ces caractéristiques, car ces deux espaces n'interviennent pas au même titre dans l'explication de l'arrêt. Ainsi, ce dernier est-il souvent lié aux difficultés que les jeunes rencontrent sur leur lieu de travail; parfois c'est l'alternance entre la formation en école et celle en entreprise qui pose problème. Les décrocheur.e.s semblent être plus critiques vis-à-vis des formatrices et formateurs en entreprise qu'envers les enseignant.e.s des écoles professionnelles et jugent globalement plus positivement l'école que l'entreprise (Stalder et Schmid, 2006a). Le décrochage en formation professionnelle duale présente des configurations bien particulières - notamment l'articulation entre école et entreprise, ainsi qu'entre formation et production - qu'il s'agit d'investiguer de manière spécifique. Il ne peut pas se comprendre sans le cadre général dans lequel il s'inscrit: la transition entre l'école et le monde professionnel. L'arrêt est d'ailleurs envisagé ici comme signe de difficultés rencontrées lors de la transition (Masdonati, Lamamra et Jordan, 2010) et l'état de la littérature ne portera pas seulement sur des travaux questionnant les arrêts prématurés, mais également sur ceux abordant la question de la transition école - monde professionnel.

\subsubsection{Une recherche peu fournie, mais continue}

Les études sur l'arrêt prématuré de formation professionnelle en Suisse sont relativement rares. Les deux dernières vagues de recherches ont eu lieu au milieu des années 1990, puis à partir de 2005 (Ferron, Cordonnier, Schalbetter, Delbos-Piot et Michaud, 1997; Michaud, 2001; Neuenschwander et Stalder, 1998; Schmid et Stalder, 2007; Stalder et Schmid, 2006a). Dans les années 1990, l'accent a notamment été mis sur les effets d'un arrêt prématuré sur la santé des jeunes (Ferron et al., 1997), car l'objectif était de faire de la prévention, en particulier au niveau des dépendances, et de proposer des mesures d'accompagnement (COREL, 1999).

Les travaux des années 2000, privilégient une approche quantitative. Ils visent à mieux cerner l'ampleur du phénomène, à comprendreles raisons invoquées et à dresser une perspective plus globale, en croisant les points de vue des jeunes et des entreprises formatrices (Schmid et 
Stalder, 2007; Stalder et Schmid, 2006a). Cette dernière perspective se retrouve également dans les travaux qui proposent d'insérer le phénomène dans le cadre des transitions école-travail (Meyer, 2005; Rastoldo et al., 2009; Rastoldo et al., 2007). Plus récemment, la recherche du SRED ${ }^{26}$ (Rastoldo et al., 2009) s'est focalisée sur le parcours post-rupture. On retrouve ce questionnement chez Schmid (2010), qui s'intéresse à l'arrêt de formation comme «événement critique " (kritisches Lebensereignis) ${ }^{27}$. Plus rares sont les travaux qui ont opté pour une approche qualitative (Lamamra et Masdonati, 2009), qui permet de rendre compte de l'expérience vécue par les jeunes lors de leurs premiers pas en formation professionnelle et d'explorer ce qui se déroule au cours du processus conduisant à un arrêt prématuré.

Globalement, il ressort des enquêtes que le taux d'abandon varie entre 10 et $40 \%$ suivant la région considérée, et que la plupart des ruptures de contrat ont lieu durant la première année de formation. Par ailleurs, l'étude longitudinale Transition de l'école à l'emploi - TREE (Bertschy, Böni et Meyer, 2007; Meyer, 2005) montre que, six ans après la fin de l'école obligatoire, environ $10 \%$ des jeunes ont quitté le système de formation post-obligatoire sans avoir obtenu de diplôme. D'après les auteur.e.s, ce chiffre va vraisemblablement augmenter lors des prochaines années (Bertschy et al., 2007). En Suisse romande, le taux de rupture de contrat de formation professionnelle varie entre $11.7 \%$ pour le canton de Neuchâtel et 29.8\% pour Genève. En 2006, dans le canton de Vaud, on comptait $13.4 \%$ de résiliations de contrats de formation professionnelle (Stalder et Schmid, 2006b). Cependant, ces chiffres doivent être considérés avec prudence, car les méthodologies d'enquêtes varient d'un canton à l'autre, ce qui occasionne des différences importantes (voir supra) (Schmid, 2010). Plus que les taux, il faut souligner la relative stabilité du phénomène depuis une quinzaine d’années (Schmid, 2010).

\subsubsection{Les facteurs explicatifs}

L'importante variation des taux selon le secteur professionnel est un autre élément à considérer. Par exemple, dans le canton de Zurich, le taux de rupture de contrat dépasse $30 \%$ dans les métiers de la peinture en bâtiment, des soins corporels, de l'hôtellerie, de la restauration, ainsi

26 Il s'agit du Service de recherche en éducation du canton de Genève.

27 Evi Schmid développe cette notion à partir de la psychiatrie et de la psychologie clinique. Selon elle, il s'agit d'événements qui peuvent conduire à une réinterrogation du parcours de vie et permettre une réorientation (Schmid, 2010: 21-24). 
que dans les métiers artistiques (graphisme, décoration d'intérieur) (Neuenschwander et Stalder, 1998). D'autres facteurs influent sur le phénomène. Ainsi dans le canton de Berne (Stalder et Schmid, 2006a), le risque de rupture de contrat de formation professionnelle est plus élevé pour des jeunes issu.e.s d'une structure de transition, pour celles et ceux qui ont déjà interrompu une première formation du secondaire II, ainsi que pour les jeunes qui ont dû se résoudre à un métier ne correspondant pas à leur choix initial. Dans le canton de Genève, l'impossibilité de se former dans le métier souhaité et le fait d'être passé par une structure de transition avant l'apprentissage sont deux des plus importants prédicteurs de l'arrêt de formation professionnelle (Kaiser, Davaud, Evrard et Rastoldo, 2007). Ces constatations sont partiellement confirmées par les derniers chiffres de l'étude TREE: la probabilité d'être parmi les jeunes quittant prématurément le secondaire II est significativement plus élevée pour celles et ceux qui ont un bas statut socioéconomique, un faible niveau de compétences PISA en lecture ou ont eu recours à une structure de transition après l'école obligatoire. Cette probabilité est également plus élevée pour les personnes d'origine étrangère ou celles issu.e.s d'une filière du secondaire I à faibles exigences (Bertschy et al., 2007; Dubs, 2006). Cette dernière étude révèle également que le taux d'arrêt de formation professionnelle est plus élevé en Suisse romande. Les divers éléments qui influent sur les arrêts prématurés seront présentés en détail dans la troisième partie de ce chapitre. Un portrait sociographique des personnes qui interrompent leur formation professionnelle sera alors proposés à partir des éléments suivants: le secteur professionnel, le parcours antérieur (y compris le recours à une structure de transition), l'orientation par défaut (et le contexte plus général du marché des places d'apprentissage), le capital scolaire, le milieu social d'origine, la nationalité, et bien sûr le sexe et le type d'insertion dans l'emploi (traditionnelle ou atypique).

Sous l'angle du genre, il s'agit d'ailleurs de relever un paradoxe. Les travaux sur la transition entre l'école et le monde professionnel (Amos, 2007; Meyer, 2004) laissent apparaître le genre comme un facteur influençant ce passage. Or, s'il ressort que les femmes sont plus vulnérables lors de l'entrée sur le marché des places d'apprentissage (transition plus longue, plus chaotique, recours plus fréquent à des structures de transition et maintien plus long dans lesdites structures), ce phénomène n'apparaît pas dans les travaux sur les arrêts prématurés. Schmid (2010) affirme même qu'il n'y a pas ou quasi pas de différences entre femmes et hommes quant aux ruptures d'apprentissage. Cette 
conclusion est surprenante, puisque le passage par une structure de transition augmente le risque d'arrêt prématuré (Meyer, 2005) et que les filles sont plus nombreuses à passer par de telles structures. De plus, l'arrêt prématuré en formation professionnelle peut être compris comme le signe patent d'une transition qui aurait échoué (Masdonati et al., 2010; Lamamra et Masdonati, 2011a). Notons ici, qu'il y a très peu de matériel pour fonder une telle réflexion. En effet, les différences entre femmes et hommes apparaissent comme nulles ou très faibles dans la plupart des travaux sur les ruptures d'apprentissage (Neuenschwander, 1999; Schmid, 2010; Stalder et Schmid, 2006a) ${ }^{28}$.

Deux aspects semblent cependant se dégager de façon différente selon le sexe. En premier lieu, l'étude bernoise laisse entrevoir que les hommes sont plus touchés par un arrêt prématuré que les femmes. La différence est néanmoins minime: sur une moyenne d'environ 1500 résiliations annuelles, l'écart entre sexes est d'environ $1 \%$ par année d'entrée en formation ${ }^{29}$ (Stalder et Schmid, 2006). Dans l'étude genevoise, un constat analogue est posé: "Il s'agit plus fréquemment de garçons, $14 \%$ de l'ensemble des apprentis contre 11\% des apprenties» (Rastoldo et al., 2009: 13).

Le second élément où le sexe semble être une variable significative est la reprise après un arrêt prématuré (Rastoldo et al., 2009; Schmid et Stalder, 2007). Les filles se tournent plus fréquemment vers une formation supérieure à celle qu'elles ont quittée, s'inscrivant ainsi dans un parcours ascendant, tandis que les garçons optent pour des formations moins qualifiantes, se retrouvant alors dans un parcours de déclassement (Schmid et Stalder, 2007).

Les femmes et les hommes semblent donc être différemment touchés par la question de la transition d'une part (les femmes vivent un passage école - marché du travail plus délicat), par le décrochage d'autre part (les hommes semblent davantage touchés par les résiliations de contrats d'apprentissage) et enfin par les parcours post-arrêt (les femmes effectuant une mobilité ascendante et les hommes un déclassement).

28 Stalder et Schmid (2006a) font état de différences statistiques non-significatives, et Schmid précise que les différences sont nulles ou très faibles «Zwischen Frauen und Männern zeigen sich hingegen keine oder nur sehr geringe Unterschiede» (Schmid, 2010: 72).

29 «Männer sind etwas häufiger von einer Vertragsauflösung betroffen als Frauen. Die Geschlechtsunterschiede sind jedoch gering (Unterschied rund $1 \%$ pro Eintrittsjahrgang)» (Stalder et Schmid, 2006a). 


\subsubsection{Les principales raisons recensées}

Dans les travaux sur les arrêts en formation professionnelle, les raisons évoquées sont multiples (Stalder et Schmid, 2006a) et peuvent se regrouper en deux catégories. La première catégorie comprend des éléments tels que l'orientation professionnelle au sens large. En effet, la question du choix d'une profession peut être liée à la maturité vocationnelle des individus (instabilité des intérêts, méconnaissance de ses aptitudes, fausses représentations du métier) ou aux places disponibles correspondant à leurs intérêts. La question du choix du métier ou de l'entreprise ne dépend pas uniquement de facteurs individuels, mais doit également être rapportée à l'accès aux places dans un marché des places d'apprentissage difficile (Herzog, Neuenschwander et Wannack, 2004; Rastoldo, 2006). Celui-ci est tributaire des procédures utilisées par les entreprises dans la sélection des futur.e.s apprenti.e.s et fréquemment fondées sur des critères de sélection peu prévisibles et hétérogènes (Imdorf, 2007b). La seconde catégorie regroupe des raisons liées aux conditions de formation et de travail rencontrées par les apprenti.e.s, notamment aux compétences pédagogiques des personnes formatrices, à un climat peu encourageant dans l'entreprise, à un manque de liens entre école professionnelle et formation en entreprise, à l'attribution de tâches monotones ou sans lien direct avec le métier, à une surcharge de travail à l'école ou en entreprise, ainsi qu'à des questions de salaire ou de temps de travail (Stalder et Schmid, 2006a, 2006b).

S'ajoutent enfin des raisons qui ne sont liées ni avec le choix d'entreprise ou de métier, ni avec les conditions rencontrées dans l'exercice de l'activité, ni à la formation professionnelle en tant que telle, et que l'on retrouve dans l'étude de Evi Schmid (2010) sous «raisons personnelles ». Ces motifs (relations familiales, dépendances, etc.) sont liés aux autres sphères de vie des adolescent.e.s et mettent en évidence l'étroite imbrication entre elles ${ }^{30}$.

L'une des enquêtes suisses les plus récente en matière d'arrêt de formation professionnelle est l'étude Lehrvertragsauflösungen (LEVA), menée dans le canton de Berne (Schmid et Stalder, 2007; Stalder et

30 Une remarque quant à l'utilisation de cette catégorie «personnelle » s'impose ici. En effet, elle est probablement issue des documents cantonaux à remplir lors d'une résiliation de contrat d'apprentissage. Il s'agit en effet de signaler les motifs de la rupture du contrat et tout ce qui n'a pas directement trait à la formation est ainsi classé sous «raisons personnelles». Selon les cantons, ces raisons ne sont pas davantage précisées et par la suite, les chercheur.e.s n'investiguent pas nécessairement plus loin. 
Schmid, 2006a). Cette étude relève notamment que patron-ne-s et ex-apprenti.e.s expliquent différemment la rupture du contrat de formation. Les patrons évoquent, plus souvent que les jeunes, un manque d'efforts et de mauvaises performances à l'école. Pour leur part, les ex-apprenti-e.s dénoncent davantage les mauvaises conditions de travail et de formation ainsi que l'existence de situations conflictuelles au sein de l'entreprise. Les auteures relèvent que les raisons évoquées pour expliquer l'arrêt de formation sont plus nombreuses chez les ex-apprenti.e.s que chez les patron-ne.s. Les résultats étant non-significatifs en ce qui concerne l'analyse de la variable sexe (Stalder et Schmid, 2006a), il est impossible de savoir si certaines raisons sont plus fréquemment invoquées par les hommes ou les femmes. Le dernier rapport de la recherche bernoise (C. Moser, Stalder et Schmid, 2008) aborde d'autres types de raisons, telles que la discrimination institutionnelle ${ }^{31}$, la question du réseau social ou encore les effets des préjugés et des stéréotypes. Les chercheur.e-s le font en ce qui concerne les migrant.e.s, mais ce type de réflexion pourrait être élargi àl'analyse des groupes de sexe. Des éléments similaires apparaissent lorsqu'une perspective de genre est adoptée et l'on voit apparaître d'autres formes de discriminations institutionnelles : ségrégation horizontale et/ ou verticale, concentration, division sexuelle du travail (voir Chapitre $1^{\text {er }}$, ainsi que Chapitre 3 et Chapitre 8) ou encore procédures de sélection des apprenti.e.s par les entreprises (Imdorf, 2004). Une autre discrimination, que l'on pourrait qualifier de discrimination indirecte, mais pas traitée sous cet angle dans la littérature, serait l'impact de la filière suivie au secondaire I sur la sélection. Filles et garçons ne fréquentent pas les mêmes filières, les garçons étant tendanciellement davantage insérés dans des filières du secondaire moins exigeantes ${ }^{32}$.

31 Par discrimination institutionnelle les deux autrices se réfèrent à l'impossibilité d'accès à un éventail de professions, nécessitant une formation secondaire de bon niveau, ce que ces jeunes issu.e.s de la migration ont moins fréquemment que les Suisses.ses (C. Moser et al., 2008).

32 Les statistiques vaudoises ne permettent pas d'affirmer cette assertion. Il est en effet impossible de connaître la répartition par sexe dans les différentes filières du secondaire I. Cependant, deux indices permettent d'aller dans ce sens : la meilleure réussite scolaire des filles (Couppié et Epiphane, 2004; Mosconi, 1994) et les filières privilégiées pour le secondaire II : la formation professionnelle pour les garçons (nécessitant un capital scolaire moyen), les écoles de formation générale pour les filles (nécessitant un capital scolaire relativement élevé) (Meyer, 2004). 


\subsubsection{Des répercussions variées}

Plusieurs enquêtes datant des années 90 (Ferron etal., 1997; Neuenschwander, 1999; Neuenschwander, Stalder et Süss, 1996) révèlent que l'arrêt de formation professionnelle se corrèle avec des problèmes de santé physique ou psychique des apprenti.e.s. Comparé.e.s à leurs collègues en formation, les jeunes qui interrompent leur apprentissage sont davantage exposé.e.s à des comportements à risque, comme la consommation de drogues, d'alcool et de tabac. Elles et ils manifestent aussi davantage de symptômes dépressifs, d'anxiété ou des idées voire des conduites suicidaires (pour plus de détails, voir Chapitre 6).

L'étude LEVA (Stalder et Schmid, 2006a) montre que le fait d'arrêter prématurément sa formation provoque de la tristesse, un sentiment d'échec, de la colère et une mauvaise conscience chez les ex-apprenti.e.s, et, d'autre part, que l'intensité de ces sentiments change suivant la raison de l'arrêt. Des interruptions motivées par de faibles performances ou des problèmes de santé provoquent des sentiments fortement négatifs, alors que ceux imputés aux mauvaises conditions de travail ou de formation ainsi qu'à un choix inapproprié de métier ou d'entreprise peuvent au contraire provoquer des sentiments positifs, tels que la joie, le soulagement. Le vécu dépend également de la situation au moment de l'arrêt. Les jeunes qui ont retrouvé une place (en ayant ou non changé de métier) sont soulagé.e.s d'avoir arrêté leur formation et se sentent plus satisfait.e.s de leur vie, alors que les personnes qui n'avaient pas de solution de rechange lors de la rupture du contrat ou qui ont dû revoir à la baisse leur niveau de formation (déclassement) tout en restant dans le même secteur professionnel sont davantage déçues.

Les personnes sans solution au moment de l'arrêt sont dans une situation délicate du point de vue psychologique, comme on l'a vu ci-dessus (difficulté à surmonter la crise provoquée par l'arrêt de formation, sentiment de blocage), mais aussi du point de vue social (risque de chômage, de chômage de longue durée, situation de travail précaire, absence de perspectives d'avenir, problèmes de santé, manque de ressources sociales). Larrêt de formation compromet donc parfois l'insertion socioprofessionnelle des jeunes. Si quatre jeunes sur cinq arrivent à retrouver une place de formation, cette probabilité est moins bonne pour les jeunes à faible statut socioéconomique ou d'origine étrangère. S'ajoute à cela le facteur temps. En effet, plus le temps passe après un arrêt, plus les personnes se retrouvent en situation de recherche d'emploi ou d'emploi non qualifié (Rastoldo et al., 2009). Les emplois obtenus sont souvent précaires et regroupés dans un éventail assez limité: «A l'absence de 
qualification scolaire correspond donc chez ces jeunes travailleurs des accès à l'emploi dans des domaines restreints, parfois flous, relevant le plus souvent de tâches d'exécution, probablement en nombre diminuant (en comparaison avec les secteurs de croissance d'emploi) et dont les perspectives ne semblent pas orientées vers une requalification (sauf peut-être dans la vente)»(Rastoldo et al., 2009: 34).

\subsubsection{Des parcours diversifiés}

L'étude LEVA montre que les jeunes ayant interrompu leur formation professionnelle prennent des chemins divers (Schmid et Stalder, 2007). Presque la moitié des personnes interrogées avaient trouvé une solution avant de rompre leur contrat. La majorité de celles-ci poursuivent une formation dans le même métier, en changeant d'entreprise, ou se réorientent vers une formation dans le même secteur professionnel mais qui demande un autre niveau d'exigence. L'autre moitié des jeunes n'avait pas trouvé de solution lors de la rupture du contrat. La plupart avait déjà interrompu une formation auparavant et avait dû faire des compromis quant à la profession choisie. Plusieurs indicateurs montrent que ces personnes diffèrent de celles ayant retrouvé une place avant l'arrêt: elles sont davantage mécontentes du travail à effectuer et de la formation reçue, présentent un cumul de raisons expliquant l'arrêt, manifestent davantage de souffrance physique, ont une plus grande consommation de tabac ou de médicaments et sont globalement moins satisfaites de leur vie. De plus, le taux de jeunes réintégrant une formation suite à une interruption prématurée n'augmente que très lentement avec le temps, ce qui confirme l'importance du facteur temps mise en évidence par les études des années 90 . Ainsi, 18 mois après l'arrêt, un·e jeune sur trois n'a toujours pas retrouvé de place de formation qualifiante. Ce tiers, réunit particulièrement des jeunes d'origine étrangère et qui ont déjà interrompu une formation, ainsi que des jeunes issu.e.s de la formation élémentaire. Ce qui semble déterminant sur ce point, c'est la capacité et la possibilité de solliciter de l'aide durant et après l'arrêt.

La diversité des parcours apparait également dans l'étude menée par le SRED (Rastoldo et al., 2009). Dans leur rapport, les auteurs soulignent les trois types de parcours suivant un abandon d'apprentissage : le retour en formation, l'accès à l'emploi et enfin d'«autres situations », qui intègrent notamment congé sabbatique, service militaire et chômage ${ }^{33}$.

33 Rastoldo et al. (2009) ont regroupé dans cette catégorie «autres situations» des situations hétérogènes. On y trouve des jeunes qui ne sont ni en emploi, 
Si dans un premier temps, le retour en formation regroupe une majorité de jeunes, cette prédominance s'érode au fur et à mesure que la période s'allonge $^{34}$. Sur l'ensemble de la période étudiée, on assiste également à une augmentation, puis à une stabilisation des «autres situations». Les auteurs relèvent que si l'emploi progresse, il est fréquemment précaire, peu qualifié. En outre, du point de vue du genre, si Schmid (2010) affirme que les chances de reprise de formation ("Wiedereinstieg») sont similaires pour les filles et les garçons, tant l'étude LEVA (Schmid et Stalder, 2007) que celle du SRED (Rastoldo et al., 2009) montrent que la situation diffère selon le sexe: les filles s'en sortent plus favorablement que les garçons. Elles reprennent en effet plus fréquemment une formation supérieure à celle qu'elles ont quittée et s'inscrivent ainsi dans un mouvement ascendant, alors que les garçons sont surreprésentés dans les parcours de déclassement (Schmid et Stalder, 2007). Par ailleurs, les filles insérées dans des formations à haut niveau d'exigences ont plus souvent tendance à reprendre une formation en école à plein temps, alors que les garçons insérés dans les filières à faible niveau d'exigence reprennent surtout un apprentissage dans le système dual (Rastoldo et al., 2009). Il est possible que le choix des filles, l'école à plein temps, soit consécutif aux difficultés particulières qu'elles ont rencontrées dans leur premier contact avec le marché du travail (ségrégation, discriminations, division sexuelle du travail; voir Chapitre $1^{\text {er }}$. Reprendre une formation dans un système à plein temps leur permet peut-être d'échapper provisoirement à ces éléments. Parallèlement, le maintien des garçons dans le système dual, en particulier ceux inscrits dans des apprentissages à faible niveau d'exigences (Rastoldo et al., 2009), met en évidence certains enjeux du choix de la formation professionnelle, soit la revalorisation via l'activité professionnelle de personnes ayant un lourd passé scolaire (voir Chapitre 3). Le choix de filières différenciés renvoie à la manière dont filles et garçons abordent l'entrée dans le monde du travail, puis dont elles et ils gèrent, une fois en formation, la tension entre apprendre et produire mise en évidence par Moreau (2000).

ni en formation et qui ne recherchent ni l'un ni l'autre. En plus des exemples cités, il peut s'agir de personnes en voyage, en séjour linguistique ou encore momentanément privées de liberté.

34 Plus le temps passe, moins on trouve de jeunes en situation de «formation». D'une part, parce que les formations entreprises juste après l'arrêt arrivent à leur terme et d'autre part, parce qu'à terme, les personnes privilégient une insertion dans l'emploi, qu'il soit qualifié ou non. 


\subsection{Portrait sociographique des jeunes qui arrêtent leur formation professionnelle}

Les diverses études sur les arrêts d'apprentissage mentionnent un certain nombre de facteurs sociobiographiques qui influencent le processus (C. Moser et al., 2008; Rastoldo et al., 2009; Schmid, 2010 ; Schmid et Stalder, 2007; Stalder et Schmid, 2006a). Ces facteurs vont être repris en détail ici et complétés, lorsque cela est possible, par certaines hypothèses issues de l'analyse qualitative (Lamamra et Masdonati, 2009).

Pour autant, le projet n'est pas d'établir un profil des «jeunes à risque», comme le proposait l'étude de Eckmann-Saillant et al. (1994) pour les jeunes sans qualification du canton de Genève. Une partie de ces jeunes avaient préalablement interrompu une formation professionnelle, ce qui permet de faire la comparaison avec la population de la présente étude. Un élément mis en évidence par cette recherche sera cependant souligné : à savoir l'hétérogénéité du groupe des jeunes sans qualification $^{35}$, aussi bien en termes de position sociale que d'attitudes et de stratégies déployées face à l'absence de formation. Si pour certains groupes de personnes (jeunes issu·e.s de familles précaires, immigrée.es récent.e.s, conformistes passifs et passives), l'absence de qualifications est un facteur de vulnérabilité (Castel, 1995), voire d'exclusion socioprofessionnelle durable, ce n'est pas le cas pour toutes et tous.

\subsubsection{Les secteurs professionnels}

Le secteur d'activité semble jouer un rôle déterminant sur les risques de résiliation. En effet, certaines professions connaissent des taux de rupture élevés. Il serait intéressant d'avoir des données qualitatives qui permettraient d'affiner les connaissances des problématiques spécifiques à chaque secteur, en termes de conditions de formation, de contraintes, de pénibilité, d'environnement de travail, etc. Toutefois, commela recherche a fait le choix méthodologique d'une approche intersectorielle, le nombre de cas est trop faible pour proposer des interprétations pertinentes.

En outre, il faudrait conduire plusieurs analyses pour appréhender les professions qui connaissent des taux élevés de ruptures, tant les diffé-

35 Il est important d'insister sur cette hétérogénéité, car elle explique pourquoi aborder la question de l'arrêt prématuré en formation professionnelle en termes de « jeunes à risque » n'est pas pertinente. Et s'il semble indispensable d'étudier les facteurs ayant une influence sur le processus de décrochage, il est impératif que ceux-ci ne soient pas abordés comme des «facteurs prédictifs », sur le modèle d'enquêtes «épidémiologiques». 
rences entre elles sont importantes. Les importants taux de rupture sont liés dans certaines professions à la pénibilité (charges, horaires, travail en plein air) à l'instar des métiers de l'hôtellerie et du bâtiment. Dans d'autres professions - plus rarement associées à la pénibilité, comme les professions commerciales - des facteurs plus subjectifs interviennent. Les emplois de bureau étant considérés comme plus faciles (en termes de contraintes physiques), il semble que lorsque les conditions de travail y sont difficiles, la situation est moins bien tolérée: «D’une certaine façon, c'est un peu comme si les aspects pénibles étaient moins acceptables dans le commerce que dans la construction " (Rastoldo et al., 2008: 31).

Le devenir des jeunes après un arrêt diffère d'un secteur à l'autre. Dans le commerce par exemple, une grande partie des jeunes changent de niveau d'exigence suite à un arrêt d'apprentissage (promotion, déclassement), alors que dans la construction, un nombre important de jeunes se trouve sans solution de rechange (Schmid et Stalder, 2007). Les significations de ces arrêts ne sont donc pas du tout identiques (Cart et Toutin, 2015; Duc et Lamamra, 2014).

Conjointement à la question des secteurs professionnels, se pose la question du niveau de formation professionnelle. Le taux de résiliation est plus important dans les professions à faible niveau d'exigences ${ }^{36}$ que dans celles à niveau d'exigences moyen ou élevé, (Rastoldo et al., 2009; Stalder et Schmid, 2006a). Ce phénomène semble même s'être accru ces dernières années: des branches telles que la coiffure, la cuisine ou la maçonnerie connaissent une progression de leur taux de résiliation (Stalder et Schmid, 2006a). L'étude genevoise souligne également que les jeunes en formation élémentaire (aujourd'hui formation initiale en deux ans) interrompent davantage leur apprentissage. Ce type de formation professionnelle faisant suite à un passé scolaire souvent difficile, l'arrêt s'inscrit dans le prolongement d'un parcours déjà chaotique.

\subsubsection{La nationalité}

La nationalité joue un rôle déterminant dans la résiliation de contrats d'apprentissage. Indépendamment du secteur professionnel, les jeunes d'origine étrangère dénoncent plus souvent leur contrat avant terme que

36 La classification des niveaux d'exigence en trois niveaux d'exigences, faible, moyen et élevé a été proposé par le Service d'orientation cantonal du canton de Berne et reprise par l'équipe du SRED dans leurs différents travaux sur la formation professionnelle (Rastoldo et al., 2009; Rastoldo et al., 2007). 
les jeunes suisses (Stalder et Schmid, 2006a; Rastoldo et al., 2009) ${ }^{37}$. La tendance est plus marquée encore pour les allophones (Rastoldo et al., 2009) ${ }^{38}$. Il ressort par ailleurs de l'étude LEVA (C. Moser et al., 2008) que les motifs évoqués pour les jeunes d'origine étrangère sont centrés sur les résultats obtenus à l'école professionnelle ${ }^{39}$. Cela en particulier pour les jeunes originaires des Balkans, de Turquie et du Portugal (C. Moser et al., 2008). Enfin, divers travaux rendent compte des stéréotypes et des discriminations en vigueur sur le marché du travail et de la formation professionnelle (Eckmann-Saillant et al., 1994; Fibbi, Kaya et Piguet, 2003; Imdorf, 2007b; Meyer, 2004; Pfister Giauque et Flamigni, 2010).

La nationalité influe également sur le réseau mobilisable après un arrêt prématuré de formation, notamment lors de la recherche d'une nouvelle place d'apprentissage: les apprenti.e.s d'origine étrangère sont plus fréquemment livré.e-s à elles- et eux-mêmes que les jeunes suisses.ses. Elles et ils trouvent moins de soutien non seulement auprès de leurs parents, mais également de la commission de surveillance des apprentissages (C. Moser et al., 2008). Il s'avère donc qu'en plus d'un réseau personnel déficient (parents n'ayant pas fait leur formation en Suisse, ne connaissant pas nécessairement son fonctionnement, voire n'étant pas inséré·e.s professionnellement), ces jeunes sont pénalisé.e·s par le réseau institutionnel.

\subsubsection{Capital scolaire et parcours antérieur}

Le parcours antérieur (filière suivie au secondaire, réorientation, redoublement, passage par une structure de transition, déroulement de la transition) et le capital scolaire influencent le processus qui conduit à un arrêt prématuré. Les personnes avec les parcours les plus chaotiques, soit celles qui ont passé par une structure de transition (Rastoldo et al., 2009; Stalder et Schmid, 2006a), celles qui ont connu une précédente interruption de leur formation dans le secondaire II (Stalder et Schmid, 2006a) ou encore celles qui ont vécu une période sans formation (Schmid

37 L'étude bernoise observe un écart significatif: si du côté des jeunes d'origine étrangère, on connaît une résiliation pour trois contrats, ce taux diminue pour les jeunes d'origine suisse à un pour cinq. Dans l'étude genevoise, les chiffres sont similaires: $16 \%$ de taux de résiliation chez les jeunes d'origine étrangère pour $11 \%$ chez les jeunes d'origine suisse.

38 Dans l'étude genevoise, le taux de résiliation est de $15 \%$ pour les étrangères et étrangers allophones contre $12 \%$ pour personnes d'origine étrangère, mais francophones.

39 Ce motif de résiliation fait consensus et apparaît tant chez les jeunes que chez leurs employeur.e.s. 
et Stalder, 2007), ont davantage de risques d'interrompre leur formation professionnelle. L'étude genevoise signale également les personnes qui ont obtenu une attestation de formation élémentaire ${ }^{40}$ avant d'entamer un CFC, ce qui, pour les chercheur.e.s du SRED (Rastoldo et al., 2009) est un signe clair de difficultés scolaires antérieures. Le capital scolaire et le parcours antérieur jouent ainsi un rôle déterminant sur l'ensemble du processus.

Ces deux facteurs influencent en premier lieu l'accès à une place d'apprentissage, car les entreprises privilégient les jeunes issu.e.s des filières les plus exigeantes, lorsqu'elles ont le choix (Dubs, 2006; Imdorf, 2004 ; Meyer, 2004; U. Moser, 2004). En deuxième lieu, ils pèsent sur les modalités de l'arrêt: les personnes dotées du meilleur capital scolaire, comme celles ayant un parcours antérieur linéaire, sont plus fréquemment à l'initiative de la décision. Pour elles, l'interruption est choisie et de ce fait fréquemment bien vécue. Troisièmement, ces facteurs marquent tout le processus qui conduit à l'arrêt, les personnes ayant un parcours non-linéaire vivant plus difficilement ces nouvelles difficultés. Elles signalent ainsi plus fréquemment de la souffrance et vivent mal l'arrêt. Enfin, le sens qui est donné à cette expérience est également marqué par le parcours scolaire. La signification d'un arrêt pour une personne à faible capital scolaire est plus dramatique et s'apparente davantage à une rupture, compte tenu du fait que ses chances de retrouver une nouvelle place sont moins bonnes que pour les personnes dotées d'un capital scolaire élevé (Imdorf, 2007b; Meyer, 2003, 2004).

\subsubsection{Contexte du marché des places d'apprentissage et choix par défaut}

La manière dont la formation a été choisie a un impact sur la poursuite de celle-ci. Les personnes ayant déjà interrompu une formation ou vécu précédemment une phase sans formation sont aussi celles qui doivent se montrer les plus flexibles au moment du choix d'un apprentissage. Selon

40 Cette formation est destinée aux jeunes ayant des difficultés (scolaires ou autres). Elle leur permet de faire valoir des habiletés pratiques et d'exercer une activité professionnelle. Elle est sanctionnée par un Certificat de Formation Professionnelle-CFP. Elle est remplacée progressivement par la «formation initiale en deux ans", dont l'accès est légèrement plus exigeant et qui est sanctionnée par une attestation reconnue au niveau fédéral (l'Attestation Fédérale de Formation Professionnelle - AFP). La mise en œuvre de la nouvelle formation en deux ans n'ayant pas encore eu lieu dans l'ensemble des professions (l'élaboration des nouvelles ordonnances étant en cours), les deux formations coexistent. 
l'étude LEVA (Schmid et Stalder, 2007), près d'une personne sur trois dans cette situation a dû se rabattre sur un métier ou une entreprise qui ne correspondait pas à ses souhaits, ce que Rastoldo (2006) qualifie de choix non maîtrisé et qui a été qualifié ici de choix par défaut. En ce cas, les facteurs sociobiographiques se cumulent avec le contexte du marché des places d'apprentissage (Stalder et Schmid, 2006a). Or, ce type de choix influence négativement les chances de se maintenir en formation (Rastoldo et al., 2009). Lorsque le métier a été retenu par défaut, les apprenti.e.s semblent avoir plus de peine à supporter les difficultés et les contraintes de la profession. Dans la continuité de ce choix marqué par l'hésitation ou la résignation, «les sentiments de ne pouvoir agir sur sa destinée, d'être empêché par autrui de faire ce que l'on veut, ou encore que le moment du choix d'orientation est encore à venir sont des sentiments plus fréquemment ressentis par ceux qui interrompent leur formation avant terme» (Rastoldo et al., 2009).

\subsubsection{Milieu social à faible statut socio-économique}

Divers travaux évoquent l'impact du milieu social sur le déroulement de la transition. En ce qui concerne les arrêts prématurés en formation professionnelle, l'étude du SRED (Rastoldo et al., 2009) relève que les jeunes décrocheur.e.s appartiennent plus fréquemment à la catégorie sociale "autre et sans indication", ce qui peut signifier que ces personnes sont issues d'un milieu social particulièrement défavorisé ou que l'information sur l'origine sociale est inconnue parce qu'elles sont récemment arrivées dans le système de formation genevois, en particulier pour effectuer leur apprentissage (migration récente).

Le rôle du milieu social apparaît également dans la présente étude. Les jeunes de milieux ouvriers invoquent plus fréquemment des difficultés d'apprentissage du métier comme raison de l'arrêt, à savoir des problèmes de performances scolaires ou un cadre de formation inadéquat. En ce qui concerne le premier point, une hypothèse peut être que l'accès à des soutiens est plus difficile dans ce milieu (Bourdieu et Passeron, 1970; Duru-Bellat, 2002), tant sur le plan des appuis concrets (aide aux devoirs, financement de cours de rattrapage, etc.) que sur le plan des ressources symboliques (réseaux pour trouver des soutiens, notamment institutionnels). Quant au cadre formatif, on peut faire l'hypothèse suivante: le réseau familial étant moins étendu, il ne permet pas d'accéder aux entreprises les mieux dotées du point de vue de la formation et de l'encadrement. Concernant l'encadrement, il faut également signaler les difficultés rencontrées par certain·e.sjeunes issu.e.s 
de milieu populaire face à la hiérarchie professionnelle. En effet, dans certains cas, on peut véritablement parler d'une socialisation familiale aux rapports de classes (méfiance du milieu de travail, des rapports de hiérarchie, etc.) (P. Kergoat, 2006).

Face aux difficultés, les personnes issues de milieu ouvrier évoquent avant tout de la souffrance éthique, mais ne parlent pas de souffrance mentale. Cette constatation n'est guère surprenante pour un milieu qui valorise parfois des attitudes héroïques et des stratégies collectives visant à oublier la peur et la souffrance (Dejours, 2000b). Ainsi, seul l'aspect éthique peut être évoqué, car il fait écho à des valeurs professionnelles et à un savoir-faire ouvrier qui ne peut se réaliser (P. Kergoat, 2006). Ces jeunes se distinguent encore par leur vécu positif de l'arrêt de formation. Cette réaction semble étonnante de la part de jeunes pour qui la formation professionnelle est l'occasion d'une ascension sociale, leurs parents exerçant fréquemment une profession non-qualifiée. Si l'arrêt de formation met un terme à l'espoir de pouvoir échapper à la condition ouvrière (P. Kergoat, 2003), l'expérience professionnelle de la famille fonctionne parallèlement comme élément rassurant, puisqu'elle illustre la possibilité de "s'en sortir » sans formation. Ainsi, bien qu'ayant pleinement conscience de l'importance du CFC sur le marché du travail, les jeunes des milieux populaires valorisent aussi «la débrouillardise » (Eckmann-Saillant et al., 1994).

\subsubsection{Sexe}

Certains travaux soulignent que les garçons sont davantage concernés par les arrêts en formation professionnelle (Rastoldo et al., 2009), ce qui est peut-être la conséquence d'un parcours scolaire plus difficile. En effet, la socialisation masculine se construit notamment sur un refus de se soumettre aux normes del'institution scolaire. On peut fairel'hypothèse que cette forme d'insubordination se retrouve lors de l'expérience en formation professionnelle, se déplaçant du rejet de l'institution scolaire au refus de l'autorité patronale et de la hiérarchie de l'entreprise (P. Kergoat, 2006). Et sans doute est-elle plus marquée chez les jeunes hommes issus de milieux ouvriers, qui ont intériorisé une méfiance de classe à l'égard du monde du travail et des rapports de hiérarchie (P. Kergoat, 2006).

La perspective de genre étant au cœur de cette étude, cette dimension (et donc la variable sexe) sera amplement développée dans la suite. En effet, l'analyse s'intéressera aux différents endroits où se jouent les rapports sociaux de sexe durantl'expérience de formation professionnelle. 


\subsubsection{Insertion pionnière}

Les travaux récents sur les interruptions prématurées en formation professionnelle ne soulèvent pas la question des professions atypiques. Pourtant, le parcours des personnes en situation pionnière présentent certaines singularités. Ainsi, les filles ayant opté pour une profession masculine connaissent un taux plus élevé d'arrêts prématurés que les garçons dans les professions féminines (Rédaction, 2008) et que la population apprentie dans son ensemble. Dans la présente étude, les pionnières invoquent plus fréquemment que les autres des conditions de travail difficiles pour expliquer l'arrêt de leur formation. Elles parlent davantage de souffrance, y compris physique. Outre la pénibilité de certains métiers «masculins», ce phénomène met en évidence les situations de rudesse ou de mises à l'épreuve dont peuvent être victimes les pionnières (Legault, 2001). Lorsque les garçons quittent une formation atypique, ils le font pour d'autres raisons que la souffrance, notamment le manque de perspectives, les conditions de rémunération et la faible reconnaissance sociale de professions fortement féminisées (Croisier, 2002; Thiébaud, 2004).

Si les femmes en situation atypique interrompent plus fréquemment leur apprentissage, elles sont cependant moins souvent à l'origine de la résiliation. Elles subissent cette décision ou essaient de négocier avec leur employeur. Cette forte persévérance peut s'expliquer par les difficultés d'accès à une place d'apprentissage dans un métier atypique (Croisier, 2002; Imdorf, 2004), ainsi que par les objections del'entourage (Thiébaud, 2004; Vouillot, 2007). Y mettre un terme pourrait valider les résistances et confirmer les pronostics négatifs. La modalité de l'arrêt (arrêt subi) a un impact sur le vécu de celui-ci. Ainsi, les pionnières vivent plus fréquemment cet événement de manière négative, en comparaison avec les personnes en situation d'insertion traditionnelle. Arrêter une formation atypique signifie en effet invalider une orientation, qui a de fait constitué une véritable transgression (Vouillot, 2007). Pour certaines, ce moment signifie également devoir abandonner leur envie de mobilité de genre (Guichard-Claudic et al., 2008).

Il est probable ${ }^{41}$ que les garçons ne le vivent pas comme un événement négatif, dans la mesure où en quittant une formation dans un métier atypique, ils réintègrent leur position dans les rapports sociaux

41 Nos données sur les garçons en situation atypique sont trop faibles $(\mathrm{N}=2)$ pour pouvoir affirmer qu'ils auraient des modalités d'arrêt ou qu'ils vivent cet événement de manière spécifique, d'où l'usage du conditionnel. 
de sexe. Ils reviennent ainsi vers une orientation qui leur permet de tenir leur rôle de pourvoyeur principal («male breadwinner»). En outre, les garçons s'orientent fréquemment dans des professions atypiques par accident (Lemarchant, 2007), comme l'illustre les deux pionniers de cette étude, ce qui atténue l'enjeu de transgression évoqué ci-dessus pour les filles. Par contre, la question de la mobilité de genre se pose différemment, choisir une profession traditionnellement féminine pour un garçon, c'est faire un mouvement descendant, alors que le choix, par une femme, d'une profession majoritairement occupée par des hommes constitue un mouvement ascendant.

Cet état des lieux a mis en évidence un taux de résiliation de contrat relativement élevé (entre 10 et $40 \%$ ). Ce dernier varie selon les régions et les professions, mais reste stable depuis une quinzaine d'années. Les résiliations de contrat interviennent en majorité durant la première année de formation professionnelle et les raisons invoquées sont généralement multiples. Ainsi, une personne arrête pour un cumul de raisons et non pour un seul motif ${ }^{42}$.

Par ailleurs, l'arrêt de formation n'est pas un événement soudain, mais l'issue d'un processus (Schmid, 2010), qui peut commencer bien avant l'entrée en apprentissage. En effet, divers facteurs sociaux influencent les risques d'arrêter (le milieu social, le parcours scolaire, la nationalité, le sexe, etc.).

En outre, l'arrêt peut être vécu comme un événement positif ou comme une véritable rupture, notamment dans le processus plus général d'insertion. Le sens qu'il prend influencera le parcours ultérieur. Toutefois, si un arrêt peut être précarisant pour la suite de l'insertion socioprofessionnelle, une majorité de jeunes semble en emploi trois ans après leur arrêt (Rastoldo et al., 2009) et bon nombre mènent une autre formation à terme. En revanche, la probabilité d'achever une nouvelle formation diminue avec le temps. C'est donc une insertion précaire dans l'emploi qui risque de sanctionner à terme un arrêt prématuré de formation.

42 Cette revue de la littérature portait exclusivement sur la situation (et donc la littérature) suisse. Il faut cependant relever que les travaux portant sur les ruptures d'apprentissage menés en France vont dans le même sens, en ce qui concerne les raisons de l'arrêt, le vécu de celui-ci ou encore l'impact sur la suite du parcours (Capdevielle-Mougnibas, 2009; Cart, Ducourant, Henguelle, Surelle et Toutin, 2007). 
Par ailleurs, les facteurs sociaux évoqués ci-dessus interviennent également dans le parcours post-arrêt. Ainsi, les chances de poursuivre un apprentissage ou de commencer une nouvelle formation sont plus minces chez les jeunes issu.e.s de milieux sociaux défavorisés (Rastoldo et al., 2009), chez les jeunes d'origine étrangère (Rastoldo et al., 2009; Stalder et Schmid, 2006a), chez celles et ceux ayant un faible capital scolaire ou ayant suivi une formation élémentaire (Rastoldo et al., 2009; Stalder et Schmid, 2006a) et enfin, chez les jeunes dont le parcours antérieur est non linéaire. Cette probabilité plus faible de réintégrer une formation est due au fait qu'elles ou ils ont déjà subi un échec (scolaire ou professionnel) avant d'interrompre leur apprentissage (période sans formation ou interruption d'une formation du cycle secondaire II) (Stalder et Schmid, 2006a). Lorsque plusieurs de ces facteurs apparaissent conjointement, on peut véritablement parler de cumul de désavantages. Face à l'arrêt prématuré, il n'y a pas d'égalité entre les apprenti.e.s, un certain nombre de variables sociodémographiques intervenant dans le déroulement du processus. L'arrêt de formation témoigne donc d'un phénomène plus global d'inégalités entre celles et ceux qui peuvent et celles et ceux qui ne peuvent pas accéder à l'emploi ? (Nicole-Drancourt et Roulleau-Berger, 2002).

Enfin, si la variable sexe n'a pas permis de montrer des écarts significatifs entre filles et garçons dans les taux de résiliation de contrats (l'écart en défaveur des garçons est minime: Stalder et Schmid, 2006; Rastoldo et al., 2009), il faut cependant relever un paradoxe: les filles ont globalement des résultats supérieurs à ceux des garçons au sortir du secondaire (Couppié et Epiphane, 2004), pourtant elles font des choix moins prestigieux et se retrouvent plus fréquemment dans des solutions transitoires (Meyer, 2004; Rastoldo, Evrard et Amos, 2007). En outre, il s'agit de mettre l'accent sur un autre élément, qui renvoie directement à la question des choix moins prestigieux. Après un arrêt, les filles optent fréquemment vers une formation supérieure à celle qu'elles ont quitté, pendant que les garçons font le mouvement inverse. Enfin, les pionnières connaissent des parcours plus difficiles et de ce fait, un taux de rupture plus élevé. 


\section{Chapitre 3 \\ Soumettre la formation professionnelle à une analyse de genre}

Sans faire l'histoire du passage des études femmes (Women Studies) aux premiers travaux en études féministes et aux études genre (Delphy, 1998; Parini, 2006; Roux, 2000), il faut rappeler ici que de nombreuses recherches, notamment dans le domaine de l'éducation, ont d'abord mis l'accent sur l'exclusion des femmes, notamment des filières de formation supérieures, avant de s'attacher au rattrapage du «retard historique» des filles. Ce faisant, ces études se sont focalisées sur les avancées spécifiques à l'un des groupes de sexes (niveau de formation, filières), mais surtout sur les filles. Plus récemment, les recherches, en particulier celles concernant le décrochage scolaire, ont analysé prioritairement les garçons (Collectif, 2003), mettant à nouveau l'accent sur l'un des groupe de sexes. La perspective adoptée ici privilégie les travaux qui ont déplacé la focale sur les rapports de genre, par exemple ceux où est observé ce qui se passe concrètement dans les salles de classe, en termes de production de savoirs et de normes (Baudoux et Noircent, 1997; Bouchard et St-Amant, 1996; Mosconi, 1994).

\subsection{Genre, rapports sociaux de sexe, système de genre: quels concepts pour traduire la réalité de la formation professionnelle?}

\subsubsection{Genre et catégorisation}

Le concept de genre est utilisé ici pour parler de l'ensemble des « dispositifs socioculturels qui définissent le féminin et le masculin" (Parini, 2006: 21). Le terme de genre a permis de «trouver un concept qui puisse rompre avec la focalisation sur la situation des femmes comme groupe social en soi et qui permette de questionner le sexe biologique. La distinction entre sexe et genre a été l'un des moteurs principaux de ce changement en remettant en cause la «naturalité > des rôles sexués et en focalisant l'attention sur les phénomènes de construction sociale du sexe» (Parini, 2006: 14-15). Avec le concept de genre, il ne s'agit plus de penser les femmes et les hommes ou le féminin et le masculin en termes de traduction sociale du sexe, mais en termes de catégories produites 
par des rapports sociaux hiérarchiques. Ainsi, le genre rend compte de l'«organisation sociale de la relation entre les sexes» (Scott, 1988). Cette relation est un rapport de hiérarchie fondé sur les différences perçues entre mâles et femelles et légitimé par celles-ci. Dès les années 70, les recherches féministes se sont accordées «sur le fait que la différence des sexes est utilisée comme principe de division sociale hiérarchique, légitimant la domination des hommes et la subordination des femmes » (Roux, $2000: 2$ ). Le terme de "genre» a alors permis de dénaturaliser le sexe et les catégories de sexe, et d'analyser au contraire leur production sociale dans tous les aspects de la vie quotidienne. Son adoption peut être considérée comme un tournant épistémologique, amenant une série d'interrogations nouvelles.

Travailler sur le genre implique nécessairement une réflexion sur la catégorisation (Hurtig et Pichevin, 2002), un processus central dans l'organisation, la différenciation et la hiérarchisation des groupes de sexe. Ce processus visant à simplifier le réel tend à figer chacune des catégories: les différences entre elles sont accentuées, tandis que les similitudes au sein du même groupe sont renforcées (Hurtig, 1998). Sans cesse utilisées dans l'appréhension de ce qui nous entoure, les catégories de sexe sont déterminantes pour saisir les personnes (qu'il s'agisse d'autrui ou de soi) et pour décoder les échanges sociaux. Ceux-ci sont troublés dès lors qu'il est difficile d'attribuer une personne à un groupe de sexe. A ce propos, West et Zimmermann (2009) donnent un exemple particulièrement probant: il s'agit d'une interaction entre une cliente et une personne travaillant dans un magasin d'informatique, dont la cliente ne parvient pas à définir le sexe de manière indiscutable. Dans leur échange, la cliente n'aura de cesse de chercher et trouver des indices (taille des mains, tonalité de la voix, présence/absence de seins ou d'une pomme d'Adam) qui lui permettraient de déterminer le sexe de la personne avec laquelle elle interagit. Elle ne peut se résoudre à entrer en relation en ignorant cette information « essentielle». Pourtant, aucun des indices ne sera entièrement fiable. Cet exemple illustre la prégnance de la catégorisation de sexe dans l'appréhension quotidienne d'autrui et dans la communication, mais il souligne aussi l'arbitraire et la non pertinence des «indicateurs» des prétendues différences entre femmes et hommes.

Un certain nombre de problèmes spécifiques se pose dans le processus de catégorisation de sexe. D’abord, les groupes sont construits sur la base de différences biologiques, instituées comme pertinentes. Cela participe à l'élaboration d'un «consensus social quant à une dualité fondamentale des sexes, [...] dualité [qui] s'appuierait sur une différence 
naturelle, la bi-catégorisation d'ordre biologique impliquant la complémentarité des rôles sociaux» (Zaidman, 2007: 77-78). Ensuite, les catégories de sexe sont construites de manière dichotomique et exclusive (on est homme ou femme), et font l'objet de prescriptions très strictes. Ces prescriptions sociales sur ce qu'est ou n'est pas une femme/un homme entraînent le déroulement de tout une chaîne de stéréotypes, dont les personnes sont captives. Les préjugés étant largement partagés, on y est soumis combien même on ne s'y reconnaît pas. Au cœur du système se trouvent les notions de féminité et de masculinité, notions relativement flottantes, mais fortement associées aux deux groupes sociaux, femmes et hommes. Il est donc attendu qu'une femme soit féminine et un homme masculin, même si ce qui est féminin ou masculin peut changer. Dans les sociétés occidentales contemporaines, la féminité est généralement associée à «l'expressivité et la communialité - c'est-à-dire l'aptitude à l'expression émotive, à l'ouverture à autrui, à la disponibilité relationnelle» (Hurtig, 1998: 108), alors qu'à la masculinité est associée «l'instrumentalité ou l'agenticité - c'est-à-dire l'ensemble des qualités d'un sujet acteur de sa propre vie et engagé dans l'action, la production, la transformation» (Hurtig, 1998: 108). Ces «traits» traversent tous les domaines du social et notamment le domaine professionnel, où ils apparaissent comme des compétences reconnues et rémunérées (pour les traits associés au masculin) ou comme des qualités naturelles, ignorées et donc gratuites (pour les traits associés au féminin).

Enfin, le processus de catégorisation ne permet pas seulement de différencier, mais aussi de hiérarchiser les groupes. La dimension asymétrique établie entre les groupes marque la perception des catégories: les membres du groupe dominant sont plus facilement perçus comme des individus, tandis que les membres du groupe dominé tendent à être assimilés à leur groupe (Hurtig, 1998; Hurtig et Pichevin, 2002). En outre, elle trouve une traduction directe dans la division du travail: ce qui est associé au groupe dominant est valorisé, tandis que ce qui est assigné au groupe dominé est dévalorisé.

\subsubsection{La perspective de genre, une analyse transversale des rapports sociaux}

Mettant l'accent sur les relations socialement construites entre le groupe des hommes et le groupe des femmes, la notion de « genre » est utilisée ici indifféremment de celle de "rapports sociaux de sexe » (D. Kergoat, 2000) ou de «système de genre» (Delphy, 1998; Guillaumin, 1992). Toutes ces notions expriment l'idée d'un rapport social, compris comme un rapport 
de pouvoir et de domination qui lie les catégories de sexe: «Ce qui est important dans la notion de rapport social - défini par l'antagonisme entre groupes sociaux - c'est la dynamique qu'elle réintroduit puisque cela revient à mettre la contradiction, l'antagonisme entre groupes sociaux au centre de l'analyse, et qu'il s'agit bien d'une contradiction vivante, perpétuellement en voie de modification, de re-création " (Kergoat citée par Zaidman, $2007: 74$, note 2). Cette compréhension dynamique permet de dépasser une vision qui lierait le rapport de domination à la simple appartenance à un groupe de sexes, il s'agit en effet d'analyser ce qui amène chacune des catégories à tenir la position dans laquelle elle est par rapport à l'autre. Dès lors, la question est «Comment les hommes biologiques sont-ils socialement produits pour être des dominants? Comment cette domination se reproduit-elle au sein de leur catégorie de sexe? Quels en sont les vecteurs, les moyens?» (Daune-Richard et Devreux, 1992:24). Dans cette perspective, le rapport social est en permanence construit par les personnes qui y sont impliquées, elles agissent sur lui en même temps qu'elles sont produites par lui.

Une perspective de genre implique d'analyser les rapports de genre, au sein de la formation professionnelle, de façon transversale: « au niveau du cadre social dans lequel se situent les interactions (approche macrosociale), au niveau des interactions entre individus ou groupes sociaux (approche interactionniste) ou encore au niveau des constructions identitaires des individus (approche individuelle ou psychologique)» (Parini, 2006: 35). Cette approche transversale permet de considérer le social comme incluant «tous les aspects de la vie sociale, depuis les inégalités structurelles jusqu'à l'interaction quotidienne. Elle concerne aussi bien les significations de notre culture au sens large qu'elle informe notre vie sociale quotidienne » (Jackson, 2009: 18). Pour appréhender ces divers niveaux, cette recherche se nour rit d'approches complémentaires. Bien que privilégiant une analyse structurelle de la division sexuelle du travail, le courant interactionniste, qui analyse le genre comme une production sociale sans cesse renouvelée dans les interactions quotidiennes, est également convoqué. En effet, le genre se fabrique (au sens de «doing gender», notion que j'emprunte à West et Zimmerman, 2009) aussi bien dans l'organisation du travail (dans la distribution des tâches par exemple) que dans les interactions directes entre professionnel.le.s et apprenti.e.s. Cela est particulièrement marqué auprès d'une population adolescente pour qui la dimension relationnelle est centrale (dans le maintien ou l'abandon d'une formation, mais aussi dans la construction de l'identité, notamment professionnelle au travers de la relation 
à autrui). Ce livre cherchera à montrer comment se construisent, dans les situations concrètes du quotidien de la formation professionnelle, les rapports sociaux de sexe.

\subsubsection{Au cœur du système de genre: la division sexuelle du travail}

Pour la présente analyse, il est central d'articuler l'ordre social sexué (le genre, les rapports entre femmes et hommes) et la division sexuelle du travail. En effet, «les rapports sociaux de sexe et la division sexuelle du travail sont deux termes indissociables et qui forment épistémologiquement système; la division sexuelle du travail a le statut d'enjeu des rapports sociaux de sexe» (D. Kergoat, 2000:39-40). Il semble en outre nécessaire de faire le lien entre les effets du système de genre sur les ruptures d'apprentissage et la division sexuelle du travail, parce que celle-ci organise non seulement le travail entre apprenti.e.s, mais aussi la logique hiérarchique qui les lient à leurs collègues. L'objectif est donc de «mettrel'accent sur les dimensions matérielles del'oppression, c'est-à-dire pour l'essentiel sur le travail. Les conflits autour du travail (sous-payé ou fourni gratuitement) expliquent pour l'essentiel pourquoi l'antagonisme est au cœur des rapports sociaux de sexe» (Pfefferkorn, 2007: 11-12).

La division sexuelle du travail est une «forme de division sociale du travail [qui] a deux principes organisateurs : le principe de séparation (il y a des travaux d'hommes et des travaux de femmes) et le principe hiérarchique (un travail d'homme "vaut » plus qu'un travail de femme) » (D. Kergoat, 2000: 36). Dans cette recherche, la division sexuelle du travail est une notion centrale, car l'assignation à des tâches (soit la hiérarchie) est à la base de la division en deux catégories de sexe. Pour aller dans le même sens que Delphy (2001), le principe de hiérarchisation est antérieur au principe de division: "le masculin et le féminin sont les créations culturelles d'une société fondée, entre autres hiérarchies, sur une hiérarchie de genre. Ceci ne signifie pas seulement qu'ils sont liés l'un à l'autre, dans le rapport de complémentarité et d'opposition que nous leur connaissons, mais aussi que cette structure détermine le contenu de chacune de ces catégories, et pas seulement leur rapport» (Delphy, 2001 : 256-257). Ainsi, c'est la hiérarchie qui définit le féminin et/ou le masculin et qui se matérialise dans une division des tâches, différenciées selon le sexe. Une partie importante de l'analyse s'inscrit dans cette posture matérialiste (voir en particulier le Chapitre 8) : c'est donc la position qu'occupent les apprenti.e.s dans le rapport social (la hiérarchie de l'entreprise notamment) qui définit les tâches qui leur sont 
assignées. Ces tâches sont elles-mêmes connotées comme féminines, et dévalorisées, ou masculines, et valorisées.

Enfin, la division sexuelle du travail traverse toute l'expérience sociale du travail, qu'il soit domestique ou salarié. En décloisonnant les frontières entre monde du travail et sphère « hors travail», les recherches féministes ont défini «deux lieux, celui de la production (l'activité professionnelle) et celui de la reproduction (la sphère domestique) comme des lieux de travail qui placent les hommes et les femmes dans des rapports sociaux inégalitaires " (Maruani, 2006:45). Le concept de division sexuelle du travail propose alors une approche critique de la répartition des activités. Le travail ne se distribue pas de façon neutre, et si « hommes et femmes ne sont pas en situation d'égalité professionnelle, c'est aussi parce qu'il y a une division inégalitaire des tâches dans la famille» (Maruani, 2006:46). Et si la division inégalitaire des tâches domestiques se répercute sur le travail salarié, l'inverse vaut également. Ce qui est appris dans la sphère professionnelle (ou dans une autre sphère hors famille, comme l'armée, voir à ce propos Devreux, 1992) enseigne aussi comment le travail domestique doit se répartir. Ce lien entre division du travail domestique et division du travail professionnel permet également d'expliquer la construction sociale des qualifications, soit un ensemble d'éléments reconnus pour les hommes et peu, voire non reconnus pour les femmes. Aux uns, les qualifications acquises dans le monde professionnel, valorisées et rémunérées; aux autres, les compétences acquises dans la sphère domestique, naturalisées et non rémunérées.

Bien qu'ayant soulignél'usage indifférencié, dans cet ouvrage, des termes de genre, rapports sociaux de sexe, système de genre, il s'agit de souligner ici la centralité de la notion de rapports sociaux de sexe dans l'appréhension de l'objet étudié et l'élaboration de l'analyse. En effet, bien que le genre rende compte du rapport socialement construit entre les sexes, la notion de rapports sociaux de sexe permet d'appréhender les deux groupes de sexe, et surtout la matérialité du rapport qui les organise entre eux. La division sexuelle du travail permet de saisir ce rapport, en ce qu'elle donne à voir, au travers de l'assignation différenciée des un·e·s et des autres à des tâches données, la construction permanente d'un groupe par rapport à l'autre. Grâce à cette notion, les rapports sociaux s'incarnent dans le travail. Cela permet de faire voler en éclat la conception de catégories de sexes composées d'individus nécessairement du même sexe. En effet, les rapports sociaux de sexe et la division sexuelle du travail qui les organise, permettent de saisir concrètement l'antériorité de la hiérarchisation. L'analyse proposée ici se fondera donc principalement 
sur l'analyse de la division sexuelle du travail pour d'une part, penser les apprenti.e.s comme groupe dominé (et ce indépendamment de leur sexe) et d'autre part, envisager que le statut apprenti peut provisoiremet faire opérer, à certains d'entre eux (les garçons en l'occurrence) une mobilité de genre. L’appréhension de groupes de sexe organisés entre eux en système rend cette analyse possible.

\subsubsection{Deux systèmes étroitement imbriqués: le genre et I'hétéronormativité}

Par rapport à d'autres groupes sociaux hiérarchisés les uns par rapport aux autres, les catégories de sexe ont un statut tout à fait particulier du fait qu'une certaine proximité les lie. Les femmes et les hommes sont unis par des relations de famille (couple, fratrie, filiation) socialement encouragées, voire présentées comme nécessaires. Or, la "proximité [entre les sexes] renforce en permanence la mise en scène d'une différence hiérarchisée» (Zaidman, 2007: 103), laquelle se construit autour du «rituel de complémentarité» (Goffman, 2002: 59). L'idéologie de la complémentarité permet de faire tenir «l'arrangement entre les sexes», chacun e considérant que les deux sexes sont différents, mais nécessaires l'un à l'autre et surtout que leur place respective est juste, immuable et naturelle (Goffman, 2002). L'évidence avec laquelle sont présentées les relations entre les sexes s'inscrit dans le modèle hétérosexuel. Ce système (l'hétéronormativité) est fondé sur l'idéologie de la complémentarité entre les sexes, c'est pourquoi il tend à gommer le rapport antagoniste. La tension qui existe du fait d'un double système d'une part de genre (rapport hiérarchique entre les sexes) et d'autre part, hétéronormatif (complémentarité entre les sexes) engendre un type de relations bien particulières entre les groupes de sexe, entre ségrégation et indifférenciation, par lequel - pour reprendre les termes de Goffman - les femmes et les hommes sont "with-then-appart», ensemble et séparé (Goffman cité par Zaidman, 2007: 103).

Sans entrer plus avant dans des réflexions théoriques sur l'hétéronormativité, ni analyser les travaux intégrant une perspective queer, il s'agit ici de souligner l'articulation entre le système de genre et le système hétérosexuel, l'un ne pouvant pas fonctionner sans l'autre (Jackson, 2009). Ces systèmes interagissent et la question de la sexualité (en particulier hétérosexuelle) intervient dans tous les domaines du social, y compris au travail. Cette norme régit donc également, directement ou indirectement, les rapports sociaux entre les femmes et les hommes dans le champ professionnel. Ainsi, la «permanente sexualisation du travail 
des femmes - l'étalage qu'on fait d'elles, les particularités des codes d'habillement [...] - [est $]^{43}$ loin d'être quelque chose d'accessoire. Cette 〈hétérosexualisation ? [est] codée à l'intérieur de la division genrée du travail: [c'est] un aspect caché des «spécifications personnelles ` requises pour certains emplois, des pratiques quotidiennes de recrutement et de la discipline au travail.» (Jackson, 2009:23). Intégrer cette perspective à la réflexion permet d'analyser certaines situations paradoxales du monde du travail, où les groupes de sexe sont dans un rapport particulièrement antagoniste - surtout dans des situations de transgression de genre, lorsque des femmes pénètrent des secteurs traditionnellement masculins - et parallèlement «jouent» la complémentarité et la connivence (voir Chapitre 8). L'enjeu ici est de montrer comment les apprenti.e.s connaissent durant leur expérience en formation professionnelle une socialisation professionnelle, de genre, mais aussi et en lien étroit à l'apprentissage des normes de genre, une socialisation à l'hétéronormativité.

\subsection{De l'école obligatoire à la formation professionnelle : socialisations et orientations}

La formation professionnelle se situant à l'intersection entre éducation et monde du travail, l'analyser revient à croiser différentes thématiques. Partie intégrante du processus de transition entre l'école et le marché du travail, la formation professionnelle ne peut pas se comprendre sans observer ce qui s'est passé en amont, durant les années de scolarité (secondaire I), au moment de l'orientation scolaire et professionnelle, mais également ce qui se passe en aval, sur le marché de l'emploi. Le système dual est en effet directement lié au monde du travail et une part importante des enjeux y sont similaires (voir Chapitre $1^{\text {er }}$ ).

Ce constat implique, dans une perspective de genre, d'emprunter des réflexions issues de différents champs disciplinaires. Les premières ressources proviennent des sciences de l'éducation et de la sociologie de l'éducation (Chaponnière et Chaponnière, 2006; Duru-Bellat, 1994, 1995b; Mosconi, 1994), en particulier les recherches sur la socialisation différenciée des sexes (Dafflon-Novelle, 2006) et celles sur le curriculum caché etl'effet Pygmalion ${ }^{44}$ observés dans divers types de formation (Bau-

43 Le passage au présent a été fait par l'autrice.

44 On parle d'effet Pygmalion lorsque les élèves se conforment aux attentes des enseignant·e.s, notamment en matière de goût ou de réticence face à une matière enseignée. Mais Mosconi (1994) utilise cette notion avec précaution, car elle laisse penser que les élèves subiraient passivement les effets de l'enseignement. 
doux et Noircent, 1997). Les travaux relatifs aux choix professionnels ou à l'orientation (Baudelot et Establet, 1992b, 1992c; Couppié et Epiphane, 2004 ; Vouillot, 2007), s'imposent également. Il faut bien sûr, pour saisir l'entier des enjeux, y inclure ceux qui traitent des choix atypiques ou pionniers (Croisier, 2002; Dallera et Ducret, 2004; Guichard-Claudic, Kergoat et Vilbrod, 2008; Lemarchant, 2008; Thiébaud, 2004). Ces recherches mettent en évidence un même paradoxe: des filles possédant un capital scolaire élevé et réussissant mieux, en moyenne, que leurs pairs masculins, mais qui continuent à faire des choix peu valorisants en termes de carrière et de rémunération. Ce paradoxe se retrouve pour la formation professionnelle sous une forme à peine modifiée: des filles qui réussissent mieux à l'école secondaire mais qui connaissent tout de même une transition plus délicate vers le marché des places d'apprentissage (voir Chapitre ${ }^{\mathrm{er}}$ ).

L'inscription de la formation professionnelle duale dans un marché du travail fortement ségrégué selon le sexe nécessite également de recourir aux apports des sociologues du travail féministes (voir Chapitre $\left.1^{\mathrm{er}}\right)$. Leurs recherches sont essentielles pour saisir les processus de genre en jeu dans la ségrégation du marché du travail, la concentration des femmes dans certains secteurs d'activité, ainsi que la division sexuelle du travail (D. Kergoat, 2000; Marry, 2006; Maruani, 2006; Maruani et Nicole, 1987; G. Moreau, 2000).

Enfin, l'analyse de la souffrance en formation professionnelle et des stratégies pour y faire face motive le recours à la psychodynamique du travail (Dejours, 1998; Maranda et Leclerc, 2000; Molinier, 2004), en particulier aux études mettant l'accent sur les questions de virilité/ muliérité (au travail) (Molinier, 2000, 2002b) ${ }^{45}$.

\subsubsection{Scolarité et socialisation différenciée des sexes}

Travailler sur le genre en formation professionnelle nécessite de rappeler ce qui a été produit en matière d'inégalités entre les sexes par l'institution scolaire, que ce soit dans l'enseignement ou dans la manière dont les élèves s'orientent ou sont orienté.e.s vers telle ou telle filière.

45 Les réflexions théoriques portant sur la psychodynamique du travail ne seront pas développées ici, car elles ne sont pas transversales à l'entier de la démarche. Elles feront l'objet de parties spécifiques dans les deux chapitres concernés par cette approche, les Chapitres 6 et 7. 


\section{Sociologie de l'éducation et genre}

Bien que la tradition francophone de sociologie se soit penchée depuis longtemps sur les inégalités sociales à l'école, ce n'est qu'à partir des années 90 qu'elle s'est «intéressé[e] à l'impact de la variable sexe sur les trajectoires scolaires» (Duru-Bellat, 1994: 113). Malgré les différences de scolarité entre filles et garçons, perceptibles grâce aux statistiques, elle n'a pas proposé d'analyses spécifiques, y voyant peut-être les restes d'un anachronisme qui tendrait à disparaître avec le temps (Duru-Bellat, 1994, 1995b). De fait, la question de la hiérarchie des sexes a été avant tout introduite dans le champ sociologique par des femmes, dont les travaux ont été réalisés dans un contexte militant féministe. Elles ont misl'accent sur l'accès des filles à l'éducation, notamment dans des travaux historiques, qui soulignent le « retard » en matière de formation des filles, leur accès tardif au baccalauréat et aux études supérieures (Fraisse et Perrot, 1991; Renevey-Fry, 1997) ${ }^{46}$. A la suite de ces premiers travaux, il faut signaler d'autres recherches qui marquent un tournant méthodologique majeur, passant de la macrosociologie et de l'analyse des systèmes à une microsociologie qui s'intéresse à ce qui se déroule en classe.

Autre tournant, l'ouvrage de Baudelot et Establet (1992a), dans lequel ils soulignent que la corrélation entre réussite scolaire et sexe est beaucoup plus pertinente que la corrélation entre réussite scolaire et classe sociale. Ils démontrent aussi qu'à l'issue de la scolarité, les filles ont accumulé davantage de capital scolaire, mais que les garçons négocient mieux le capital qu'ils ont acquis. Pour ces auteurs, les filles seraient nettement désavantagées par l'orientation professionnelle, perdant à cette occasion les avantages acquis.

Les recherches s'intéressent dès lors aux performances scolaires des filles et des garçons, à tout ce que l'institution scolaire transmet en termes de normes durant le cursus (curriculum caché), à la socialisation différenciée (familiale, mais aussi scolaire), et enfin à la question de l'orientation.

46 Il n'est pas pertinent de s'arrêter ici sur la situation actuelle des femmes dans l'éducation, car cela est trop éloigné du propos. Par rapport aux premiers travaux sur le « retard historique», on peut dire que les filles sont aujourd'hui dotées d'un capital scolaire général légèrement supérieur aux garçons, ce qui est inédit. Les filles devancent les garçons dans l'obtention de diplômes à l'issue du secondaire et elles sont $54 \%$ dans les pays membres de l'OCDE à entamer une formation tertiaire. Cependant, si elles entament également des études dans des filières scientifiques (dont elles représentent $30 \%$ des effectifs), elles sont surreprésentées dans les domaines de la santé et du social (75\% des effectifs) (OCDE, 2008). 


\section{Performances scolaires et socialisation}

Les travaux qui mesurent les effets du genre sur la scolarité abordent divers aspects: la performance, l'orientation, les aptitudes ou encore les attitudes face au savoir. Concernant les performances des filles et des garçons en classe, les premières ont de meilleurs résultats. Mais cet «avantage » ne se vérifie que jusqu'à la fin du secondaire: «à partir de la fin du collège ou du début du lycée, et dans les cursus indifférenciés, les écarts se creusent avec une meilleure réussite des filles dans les matières 〈littéraires 〉, et des performances plus centrées autour de la moyenne (voire moins bonnes) dans les disciplines scientifiques» (Duru-Bellat, 1994: 120). Est-ce dû à des différences d'aptitudes ou est-ce le résultat «d'une fabrication familiale ou scolaire»? (Duru-Bellat, 1994: 121). La thèse de la socialisation, dont les effets portent tant sur l'attitude face aux apprentissages que sur le savoir en général, est bien évidemment retenue ici. Par socialisation, il ne faut pas comprendre «un processus mécanique de confrontation à des «modèles exemplaires de comportement social approprié aux deux sexes> (Maccoby, 1990), mais [...] [la manière dont] l'enfant garçon ou fille apprend des choses différentes via ses expériences quotidiennes dont on sait qu'elles sont largement différenciées dès sa naissance» (Duru-Bellat, 1994: 124). Cette socialisation est composée non seulement d'attentes parentales différenciées en termes de «carrières » selon le sexe de l'enfant, mais encore de différences éducatives prodiguées au sein de la famille, autant d'éléments qui influent sur le rapport au savoir, sur l'autonomie, la confiance en soi et l'estime de soi. Ces apprentissages sont en outre confortés par ceux faits dans l'institution scolaire.

\section{Le curriculum caché}

Un pan important des travaux sur l'impact du genre dans les parcours d'éducation s'intéresse à la construction scolaire des différences entre les sexes. Ce sont les recherches de microsociologie observant ce qui se passe dans les salles de classe en matière de production et de transmission de normes de genre. Elles se sont penchées sur les interactions entre maîtres-ses et élèves comme sur celles entre élèves. Dans la même optique, elles ont analysé le matériel pédagogique utilisé et les programmes officiels. Ces études constatent le recours fréquent à l'opposition filles-garçons dans la gestion de la classe, une attention plus grande apportée aux garçons (Baudoux et Noircent, 1997; Duru-Bellat, 1995a; Mosconi, 1994; Solar, 1993), que ce soit en nombre d'interactions (la règle des 
«deux-tiers» $)^{47}$ ou dans la qualité de celles-ci. Les garçons sont ainsi davantage sollicités, prennent plus la parole et sont davantage corrigés, évalués, repris. Ils sont considérés comme des individus, pendant que les filles sont perçues comme un groupe au sein duquel elles ont moins le statut de sujet distinct que les garçons. Ces mêmes travaux attestent que les enseignant.e.s attendent de leurs élèves une conformité à des normes de genre (en termes d'attitudes, de positions dans les rapports sociaux de sexe, par exemple). Le jugement des enseignant.e.s (remarques, mais aussi notes) valide donc avant tout l'adéquation des élèves à la norme: les travaux sont complimentés pour la réflexion chez les garçons, pendant qu'on remarque la propreté et l'écriture chez les filles...

Ces études s'intéressent également aux relations entre élèves et montrent que les jeunes ne subissent pas seulement des attentes de conformité à des normes et des discriminations, mais qu'elles et ils y participent. Il n'y a donc, de la part des élèves, pas d'adaptation passive ou de soumission à des stéréotypes, "car dans la classe, les élèves apprennent activement leur rôle de sexe» (Duru-Bellat, 1995b: 82). En d'autres termes, certaines attitudes participent de la construction de l'identité de genre: jeux de virilité, refus de l'autorité scolaire, et jeux de féminité, peur de certaines disciplines ou de certaines manipulations (en sciences), indiquant surtout aux autres que l'on est bien conforme aux normes. Dans le quotidien de la classe, le genre se donne à voir (au sens de "doing gender", West et Zimmermann, 2009), se met en scène dans les interactions entre élèves comme entre élèves et enseignant.e.s. Ainsi, les enseignant.e.s qui tentent de rééquilibrer le temps consacré aux élèves des deux sexes doivent affronter des résistances de la part des garçons. La socialisation scolaire est donc le résultat d'une interaction entre des élèves socialisé.e.s auparavant dans le cadre de leur famille et des enseignant.e.s qui répondent aux différences de comportements des élèves par des attentes le plus souvent stéréotypées.

A un niveau plus global, il faut encore signaler le contenu des programmes, qui reste largement problématique puisque décliné au masculin (auteurs, philosophes, approches de l'histoire, etc.), ainsi que l'institution scolaire et ce qu'elle montre en termes de ségrégation (enseignantes surreprésentées au pré-scolaire et dans les petites classes, enseignants de plus en plus nombreux dans les niveaux les plus élevés

47 Lors des échanges menés dans les classes, deux-tiers des interactions ont lieu entre l'enseignant·e et les garçons, alors que les filles ne bénéficient que d'un tiers de l'attention. Cela se révèle tant lorsque la parole est distribuée parl'enseignant.e que lorsque les élèves prennent la parole «spontanément». 
et à l'université). Cette ségrégation est particulièrement marquée dans le champ de la formation professionnelle", où le taux de féminisation de l'enseignement est d'environ 30\% (OFS, 2013).

La transmission de toute cette connaissance implicite des rapports sociaux, connaissance que certain.e.s nomment le curriculum caché, joue un rôle non négligeable sur les apprentissages: prise de parole, mise en retrait, confiance en soi, estime de soi. Ainsi, bien qu'ayant un capital scolaire plus élevé, les filles sortent de l'école avec une faible estime de soi (Baudoux et Noircent, 1997; Duru-Bellat, 1994; Mosconi, 1994 ; Solar, 1993). Ce paradoxe influe sur les choix d'orientation et les parcours ultérieurs.

Que ce soit en classe, avec le curriculum caché, ou dans les autres lieux de socialisation (famille, activités de loisirs, sport), les enfants apprennent la conformité, ce qui signifie pour les filles l'apprentissage du renoncement (Mosconi, 1994) ou encore l'évitement de situations de compétition. Ces attitudes visent notamment à garder la place qui est la leur dans la hiérarchie entre les sexes. Nombre de situations sont l'occasion d'affirmer sa conformité à la norme : c'est le cas non seulement dans le choix des filières, mais aussi dans le succès au sein de celles-ci. Les filles réussissent mieux dans les disciplines connotées féminines et les garçons dans celles identifiées comme masculines. Par ailleurs, ils ont de meilleurs résultats en lecture, lorsque la discipline n'a pas de connotation féminine. Quant à l'orientation, elle résulte des divers processus de socialisation vus précédemment. Les filles s'excluent des filières scientifiques, ce qui leur permet d'affirmer leur conformité aux normes de la féminité (Vouillot, 2007).

\subsubsection{Le choix d'une profession: entre choix par défaut et orientation au service du genre}

Un second paradoxe s'ajoute au précédent: malgré une bonne réussite scolaire, les filles font fréquemment le choix de filières professionnelles peu prestigieuses (Couppié et Epiphane, 2004). Comme cela a été mentionné pour le secondaire, ily a un phénomène d'auto-sélection, les jeunes femmes se considèrent, à notes égales, moins bonnes que les garçons et demandent moins souvent l'accès à des filières prestigieuses ou offrant de bons débouchés (Marry, 2004a). Différentes pistes explicatives sont proposées dans la littérature, elles seront examinées selon quatre catégories d'analyse, dont trois sont développées par Marry (2004a) : l'acteur ou l'actrice dupé.e, l'agent.e stratège, l'acteur ou l'actrice mobilisé.e, et la quatrième inspirée par Vouillot (2007): le jeu d'acteur ou d'actrice. 


\section{Choix dupé}

Baudelot et Establet (1992c) expliquent le paradoxe d'une meilleure réussite des filles et de leur auto-sélection, voire de leur élimination des filières d'excellence par une approche fondée sur la théorie de l'habitus (Bourdieu, 1984). Tenant compte de la socialisation différenciée, les auteurs considèrent les parcours de formation des filles comme des "choix de dupe» (Couppié et Epiphane, 2004). Les filles présenteraient un habitus sexué de soumission et de docilité qui leur permettrait de mieux répondre aux attentes du système scolaire que leurs homologues masculins, tout en restant cantonnées dans des filières «féminines » et dans certains métiers. Dans cette perspective, elles apprendraient « dès la prime enfance, [...] la docilité, l'attention à autrui, la persévérance dans la tâche, l'usage limité de l'espace; [pendant qu'] eux apprennent la compétition, l'affirmation du moi, l'usage somptuaire de l'espace. Elles sont donc mieux adaptées aux réquisits de l'école» (Marry, 2004a : 34). Conséquence de cet habitus : les filles se sous-évaluent et s'auto-éliminent des filières scientifiques, confortées dans ce « choix » par les enseignant.e.s. Cette approche s'inscrit dans la droite ligne des théories de la reproduction des inégalités sociales par l'école. Ainsi, «à l'instar des enfants d'ouvriers, les filles feraient des choix de «dominées `. En optant pour des études et des métiers qui prolongent les fonctions traditionnellement dévolues aux femmes dans la famille (enseignement, santé, relations...), elles intérioriseraient leur destin le plus probable, celui d'épouse et de mère, dont le travail professionnel demeure secondaire par rapport à celui de l'homme...» (Marry, 2004a: 34).

\section{Choix raisonné}

L'approche proposée par Marie Duru-Bellat dans l'Ecole des filles (Duru-Bellat, 1990) récuse ces interprétations. Pour elle, les filles ne feraient pas des choix de dupe, mais raisonnés et adaptés à la place qui leur est socialement assignée dans leur future famille et sur le marché du travail. Cette approche fondée sur une théorie de l'acteur (Couppié et Epiphane, 2004) présente les choix comme le résultat de stratégies conscientes et rationnelles, et non comme des décisions liées à une intériorisation de la domination masculine. Les jeunes femmes - et les jeunes hommes - anticiperaient leurs futurs rôles sociaux. Plus particulièrement, les jeunes femmes semblent devoir faire, déjà a priori, des arbitrages entre travail et famille «tant elles se sentent responsables de ces problèmes, et n'imaginent pas de redéfinir le partage des tâches avec leurs futurs compagnons» (Boyer et al., cités par Duru-Bellat, 1995b: 
97); ou encore: « Les filles [...] arbitrent entre des carrières prestigieuses mais prenantes (ces carrières de haut niveau qui supposent souvent des mathématiques...), et des ‘ choix de compromis > pour des professions certes moins valorisées, mais où le temps partiel est possible ou les conditions de travail souples ( (Duru-Bellat, 1995b: 97). Les contraintes sont ainsi largement intériorisées, et les jeunes femmes présentent ces orientations comme de réels choix (Dallera et Lamamra, 2008; Lamamra et Rosende, 2005). Cette stratégie semble d'ailleurs moins coûteuse, psychologiquement et professionnellement. Des choix moins traditionnels peuvent être lourds de conséquences, puisqu'il faut parfois payer le prix de la transgression (Le Feuvre, 2008; Legault, 2001; Marry, 2007).

\section{Actrices mobilisées}

Une troisième approche conteste la lecture des orientations en termes d'anticipation des contraintes ou de soumission aux normes. Elle rappelle la transformation historique des modèles éducatifs, notamment les investissements parentaux dans la réussite scolaire et professionnelle qui sont devenus similaires pour leurs filles comme pour leurs garçons. Cette évolution se vérifie surtout pour les enfants issu.e.s des classes les plus favorisées. Par ailleurs, outre un projet parental de mobilité sociale au travers de la formation, un autre changement historique est à relever: les mères transmettent souvent aujourd'hui des valeurs égalitaires (Marry, 2004a). Ces éléments auraient conduit les filles à un plus grand investissement dans la réussite scolaire. Autres gagnantes du processus, les filles des milieux ouvriers ou immigrés, qui connaissent un niveau de réussite scolaire largement supérieur aux garçons des mêmes catégories sociales (Baudelot et Establet, 1992c). Cette approche infirme par ailleurs la théorie du coût de la transgression, en soulignant, statistiques à l'appui, que dans les filières ou les professions non traditionnelles, les filles connaissent des conditions d'insertion et de carrière supérieures à ce qu'elles auraient trouvé autrement, et ce malgré des conditions qui restent moins favorables que celles faites aux hommes (Couppié et Epiphane, 2008; Le Feuvre, 2008; Marry, 2008).

\section{Jeu d'actrices}

La dernière approche en matière de choix d'orientation et de choix professionnels est celle proposée par Vouillot (2007). Intégrant tant des éléments de la socialisation et de la théorie de la reproduction, que des éléments issus des réflexions en termes de choix raisonnés, elle apporte un élément supplémentaire, en proposant une deuxième interprétation à 
ce qui motive le raisonnement : ce n'est pas uniquement vers l'aval qu'est tourné le choix raisonné, mais également vers le moment présent. L’autrice rappelle que le choix professionnel se déroule durant l'adolescence, moment de construction identitaire, notamment de l'identité sexuée. Ainsi, l'enjeu du choix d'orientation est certes tourné vers les projets professionnels, mais aussi vers le groupe de pair.e.s. Il s'agit, à travers l'orientation choisie, de montrer sa conformité de genre à son entourage. Moment fort del'identification à des stéréotypes (Dafflon-Novelle, 2006), l'adolescence ne s'accommode guère de transgressions de genre. Choisir un métier ou une filière de formation, c'est dire aux autres qu'on est bien une fille féminine ou un garçon masculin. Dans les termes de Vouillot (2007), l'orientation se met alors au service du genre. On peut également y voir une parade de genre (Goffman, 2002), permettant de signaler à son vis-à-vis - ici tout l'entourage social (famille, corps enseignant, psychologues du conseil en orientation) - son adéquation de genre. C'est un bel exemple de fabrication du genre (au sens de "doing gender ", West et Zimmermann, 2009).

\section{Vers une intégration de ces différentes approches}

La perspective adoptée dans ce travail combine ces différentes approches. Impossible de mettre de côté les apports des travaux sur la socialisation et le curriculum caché, et s'il n'est pas entièrement dupé, le choix des jeunes femmes n'en est pas moins le produit de cette socialisation. Pour autant, le choix est renégocié par les actrices, car elles anticipent les assignations de genre, en particulier la division sexuelle du travail. Compte tenu du terrain particulier (la formation professionnelle destinant à des métiers et non à des professions prestigieuses), la troisième approche sera écartée. En effet, l'étape de la formation professionnelle ne permet pas encore de mesurer le " coût de la transgression ». Il est trop tôt dans le parcours professionnel pour savoir si un choix atypique pourra se révéler - pour les jeunes femmes - plus payant qu'un choix traditionnel, et ce malgré les difficultés rencontrées. En outre, une étude sur les arrêts prématurés en formation professionnelle met surtout l'accent sur les importantes difficultés rencontrées, et ce notamment lors d'insertions atypiques. Cette population ne se prête donc pas nécessairement à une réflexion sur les avantages ou les coûts relatifs de la transgression. L'approche proposée par Vouillot (2007) est également intéressante, car non seulement l'orientation, mais également le déroulement de la formation professionnelle et les stratégies utilisées pour s'y maintenir ou en partir sont au service du genre, à un moment où les adolescent.e.s 
doivent à la fois faire des choix en matière de formation et de profession, et en matière d'identité sexuée. Ainsi, le processus qu'elle décrit pour l'orientation se retrouve lors de différentes activités: au travail, lors de réunions, de jeux, d'activités sportives, de conversations, autant de «situations sociales où à travers des rituels et des parades expressives, les hommes et les femmes peuvent effectivement mettre en représentation les différences de nature que la société dit être les leurs» (Goffman cité par Zaidman, 2007 : 106). En élargissant les réflexions de Vouillot (2007) et en les faisant rencontrer celles de West et Zimmermann (2009), le but sera d'identifier les divers moments où se fabrique le genre durant l'expérience de formation professionnelle.

\subsection{La question des pionnières et des pionniers}

Malgré les enjeux définis précédemment autour des questions d'orientation, certaines personnes optent pour un choix atypique, c'est-à-dire qu'elles entrent dans un secteur professionnel où elles sont minoritaires. Sont considérées personnes pionnières, les femmes occupant des emplois traditionnellement masculins et les hommes insérés dans des emplois traditionnellement féminins ${ }^{48}$. Un certain nombre de discussions traversent les travaux sur les parcours pionniers, en particulier le taux à partir duquel on considère qu'une profession est atypique. Bien que certains travaux placent le seuil assez bas, à l'instar de Croisier (2002) qui le met à $12 \%$, a été classée comme pionnière toute profession au sein de laquelle la proportion de l'un ou l'autre sexe est inférieure à $33 \%$. Ce choix s'est fondé sur la démarche adoptée par le Secrétariat à la condition féminine du Gouvernement du Québec ${ }^{49}$, qui a produit depuis des années beaucoup de travaux sur la question et pour lequel un emploi est considéré comme non-traditionnel lorsque les femmes, respectivement les hommes, représentent moins du tiers de la main-d'œuvre totale. Ce taux permet d'englober des professions qui sont en train de devenir mixtes, mais dont la culture ne l'est pas encore (pour les professions pionnières dans le canton de Vaud, voir Annexe 1). Dansla recherche, des «situations" pionnières ont été identifiées. Il s'agit de situations, où des personnes occupent des emplois traditionnels pour leur sexe, mais dans

48 Est qualifiée de «masculine», respectivement de «féminine» une profession non seulement composée majoritairement d'hommes, respectivement de femmes (Couppié et Epiphane, 2008), mais encore - et en lien avec cette sur-représentation numérique - une profession traditionnellement connotée comme masculine, respectivement féminine. 
un environnement où elles sont minoritaires (par exemple les employées de commerce qui travaillent dans un garage $)^{50}$. Bien qu'engagées dans des formations mixtes, ces personnes sont confrontées sur leur lieu de travail à une certaine résistance masculine (Dallera et Ducret, 2004).

Les insertions pionnières signalent à la fois les changements qui sont intervenus sur le marché du travail (les professions se sont ouvertes, notamment aux femmes) et les invariants (bien que les premières insertions atypiques remontent à plusieurs années, on parle toujours de pionnières et de pionniers). Comme le souligne Marry (2007), dans les métiers ouvriers, les pionnières se succèdent indéfiniment. Elles rencontrent une constellation de difficultés : réticences des employeurs, brimades des collègues ou de la clientèle, horaires et conditions de travail difficiles à articuler avec une vie privée, difficultés dans la carrière et enfin difficultés dans la vie de couple. Elles paient le prix fort de la transgression. Parallèlement, il semblerait que l'insertion dans l'emploi diffère selon le niveau de la profession. On constate plus de difficultés pour les professions ouvrières que dans l'ingénierie et les professions techniques, où les femmes accèdent plus rapidement à des emplois plus stables et mieux rémunérés que dans les professions mixtes ou féminisées (Couppié et Epiphane, 2008; Marry, 2007, 2008). La mixité induit donc à la fois des avancées et des résistances.

\subsubsection{Des pionnières pour répondre aux besoins de l'économie}

La question des orientations atypiques doit également être comprise comme une réponse structurelle à des changements intervenus sur le marché du travail. Ainsi, «si l'avancée en mixité est incontestablement le fait de celles qui se sont mobilisées individuellement et collectivement, il n'en reste pas moins que leur arrivée est directement liée aussi à certains facteurs structurels, notamment les décisions des pouvoirs publics de mettre fin aux discriminations vis-à-vis des femmes dans toute une série de professions qui leur étaient interdites, les transformations qui ont marqué certains métiers dans une période récente (par exemple dans l'imprimerie ou le transport routier) ou encore les difficultés de recrutement dans certaines professions. En effet, quand les «vocations > masculines se raréfient, quand les rémunérations sont faibles, quand le turn-over est important, organisations professionnelles et pouvoirs

$50 \quad$ Compte tenu de leur très faible nombre $(\mathrm{N}=2)$, ces personnes ont été analysées avec les pionnières et les pionniers. 
publics n'hésitent pas à se tourner vers les femmes et à promouvoir auprès d'elles des métiers qui leur étaient jusqu'alors fermés »(Pfefferkorn, 2008: 113). La corrélation entre recrutement de femmes et désaffection des hommes de certaines filières techniques et scientifiques se vérifie tant à de hauts niveaux (doctorats) que dans les ateliers (Lemarchant, 2007). Dans cette logique compensatoire, la promotion des femmes explique en partie pourquoi l'avancée en mixité ne signifie pas pour autant la fin de la ségrégation: "Pour l'heure on ne peut donc pas dire que la mixisation débouche sur la substituabilité ou l'indifférenciation des sexes, loin s'en faut: dans les métiers masculins investis par les femmes, les stratégies de virilisation des hommes insistent au contraire sur la différenciation sexuelle» (Guichard-Claudic et al., 2008: 18). En outre, les clivages sexués restent très marqués lorsqu'on prend en compte la position hiérarchique, le statut ou encore les activités précises effectuées dans l'exercice du métier. Au fond, le contenu des emplois atypiques varie selon une division sexuelle du travail traditionnelle. Cette situation met les personnes pionnières dans une position paradoxale: les filles par exemple, qui «ont choisi un métier d'homme pour fuir les métiers féminins répétitifs, sédentaires et la subordination domestique, [sont constamment renvoyées à leur identité de filles] par des mises à l'épreuve (bizutage, défis...) qui visent à démontrer leur infériorité « naturelle ` dans ces métiers ou de façon plus insidieuse, par l'imposition d'un stéréotype féminin, négatif positivé du masculin: ils sont forts, elles sont «minutieuses > (et non pas faibles)» (Marry, $2007: 125)$. Ces stéréotypes sont en contradiction avec la motivation des pionnières, qui adhèrent souvent à une logique d'éloignement des rôles et places qui leur sont assignées traditionnellement. Elles sont donc tiraillées entre leur volonté ou leur parcours de transgression d'un côté et la tendance des collègues à les renvoyer à leur statut de femmes de l'autre.

\subsubsection{Le sexe des parcours pionniers}

De nombreux travaux ont montré que les parcours professionnels atypiques varient fortement en fonction du sexe (Croisier, 2002; Thiébaud, 2004). Les filles qui choisissent d'apprendre un "métier d'hommes" parviennent bien moins souvent que les garçons à obtenir un poste de travail correspondant à leur formation et elles éprouvent plus de difficulté que leurs collègues masculins à mener une carrière professionnelle ascendante (Gallioz, 2008).

Cette asymétrie se manifeste à divers niveaux (Marry, 2007): décision d'entrer dans la profession et type de motivation (choix par 
défaut pour les garçons, choix raisonné pour les filles) (Lemarchant, 2007), âge de prise de cette décision (elle est plus tardive chez les garçons), mécanismes d'entrée, carrière possible une fois inséré.e dans la profession (les garçons parviennent facilement à occuper les postes à responsabilité dans les professions dans lesquelles ils sont pionniers) (Le Feuvre et Laufer, 2008), enfin accueil reçu dans le milieu professionnel (hostilité pour les filles, bienveillance pour les garçons) (Croisier, 2002; Thiébaud, 2004). Quant au prix à payer face à cette transgression, il n'est pas le même et ne se joue pas au même endroit. Les femmes ont à affronter les résistances de leur environnement de travail, les humiliations, le sexisme, la violence (Eckert, 2007; Legault, 2001 ; Marry, 2007), mais peuvent être encouragées par leur entourage, ce type d'insertion s'inscrivant dans un schéma de mobilité ascendante (Daune-Richard et Devreux, 1992; Guichard-Claudic et al., 2008). Pour leur part, les hommes ont à faire face au soupçon d'homosexualité de leur entourage (parents, enseignant.e.s, camarades de classe), mais sont généralement les bienvenus dans leur environnement de travail (Thiébaud, 2004). Cette asymétrie se manifeste également à un niveau identitaire : en raison de la hiérarchie des sexes, le "coût psychologique» d'un choix atypique n'est pas le même pour les garçons que pour les filles. La féminisation des bastions masculins est vécue comme une menace par les hommes (Eckert, 2007; Guichard-Claudic et al., 2008; Maruani et Nicole, 1989), alors que les femmes présentent de manière positive l'avancée en mixité (que ce soit pour leur propre insertion pionnière ou pour celle des hommes) (Guichard-Claudic et al., 2008; Le Feuvre et Laufer, 2008). Enfin, au-delà du coût psychologique, l'impact en termes de trajectoire diffère, l'entrée des filles dans une profession traditionnellement masculine étant perçue comme un parcours de mobilité ascendant ${ }^{51}$, pendant que l'entrée des garçons représente un véritable déclassement. Mais ce processus ne s'arrête pas là, une fois dans le métier atypique, les pionnières et les pionniers sont fréquemment assigné.e.s à des tâches spécifiques en raison de leur sexe, on pourrait alors parler de reclassement ${ }^{52}$. Ces constats montrent

51 L'idée de mobilité de genre est empruntée à Guichard-Claudic (2008). Celleci peut être ascendante ou descendante, c'est dans le prolongement de cette deuxième situation que le terme de déclassement est utilisé.

52 Ces mécanismes de recréation ou de perpétuation de la division sexuelle du travail, y compris lorsque les personnes sont insérées dans des professions non traditionnelles ont été bien montrées par Le Feuvre et Laufer (2008). C'est ce processus de réassignation à sa catégorie de sexe qui est qualifié ici de reclassement. 
que derrière l'avancée en mixité se cachent des enjeux différents selon les secteurs d'activité (dévalorisation ou revalorisation).

Au terme de ces trois premiers chapitres, une image assez sombre de la formation professionnelle sous l'angle de l'égalité entre les sexes se dessine. Il apparaît en outre que la perspective de genre est rarement mobilisée pour analyser ce système de formation ou encore les arrêts prématurés qui s'y déroulent. Enfin, compte tenu de la spécificité de cet espace de formation au carrefour entre école et marché du travail, des emprunts théoriques sont faits tant à la sociologie de l'éducation, aux travaux portant sur les choix d'orientation, qu'à la sociologie du travail.

Dès sa mise en œuvre à la fin du XIX ${ }^{\mathrm{e}}$ siècle, le système suisse de formation professionnelle s'écrit au masculin, inscrivant en son sein les inégalités. Dans les premiers temps, cette filière est en effet destinée prioritairement aux jeunes hommes suisses, participant dans ce domaine de formation comme dans les autres de cette époque à entériner le «retard» des femmes. Au-delà de la mise en place du système, sa particularité, à savoir son imbrication étroite avec le marché du travail, fait qu'il reproduit les inégalités (ou qu'il y prépare). Ainsi, des phénomènes typiques du marché du travail tels que les diverses formes de ségrégations, les discriminations, la concentration ou encore la division sexuelle du travail peuvent s'observer en formation professionnelle. Ce lien étroit entre marché du travail et système de formation fait que les changements intervenus dans le premier ont des répercussions sur le second, et les pressions accrues sur les femmes se retrouvent ainsi dès l'apprentissage. Cela explique pourquoi de nombreux outils théoriques sont empruntés à la sociologie du travail féministe. Enjeu très débattu dans la littérature suisse, la question de la transition école-travail, qui accompagne toute réflexion surl'apprentissage, notamment dual, est également interrogée à l'aune du genre. Il s'avère que là encore, les inégalités transparaissent, et que les jeunes femmes, malgré un capital scolaire tendanciellement plus élevé, abordent cette étape avec plus de difficultés. Ce premier regard sur le système suisse permet d'affirmer qu'il ne reproduit pas uniquement des logiques construites en amont (durant la scolarité obligatoire) ou en aval (sur le marché du travail), mais qu'il est bien un espace avec ses logiques propres, y compris en termes de production d'inégalités.

L'état de la littérature suisse sur le phénomène d'arrêts prématurés en formation professionnelle a mis l'accent sur la quasi absence d'analyse 
de genre. Les travaux ont rarement exploré les parcours particuliers des un.e.s et des autres, créant l'illusion d'un phénomène touchant également filles et garçons, alors que les jeunes ne sont pas inséré.e.s dans les mêmes secteurs professionnels et ne connaissent donc pas les mêmes situations de formation ou de travail. Ils n'ont pas non plus cherché à questionner un paradoxe qui s'ajoute à ceux déjà connu de la littérature (capital scolaire élevé, mais transition ou insertion professionnelle difficile), à savoir une transition globalement difficile pour les filles, mais une moindre proportion d'entre elles touchées par les arrêts prématurés.

Enfin, la partie théorique a permis de revenir sur le concept de genre comme outil d'analyse transversal. Elle a tout particulièrement mis l'accent sur la notion de division sexuelle du travail centrale à l'analyse proposée dans cet ouvrage. Dans ce chapitre, la spécificité du système dual est à nouveau au centre du propos. En effet, pour analyser cet espace particulier, des emprunts à divers cadres sont nécessaires. En effet, s'inscrivant dans la poursuite du système éducatif, la formation professionnelle hérite de ce que l'école a produit en termes de socialisation et de curriculum caché. En outre, la formation professionnelle fait également suite au processus de choix et d'orientation professionnelle, aussi ne peut-on comprendre ce qui s'y joue sans revenir sur les différentes approches del'orientation dans une perspective de genre. Comme évoqué précédemment, le lien étroit entre système dual et marché du travail nécessite également le recours à la littérature de sociologie du travail.

Ces premiers éléments de cadrage ont ainsi permis non seulement de rappeler la spécificité du système suisse de formation professionnelle dual, mais encore de montrer l'abondante littérature nécessaire à son analyse, et enfin de souligner l'importance de procéder à une analyse sous l'angle du genre, tant les enjeux y sont importants et sont restés inexplorés jusqu'ici. 


\section{Chapitre 4 \\ Problématique et méthodologie}

\subsection{Une perspective décentrée}

\subsubsection{Analyser les arrêts de formation pour questionner la transmission des normes de genre}

Cette étude examine, à partir de situations d'arrêts prématurés en formation professionnelle, les processus en jeu durant cette première expérience dans le monde du travail, en particulier la transmission des normes de genre et ses effets. L'analyse portera sur la façon dont ces apprenti.e.s se confrontent à la ségrégation professionnelle, à la division sexuelle du travail et à l'apprentissage des normes de genre. L'objectif est donc de parler de la formation professionnelle comme fabrique du genre à partir des situations d'arrêt, à partir de cas limites. En effet, les situations d'arrêt prématuré, les situations de rupture peuvent être considérées comme des révélateurs de processus en œuvre de manière générale durant la formation professionnelle. La démarche adoptée prend une posture de décentrement ${ }^{53}$, l'enjeu étant de parler de la norme à partir des marges. Ainsi, dans le prolongement de hooks (2000), l'idée est de dépasser le

53 Dans ce sens, l'approche adoptée est comparable à celle de bell hooks (2000), qui revisite la théorie féministe à partir des marges (soit à partir de l'expérience de femmes non blanches et n'appartenant pas à la «middle-class») et qui, se faisant, oblige les théoriciennes des études genre à questionner puis intégrer une perspective intersectionnelle. La démarche renvoie également aux travaux de Mauger et Poliak (1983) qui s'intéressent aux «bandes» de loubards, à leurs relations, leurs interactions, leurs codes et présentent à travers ces éléments des traits largement partagés par les jeunesses populaires, notamment en matière de virilité. Cette démarche ne s'arrête pas à ce premier effet de loupe (les loubards pour parler des jeunesses populaires), mais permet de discuter des normes généralement admises en matière de virilité et le refus de certains traits renvoyant aux milieux populaires. Enfin, la démarche n'est pas sans rappeler les travaux de Pheterson (2001) qui, partant de la situation des prostituées, donne à voir le stigmate qui les touche. L'enjeu est dans un premier temps qu'elles ne soient pas identifiées comme des prostituées dès lors qu'elles ne sont pas en activité (la journée, dans d'autres lieux). Ce stigmate a par ailleurs été utilisé contre les ouvrières, à qui on reprochait le travail salarié hors du ou d'un foyer (domesticité) et 
travail de documentation des «marges», soit l'arrêt d'apprentissage et le processus qui y conduit, pour analyser ce que ces «marges» révèlent du système dans son entier, en l'occurrence la formation professionnelle. Si la démarche de hooks dépasse le seul procédé méthodologique pour questionner théoriquement les travaux et les luttes féministes, en soulignant leurs limites (ne pas intégrer les réalités des femmes en marge de la société), l'enjeu est ici plus modeste. Le processus de décentration opéré permet en effet de voir ce qui constitue la norme, ce dont est fait le quotidien en apprentissage. Il permet donc de porter un regard critique à l'ensemble du système, en partant des situations délicates. Par ailleurs, ce regard décentré sur un objet d'étude a une autre vertu. $\mathrm{Si}$ l'on considère l'arrêt d'apprentissage comme une situation de crise, on peut supposer qu'il exacerbe certains phénomènes, qu'il agit comme un révélateur. Ainsi, les choses se donnent à voir non seulement à la chercheure, mais aussi aux personnes concernées. Cela est d'autant plus important qu'on a affaire ici à une socialisation de genre, qui s'inscrit dans le prolongement de socialisations antérieures (familiales, scolaires, etc.). Cela pourrait rendre la confrontation à la division sexuelle du travail ou encore aux normes de genre relativement invisible. La situation d'arrêt d'apprentissage rend obligatoire pour la chercheure comme pour les jeunes concerné.e.s d'examiner le processus qui y a conduit, et se faisant de reconsidérer les normes transmises, qui dans le cas présent imbriquent socialisation professionnelle et de genre. En partant des arrêts prématurés et de ce que ces situations de crise révèlent des processus qui traversent la formation professionnelle, l'enjeu est donc de cerner de façon plus globale les normes de genre transmises dans ce contexte particulier, ainsi que la manière dont cette transmission se fait, parallèlement à l'apprentissage d'une profession ${ }^{54}$. L'analyse s'intéressera à la manière dont se font ces apprentissages, partant de l'hypothèse qu' ils se font non sans contrainte, voire avec une certaine violence. La brutalité des injonctions à la norme, des remises au pas se donne particulièrement à voir dans les situations d'insertion non-traditionnelle.

enfin, ce stigmate est un outil de contrôle social et de division de l'ensemble des femmes.

54 Les termes métier et profession sont employés comme des synonymes, contrairement à la tradition anglo-saxonne qui distingue entre occupation (métier) et profession (profession). Profession ne renvoie donc pas ici à une activité de travail exigeant un haut degré de formation et disposant d'un monopole d'exercice et de droits juridiques reconnus par l'Etat. 


\subsubsection{Observer les situations pionnières pour faire apparaître les normes dominantes}

Dans la même démarche de décentrement, la recherche se fonde sur l'analyse des situations de personnes ne correspondant pas à la norme de genre dominante, soit parce qu'elles ont investi leur catégorie de sexe différemment (voire qu'elles auraient refusé l'assignation aux normes sociales), soit parce qu'elles sont insérées dans des professions où elles sont minoritaires en regard de leur sexe.

Les personnes en situation pionnière permettent de rendre particulièrement visible l'action des normes de genre. En effet, elles sont en décalage (du point de vue de leur sexe biologique) avec l'identité et les codes sexués d'une profession donnée. Elles transgressent ainsi les frontières de la catégorie de sexe à laquelle elles sont assignées et subissent de ce fait des remarques de collègues. En outre, elles voient attribuer des tâches spécifiques qui sont autant de signes d'une mise au pas, d'un rappel de l'ordre social sexué (Roux, 2006). Le caractère contraignant, parfois violent de ces mises au pas sera mis en évidence, ce qui permettra de discuter du "coût de la transgression" (Couppié et Epiphane, 2007; Marry, 2007). En effet, le choix d'une profession atypique peut être considéré comme une manière de s'éloigner des attentes sociales, des normes. Les personnes ayant opté pour de telles orientations jouent ainsi avec les frontières du genre, même si aucune volonté explicite de transgression ne les anime. On parle alors de coût de la transgression pour parler du prix à payer (en termes d'obstacles rencontrés, mais aussi d'identité, de possibilité d'insertion et de carrière) par ces pionnières et pionniers. Les difficultés rencontrées au moment d'un choix atypique dans l'entourage (soupçon d'homosexualité pour les garçons) ou sur le lieu de travail (résistance des collègues, bizutages, mises à l'épreuve pour les filles) sont autant de rappels signifiant à ces personnes qu'elles sont sorties de leur catégorie de sexe et ont, de ce fait, transgressé les normes de genre ${ }^{55}$.

55 Le coût de la transgression peut conduire au rejet de la profession atypique ou de l'environnement de travail. Cette hypothèse est toutefois débattue. En effet, si les nombreux obstacles rencontrés lors de parcours pionniers (notamment lors de l'insertion professionnelle) laissent penser que le coût peut être assez lourd, une analyse de la situation à moyen terme (stabilisation dans l'emploi) donne une image plus contrastée: d'une part, plusieurs études mettent en évidence des personnes pionnières « heureuses » dans leur profession, car elles ont obtenu des conditions d'emploi favorables (Couppié et Epiphane, 2008; Marry, 2004a). D'autre part, lorsque l'on fait une carrière 
Il s'agit également d'examiner les situations d'apprentissage traditionnelles, où le "sexe du métier » et celui de l'apprenti.e correspondent. Cette analyse doit permettre d'identifier des indices moins immédiatement perceptibles, mais qui signalent néanmoins que le genre est bien transmis parallèlement à la profession. Ainsi, des jeunes hommes devront par exemple apprendre les contours de la «virilité» pour entrer dans le collectif de travail, ainsi que la virilité défensive pour faire face aux difficultés de professions majoritairement occupées par des hommes. Par ailleurs, des jeunes femmes seront confrontées à des normes de "féminité», auxquelles elles ne correspondent pas ou qu'elles refusent.

En insertion atypique, la personne se voit souvent rejetée du milieu professionnel du fait de son sexe. Elle apprend dans cette situation comment est conçue sa catégorie de sexe par les membres de l'autre groupe. Par un effet de miroir, la personne en insertion traditionnelle apprend des autres membres de sa catégorie de sexe ce que signifie être une femme, respectivement un homme. Ainsi, l'analyse de ces deux types de situations permettra de cerner non seulement le contenu des normes de genre, mais aussi la manière dont leur transmission se fait.

\subsection{Une analyse secondaire des données}

Cette recherche s'intéresse en premier lieu à la manière dont le genre traverse toutel'expérience en formation professionnelle, et doncinfluence le processus ${ }^{56}$ qui conduit à un arrêt prématuré. L'enjeu est de questionner le caractère profondément sexué de la formation professionnelle, en ce qu'elle vise à produire de futur.e.s professionnel.le.s qui intégreront un marché du travail fortement ségrégué.

\subsubsection{Hypothèses et questions de recherche}

L'hypothèse principale est que la division sexuelle du travail, les rapports sociaux de sexe et la (non)conformité aux rôles sociaux de sexe parti-

atypique dans un poste ou un métier prestigieux, il semble possible de tenir une position «transgressive » sans en payer le prix (Le Feuvre, 2008; Marry, 2007).

56 Par processus, il est entendu une suite d'événements étalés dans le temps et conduisant à un moment précis: en l'occurrence, la résiliation formelle du contrat d'apprentissage. En outre, la notion de processus a également été retenue pour signifier que divers niveaux agissent sur ce phénomène: individuel, contextuel, etc. Enfin, l'analyse d'une interruption de formation en termes de processus est conforme à la littérature récente sur le décrochage scolaire. 
cipent au processus conduisant aux arrêts de formation, ce tant dans les situations d'insertion pionnière ${ }^{57}$ que dans les situations d'insertion traditionnelle. L'idée n'est pas d'affirmer que les normes de genre ou leur transmission sont la cause des arrêts prématurés ${ }^{58}$, mais de souligner que le genre traverse l'entier de l'expérience de la formation professionnelle, y compris le processus qui conduit à l'interrompre.

L'enjeu est d'appréhender les différences (ou l'absence de différence) entre les jeunes femmes et les jeunes hommes dans la façon d'expliquer et de vivre un arrêt de formation professionnelle, compte tenu du fait que leurs expériences s'inscrivent dans des contextes professionnels sexués.

La deuxième question de recherche interprète la formation professionnelle comme un lieu de production et de transmission du genre. Elle a pour objectif de cerner les normes professionnelles marquées par le genre et de comprendre comment celles-ci sont apprises au cours de l'expérience de formation. Si l'apprentissage permet de devenir un.e professionnel-le, une personne du métier (quel qu'il soit), comment conduit-il aussi à devenir un homme ou une femme conforme aux rôles sociaux de sexe, tenant sa place dans les hiérarchies de genre? Il s'agit donc d'analyser la manière dont les normes de genre sont transmises, comment l'assignation à une place sexuée se fait.

\subsubsection{Une approche qualitative qui s'impose}

Ce travail procède à une nouvelle analyse des entretiens menés dans le cadre d'une recherche sur les arrêts prématurés en formation professionnelle (Lamamra et Masdonati, 2009). Cette analyse secondaire intègre une perspective de genre inédite. Cette perspective s'est imposée au fil de l'examen des données sur les arrêts prématurés, car la formation professionnelle est traversée tant par les inégalités produites par le système éducatif, que par la ségrégation du marché du travail fondée sur le sexe. Les analyses secondaires intègrent donc des données qui n'étaient pas encore exploitées et propose une réflexion originale sur les arrêts, et plus largement sur la formation professionnelle comme lieu de fabrication du genre. On retrouvera cependant, bien que complètement revisitées par la lecture sous l'angle du genre, les thématiques de l'étude sur les arrêts prématurés: raisons de l’arrêt, souffrance, stratégies.

57 Les termes insertion pionnière, insertion atypique, métier ou profession atypique et par extension métier pionnier ou profession pionnière sont ici utilisés de manière indifférenciée.

58 L'analyse n'a pas porté sur l'hypothèse d'arrêts dus au système de genre, mais certaines situations pourraient être interprétées ainsi. 
L'approche qualitative, privilégiée dans la recherche sur les arrêts prématurés en formation professionnelle, a semblé particulièrement pertinente pour l'analyse secondaire. En effet, en étant à même d'appréhender les processus fins menant à un arrêt prématuré en formation professionnelle, l'approche qualitative a permis de comprendre les processus de socialisation professionnelle et de genre, en particulier la manière dont les normes de genre se transmettent au cours du passage en formation professionnelle.

\section{Les entretiens semi-directifs}

La recherche se base sur des entretiens semi-directifs menés auprès de 46 jeunes ayant interrompu leur formation professionnelle dans le canton de Vaud durant leur première année d'apprentissage ${ }^{59}$. Les jeunes ont été rencontré.e-s dans un délai relativement bref après leur arrêt prématuré, soit dans les trois mois qui ont suivi la résiliation du contrat ${ }^{60}$. Les entretiens - d'une heure à une heure et demie - ont été organisés autour de différents moments: dans un premier temps, et hors enregistrement, l'information portait sur les caractéristiques sociobiographiques des apprenti.e.s (formation des parents, fratrie, parcours antérieur, etc.), puis, le canevas d'entretien permettait d'aborder les raisons de l'arrêt, les questions relationnelles (sur le lieu de travail, à l'école professionnelle), biographiques (orientation professionnelle, projets) et systémiques (autres sphères de vie, loisirs). L'entretien se terminait par des informations factuelles sur la situation des jeunes au moment de la rencontre (en recherche d'une place d'apprentissage ou d'emploi, en formation, etc.). Les entretiens ont été intégralement retranscrits et soumis à une analyse de contenu thématique (Bardin, 1986).

La collecte des données n'a pas directement intégré une perspective de genre. La seule démarche qui a été effectuée en ce sens concerne la sélection de la population, où une stricte parité hommes - femmes a été observée. Le canevas d'entretien n'incluait aucune question explicite en termes de genre, que ce soit dans le choix de la formation ou de la place

59 Près de $60 \%$ des résiliations de contrat intervenant en 1 ère année (Stalder et Schmid, 2006a), la population de la présente étude n'a intégré que des jeunes ayant connu un arrêt au cours ou au terme de leur première année.

60 S'agissant d'une analyse secondaire de données collectées dans un projet de recherche collectif, je précise ici que 24 des 46 entretiens ont été menés par mes soins. Les autres ont été effectués par le co-auteur de la recherche, Jonas Masdonati, et par deux collègues, impliquées dans le projet, Elodie Chevalier et Jacqueline De Puy. 
d'apprentissage ou encore dans les problèmes rencontrés durant cette première expérience sur le marché du travail. Une question portait de manière générale sur le sentiment d'inégalité ou de discrimination, mais elle dépassait les seules inégalités de sexe, puisqu'elle se proposait aussi de débusquer les inégalités de traitement en lien avec la nationalité, la filière suivie au secondaire ou de manière plus générale avec le statut d'apprenti.e. Cette question offrait cependant aux jeunes la possibilité de faire état de discriminations liées au sexe.

Si le canevas n'incluait aucune question spécifique, lors des entretiens, les relances ont permis de rebondir sur l'évocation de certaines discriminations: harcèlement sexuel, sexisme, inégalités de traitement. Les situations pionnières ont également bénéficié d'une attention particulière. Ainsi, on retrouve au centre des analyses présentées dans cet ouvrage, une multiplicité de situations discriminatoires, énoncées sans "stimulus » particulier. Cette démarche révèle ainsi la force du système de genre et sa transversalité dans le champ du travail et de la formation.

\section{Une relecture des données par le prisme du genre}

Pour l'analyse secondaire, le matériel transcrit a été intégralement relu et recodé. Des extraits déjà identifiés ont été soumis à de nouvelles analyses en termes de genre. L'analyse secondaire, tout comme l'analyse initiale, a été le fruit d'une double procédure déductive et inductive. Elle a en outre porté sur des réflexions plus approfondies, en particulier sur les biais d'entretien et la manière dont les jeunes del'un ou l'autre sexe rapportent leur expérience en formation professionnelle. Dans un premier temps et afin de se réapproprier le matériel pour une analyse sousl'angle du genre, le corpus a été séparé par catégorie de sexe. Les différentes thématiques précédemment identifiées (raisons de l'arrêt, type de souffrance, type de stratégie) ont ainsi été soumises à une comparaison de fréquences entre filles et garçons. La même démarche a été retenue pour distinguer l'expérience des personnes pionnières de celles des jeunes inséré.e.s de manière traditionnelle ${ }^{61}$. Cependant, cette approche chiffrée a très vite montré ses limites (en ce qu'elle ne révélait guère de différences et comportait le risque d'une réification des catégories de sexe) et l'essentiel de la réflexion porte sur l'analyse de contenu thématique.

61 Le comptage des personnes insérées dans des métiers atypiques a posé des problèmes spécifiques. En effet, aux personnes insérées dans des métiers pionniers s'ajoutent encore les personnes en situation pionnière (dans un métier traditionnel pour leur sexe, mais dans des environnements où elles étaient minoritaires). Toutes ces situations ont été analysées ensemble. 


\section{Une posture compréhensive nécessaire à un objet d'étude particulier}

La posture de la recherche est une posture compréhensive (Rogel, 2004). L'entier de la démarche a composé avec la subjectivité et le primat de la parole des actrices et des acteurs. Ce positionnement s'est illustré tant dans les questions de recherche, que dans l'analyse de genre transversale ou dans le recours à l'approche de la psychodynamique du travail, utilisée pour traiter de la souffrance et des stratégies développées pour y faire face. Bien que l'analyse se fonde sur le discours subjectif des jeunes interviewé.e.s, elle ne néglige pas pour autant l'examen des interactions entre chercheur.e.s et personnes interviewées, permettant ainsi de développer une réflexion sur la manière dont les jeunes femmes et les jeunes hommes restituent leur expérience, soit comment l'entretien est l'occasion d'une «parade de genre» (Goffman, 2002). Enfin, une perspective plus macrosociale a également été adoptée, ce afin de tenir compte des aspects contextuels et structurels qui déterminent les situations vécues par les apprenti.e.s. En effet, certaines expériences nécessitent d'être appréhendées dans le contexte plus global du marché de la formation professionnelle étroitement lié au marché du travail et traversé par les inégalités et la ségrégation. Pour autant, l'analyse reste centrée sur les apprenti.e.s et ne propose ni d'analyse institutionnelle des mécanismes de production et reproduction de normes de genre en formation professionnelle, ni d'analyse du cadre législatif visant à changer la donne en matière de ségrégation dans la formation professionnelle (par exemple projet 16+, TEKNA, etc.).

\subsubsection{Population}

Les 46 entretiens semi-directifs (voir Annexe 3) ont été menés sur une base volontaire. Afin d'avoir une population variée, des quotas ont permis de tenir compte de trois variables: le sexe, la filière suivie au secondaire I et le secteur professionnel. En l'occurrence, la parfaite parité femmes hommes ainsi obtenue, soit 23 personnes de chaque sexe, s'est révélée très utile pour mener les analyses secondaires.

La très grande majorité des apprenti.e.s interviewé.e.s provient d'une filière du secondaire à exigences moyennes (VSG, voie secondaire générale) et d'une filière à faibles exigences (VSO, voie secondaire à options $)^{62} .7$ autres apprenti.e.s n'ont pas passé par ces filières vaudoises,

62 Au moment de l'étude, le système du secondaire vaudois était organisé en trois filières, dans lesquelles les élèves étaient orienté.e.s selon leurs perfor- 
ayant suivi leur scolarité obligatoire dans un autre canton, un autre pays, ou provenant de filières à effectifs réduits ou de classes d'accueil pour migrant.e.s (pour le détail, voir Annexe 3).

Un quota a également été fixé concernant les secteurs d'activité. Bien que les places d'apprentissage soient plus nombreuses dans certains secteurs, il importait d'avoir une palette qui rende compte des divers secteurs professionnels : l'industrie, le bâtiment, la vente, le commerce, les métiers verts et les soins. Des domaines d'activité tant féminisés, que masculinisés et mixtes (tels que le commerce, même si à l'intérieur de ce secteur, une ségrégation horizontale des types d'emplois est reconstruite) ont ainsi pu être touchés. Cela a permis de questionner la transversalité du système de genre. Compte tenu de la ségrégation en vigueur (OFS, 2008b), certains bastions, soit des métiers fortement sexués, se dégagent (voir Annexe 4).

Il n'y a pas eu de tentative de croiser les différents quotas entre eux (par exemple sexe et secteurs professionnels), ce dans le but d'avoir un nombre comparable de filles et de garçons par secteur. Ce travail aurait été non seulement difficile, mais encore peu pertinent.

Quatre autres variables ont été prises en considération dans la sélection de la population: la nationalité, l'âge, les catégories socioprofessionnelles des parents et le parcours antérieur des jeunes.

La majorité des jeunes, soit 35 sur les 46 rencontré.e.s en entretien, sont de nationalité suisse et bi-nationale, celles et ceux issu.e.s de la migration ayant le plus souvent refusé de participer à l'étude (pour le détail, voir Annexe 3). Comme certains travaux ont relevé la plus forte fréquence de rupture de contrats d'apprentissage chez les jeunes d'origine étrangère et leur surreprésentation parmi les personnes sans formation (Dubs, 2006; Eckmann-Saillant, Bolzman et De Rham, 1994; Meyer,

mances scolaires : la voie secondaire baccalauréat (VSB) est la filière la plus exigeante et qui était destinée, comme son nom l'indique, à une poursuite au secondaire supérieur académique sanctionné par l'obtention d'une maturité fédérale (baccalauréat); la voie secondaire générale (VSG) une filière à exigences moyennes et la voie secondaire à options (VSO) une filière à exigences faibles. Cette catégorisation reprend la classification proposée par le Service d' d'orientation cantonal du canton de Berne et utilisée par l'équipe du SRED (Rastoldo et al., 2009; Rastoldo et al., 2007). Ce système à trois voies distinctes a été aboli en septembre 2014, lors de l'adoption de la LEO (Loi sur l'enseignement obligatoire) en votation populaire. Traditionnellement, les deux filières VSO et VSG étaient les principales pourvoyeuses dela formation professionnelle (voir SCRIS: http://www.scris.vd.ch/Default. aspx ?DocID=6939\&DomId=2512). 
2005), on peut soupçonner un biais dans la population. La sélection se faisant sur une base volontaire, l'accès plus difficile à ces jeunes suggère qu'elles et ils se sentent moins légitimes que les Suisses-ses à prendre la parole et à se rendre visibles (Nicole-Drancourt et Roulleau-Berger, 2002).

Les jeunes interviewé.e.s ont entre 15 et 23 ans, la moyenne se situant à 17,5 ans. Cet âge moyen relativement élevé est conforme aux dernières tendances en matière d'allongement de la transition et d'âge d'entrée en formation professionnelle (Meyer, 2005; OFS, 2008a). En reprenant la catégorisation de Arnett (2000), qui différencie les adolescent.e.s des adultes émergent.e. $\mathrm{s}^{63}$ sur la base d'un faisceau d'éléments développement, législation, sociabilité, mode de vie, etc. -, la population se compose de 30 adolescent.e.s (de 15 à 17 ans) et 16 adultes émergent.e.s (de 18 à 23 ans) (pour le détail, voir Annexe 3).

Les parents sont majoritairement des salarié.e.s qualifié.e.s $(\mathrm{N}=54)$. Cependant, un nombre non négligeable sont des personnes non qualifié.e.s $(\mathrm{N}=15)$ et une minorité sont des mères au foyer $(\mathrm{N}=5)$. Plusieurs sont de petit.e.s indépendant.e.s $(\mathrm{N}=9)$ et un seul occupe une position dirigeante. La majorité des familles concernées, qu'elles soient ouvrières, employées ou de petit.e.s indépendant.e.s ont donc une expérience de la formation professionnelle (pour le détail, voir Annexes 3 et 5).

Enfin, confirmant d'autres travaux sur les arrêts prématurés (Rastoldo, Kaiser et Evrard, 2008), la grande majorité des jeunes connaît un parcours antérieur non-linéaire soit au cours de la scolarité obligatoire (redoublement, changement d'orientation, $\mathrm{N}=22$ ), soit après (structure de transition au sortir du secondaire I, précédent arrêt de formation professionnelle, changement d'orientation, etc., $\mathrm{N}=24$ ). Ainsi, seules 12 personnes ont connu jusque-là un parcours parfaitement linéaire (Lamamra et Masdonati, 2009).

\section{Accès à la population}

Il est difficile d'avoir accès à des jeunes ayant interrompu prématurément leur formation. En effet, ces ex-apprenti.e.s n'étaient plus en entreprise et pour une part avaient également quitté l'école professionnelle. Pour des questions de protection des données, il était en outre impossible d'obtenir la liste des personnes concernées par une résiliation de contrat d'apprentissage et établie régulièrement par les autorités cantonales. Aussi, l'accès à cette population a-t-il été rendu possible grâce à l'association TEM - Transition Ecole Métier, qui a pour mandat de contacter

63 Plus précisément, cet auteur distingue les adolescent.e.s (de 10 à 17 ans) des adultes émergent·e.s (de 18 à 25 ans). 
l'ensemble des jeunes du canton de Vaud touchés par une résiliation de contrat. C'est par leur intermédiaire que la recherche a été présentée aux jeunes, et ce n'est qu'après l'accord des apprenti.e.s qu'un premier contact était pris. Il s'agit donc d'une démarche sur base volontaire, ce qui n'est pas sans créer quelques biais.

Deux cas de figure doivent être signalés ici. Tout d'abord la surreprésentation de jeunes suisses.ses laisse penser que pour les immigré.e.s, notamment celles et ceux récemment arrivé.e.s en Suisse, il y a une barrière, un frein. En effet, elles et ils n'ont pas nécessairement le sentiment d'avoir une parole autorisée (Nicole-Drancourt et Roulleau-Berger, 2002). Ensuite, il est probable que certains cas de figure aient complètement échappé à l'a nalyse. En effet, des jeunes ayant des situations socialement stigmatisantes ou jugées moins défendables - difficultés familiales, toxicodépendances ou problèmes avec la justice - n'ont peut-être pas osé s'exposer et parler de leur situation. Pour autant, la population interviewée présente un intérêt certain pour l'analyse, de par l'hétérogénéité des profils rencontrés. En outre, la démarche qualitative permettant une analyse fine des situations rencontrées, la richesse des témoignages constitue un apport indéniable de cette recherche.

\subsection{Apports et limites d'une analyse secondaire}

En proposant une perspective de genre sur des données qualitatives, l'analyse secondaire a permis de percevoir des éléments que les précédentes études sur les ruptures d'apprentissage ne sont pas parvenues à faire apparaitre. Outre que certaines d'entre elles ne ventilent pas leurs données par sexe (Rastoldo, Amos et Davaud, 2009), celles qui le font rendent compte de différences minimes entre filles et garçons, statistiquement non significatives, à l'instar de l'étude LEVA (Schmid, 2010). Dès lors, ces travaux suggèrent une certaine similarité dans les parcours menant à l'arrêt. Alors que, dès lors que des analyses de contenus plus fines sont effectuées, des différences apparaissent. Par exemple, les « relations au travail» ne recouvrent pas les mêmes réalités pour les filles et les garçons: leurs attentes en la matière diffèrent et le contexte qui produit ces différences change lui aussi selon le secteur professionnel (métiers de service, métiers de l'industrie) dans lequel les jeunes sont inséré.e.s. Comme le genre se joue en permanence dans les rapports sociaux (West et Zimmerman, 1987; Stalder et Schmid, 2006a), les relations entre un.e maître.sse d'apprentissage et son apprenti.e sont marquées par leurs positions sociales dans la hiérarchie de l'entreprise, ainsi que 
par celles occupées dans l'ordre hiérarchique entre les sexes. Dans une relation dysfonctionnelle par exemple, on attaquera la virilité d'un jeune homme, pendant qu'une jeune femme subira du harcèlement sexuel. Les expériences vécues par les apprenti-e.s ne sont donc pas identiques: elles sont structurées par le genre, la ségrégation professionnelle et la division sexuelle du travail.

En travaillant sur ces différences, l'objectif n'est pas de réifier les catégories de sexe et de les penser comme nécessairement antagonistes, ni d'entamer une démarche dictée par le postulat largement partagé de l'antériorité du sexe sur le genre et dénoncé par Delphy (2001). L'accent est mis ici sur le «comment ça se passe» et ce faisant, sur la façon dont les pratiques en usage dans le champ de la formation professionnelle à l'égard des apprenti.e.s produisent du féminin et du masculin.

Le fait que la perspective de genre n'ait pas été pensée lors de l'élaboration du canevas d'entretien et qu'elle n'ait été que partiellement à l'esprit des chercheur.e.s au cours des entretiens créent certaines limites. Il manque parfois en arrière-plan les différentes réalités professionnelles (secteurs d'activité, positions dans les emplois). Ainsi, le lien entrel'expérience rapportée, par exemple la souffrance, et le contexte spécifique qui la fait émerger (Molinier, 2000) n'a pas toujours été abordé directement, il a été reconstitué à posteriori.

Par ailleurs, il est délicat de décoder ce qui se joue dans l'interaction chercheur.e et interviewé.e, notamment en matière de genre. Les jeunes ayant déjà été socialisé.e.s de façon différenciée avant leur entrée en formation professionnelle, des "parades de genre» étaient probablement à l'œuvre lors des entretiens. Aussi, certaines réponses reflètent-elles avant tout un besoin de conformité aux attendus sociaux en matière de genre (femme "féminine» qui parle de sa souffrance, homme «viril» qui la tait).

En ce qui concerne l'analyse secondaire, la tentation de procéder à une comparaison femmes-hommes était grande, du fait notamment du partage paritaire de la population sur la base du sexe. Omniprésente dans la littérature, y compris en études genre, cette démarche a tendance à réifier la catégorie femmes, dès lors opposée ou comparée à la catégorie hommes. Ce faisant, la catégorie de sexe devient une catégorie pertinente d'analyse, au travers de laquelle on cherche à faire apparaître des différences. Du genre est ainsi créé, là où l'analyse aurait souhaité faire apparaître la manière dont il se construit (Alvarez, 2005). Dans un premier temps, j'ai procédé par comptage, bien qu'étant engagée dans une démarche qualitative, afin de voir si des différences se dégageaient 
entre les deux corpus. C'était parfois le cas, mais le plus souvent les similitudes entre les sexes étaient plus nombreuses. Ce n'est qu'en passant par de nouvelles analyses de contenu plus fines que les différences observées ont pu être fondées. C'est donc au travers des récits des jeunes que l'analyse a permis de révéler la façon dont se construit le genre au cours de l'expérience qui conduit à l'arrêt prématuré en formation professionnelle. Cela a permis de voir au-delà des différences, certaines similitudes dans les parcours (Rosende, 2008). A partir de ces similitudes, il était dès lors particulièrement intéressant de chercher à comprendre comment l'expérience de l'arrêt marque les jeunes et participe à créer du genre (dans le sens de "doing gender») (West et Zimmerman, 2009).

Enfin, le dernier aspect à souligner concerne également l'analyse. L'objectif premier étant de mettre l'accent sur la façon dont le genre se construit au cours de l'expérience en formation professionnelle, d'autres rapports sociaux n'ont guère été abordés et surtout aucune articulation systématique des diverses formes de domination n'a été proposée (Jaunait et Chauvin, 2012; Martin et Roux, 2015; Sudbury, 1998). Les limites des informations à disposition (catégories socioprofessionnelles des parents reconstruites à partir de données très lacunaires) ou les biais d'accès à la population (très peu de jeunes d'origine immigrée) ont rendu ce travail particulièrement difficile. Cependant, lorsque les informations disponibles ont rendu cette démarche possible, certaines imbrications ont été soulignées, à l'instar de la hiérarchie entre cols blancs et cols bleus, du rapport de subordination lié au statut d'apprenti.e, du racisme comme stratégie de défense face aux violences sexistes ou encore l'articulation entre système de genre et hétéronormativité. 


\section{Chapitre 5 \\ Arrêter sa formation professionnelle: motifs et processus}

Si les travaux sur les arrêts prématurés en formation professionnelle sont peu nombreux en Suisse et abordent des aspects divers, ils font généralement consensus sur les causes des résiliations anticipées de contrat d'apprentissage. Ces raisons sont multiples et ont fréquemment fait l'objet d'analyses comparées, les motifs évoqués par les apprenti.e.s étant confrontées à ceux annoncés par les entreprises formatrices (Schmid, 2010). Ces raisons se divisent globalement en deux catégories: les arrêts motivés par le choix du métier ou de l'entreprise et ceux liés aux conditions de travail et/ou de formation dans l'entreprise (voir Chapitre 2). A cela s'ajoutent les raisons qualifiées par les chercheur.e.s comme relevant du "personnel» (Schmid, 2010) et obligeant des apprenti.e.s à mettre un terme à leur formation. Dans la confrontation des points de vue entre apprenti.e.s et entreprises formatrices, les un.e.s mettentl'accent sur les conditions de travail et de formation, ainsi que sur des situations conflictuelles au sein de l'entreprise, tandis que les autres soulignent le manque de motivation et les mauvaises performances scolaires des jeunes (Schmid et Stalder, 2007; Stalder et Schmid, 2006a).

Il s'agit encore de souligner ici que l'arrêt n'est que rarement provoqué par une seule cause, c'est un cumul de raisons qui conduit, en effet, une personne à mettre un terme à sa formation.

Sur la base des entretiens qualitatifs menés dans la recherche, ce chapitre analyse d'une part le contenu des différentes raisons ${ }^{64}$, en cherchant à mettre au jour les réalités que les catégories assezglobales évoquées ci-dessus tendent à masquer, et, d'autre part, d'identifier les enjeux de

64 Deux remarques s'imposent. Tout d'abord, seules les raisons évoquées pour expliquer l'arrêt de formation sont exposées, et non les aspects positifs de la formation relatés par les jeunes interviewé.e.s, ce qui ne signifie pas que ces derniers et dernières soient absent.e.s des témoignages. Ensuite, étant donné que les analyses se basent exclusivement sur les récits des ex-apprenti-e.s, elles peuvent donner l'impression que les arrêts de formation ont exclusivement été à l'initiative des jeunes. Cela n'est assurément pas le cas, un arrêt de formation étant généralement le résultat d'un processus impliquant les différent.e.s acteurs et actrices de la formation professionnelle (formateurs et formatrices, enseignant·e.s, patron'ne.s, etc.). 
genre qui sous-tendent les raisons de l'arrêt. En effet, la sexuation des filières de formation, ainsi que la ségrégation professionnelle horizontale font que les réalités professionnelles auxquelles sont confrontées filles et garçons diffèrent fortement. Inscrit.e.s dans des secteurs très distincts, les jeunes se retrouvent face à des contraintes professionnelles diverses: pression d'un important volet relationnel dans les métiers de service pour les unes, pénibilité, rudesse et pression à la logique de production des métiers «traditionnellement» investis par les hommes (bâtiment ou industrie) pour les autres. Difficile donc de ne pas lier les arrêts à ces contraintes spécifiques. Pourtant, dans les travaux actuels sur les résiliations de contrat d'apprentissage, cette dimension n'apparaît pas, comme si elle n'influait en rien sur le processus conduisant à un arrêt. Ce chapitre a donc pour objectif de faire émerger - derrière des raisons apparemment similaires - des réalités marquées par le genre.

Les raisons de l'arrêt de formation ont été regroupées en cinq catégories, qui recoupent en bonne partie celles mises en évidence dans la littérature (voir Chapitre 2): des difficultés liées à l'apprentissage $d u$ métier, des problèmes rencontrés au niveau des interactions et des relations au travail, des problèmes rattachés au monde du travail (conditions de travail, santé, etc.), des difficultés dans la transition entre école obligatoire et formation professionnelle et des raisons motivées par des contingences externes. La présentation détaillée de chacune de ces catégories permettra d'illustrer leur forte hétérogénéité. En effet, sont regroupées dans une même catégorie des situations très diverses.

\subsection{L'impossibilité d'apprendre le métier}

Le trois quart des personnes interviewées expliquent leur arrêt de formation par des difficultés liées à l'apprentissage du métier $(\mathrm{N}=34)$. Dans cette catégorie sont regroupées les difficultés liées à l'impossibilité ou à l'incapacité d'apprendre le métier choisi, qui peuvent donc être résumée ainsi: d'une part, les situations liées à des difficultés du point de vue de la performance scolaire $(\mathrm{N}=10)$, d'autre part les situations où les conditions de formation sont inadéquates $(\mathrm{N}=24)$. Cette inadéquation de la formation peut prendre plusieurs formes: un encadrement lacunaire, voire inexistant, une charge de travail insuffisante, un décalage entre ce qui est fait en entreprise et ce qui est appris à l'école professionnelle ou encore des situations d'exploitation.

L'impossibilité d'apprendre le métier n'apparaît jamais comme raison unique d'un arrêt d'apprentissage et s'articule à d'autres raisons, 
en particulier les relations au travail ou ce qui relève des conditions de travail.

\subsubsection{Les performances scolaires}

La question des performances fait généralement consensus dans les diverses études, ainsi qu'entre apprenti.e.s et formateurs ou formatrices en entreprise, les deux parties s'accordant facilement lorsqu'un arrêt est dû à des résultats insuffisants ou à un échec aux examens. Pourtant, bien que différentes études l'identifient comme raison essentielle (voir Kaiser et al., 2007; Stalder et Schmid, 2006a), elle apparait peu ici et semble surtout être une conséquence d'autres problèmes. Ce motif étant davantage évoqué par les apprenti.e.s d'origine étrangère (C. Moser et al., 2008), la composition de la population (voir Chapitre 4) pourrait expliquer cette faible fréquence. En outre, il est davantage relevé par les garçons. Sur les 10 jeunes expliquant leur arrêt par des performances insuffisantes, huit sont des garçons.

Certaines situations paraissent assez claires, l'arrêt intervenant suite à l'échec aux examens ou à une moyenne insuffisante durant l'année. Cela est le cas pour Hakem, un réparateur automobile de 19 ans:

Je voulais continuer, mais c'est à la fin de l'année, là, l'examen de première année. En fait, j'ai pas eu le résultat suffisant pour passer en deuxième.

[...] Etj'ai discuté avec le patron, si il me laissait refairel'année, pis il a dit: "Je retire le contrat. ${ }^{65}$

65 Afin de restituer au plus près les propos des jeunes, ils ont été très peu «toilettés». La forme orale a été maintenue, car elle donne à voir le statut des personnes interviewées (Rogel, 2004). En outre, comme évoqué dans le Chapitre 4, la recherche a été menée avec une posture compréhensive favorisant le primat de la parole des acteurs et des actrices. C'est également dans cette perspective que les propos des jeunes ont été restitués le plus fidèlement possible. Faire entrer leurs propos dans un langage écrit normatif aurait constitué un renforcement de la position asymétrique entre d'un côté des chercheur.e.s, adultes autorisé.e.s et de l'autre des interviewé.e.s, adolescent.e.s, en situation de rupture professionnelle. Enfin, s'agissant de propos relatant une expérience souvent difficile, les hésitations, difficultés d'énonciation peuvent être aussi significatives que les termes utilisés, même si ce type d'analyse (champ lexical, linguistique ou sociolinguistique) n'a pas été développé ici. 
Dans d'autres cas, comme celui de Yvan, un employé de commerce de 17 ans, l'employeur pose des exigences plus élevées que celles posées par l'école:

En fait, j'ai pas respecté une convention d'objectifs, que mon entreprise m'avait fixée, parce que j'avais pour eux des notes insuffisantes, donc entre 4 et 4.5. Pour eux, ils voulaient plus, pis ils m'ont fixé, en fait, des notes, je devais pas descendre en dessous de 4.5...66

Les jeunes expliquent parfois ces difficultés scolaires par un écart important entre les exigences du secondaire I (l'école obligatoire) et les contenus ou le niveau demandé aux cours à l'école professionnelle. Ces difficultés sont évoquées sans gêne, c'est le cas par exemple de Bastien, un monteur-électricien de 15 ans:

Parce que ce qu'on fait c'est presque niveau VSG, quoi, supérieur. [...] Aux cours là, souvent j'étais largué, je comprenais plus rien quoi. [...] Ben aussi, faut dire que je suis dans l'âge con entre guillemets [rit], pis on n'a pas tellement envie de travailler les cours quoi. C'est ce qui m’a fait louper.

De telles situations, qui concernent surtout les jeunes issu.e.s de la filière la moins exigeante (VSO) sont donc à situer entre le type de raisons rattachées à l'apprentissage du métier, présenté ici, et celles motivées par des difficultés de transition, qui seront présentées plus loin. Le témoignage de Raphaël, employé de commerce de 16 ans, illustre bien ce genre de difficultés:

Y a juste l'informatique où je me débrouillais assez bien, où je me débrouille bien mais autrement l'allemand, l'anglais, le français, ça, j’ai jamais vu à l'école.

[...] Avant, à l'école, j'étais fort en français, mais c'était en orthographe, la conjugaison, des choses comme ça, tandis que maintenant c'est tout de la littérature. Ça, j’ai jamais fait à l'école!

Soulignons que ces problèmes se situent toujours au niveau des cours théoriques, dans la plupart des cas le travail en entreprise ne pose pas de problèmes en termes de performances. Ainsi, Hakem, Bastien et Yvan, cités ci-dessus, affirment respectivement:

66 En Suisse, la notation se fait sur 6, la moyenne étant à 4. 
Pour moi, c'est juste les cours, parce que [en] pratique je me débrouille bien. (Hakem)

Les cours, c'est le seul truc quoi, parce que sinon tout jouait bien quoi. (Bastien)

Le travail, autrement, ça se passait très bien! On m’a même dit qu'il y avait pas de problèmes au travail, c'était à cause de ces petits problèmes aux cours. (Yvan)

Il est également intéressant de relever que la plupart des jeunes ayant évoqué des problèmes de performances scolaires font tout leur possible pour combler ces lacunes, ce qui montre leur engagement dans la formation. Bastien, le monteur-électricien précité, a pris conscience qu'un tel effort était nécessaire:

Français, j'avais 4, maths, j'avais 3.5, électrotechnique, j'avais, euh, je devais avoir 3, bon, je prends des cours, justement, le samedi matin, j'ai eu des cours, là j'ai une heure par semaine, pis justement je prends des cours exprès pour me relancer dans la profession, et réussir mieux.

[...] Je me suis rendu compte qu' il fallait vraiment bien bosser, et qu'il fallait passer du temps sur les cours et tout, mais j'ai compris ça trop tard quoi.

Ce jeune homme met en évidence différents aspects liés aux difficultés rencontrées dans le volet scolaire de la formation professionnelle. Tout d'abord, il se heurte à un niveau plus exigeant qu'au secondaire, cela est d'autant plus marqué dans son cas qu'il est issu d'une filière à faible niveau d'exigence (VSO). Ensuite, à l'instar d'autres jeunes en situation d'échec scolaire en formation professionnelle, il relève que le volet métier ne pose pas de problème particulier. Enfin, la transition école-formation professionnelle lui paraît difficile. En effet, les exigences particulières du système dual, soit gérer son temps entre école et entreprise, organiser son travail scolaire d'une semaine à l'autre sont autant d'éléments qui peuvent poser problèmeà des adolescent.e.s ayant jusque-là eu l'habitude d'un cadre scolaire très structuré.

\subsubsection{Les conditions de formation}

La question des conditions de formation est centrale. Dans 24 cas où des problèmes d'apprentissage du métier sont signalés, la raison de l'arrêt ne se pose pas en termes de performances scolaires, mais d'impossibilité 
d'apprendre le métier dans de bonnes conditions. Cela renvoie donc à des problèmes liés à la conception et à l'organisation de la formation au sein de l'entreprise ${ }^{67}$. Cette problématique a également été soulevée en France par Moreau (2003), qui met en évidence certaines carences en termes de prise en charge de l'apprenti.e au sein des entreprises de formation. Dans ses recherches, la question du manque est fréquente dans les récits des apprenti.e.s: manque de temps, de présence et de suivi de la part de la formatrice ou du formateur, manque de feedbacks sur le travail fourni, manque de travail pour l'apprenti.e, manque de droit à l'erreur ou encore manque de coordination entre l'école et l'entreprise. La plupart de ces manques se retrouvent ici (mauvais ou absence d'encadrement, charge insuffisante de travail, décalage entre l'entreprise et l'école). A cela s'ajoutent des cas « extrêmes » qui révèlent des situations d'exploitation de l'apprenti.e.

En l'absence d'encadrement, certain.e.s sont livré.e.s à elles- ou eux-mêmes et doivent s'auto-former. Elles et ils doivent également mettre en place des stratégies pour éviter l'ennui. Dans de telles situations, le métier ne peut pas s'apprendre correctement. Ceci est notamment le cas pour Amina, une gestionnaire de vente de 17 ans:

Des fois, il [formateur en entreprise] était même pas au magasin. Il partait, il revenait, il venait juste pour voir. Et pis des fois, le vendredi, l'apprenti [le deuxième dans l'entreprise] il est au cours, moi j'étais toute seule.

[...] Il était jamais au magasin, lui, son boulot c'était venir voir et pis après partir. En fait, [...] c'était tout l'apprenti qui me montrait. C'était même l'apprenti qui m'avait sorti le contrat, tout ça. Lui [le formateur en entreprise], il faisait rien. Lui, il était là : «Oui, d'accord, c'est bon, vous pouvez l'engager! (68 $^{68}$

67 La formation dispensée à l'école professionnelle n'apparaît quasi pas lorsque sont évoquées les conditions de formation. Comme pour la plupart des raisons présentées par les jeunes, le volet école du système dual n'est presque jamais abordé.

68 Pour plus de clarté: le formateur en entreprise dit au second apprenti, qu'il peut engager l'apprentie gestionnaire de vente interviewée. C’est une manière d'une part pour la jeune femme de souligner le désinvestissement de son formateur, mais également pour ce-dernier de signaler la hiérarchie existant entre les deux apprenti.e.s: le garçon étant de fait placé dans une position quasi équivalente à celle du formateur/employeur. 
Stella, une esthéticienne de 17 ans, vit une situation similaire. Sa formatrice en entreprise est absente pour cause de maladie et la jeune femme tente de s'auto-former:

Elle [la formatrice en entreprise] est partie en congé maladie, pendant 5 mois. Et puis, ben, j'avais personne pour me former, parce que l'autre esthéticienne, elle travaillait à $100 \%$ pis c'est pas une formatrice, donc.

[...] Et pis, ben, je regardais quand j'avais personne, j'avais du temps libre, ben j'essayais de regarder ce que faisait l'autre. Et pis, je suis assez visuelle, donc j'apprends assez vite quand je regarde.

Ces situations d'auto-formation renvoient très précisément à ce que Moreau (2000) souligne comme enjeu majeur pour les filles, à savoir dans un premier temps, l'accès au métier et au savoir-faire. Tout se passe, en effet, comme si elles devaient "voler leur métier» (Scott, 1991). Pour accéder aux savoirs professionnels, elles cherchent la formation auprès de collègues qu'elles observent, elles apprennent sur le tas.

Certains garçons se retrouvent également en situation de manque total d'encadrement, comme Hakem, un réparateur automobile de 19 ans, qui doit organiser lui-même son travail:

En fait à la fin, c'est moi-même qui allait vers les mécaniciens, je leur disais: "J'ai envie de faire ça, j'ai envie de faire ça», et même si les chefs d'atelier ils disaient quelque chose, je disais: «Non!», puis je continuais à faire mon travail, et dès que je finissais mon travail, j'allais ranger un peu...

Ces questions d'encad rement absent ou défaillant renvoient très directement à celle du statut d'apprenti.e, notamment à l'ambiguïté fondatrice entre apprentissage et production (G. Moreau, 2000). Cette tension entre deux logiques antagonistes sera développée plus loin.

Par ailleurs, certain.e.s jeunes déplorent un encadrement de mauvaise qualité. Cela peut être lié à divers facteurs: la personne formatrice n'a pas ou plus les compétences professionnelles requises, elle ne maîtrise pas les compétences pédagogiques indispensables à la transmission du métier ou encore elle n'a pas la volonté de former un·e apprenti.e. Voici ce qu'affirme à ce sujet Oriane, une spécialiste en restauration de 16 ans :

Mon patron, il me regardaitgenre: «Mais, en fait, je vois pas...», enfin, en m’engueulant «Ouais, mais je vois pas pourquoi tu 
vas [aux cours interentreprises], vous avez une école, ça suffit largement, un jour par semaine, et vous apprenez quoi ?» Et pis, moi, ça m’a fait un déclic, parce que j'étais là: «Ah, mais bon euh, enfin, c'est plutôt à lui de, de savoir ce que je dois apprendre.»

[...] C’est là que je me suis dit, je me suis quand même posé des questions, comment, enfin: "Est-ce qu'il a pas reçu les brochures, est-ce qu'il a lu les, euh ce que je devais apprendre?»

Souvent, les apprenti.e.s se plaignent du manque d'encouragements. Giovanna, une coiffeuse de 16 ans, déplore cette absence de reconnaissance :

Elle trouvait toujours des choses... je faisais tout le ménage, y avait un p'tit truc que j'avais pas fait, toujours quelque chose qu'allait pas.

[...] Aussi, avec une clientèle limitée, on peut pas apprendre, ouais. Y avait aussi qu'on était... manque d'encouragements, de félicitations.

Ceci pourrait être mis en lien avec le fait que les apprenti.e.s en début de formation sont particulièrement sensibles aux feedbacks sur la qualité de leur travail. En pleine phase de construction d'une identité professionnelle, elles et ils ont en effet besoin d'être conforté.e.s quant à leurs capacités et quant à leur choix professionnel. Dans ce cas, l'absence de reconnaissance se double d'une assignation au «sale boulot» (Hughes, 1996; Lhuilier, 2005), caractéristique du statut d'apprenti.e et d'une charge insuffisante de travail.

Une charge insuffisante de travail rend l'apprentissage du métier difficile: il n'y a pas de quoi apprendre et il faut gérer l'ennui. A cela s'ajoute le stress de ne pouvoir apprendre le métier au même rythme que les collègues de cours se formant dans d'autres entreprises. Adnan, un informaticien de 18 ans, l'exprime bien dans son témoignage:

Je sortais tout le temps, je sortais le plus possible pour aller parler, pour aller dans l'entreprise me balader, parce qu'il y avait rien à faire. Je me baladais, heureusement quel'entreprise me faisait marcher et tout, c'était le seul aspect qui était bien, quoi, ben, sinon, j'espérais que quelque chose tombe en panne.

[...] Dès qu'il y avait quelque chose en panne, c'était plutôt, ils faisaient appel à [YZ, une entreprise informatique externe], parce que c'était que des ordinateurs de chez [YZ] et puis 
c'est eux qui les réparaient. Moi, je les regardais comme ça, je disais: "Ça c'est ce que j'aimerais faire.»

L'un des enjeux principaux du système dual est l'apprentissage dans divers lieux de formation (école, entreprise, cours interentreprises). Dès lors, dans nombre de situations, un décalage peut exister entre les cours et la pratique (Dubs, 2006). L'école professionnelle joue ici un rôle prépondérant, puisque c'est dans ce cadre, en discutant avec les enseignant.e.s et surtout en comparant avec les collègues de cours, que l'apprenti.e prend conscience qu'elle ou il n'apprend pas correctement le métier sur son lieu de travail. Agnès, une peintre en carrosserie de 18 ans, en a fait l'expérience:

Surfacer, là, enfin, passer la surface, jel'ai fait trois fois, je crois. Tandis que ceux de ma classe, ben, ils avaient déjà tout fait.

[...] Moi, j'en ai parlé, en fait, aux cours aussi, et ils m'ont dit que c'était pas normal, parce que, malgré tout, les apprentis, on les utilise pas pour nettoyer les voitures ou comme ça, quoi.

[...] Tout ce qu'ils ont appris, aux cours, en fait, les personnes qui sont aux cours, ils ont tous déjà giclé, ils ont tous déjà fait quelque chose. Moi, à part nettoyer, rien du tout, quoi. C'est vrai que c'était pas facile.

Cet exemple témoigne non seulement du statut d'apprenti.e, une position subalterne et en charge des travaux rébarbatifs, notamment ceux d'entretien, mais il met encore en évidence la situation particulière d'une femme insérée dans un métier traditionnellement masculin. Ce second aspect sera repris plus en détail ultérieurement (voir notamment le Chapitre 8). Ces situations illustrent également le fait que les apprenti.e.s sont en train de faire aussi un véritable apprentissage du monde du travail. L'absence de vraies expériences professionnelles fait qu'elles ou ils n'ont pas encore de repères leur permettant de juger ce qu'il est usuel de faire dans l'entreprise. La confrontation avec leurs pairs fait ressortir les anomalies et leur permet de réagir. Plus globalement, ces cas de figure rappellent également un "point faible» du système de formation professionnelle duale, soit la difficulté qu'il peut y avoir à intégrer les enseignements scolaires et les apprentissages sur le lieu de travail (Dymock et Gerber, 2002).

Certaines situations s'apparentent à de véritables situations d'exploitation. Dans ces cas, la personne en apprentissage est soit déjà considérée comme un·e employé·e, qu'il n'est plus nécessaire de former, 
soit elle est utilisée comme main d'œuvre à bon marché et est assignée aux travaux les plus rébarbatifs ou dégradants. Adnan, un informaticien de 18 ans, rentre dans le premier cas de figure:

Je pense que j'étais carrément un employé, ils étaient même très contents et puis il y avait rien que je faisais comme quelqu'un qui apprenait, quoi. C'était vraiment, je faisais comme un travailleur, comme, ben, j'étais plus sur le lieu du travail que la personne qui m'apprenait, quoi. Je faisais plus de dépannages que lui.

A noter que lorsque l'apprenti.e est considéré.e comme un·e véritable employée, qui n'a besoin d'aucune formation, les tâches qui lui sont attribué.e.s s'en ressentent, mais pas forcément son intégration et son statut au sein de l'entreprise. Les paroles de Sarah, une fleuriste de 16 ans, sont très éclairantes à ce sujet:

En fait, je faisais tout, j'étais un esclave! [...] Mais, au début il m'apprenait vachement bien les trucs, on apprend beaucoup de choses avec, mais on apprend des choses qu'on devrait pas apprendre, pour que ça lui évite à lui de le faire.

Il y a dans ce cas, une vraie utilisation de l'apprentie pour se décharger $\mathrm{du}$ «sale boulot». Non seulement, elle fait tout comme une employée, mais encore elle fait ce que son employeur, ainsi que la seconde employée (l'épouse de l'employeur) ne veulent pas faire. L'assignation au travail ingrat s'inscrit dans un contexte d'organisation du travail qui repose sur une division, notamment sexuelle du travail.

Cette assignation quasi systématique aux tâches d'entretien, qui vaut aussi pour les apprentis, souligne que l'organisation du travail se construit également sur les bases de la division sexuelle du travail (Renard, 2015). C’est ainsi que certains garçons, assignés eux aussi à des tâches de ménage et d'entretien par leur statut d'apprentis, endossent, provisoirement en tout cas, du "genre féminin » (voir Chapitre 8). Agusto, un dessinateur en bâtiment de 18 ans, est par exemple concerné par cette mobilité de genre involontaire (Daune-Richard et Devreux, 1992; Guichard-Claudic et al., 2008) :

En fait, il [l'architecte] a pas tellement respecté sa part du contrat, parce que normalement ils sont censés nous apprendre le métier, pis j'ai fait peut-être trois ou quatre dessins pendant l'année, pis sinon, tout le reste, c'était corriger les soumissions ou bien faire le ménage. 
Le fait d'endosser - parl'assignation à certaines tâches - du genre féminin est vécue comme un véritable déclassement ${ }^{69}$, et ce plus particulièrement par les garçons. Dans la citation du jeune homme ci-dessus, cette mobilité descendante est également restituée en termes de rupture du « contrat » de formation. Le manque (de formation, d'accès au métier) est ainsi renvoyé à un rapport salarial, dans lequel les garçons s'inscrivent fréquemment (G. Moreau, 2000).

Enfin, dans certains cas, les mauvaises conditions de formation et les situations d'exploitation deviennent proprement insupportables pour les apprenti.e.s. C'est ce qu'exprime Jeremy, un gestionnaire de commerce de détail de 20 ans :

Il [le patron] sait même pas ce que c'est que de former un apprenti! Il croit que je suis juste là pour l'aider! En fait, je suis un bouche-trou, pour lui! Voilà, c'est ce qu'il a dit!

[...] Bien sûr, il l'a dit, que j'étais un bouche-trou! Pis, heu... ça le faisait chier de, comme il a dit, ça le faisait chier de payer des cours interentreprises, puis c'est depuis là qu'il voulait me virer!

[...] Il me disait: «bouche-trou!» Il disait devant moi, devant les autres: «Ouais, t'es un bouche-trou!» L'envie de le taper, ça me démangeait!

Ces résultats semblent confirmer la part importante que jouent les conditions de formation, associées à la qualité des relations au sein de l'entreprise, non seulement pour la satisfaction et le bien-être (en termes de santé mentale) des apprenti.e.s, mais aussi pour espérer une issue favorable à leur apprentissage. D'autres études (Kaiser et al., 2007) montrent en effet que ces éléments sont parfois même presque plus importants que les contenus du métier.

Ces éléments autour des conditions de formation mettent également en évidence les tensions, voire les contradictions liées au statut d'apprenti.e. Un statut ambigu entre apprenant.e et employée, entre personne en formation et personne ayant déjà une part active dans la production. Ce flou dans le statut d'apprenti.e explique en partie que la part formative de l'apprentissage dual disparaisse parfois quasiment. En effet, l'«employé·e» est censé·e déjà connaître le métier et n’a donc

69 Le terme déclassement permet de faire apparaître la mobilité descendante d'une classe de sexe vers l'autre. Ailleurs, et en guise de synonyme la notion de mobilité descendante sera convoquée. 
plus besoin d'être formé.e, tandis que la main d'œuvre bon marché fait faire le travail avec une contrepartie minimale, que ce soit en termes de salaire ou encore de formation.

\subsection{Des relations de travail difficiles}

Plus de la moitié des jeunes interviewé.e.s indique de mauvaises relations au travail $(\mathrm{N}=27)$; pour deux personnes, cela constitue même l'unique motif de la résiliation du contrat. Dans l'ensemble, l'arrêt de formation est lié à des problèmes que l'apprenti.e a rencontrés dans ses interactions au sein de l'entreprise formatrice ${ }^{70}$. Ces situations sont assez hétérogènes et peuvent être situées, d'après leur gravité et leur ampleur, sur un continuum allant de la mésentente avec un membre de l'entreprise à des situations de dysfonctionnement. Entre ces deux pôles interviennent d'autres cas de figure: des problèmes de climat de travail, ainsi que des interactions difficiles expliquées par le statut particulier des apprenti.e.s au sein de l'entreprise. L'acception du terme «relationnel» se veut donc très large, puisqu'elle comprend aussi bien des interactions déplaisantes (mésentente et climat de travail) que des interactions illustrant de véritables dysfonctionnements (harcèlement moral et sexuel, discrimination). Cette définition permet d'inclure des problèmes de type interindividuel (problème entre apprenti.e et collègue) et d'autres relevant de l'organisation du travail (problèmes liés à des positions hiérarchiques, notamment celle particulièrement vulnérable d'apprenti.e). Il a été difficile d'établir les contours de cette catégorie, et l'idée d'un continuum s'est imposée. Elle permet de ne pas séparer arbitrairement les différents aspects de la problématique relationnelle entre problèmes interpersonnels et conditions de travail, car les deux

70 Comme évoqué précédemment pour les conditions de formation, les questions relationnelles renvoient avant tout à l'entreprise et non à l'école professionnelle. Bien que durant l'entretien, le volet scolaire de la formation duale ait également été abordé, les jeunes ont surtout présenté dans leurs propos leur expérience en entreprise. Si la nouveauté de l'expérience peut expliquer en partie cette prépondérance de l'entreprise, elle souligne également l'importance, pour les jeunes qui choisissent la formation professionnelle duale, de l'entrée sur le marché du travail. La faible évocation de l'école professionnelle met enfin en exergue un autre aspect: l'absence de collectifs de pair.e.s. En effet, les cours professionnels sont l'une des seules occasions où les apprenti.e.s peuvent échanger leurs expériences entre «collègues». Or, la fréquence des cours (un jour à un jour et demi par semaine) et l'importance relative que leur accordent les jeunes ne permettent pas à de tels collectifs d'exister. 
peuvent être liés, et inscrire en continuité les questions structurelles (harcèlement psychologique ou sexuel). Cela permet également de rester au plus près de la raison, telle qu'évoquée par les apprenti.e.s.

La question relationnelle semble jouer un rôle déterminant dans le processus conduisant à l'arrêt prématuré, qu'elle apparaisse comme cause unique ou associée à d'autres raisons (notamment l'apprentissage du métier et les difficultés liées au monde du travail).

\subsubsection{Des tensions individualisées}

Certain.e.s jeunes évoquent un problème d'entente avec un.e collègue ou avecun.e supérieur.e hiérarchique. Les questions de statut et de hiérarchie sont toujours présentes en filigrane dans leurs témoignages, mais l'on peut considérer que dans certaines situations, la problématique est avant tout de type interpersonnel. C'est le cas de Célia, une coiffeuse de 18 ans:

Au mois de janvier, j'avais eu des problèmes avec ma patronne, parce que ça allait pas très bien, je lui répondais, pis on s'entendait pas très bien.

[...] C'était après les fêtes, tout le monde était sur les nerfs, on en avait tous marre et pis ça allait pas très bien au salon, alors on s'était mis d'accord qu'on allait faire des efforts, qu'on allait essayer de, moi moins répondre et pis elle, en fait, de moins m'agresser, parce que c'est vrai que c'est une femme assez agressive envers les gens. Pis ben, je me suis dite que j'allais essayer, que j'allais rester là-bas, pis ben du coup ça s'est pas amélioré.

Ce premier cas de figure, bien qu'il mette en scène une patronne et son apprentie, n'est pas présenté par la jeune femme comme un problème de hiérarchie ou d'organisation du travail, mais comme une mésentente. La résolution proposée étant d'ailleurs de faire des efforts de part et d'autre.

Quatre ex-apprenties affirment en outre que des problèmes interindividuels sont apparus lorsque la personne responsable de la formation en entreprise a commencé à s'immiscer dans leur vie privée. Ce comportement semble s'apparenter à une forme de paternalisme exercé par certain.e.s employeur.e.s. Cette attitude conforte explicitement la hiérarchie de genre et il n'est guère surprenant qu'il s'exerce ici exclusivement sur des filles. C'est le cas de Sylvie, une spécialiste en restauration de 16 ans: 
Ce que j'accepte pas, c'est qu'après mon travail, il [le patron] s'occupe de mes affaires privées. Déjà avec les fréquentations que j'ai, où je vais l'après-midi, ce que je fais quand je suis censée avoir congé [...] J’vois pas pourquoi on devrait me juger sur ma vie privée au travail!

[...] Il a dit qu'il voulait me protéger! Mais moi, non, je suis pas sa fille! Je suis quelqu'un qui vient travailler, qui vient faire un apprentissage. Ça n’a rien à voir.

[...] Il mélangeait le privé et le professionnel! Et ça, moi... dans le monde du travail, j'accepte pas. Moi, je sépare bien ce genre de choses.

Ce cas de figure souligne aussi une ambiguïté supplémentaire du statut d'apprenti.e, en l'occurrence d'apprentie. Dans certaines situations, notamment en formation atypique, les apprenties sont considérées comme des mineures ${ }^{71}$. Certains formateurs ou employeurs développent alors des formes de paternalisme, dans le but de protéger les jeunes femmes. Cette surprotection est d'ailleurs fréquemment dénoncée par les pionnières qui rejettent des attitudes qu'elles jugent stigmatisantes (Dallera et Ducret, 2004). Ces situations illustrent par ailleurs la complexité du rôle de formateur (Masdonati et Lamamra, 2009). En effet, outre un professionnel de référence, la personne agit aussi fréquemment comme un adulte, voire un parent. Ce faisant, l'employeur, par exemple celui évoqué ci-dessus, sort de son rôle professionnel et fait montre d'une représentation très hiérarchisée et stéréotypées des rapports de sexe, les femmes devant être contrôlées et protégées. Il incarne par contre parfaitement son «rôle» d'homme, protecteur et paternaliste.

Enfin, ces témoignages montrent que les apprenties tiennent particulièrement à séparer les différentes sphères de leur vie. Refuser l'intrusion dans la sphère privée, c'est aussi signifier qu'on aimerait être considérée comme une professionnelle, en tout cas en devenir.

Dans d'autres situations, les problèmes relationnels laissent davantage émerger les enjeux de pouvoir et de rapports de force soit entre l'apprenti.e et ses collègues, soit entre l'apprenti.e et son employeur.e. C'est le cas, par exemple, de Laetitia, employée de commerce de 16 ans, pour laquelle le rapport hiérarchique se joue avec l'apprenti de $3^{e}$ année:

71 De fait, certain·e-s le sont légalement. L’entrée en apprentissage peut se faire dès l'âge de 15 ans. 
Mais, je veux dire, la manière qu'il [l'apprenti de $3^{\mathrm{e}}$ année] m'a dit ça [de ranger], il m’a dit un peu, euh : "C'est moi le boss et pis c'est toi la bonne.»Pis moi j'ai fait: «Ecoute, tu ne parles pas comme ça!» C'est genre, moi je fais volontiers, vu que ce n'était pas, que je travaillais pour là-bas, mais qu'on me parle gentiment aussi, voilà. Pis, en fait, c'était une prise de tête.

Cet extrait met en évidence qu'une mésentente peut s'expliquer par une différence hiérarchique et la division du travail qui y est associée. Or, au bas de l'échelle, les apprenti.e.s sont particulièrement vulnérables et susceptibles d'être abusé.e.s par l'ensemble des personnes qui travaillent à leurs côtés : supérieur.e-s hiérarchiques, collègues, voire apprenti-e.s plus âgé.e.s. Le cas présenté ici illustre qu'il existe une hiérarchie, fondée sur une organisation et une division (sexuelle) du travail, entre apprenti.e.s également.

D'autres analyses (Masdonati et Lamamra, 2009) ont montré un lien étroit entre relations au travail et transmissions des savoirs professionnels. Il est donc probable que les problèmes rencontrés avec la personne chargée de leur formation aient pour conséquence des difficultés de formation. Cela se devine dans le récit de Yasmine, une assistante dentaire de 16 ans, qui est bloquée dans son apprentissage par la peur que lui inspire son patron:

Une fois, il [le patron, dentiste] m'a demandé d'aller chercher de la cire pour les dents, pour le détartrage. Pis je savais pas où elle était, pis j’ai demandé d'aller à une apprentie, elle m’a dit: «Débrouille-toi.» Donc j’ai fait long à la trouver. Pis après ben, il a gueulé parce que j’avais fait trop long. Enfin, trop long, j'veux dire, 10 minutes [...] Il a monstre gueulé [...] pis il a dit: «Tu sors tout de suite maintenant!»

Dans ce témoignage, la jeune femme n'ose pas dire qu'elle ne sait pas où se trouve le matériel, de peur d'être réprimandée par son patron. Elle ne peut donc pas apprendre les gestes techniques de sa profession en toute sérénité, elle n’a pas accès aux «trucs du métier» (Filliettaz et al., 2008). Comme plus haut, cette situation ne découle pas seulement des rapports hiérarchiques avec les employeur.e.s, mais aussi avec d'autres apprenti.e.s. Dansl'extrait cité, l'autre apprentie se positionne par rapport à sa collègue de première année sur un mode compétitif. Ainsi, le rapport de pouvoir institué par la position particulière de l'apprenti.e, en particulier par les apprenti.e.s de première année, se répercute dans toutes les relations de travail au sein de l'entreprise. Cette asymétrie, instaurée par l'employeur 
et agissant dans la relation entre apprenti.e.s, est également soulignée par Leonora, 16 ans, elle-aussi assistante dentaire:

L'apprentie de $3^{\mathrm{e}}$ année, c'est avec elle que j'ai eu le plus de problèmes [...] Il [le patron, dentiste] m'a juste dit qu'il fallait que j'écoute l'apprentie, ce qu'elle me disait, qu'il fallait que je sois sous ses ordres comme pour ma diplômée [formatrice assistante dentaire]

[...] Elle m’a traitée de «bête», d'incapable. Si c'est moi qui devais aller faire les courses, elle me disait: «Non, parce que toi tu viens d'arriver, pis t’as déjà le droit de sortir, ben non, c'est moi qui irai, tu devras rester...»

Cet extrait met également l'accent sur l'organisation du travail, les tâches subalternes semblant être «réservées » aux nouveaux et nouvelles arrivant.e.s. La division du travail qui traverse l'entreprise, hiérarchise donc également les apprenti.e.s en fonction de leur année d’apprentissage et organise leur travail selon ce même critère. Compte tenu des tâches auxquelles les apprenti.e.s de première année sont assigné.e.s, cette hiérarchie entre les apprenti.e.s se fonde sur la division sexuelle du travail, ou plutôt l'une des «nouvelles modalités de la division sexuelle du travail» agissant également entre femmes (Hirata et Kergoat, 2010) ou entre personnes ayant un statut dominé ${ }^{72}$. Ainsi, dans l'exemple précité, l'apprentie - ayant par son ancienneté un statut légèrement supérieur délègue des tâches à sa collègue au statut plus fragile ou encore la restreint dans sa liberté de mouvement.

72 Dans leur article Hirata et Kergoat (2010) examinent les nouvelles modalités de la division sexuelle du travail. Depuis le début des années 80 , on assiste simultanément à une augmentation des femmes cadres ou occupant des professions intellectuelles supérieures et une précarisation croissante de femmes cantonnées dans des emplois subalternes. «On voit ainsi apparaître, pour la première fois dans l'histoire du capitalisme, une couche de femmes dont les intérêts directs (non médiés comme auparavant par les hommes: père, époux, amant...) s'opposent frontalement aux intérêts de celles touchées par la généralisation du temps partiel, les emplois de service très mal rétribués et non reconnus socialement, et plus généralement par la précarité » (Hirata et Kergoat, 2010:4). En outre, les femmes du Nord travaillent de plus en plus, et comme le travail domestique n'est toujours pas pris en compte dans nos sociétés, elles doivent externaliser «leur» travail domestique. Pour cela, elles recourent au réservoir de femmes précaires-pauvres, européennes ou immigrées. On assiste donc à de nouveaux rapports sociaux entre femmes, inédits historiquement, dont un rapport de classe entre les femmes du Nord, employeuses, et cette nouvelle classe servile. 
Ces problèmes hiérarchiques inter-apprenti.e.s se déclinent de diverses manières, et si aucun jeune homme ne mentionne ici de problèmes liés à la question de l'ancienneté, certains mettent en évidence des hiérarchies qui peuvent exister entre différentes formations. C'est le cas de Matthieu, un vendeur en pièces détachées de 16 ans, qui relève que son formateur s'entend mieux avec les apprentis mécaniciens. Il laisse ainsi entendre que, dans un garage, il y a deux univers distincts. La hiérarchie s'organise dans ce cadre entre cols-blancs et cols-bleus, mais pas dans le rapport auquel on pourrait s'attendre. En effet, bien que le statut d'employée soit socialement considéré comme supérieur à celui d'ouvrière ou d'ouvrier (Joye, Bergman et Budowski, 2007), il n'est pas reconnu dans cet univers «viril». On pourrait presque parler d'inversion des valeurs : le travail manuel étant plus valorisé que le travail intellectuel. Le travailleur manuel, qui se salit au contact des moteurs et use de sa force, obtient ici davantage de "prestige » que l'employé, le vendeur, au contact de la clientèle. Ce processus d'inversion des valeurs relève d'une forme de résistance virile (Dejours, 2000b; Molinier, 2004). Afin de revaloriser un secteur professionnel peu reconnu, ce système défensif propose une hiérarchie des activités qui se calque sur une grille virilité/féminité (Molinier, 2000): l'activité associée au masculin (la mécanique automobile) est placée dans un rapport de supériorité avec l'activité associée au féminin (la vente) et ce indépendamment de la reconnaissance sociale de chacune de ces activités.

\subsubsection{Le climat de travail}

Dans d'autres cas, les problèmes de relation sont collectifs (et impliquent plusieurs collègues). Lorsque les problèmes se posent au sein de l'équipe, ils peuvent aboutir à des difficultés d'intégration de l'apprenti.e. Les jeunes, déçu·e.s par l'ambiance qui règne au sein de l'entreprise, jugent leur situation insupportable. C'est le cas pour Anaëlle, une horticultrice de 18 ans:

C'est vraiment une ambiance de... au début, on se rend pas compte, parce qu'on connaît pas, aussi, mais après, on se rend compte que c'est vraiment un monde, euh, comment on dit, si je peux dire: faux-cul! Tu dis quelque chose à quelqu'un, par derrière il va dire autre chose à une autre, qui va dire autre chose à une autre, pis... en fait, toute l'entreprise le sait, pour finir tout est déformé et pis, après, on... enfin, c'est vraiment... Tu vas dans une équipe, ils disent ça sur une autre, tu vas 
dans une autre, ils te disent ça sur... enfin, c'est vraiment, euh, c'est pas facile!

Un climat de travail délétère est parfois le fait de groupes de collègues fonctionnant comme de véritables clans et face auxquels il s'agit de se positionner, ce qui est particulièrement difficile pour un·e apprenti.e dont c'est la première expérience dans le monde du travail. Ces situations illustrent le climat de concurrence généralisée qui peut régner entre les collègues et la fragilité de la position d'apprenti.e. Aurélie, une gardienne d'animaux de 17 ans, en donne un bon aperçu:

Il y avait des groupes qui se formaient, alors si tu voulais t'entendre avec cette personne, par exemple, imaginons vous êtes ma collègue de travail, si je veux m'entendre avec vous, OK on s'entend, on s'entend très bien... Mais par exemple, si vous, vous vous entendez pas bien avec ce collègue là-bas, mais moi oui, alors moi je dois plus m'entendre avec celui-là si je veux rester avec vous. Y avait tout des trucs comme ça: «Toi, je t'aime bien donc je vais t'apprendre à faire ça; toi, je t'aime pas trop alors on va attendre un peu; toi, je t'aime pas du tout, alors tu fais rien quoi!»

La jeune femme met bien en évidencele lien entre relation et transmission des savoirs. Ainsi, outre la difficulté à travailler dans un environnement marqué par les concurrences et les conflits entre collègues, l'accès à la formation peut lui être refusé si elle ne fait pas les bonnes alliances.

Le mauvais climat au sein de l'entreprise crée parfois des désillusions chez les apprenti·e.s, le monde du travail ne répondant pas aux représentations qu'elles ou ils s'en faisaient. Ce désenchantement peut conduire à des formes de souffrance éthique (voir Chapitre 6). Cette déception se perçoit bien chez Daniele, un opticien de 17 ans :

Le problème c'était que... c'était même... c’était les employés qui s'occupaient de moi, avec l'employeuse ça allait pas du tout, mais y avait un gros problème, c'est que dans cette équipe-là ils se critiquaient tous, alors si déjà entre eux ça va pas... forcément ça ira pas avec moi!

Je trouve ça dommage, parce que si tu aimes ce que tu fais mais si y a des gens, parce que toi aussi t'as une partie de faute, mais c'est eux qui te donnent cette étincelle, s'ils te la donnent pas, ça peut pas aller. 
Cet extrait montre un élément récurrent dansles témoignages des jeunes en arrêt de formation : la question de l'ambiance, du climat de travail. L'aspect relationnel sous sa forme collective a été maintes fois identifié dans la littérature (Cohen-Scali, 2003; Eckmann-Saillant et al., 1994; Gauthier, 1994; G. Moreau, 2003). Cet aspect prime parfois sur les autres critères d'appréciation de la formation, y compris sur l'intérêt intrinsèque pour le métier (Meeus, Dekovic et Iedema, 1997). Comme si ce qui comptait, c'était de se former dans une équipe accueillante, qui intègre et qui procure du plaisir sur le lieu de travail, indépendamment du type de tâches effectuées.

L'ambiance de travail n'est que très épisodiquement évoquée par les filles, mais revient dans la plupart des témoignages masculins. On pourrait faire l'hypothèse que les filles se mettent dans des rapports de travail moins concurrentiels que les garçons, ayant appris au cours de leur socialisation à développer les relations de collaboration (Dafflon-Novelle, 2006; Hurtig, 1998; Mosconi, 1994), pendant que les garçons ont appris durant la même période les logiques de concurrence (Zaidman, 1996). Par ailleurs, les garçons investissant plus largement le volet «travail» de la formation duale (G. Moreau, 2000), il n'est guère étonnant qu'ils en relèvent l'ambiance. Enfin, comme cela a été illustré précédemment, les filles connaissent d'autres types de problèmes relationnels, l'intrusion dans leur sphère personnelle, notamment. La question de l'ambiance de travail apparaît donc fréquemment chez les ga rçons, comme par exemple chez Andrew, un ferblantier de 17 ans:

C'est la sale ambiance que j'ai avec les employés... mes collègues. Et le matin, arriver à 7 heures et pis partir à 7 h30 sur le chantier, t'es là en hiver et pis il fait -5 dehors, non!

Le jeune homme en fait un facteur déterminant de son arrêt prématuré. Il souligne la centralité du climat de travail, qu'il met dans la balance avec la pénibilité du métier. Il montre ainsi qu'il peut y avoir une «relation dialectique [...] entre l'ambiance et les conditions de travail, la première fonctionnant comme un antidote fondamental aux secondes» (G. Moreau, 2000 : 215). Quand l'ambiance n'est pas bonne, que les conditions de travail soient pénibles ou non, mais d'autant plus lorsque elles le sont, le maintien en formation devient impossible.

\subsubsection{Le harcèlement}

Dans certaines situations, les limites de l'acceptable sont franchies. Certains récits sont en effet accablants, à tel point que la notion de har- 
cèlement psychologique semble tout à fait adaptée. D'après le Secrétariat d'Etat à l'Economie, ce comportement, aussi appelé mobbing, consiste en «l'enchaînement, sur une longue période, de propos et/ou d'agissements hostiles, exprimés ou manifestés par une ou plusieurs personnes envers une tierce personne transformée en cible. Le mobbing concerne dans la plupart des cas une seule personne jouant tôt ou tard le rôle de bouc émissaire» (SECO, 2005). Ces agissements peuvent toucher la personnalité, la dignité ou l'intégrité d'une personne. Ils menacent ainsi l'emploi de la victime et contribuent à dégrader le climat de travail (Hirigoyen, 1998). Les propos et agissements en question sont de quatre types: des actes de persécution par le biais de mesures organisationnelles, des actes visant l'isolement social de la victime, des actes mettant en danger son intégrité physique et des actes dirigés contre son intégrité psychique et sa personnalité. Le harcèlement ${ }^{73}$ concerne près d'un tiers des situations décrites par les jeunes interviewé.e.s. Dans ces cas, on est donc largement au-delà de simples problèmes d'entente ou de conflits ponctuels avec un.e ou des collègues ou supérieur.e.s, mais plutôt dans des modes d'interaction dysfonctionnels correspondant à la définition évoquée ci-dessus.

Un exemple, mettant en danger l'intégrité psychique et la personnalité de la victime, est donné par Leonora, une assistante dentaire de 16 ans :

Elle [une apprentie en troisième année] était toujours un peu derrière moi ou qui me disait: «T’as pas le droit de faire comme ta diplômée [une autre collègue], parce que t'es incapable», des choses comme ça, et je perdais un peu de confiance en moi.

[...] J'avais toujours l'enthousiasme de travailler, mais le seul problème c'est quand j'ouvrais la porte du cabinet, c'était «je vais croiser [XY], pis je me demande ce qu'elle va me dire de travers ...». J'avais quand même une sorte de peur envers elle.

[...] Pis elle est venue vers moi, pis elle m’a dit: «Prends-le pas mal, mais tu pues. " Après, elle est allée parler de ça dans le cabinet, avec euh [l'autre collègue], et elles se sont enfermées, elles ont fermé la porte pis, euh, ben, j'étais en larmes.

73 Comme le SECO, les deux termes «harcèlement psychologique » et «mobbing» sont utilisés indifféremment ici. 
Le harcèlement prend parfois aussi la forme d'une mise à l'écart organisée par plusieurs membres de l'équipe de travail. Sylvie, spécialiste en restauration de 16 ans, en donne un bon exemple:

Je rentrais du travail, je pleurais, parce que je me sentais mal. En plus, bon ben, j'avais une caisse, donc il me manquait tout le temps de l'argent dans ma caisse, soi-disant que c'était de ma faute, mais à plusieurs reprises j'ai vu mes collègues carrément des fois déchirer des tickets devant moi en disant: "Ah, ça c'est à moi, est-ce que tu peux me redonner l'argent?» Donc, voilà, des fois j'avais l'impression d'être mise un peu à l'écart et pis euh, d'être le bouc émissaire de tout le monde.

Certains récits révèlent l'existence parallèle de plusieurs types de harcèlement. Alexandra, une cuisinière de 22 ans, affirme être en même temps isolée socialement et menacée physiquement:

Y a eu des insultes, des menaces, y'a eu euh... des bousculades, c'était une pression très forte.

[...] Une menace, c'est: «Je vais t'en coller une, tu vas t'en prendre une, tu vas être contente ! », ça, c'est une menace ! C'est me prendre, me bousculer, pis: «Je vais t'éclater!»C'était, ça c'est une menace!

[...] Je crois quej'ai toujours été, de toute façon, ils m’appelaient «l'apprentie». J'ai jamais été vraiment intégrée. Y'en a qui me considéraient vraiment comme leur collègue et puis d'autres, ils me disaient vraiment: «Moi, c'est moi et puis toi... toi... t'es l'apprentie!»

Ces cas de mobbing sont plus évoqués par les jeunes femmes que par les jeunes hommes. Ils rendent compte de la violence sur le lieu de travail, qu'elle soit exercée par un·e supérieur·e, un·e collègue ou par l'ensemble du collectif de travail. Ces situations croisent parfois d'autres problématiques, en particulier celle de l'insertion des jeunes femmes dans des professions largement masculinisées. Dans ces cas, le harcèlement moral semble faire partie des diverses stratégies mises en œuvre par les travailleurs pour protéger leur champ de l'entrée des femmes, qu'ils semblent vivre comme une intrusion (Eckert, 2007; Guichard-Claudic et al., 2008; Molinier, 2000), ce qui apparaît très nettement dans le témoignage d'Agnès, peintre en carrosserie de 18 ans: 
J'suis restée calme, parce qu'il faut être calme avec eux, parce que si on s'énerve, c'est ce qu' ils veulent. Ils m’ont dit : «On va te pousser à bout jusqu'à quand tu arrêtesl'apprentissage!» [...]

Le but ici est clairement de dégoûter la jeune femme de la profession, de se débarrasser de l'«intruse». Dans certains cas, les remarques, les humiliations ne suffisant pas, la violence physique prend place. Julie, une paysagiste de 16 ans, l'apprend à ses dépens. Ses collègues scient en effet la branche sur laquelle elle est assise, la faisant tomber. Suite à cet événement, elle sera en outre sanctionnée:

Il [son supérieur, à qui elle s'est plainte] trouvait: «Non de toute façon, t'avais pas à te mettre là.» Après, j'avais plus le droit non plus de grimper aux arbres, pour les tailler. [...] Avant je pouvais, hein. Maintenant, non: «T'as plus le droit! T'as plus le droit de monter sur les machines.» [...] Il lui a dit à son collègue: «J’veux plus la voir sur une seule machine.»

Non seulement la jeune femme est victime de violence, mais encore la sanction - ou faut-il y voir une forme de protection? - l'empêche de se former correctement. Tant le harcèlement que la sanction ont pour conséquence de l'empêcher d'accomplir certaines tâches et d'utiliser les machines, soit le cœur de l'activité professionnelle. Sur un autre plan, cette mise àl'écart signifie le non-accès au pouvoir, que permet la maîtrise des outils (Tabet, 1979). Ce faisant les hommes gardent la mainmise sur les outils de travail et le pouvoir qu'ils confèrent. La logique de contrôle des outils de production, ainsi que celle de protection/sanction reposent sur la division sexuelle du travail et participent à la construire (Malochet, 2008; Tabet, 1979).

Le harcèlement sexuel est une dernière forme de harcèlement qui concerne, dans ce corpus, exclusivement les femmes. Il peut être défini comme suit: «tout comportement importun de caractère sexuel ou tout autre comportement fondé sur l'appartenance sexuelle, qui porte atteinte à la dignité de la personne sur son lieu de travail, en particulier le fait de proférer des menaces, de promettre des avantages, d'imposer des contraintes ou d'exercer des pressions de toute nature sur une personne en vue d'obtenir d'elle des faveurs de nature sexuelle ${ }^{74}$. Il peut être le fait de collègues ou de supérieurs hiérarchiques et se manifester de diverses manières: par un environnement hostile, du chantage, des remarques et

74 Définition tirée de l'article 4 de la Loi fédérale sur l'égalité entre femmes et hommes (LEg), entrée en vigueur le $1^{\text {er }}$ juillet 1996. 
plaisanteries à connotation sexiste ou des agressions physiques (Ducret, 2008). Julie, une paysagiste de 16 ans, le relate ainsi:

Le matin, j'arrive pis il me fait: «Ouais, j’ai rêvé de toi toute la nuit », ou comme ça. Une fois je lui ai demandé, ça c'est un truc, ça m’est resté sur la tête, je lui ai dit: «En fait, c'est quoi le programme aujourd'hui? » Il me fait: «On va finir cette terrasse, après je te fais l'amour sur la table et après midi on recommence.»

[...] Aussi: «Ouais, hier j’ai vu à la télé une pub où y avait une femme qui léchait sa glace comme ça, pis ça m’a fait penser à toi.»

[...] Mon contremaître, ça lui est déjà arrivé, quand je bossais, en train de faire quelque chose, ben, une claque sur les fesses.

$[\ldots]$

Pis aussi des p'tits trucs, les p'tits mots de tous les jours, comme quand il [le deuxième employeur] m'a commandé mes salopettes de travail, il me fait: «Ouais, j'vais te commander des pantalons en cuir. Ouais, t’as reçu tes pantalons en cuir?» Tout des trucs comme ça. Moi j'étais là, j’peux rien répondre. [...]

Lorsquel'apprentie en question dénonce ces actes, le harcèlement change de forme et se transforme en mobbing, exercé par plusieurs personnes, cela contribue à sa stigmatisation et vise sa mise à l'écart.

Le harcèlement sexuel concerne, dans ce corpus, plus particulièrement les jeunes femmes qui sont insérées dans des professions atypiques. Il semble donc avoir la même fonction de défense contre l'entrée des femmes que le harcèlement moral, évoqué précédemment. Il permet également aux hommes de rappeler la hiérarchie entre les sexes et la place assignée à chacun d'entre eux (Tabet, 1979). Le harcèlement sexuel dénie à l'apprentie son statut de professionnelle, elle n'est plus une personne en formation ou une employée, mais une femme sexualisée et à disposition. C'est son «être-femme» que l'on met en avant et non pas ses compétences professionnelles. La virilité se joue donc là aussi : en sexualisant les femmes, en les associant à l'univers de la pornographie, on ne leur octroie pas de statut professionnel (Chamberland et Paquin, 2008; Molinier, 2002b). Le harcèlement sexuel sur le lieu de travail est donc un rappel fait aux femmes de la position qu'elles occupent dans les rapports inégalitaires entre les sexes. Le statut de pionnière amplifie 
encore le phénomène, les jeunes femmes cherchant à forcer l'entrée de «bastions masculins». (Chamberland et Paquin, 2008; Marry, 2007).

\subsubsection{Le statut d'apprenti-e}

Les questions relationnelles mettent en relief la question du statut d'apprenti.e: sa position subalterne, son absence de marge de manœuvre et de possibilité de résister. En effet, fréquemment isolé.e-s dans l'entreprise (seul.e.s apprenti.e.s entouré.e.s de collègues expérimenté.e.s et plus âgé.e.s), elles et ils ne connaissent en outre pas de solidarité auprès des autres apprenti.e.s lorsqu'il y en a, puisque l'organisation du travail les hiérarchise selon leur ancienneté.

La question du statut est au cœur des griefs des garçons, qui même lors de problèmes relationnels, font apparaître en filigrane ce qui les lie à leurs collègues ou employeuree.s : le rapport salarial (G. Moreau, 2000). Les jeunes hommes s'inscrivent explicitement dans un rapport de travail et attendent de leur employeur.e que cela soit respecté. Quentin, un pâtissier de 19 ans, dénonce par exemple l'absence de respect dont il fait l'objet de la part de son patron :

Le patron il me faisait souvent des remarques négatives, il me disait souvent des trucs rabaissants [...] Toujours à guetter, à faire des remarques tout au long de la journée...

\section{$[\ldots]$}

Si c'est un apprentissage qui se passe normalement [...] avec un patron avec qui on s'entend normalement. Un patron qui enfin fasse le boulot en même temps, en même temps apprend le métier, qu'on nous montre les choses. Qu'il y ait un certain respect quand même entrel'apprenti et le patron. [...] Pis quand même vouloir formerl'apprenti, pas juste vouloir rentabiliser...

Ce jeune homme lie relations et transmission des savoirs, tout en dénonçant les problèmes d'exploitation auxquels il est confronté. Il montre que les savoir-faire professionnels ne peuvent pas être transmis dans un cadre où la relation avec la personne formatrice n'est pas «normale", qu'ainsi il n'aura pas accès aux «trucs du métier» et au collectif de travail, éventuellement à une communauté de pratique (Filliettaz et al., 2008; Masdonati et Lamamra, 2009).

Ce statut ambigu fait osciller l'apprenti.e entre personne en apprentissage et employé e participant à la production (G. Moreau, 2000, 2003). Ainsi, au sein de l'entreprise, il n'est pas toujours clair de 
savoir si l'apprenti.e est un.e employé.e à part entière ou si elle ou il sert de main d'œuvre bon marché. La question du statut transparaît dans nombre de témoignages, notamment ceux rapportant des situations de harcèlement. Dans une position hiérarchique faible, l'apprenti.e est davantage exposé.e, notamment à des formes d'isolement social. Il en est ainsi pour Daniele, opticien de 17 ans:

Elle [la patronne] disait «ouais, j'en ai marre, c'est moi qui dois m'occuper de toi!» Comme si c'était, ouais, comme si j'étais un fardeau!

[...] Y a une phrase qu'elle m'a dite, et là ça m’a fait tilt, elle m’a dit... moij'ai dit: «Maisj'aimerais bien que vous me fassiez un peu plus confiance, pour que moi je sois en confiance aussi, je veux dire...» et elle m’a fait: "Ouais, tu pourras jamais être, heu...», moi je lui ai dit: "J'aimerais bien être plus intégré dans l'équipe», et pis elle me fait: «Non, tu pourras jamais, parce que t'as pas de diplôme!» Alors pour moi, ça, ça veut tout dire! Pour moi, je suis vraiment de l'autre côté de la table. Ils ont fait un souper de boîte: j'ai pas été invité.

[...] Eux ils étaient devant [dans le magasin], et pis moi j'étais tout derrière, dans un petit machin tout pourri, entre guillemets!

On remarque donc que les difficultés au niveau de la relation et de l'intégration de l'apprenti.e sont souvent tributaires du statut que celle- ou celui-ci a au sein de l'entreprise. Cet aspect illustre la relation entre harcèlement et rapports de pouvoir. La question du statut d'apprenti.e est assez sensible et fréquemment évoquée par les jeunes. C'est le cas d'Agnès, une peintre en carrosserie de 18 ans:

Pour eux, j'existais pas, en fait. Ils m’ont dit: «Une apprentie, ça compte même pas. » Pour eux, ouais voilà quoi, j'étais une apprentie, ils m’ont dit: «Une apprentie? T'apprends rien du tout, donc tu comptes pas, t'es là pour le décor, pour faire joli, pour nous aider et pis voilà quoi!» C'était ça quoi, on était une fille, ben du coup c'est la «conchita». Ben eux, s'ils prennent des apprenti.e.s, c'est surtout pour le nettoyage quoi!

Dans ce cas précis, la question du statut d'apprenti.e est renforcé par les rapports sociaux de sexe. Les collègues de la jeune femme lui rappellent son statut doublement subalterne : apprentie et pionnière. Il est quasiment sûr qu'un apprenti peintre en carrosserie ne se serait pas vu signifier 
son inutilité de cette façon, notamment en étant assignée à des tâches d'entretien et renvoyé à sa fonction «décorative». Le statut d'apprenti.e est ici instrumentalisé au service d'attaques sexistes.

Ces mises à l'écart peuvent parfois avoir un impact allant au-delà de la simple résiliation de contrat. En effet, dans certains cas, cela contribue à dégoûter l'apprenti.e de la profession choisie, confirmant une fois encore l'importance de la dimension relationnelle dans le maintien en formation ou au travail. Aurélie, une gardienne d'animaux de 17 ans, est dans cette situation. Elle subit une exclusion qui l'éloigne d'un métier dont elle aime pourtant beaucoup les contenus:

Quand ils sortaient les chiens, ils en profitaient pour discuter et tout, et pis quand c'était moi qui aidais, ben personne discutait avec moi, sauf pour me donner ce qu'il y avait à faire. Evidemment!

[...] Y a tout qui me plaît dans le travail, ben bien sûr y a des choses que j'aime moins faire que d'autres mais qui me dérangent pas du tout! Mais heu... ils avaient réussi à me dégoûter du travail, donc quand je suis partie, je me posais la question si je voulais encore faire ça ou pas! Parce que ça me disait quasiment plus rien!

Par leur statut et par l'insertion fragile qui en découle, les apprenti.e.s se retrouvent donc souvent en situation de fragilité, de vulnérabilité et sont parfois exposé.e.s à des comportements de violence psychique. En situation pionnière notamment, on assiste à l'imbrication entre rapports sociaux de sexe et statut apprenti.

\subsubsection{Discriminations liées à l'origine}

Outre le statut, d'autres discriminations en vigueur sur le marché du travail contribuent à fragiliser encore davantage les apprenti.e.s: le sexe, comme cette étude le montre et la nationalité, qui offre une prise aux attaques racistes. Ces discriminations sont peu évoquées dans les témoignages, mais interviennent néanmoins à quelques reprises ${ }^{75}$. Divers travaux se sont intéressés aux préjugés, stéréotypes et discriminations à l'égard des jeunes d'origine étrangère (qu'elles ou ils soient primo-arrivant.e.s ou immigré.e.s de la deuxième ou troisième génération). Il ressort de ces études que les jeunes d'origine étrangère subissent discriminations

75 Les discriminations racistes sont relativement peu évoquées dans l'étude, principalement composée de jeunes d'origine suisse ou bi-nationale. 
à l'embauche (Fibbi et al., 2003; Imdorf, 2004, 2007b), préjugés et stéréotypes durant leur expérience en formation professionnelle (C. Moser et al., 2008; Pfister Giauque et Flamigni, 2010), qu'elles et ils ont une transition plus délicate que les jeunes Suisses-ses (Rastoldo et al., 2007) et enfin qu'on les retrouve davantage dans la population des jeunes ayant interrompu leur formation professionnelle prématurément (C. Moser et al., 2008; Rastoldo et al., 2009) ou des jeunes sans qualification (Eckmann-Saillant et al., 1994). L'étude LEVA (C. Moser et al., 2008) met en outre l'accent sur les difficultés spécifiques des jeunes issu.e.s de la migration, en particulier les discriminations institutionnelles ${ }^{76}$.

Des propos racistes sont parfois rapportés pour décrire l'ambiance de travail, l'environnement dans lequel se fait la formation. Nuño, un coiffeur de 18 ans a dû composer avec cette violence quotidienne:

Des fois ils faisaient des blagues sur les Portugais, parce que j'suis Portugais, mais non, c'était pas mal, j'le prenais pas trop mal.

Il est intéressant de voir à l'œuvre ici un processus de banalisation, et ce par la personne même qui subit ces "plaisanteries». Cette stratégie ressemble à «l'autocensure» dont font preuve les pionnières pour supporter le sexisme: il s'agit de laisser couler, de prendre sur soi (Dallera et Ducret, 2004). Relevons encore que cette minimisation a également été observée du côté des enseignant.e.s professionnel.le.s, que ce soit par rapport aux propos racistes ou sexistes (Pfister Giauque et Flamigni, 2010). Ce processus laisse apparaître l'enjeu principal pour les jeunes, qui est ici l'intégration dans un groupe, dans un collectif de travail en l'occurrence. C'est probablement ce qui explique pourquoi cette forme de discrimination apparaît très peu dans le corpus. Les préjugés racistes participent donc des mauvaises relations de travail et peuvent parfois conduire à la rupture du contrat. C'est ce qu'a vécu Leonora, une assistante dentaire de 16 ans :

Elle [une collègue] est $100 \%$ suisse et des fois c'est vrai que, avecl'hygiéniste, la stagiaire hygiéniste, elles me sortaient des propos: «Les Albanaises, elles ont la raie de côté », « vivement qu'y a les étrangers pour faire des emplois.»

76 Il s'agit des modalités d'entrée dans nombre de filières de la formation professionnelle. Celles-ci reposent en grande partie sur les compétences linguistiques, écartant de fait la plupart des primo-arrivant.e-s, quel que soit par ailleurs leur niveau scolaire. 
[...] C'est vrai que des fois, je faisais des fautes d'orthographe comme «noir», je le disais «noirte», mais on m'avait jamais corrigée pour ça. Pis là, c'est vrai, des fois, qu'ils ont un peu abusé, sur le coup, pis qu'ils ont carrément dit, y m’ont envoyé une lettre, pourquoi je me suis fait licencier, c'était parce que je savais pas parler français.

Dans cet extrait, les préjugés n'ont pas seulement entaché l'ambiance de travail, mais ont également contribué au licenciement de la jeune femme, pour ses supposées lacunes en français ${ }^{77}$. Le lien entre arrêt prématuré en formation et discriminations racistes n'est pas évident à démontrer, car outre la stratégie de banalisation évoquée ci-dessus, il est probablement difficile pour les jeunes d'avoir la certitude que les problèmes rencontrés, ou l'absence de soutien obtenu ont à voir avec leur nationalité. C'est le cas de Hakem, un réparateur automobile de 19 ans:

Ah, non je veux dire euh, par exemple mon patron il était suisse et mon commissaire aussi, je veux pas dire que tous les Suisses ils sont comme ça, mais de temps en temps... Déjà dans le bus, il y en a beaucoup ils aiment pas les étrangers et tout ça. $[\ldots]$

Et pis depuis là [une altercation dans le bus avec une personne âgée lui ayant tenu des propos xénophobes], il y a certains Suisses qui ne veulent pas les étrangers.

Le jeune homme pense que le patron ne lui a pas donné sa chance, que le commissaire ne l'a pas soutenu à cause de sa nationalité, mais rien de tel ne lui a été signifié. En outre, cette éventualité est d'une telle violence, que le jeune homme a de la peine à l'exprimer et a attendu la fin de l'entretien pour en parler.

Les discriminations liées à la nationalité ou au sexe fonctionnent selon la même logique de catégorisation et de hiérarchisation (Roux, Gianettoni et Perrin, 2006). Elles sont à l'œuvre non seulement dans les processus de recrutement pour l'obtention d'une place d'apprentissage, mais encore dans l'expérience de formation professionnelle, notamment lorsque les personnes se voient signifier qu'elles sont en situation de transgression. En effet, la division du travail ne s'organise pas seulement

77 Une réserve sur le motif de son licenciement s'impose. En effet, la jeune femme a fait toute sa scolarité en Suisse, a obtenu son certificat de fin de scolarité secondaire sans redoublement et a fait un préapprentissage avant d'entamer sa formation d'assistante-dentaire. 
sur la base du sexe (D. Kergoat, 2000), mais aussi de l'origine «imputée ou supposée» (Lada, 2008).

Comme déjà évoqué à plusieurs reprises, les relations au travail et l'apprentissage du métier sont étroitement liés. Il est en effet difficile de concevoir des situations d'apprentissage dans lesquelles la personne connaît des difficultés dans les relations avec ses collègues ou ses supérieur.e.s. Cela d'autant plus lorsqu'il y a harcèlement ou exploitation. En d'autres termes, des bonnes relations de travail sont une condition sine qua non pour un bon apprentissage du métier. Dans le système dual, où l'accès aux savoir-faire du métier, passe notamment par le formateur ou la formatrice en entreprise et par l'intégration dans un collectif de travail, de mauvaises relations ont un impact immédiat sur la transmission des savoirs (Masdonati et Lamamra, 2009). Par analogie, lorsque la qualité de la formation dispensée en entreprise est discutable, elle risque de compromettre les relations au travail. Ainsi, comme le souligne Moreau (2003: 208): "la satisfaction vis-à-vis de la formation délivrée par le maître d'apprentissage passe [...] par d'autres chemins, celui notamment de la bonne ambiance».

\subsection{Les contraintes du monde du travail}

Le troisième type de raisons d'arrêt se rapporte aux contraintes caractéristiques du monde du travail. Cela concerne la moitié des jeunes interviewé.e.s $(\mathrm{N}=23)$. Leurs difficultés sont liées au fait que la formation a lieu essentiellement en entreprise, dans une organisation productive.

Les situations étant très hétérogènes, elles sont organisées en deux catégories: la première se réfère à de mauvaises conditions de travail, dues soit au métier choisi, soit aux conditions particulières rencontrées au sein de l'entreprise formatrice; la deuxième concerne des problèmes de santé au travail. Ces raisons interviennent la plupart du temps conjointement à d'autres difficultés (apprentissage du métier et relations de travail).

\subsubsection{Les conditions de travail}

La pénibilité des conditions de travail peut être rattachée aux caractéristiques du métier choisi, ou encore aux spécificités de l'entreprise de formation (taille, organisation du travail, etc.). Dans certains témoignages, les apprenti.e.s soulignent la question des horaires, des cadences ou du stress généré par celles-ci. Ces éléments révèlent une difficulté commune, celle de jeunes hommes et jeunes femmes sortant de l'école et devant affronter les rudesses du monde du travail. Ainsi, les conditions de travail 
peuvent être analysées sous l'angle de la transition. Chez Andrew, un ferblantier de 17 ans, c'est la pénibilité du métier qui pose problème et les conséquences en termes de souffrance sont importantes:

Travailler en tant que ferbl... dans la ferblanterie: ok, mais le fait d'être dehors, ça c'est un désavantage, parce qu'il fait monstre froid. Pendant l'été, ok, ça c'est un avantage, parce qu'il fait chaud, on est dehors, on aimerait bien être à notre place. Mais... l'hiver...il fait trop froid dehors.

[...] Finir à six heures et demie, bosser le samedi: non!

[...] Je vous demande: quelle personne, dans cette terre, a envie de se lever le matin et pis d'aller travailler quand il fait moins cinq le matin?

Ici, il y a un réel intérêt pour la profession, mais les horaires et les conditions d'exercice du métier (extérieur, climat) posent problème.

Des conditions de travail trop pénibles peuvent être dénoncées. C'est ce que fait Frédéric, un cuisinier de 18 ans, qui, avant de commencer son apprentissage, a fait une année dans le même restaurant mais comme plongeur:

Quand j'ai eu commencé l'apprentissage, c'était la même entreprise, ben ils me prenaient toujours plus ou moins comme le plongeur sauf qu'ils me faisaient travailler encore plus pendant l'apprentissage que quand j'étais plongeur. [...] Ils m'donnaient pas mes deux jours de congé par semaine, [...] pis ils me faisaient travailler souvent neuf jours de suite. [...] C'était neuf jours de suite, un jour de congé, six jours, un jour de congé, pis on refait neuf jours, quoi. [...] J'faisais les deux choses en même temps, en fait, la plonge plus la cuisine.

[...] Ben j'lui [à son formateur] ai dit [...] que j'pouvais pas travailler dans les conditions qu' ils me faisaient travailler [...] Moi j'faisais plus ou moins 60 heures par semaine, eux [les collègues] ils en faisaient que $42 \ldots$

Le jeune homme dénonce une situation incorrecte, revendique ses droits. Se faisant, il se positionne en tant que travailleur à part entière, qui refuse de travailler dans de telles conditions. Comme le ferblantier cité précédemment, il se plaint des horaires, de la pénibilité (des rythmes de travail quasi continus). Ce témoignage renvoie aux constatations de Moreau : «dans les récriminations que ces derniers [les garçons] formulent 
à l'égard de leur maître d'apprentissage, domine le lien salarial. D'entrée de jeu, les garçons se positionnent dans une situation de travail dont ils jugent les conditions. Ce sont alors les horaires, le trop d'heures qu'ils dénoncent [...] Les garçons vilipendent le trop d'heures pour le salaire de l'apprenti, les horaires par rapport au salaire, et le trop petit salaire de l'apprenti qui fait les mêmes heures qu'un ouvrier et qui gagne trois fois moins cher» (G. Moreau, 2000 : 79).

On peut situer les différentes situations relevant des conditions de travail sur un continuum. En effet, la pénibilité peut être liée aux conditions «habituelles» d'exercice de la profession (horaires, travail en extérieur), mais aussi à des situations extrêmes. C'est ce que décrit Alexandra, une cuisinière de 22 ans:

C'était assez lugubre. Y'avait des cafards et tout... ohohoh petite bébête! Moi qui adore ça, j'étais très heureuse, là-bas! J'ouvrais mon vestiaire, ça tombait de tous les côtés, hm!

[...] Même eux, ils étaient, ils se tapaient dessus entre eux, tellement ils étaient sous une pression nerveuse. Entre serveurs et cuisiniers ils se tapaient dessus, c'était tout qui volait dans la cuisine, c'était, euh... c'était vraiment tendu, hein! Comme moi, j'étais là, j'assistais à tout ça: «Ah! il se passe quoi? »

[...] Là-bas, c'était une vraie prison.

Dans ses propos, la pénibilité est liée non seulement à l'insalubrité, qui provoque une souffrance éthique chez la jeune femme (voir Chapitre 6), mais encore aux rapports de travail marqués par la violence. La description de son environnement de travail comme d'un milieu carcéral est particulièrement révélatrice de l'intense malaise ressenti. Des conditions probablement exacerbées par le caractère inhabituel de la présence d'une femme dans les cuisines. Ainsi, les comportements décrits par la jeune femme renvoient peut-être également à des attitudes virilistes adoptées par ses collègues hommes pour «résister» à l'entrée des femmes dans leur univers professionnel. Outre, la violence physique, la jeune femme décrit d'autres comportements, tels que des attaques sexistes ou racistes, typiques des attitudes virilistes (Chamberland et Paquin, 2008; Eckert et Sulzer, 2007; Molinier, 2000).

Si la question des conditions de travail touche les filles comme les garçons, les premières font en outre l'expérience du sexisme, de discriminations et d'inégalités spécifiques. Ainsi, Laetitia, une employée 
de commerce de 16 ans, dénonce ses conditions de travail (absence de contrat, stress), et en particulier l'inégalité salariale dont elle est victime:

Déjà mon contrat d'apprentissage, ils mel'ont pas envoyé dans les temps, ils s'étaient pas préoccupés, c'était un peu bizarre quoi je ne sais pas.

$[\ldots]$

Mais on me donnait trop, ça me fatiguait trop! [...] Ils me chargeaient trop c'était abusé!

$[\ldots]$

Oui et pisj'ai aussi eu l'impression que c'était parce que j'étais une fille qu' ils étaient comme ça avec moi. J'ai vu dans le contrat d'apprentissage, le jour avant que je sois renvoyée, j’avais demandé de regarder mon contrat d'apprentissage. [...] Pis ils me l'ont montré, tout ça, pis j'ai pu regarder aussi le contrat d'apprentissage de l'autre apprenti, quand il a commencé.

$[\ldots]$

Pis quand il a commencé, il gagnait déjà 700 francs au lieu de 600 [...] Voilà. 700 ou 800 , je crois. [...] Mais là c'est clair ça m’a refroidi, j'ai dit ok, après j'ai demandé, j'ai dit: « Ouais c'est salaud!» J'ai rigolé en disant: «Ouais c'est salaud toi tu as eu plus, 100 francs de plus!»

$[\ldots]$

J'sais pas, j'sais pas du tout. J'sais pas si c'est parce que je suis une fille ou parce que je me suis trop laissé avoir en fait.

Contrairement aux garçons cités précédemment, la jeune femme a plus de difficultés à s'inscrire dans un rapport salarial pour dénoncer ses conditions de travail. Elle est d'abord hésitante («c'était bizarre»), car elle ne sait pas si ce qu'elle souhaite dénoncer repose ou non sur des bases légales. La suite de son propos est beaucoup plus affirmée, en particulier sur la question de la discrimination salariale ${ }^{78}$. Elle dénonce une vraie

78 Mis à part les secteurs professionnels soumis à une convention collective, qui a force de loi et dans laquelle figure la rémunération des apprenti.e.s, il n'existe aucune possibilité de l'imposer légalement. Les associations professionnelles et les cantons font des recommandations en fonction de l'année d'apprentissage (voire du semestre). L'employeur.e garde la liberté de suivre 
injustice, même si elle n'est pas encore sûre de son origine: son statut de femme ou son caractère trop arrangeant. Ce doute souligne qu'elle ne se sent pas «à sa place» dans le monde du travail, qu'elle ose moins que les jeunes hommes évoqués précédemment revendiquer des droits.

\subsubsection{La santé au travail}

La question de la santé au travail sera essentiellement examinée à travers celle de la souffrance au travail (voir Chapitre 6). Seront ainsi analysées les situations où l'expérience en formation professionnelle a un impact à moyen ou long terme sur la santé, par exemple en termes de fatigue et d'épuisement. La question de la santé au travail apparait également comme raison de l'arrêt. Dans cette section, seules les situations où la formation professionnelle met directement en périlla santé del'apprenti.e seront examinées.

Jacques, un menuisier de 18 ans, a eu deux accidents en quelque mois en travaillant sur des machines. Suite à cela, il a développé une phobie des machines et n'a plus pu continuer sa formation. Dans ce cas, on peut parler de problèmes de santé psychologique ou mentale:

Parce que j'ai eu deux accidents avec les machines. Je me suis entaillé le pouce, et pis je me suis entaillé le doigt avec une autre scie encore, pis j'me suis touché le tendon [...] A partir de ça [de l'accident], ben, j'ai commencé à plus supporter la vue des machines. Chaque fois que j'avais un truc à faire sur les machines, je demandais à quelqu'un de le faire et pis voilà.

[...] En gros je... je déteste pas ce métier, c'est juste à cause de la phobie.

Des problèmes de santé au travail sont également apparus chez Anne-Cécile, une paysagiste de 17 ans, dont les conditions de travail étaient physiquement difficiles:

En fait, je m'y attendais, mais je pensais que moi, je pouvais tenir le choc, en fait, et quand je me suis rendue compte que physiquement et mentalement ça suivait plus, ben, à partir de là, il y a plus rien qui a été, pis j'ai dit que j'arrêtais.

[...] C'est lourd, quand on fait des mastiques en pierre, moi je portais les pierres avec la brouette, pis je faisais ça toute la

ou non ces recommandations (http://www.vd.ch/fileadmin/user_upload/ themes/formation/postobligatoire/fichiers_pdf/Normes_indicatives.pdf). 
journée. C'est le soir surtout, tu sens ton dos en arrivant, il fait mal et le lendemain il faut recommencer...

D’autres problèmes de santé au travail, tels que les allergies, sont liés à des environnements de travail spécifiques, la coiffure, les métiers des soins et certains métiers du bâtiment et de l'industrie étant fréquemment touchés. C'est le cas d'Agnès, une peintre en carrosserie de 18 ans:

C’est pas vraiment un métier pour moi, parce que déjà la peinture... je fais des allergies au thinner [décapant] et tout... Là j'avais des monstres plaques sur les mains, faut avoir les ongles bien courts, faut pas de bagues, faut pas de ça, faut pas ci [...] donc je pense que travailler sur des voitures ça le fait pas quoi.

[...] Ben en fait aux cours, on est 5 ou 6 à avoir arrêté [...] Pourquoi ? Parce que ça nous plaisait pas, pour des raisons médicales la plupart, moi j'ai de l'asthme aussi, l'asthme, les peintures, ça allait pas du tout quoi. En plus j'étais une fille donc ça n'allait pas. Je faisais crise d'asthme sur crise d'asthme et tout à cause des produits, de la peinture, je faisais des allergies...

Outre des problèmes de santé divers (asthme, allergies, problèmes de genou), le statut de pionnière de cette apprentie rend encore plus difficile son insertion dans la profession. Il est intéressant de noter qu'elle en fait état comme si cela constituait un élément du même ordre ( En plus j'étais une fille...»). Et en effet, dans son cas, la santé et les mises à l'épreuve liées à son statut de pionnière sont étroitement liées (ce que l'on verra plus en détail dans la section suivante).

\subsubsection{L'insertion pionnière}

Globalement, c'est dans la catégorie de raisons liées au monde du travail que se retrouvent majoritairement les témoignages recueillis auprès des personnes pionnières. Sur 9 personnes en situation pionnière, 7 évoquent les conditions de travail comme raison intervenant dans leur décision. Pour les 2 pionniers, il s'agit même du facteur principal.

L'insertion particulière des pionnières et pionniers explique l'importance de cette raison dans les motifs d'arrêt. Pour les garçons, la pénibilité vient de l'absence de perspectives offertes par des métiers traditionnellement féminins, du caractère répétitif des tâches et de l'absence de reconnaissance sociale de ces professions. Sébastien, un assistant en pharmacie de 16 ans, illustre bien ce ras-le-bol: 
En fait, ce que j'ai fait pendant le stage, je l'ai fait pendant 3 mois. Donc...

[...] A la fin. Ça commençait à devenir un petit peu lassant

[...] ranger les médicaments [...] Et puis la poussière aussi. Ça on me disait que j'étais assez fort. Ouais, je devais nettoyer les étagères.

Les éléments mis en évidence (tâches répétitives, activités jugées dégradantes) renvoient probablement à la découverte de ce qu'implique pour eux une insertion atypique, à savoir une mobilité de genre descendante, un déclassement. Dans des professions traditionnellement féminines, peu ou mal reconnues, ils découvrent le caractère répétitif de l'activité, l'absence de perspectives, les possibilités limitées d'avancement, autant d'éléments qui conduisent fréquemment les pionniers à renoncer (Croisier, 2002; Thiébaud, 2004).

Du côté des filles, les discriminations salariales (voir précédemment), la pénibilité physique, la santé ou encore les violences au travail semblent amplifiées par la situation pionnière, le milieu professionnel devenant particulièrement rigide face à la présence de femmes dans des professions masculines (Eckert, 2007; Guichard-Claudic et al., 2008; Molinier, 2000). Si la pénibilité physique est fréquemment évoquée, elle se double parfois du mauvais accueil fait à la pionnière, comme Alexandra, une cuisinière de 22 ans, en a fait l'expérience:

C’est déjà très dur physiquement comme métier, si derrière moralement c'est lourd... On [ses collègues] est toujours en train de rigoler [...] On les entend rire du fin fond de la cuisine et puis, c'est vachement agréable de travailler comme ça.

Dans ce cas, plusieurs éléments s'ajoutent à la pénibilité physique et finissent par avoir raison d'elle. Outre la dureté du métier, il y a également la violence qui règne dans les cuisines, ainsi que celle qui lui est directement adressée :

Il y a eu des insultes, des menaces... des bousculades [...] Une menace c'est: «Je vais t'en coller une, tu vas t'en prendre une, tu vas être contente!» C'est me prendre, me bousculer puis: «Je vais t'éclater.» C'était ça une menace! [...] Il [le chef de cuisine] criait tout le temps, il savait pas parler sans crier. C'était des insultes, c'était: «Dégage espèce de connasse!»

[...] C'étaient des hommes qui étaient très machos, qui étaient très racistes, qui étaient très...: «La femme n’a aucune place 
dans une cuisine et puis elle ferait mieux de rester à la maison, elle ferait le repassage, ce serait bien plus intéressant!» Tout le temps des trucs comme ça que je prenais dans les dents.

Les conditions de travail sont ici particulièrement pénibles, car les hommes avec lesquels elle travaille refusent sa présence. Elle apparaît comme une intruse, qu'il faut faire partir à tout prix. Pour se faire, le sexisme prend des formes variées, allant des remarques lui signifiant son extranéité à la violence physique.

Le fait d'être pionnière peut parfois exacerber les difficultés, y compris en matière de santé. Agnès, la peintre en carrosserie de 18 ans déjà évoquée à plusieurs reprises, subit des mises à l'épreuve qui aggravent ses problèmes de santé. En effet, blessée au genou, ses collègues l'obligeront à se baisser ce qui a mplifiera son problème (voir les analyses proposées aux Chapitres 6 et 8).

De manière générale, les extraits regroupés dans la catégorie «contraintes du monde du travail» illustrent bien les spécificités du système dual. Se former en alternance signifie se plonger directement dans le monde du travail (Kaiser et al., 2007): «la confrontation au statut de salarié, à son rythme et ses conditions est une épreuve pour les apprentis. Ils en découvrent la réalité dans leur corps, dans leur vie quotidienne» (G. Moreau, 2003 : 209).

Les contraintes du monde du travail sont fréquemment associées à d'autres raisons de l'arrêt, en particulier les relations au travail et l'apprentissage du métier. Cela renvoie à des éléments contextuels ou organisationnels, en d'autres termes à ce qui se passe, au quotidien, sur le lieu de formation. Il est donc tout à fait concevable que dans la même entreprise l'apprenti.e rencontre des problèmes au niveau relationnel, ne puisse pas apprendre son métier et soit en même temps pris.e dans des problèmes structurels lié au monde du travail. On peut par exemple supposer que des questions relationnelles participent de la pénibilité au travail ou encore que des conditions de travail difficiles entachent la qualité de la formation dispensée.

N'apparaissant que rarement comme raison principale d'un arrêt, les conditions de travail semblent fonctionner en tant que facteur aggravant, c'est-à-dire conjointement à d'autres difficultés. Cela s'explique par la spécificité de la population, qui n'a aucune expérience professionnelle. En effet, pour ces jeunes, il est difficile de savoir ce qui est acceptable ou non en termes de conditions de travail, par contre elles et ils sont tout à fait en mesure de déterminer si une relation est satisfaisante ou non, ou encore si la formation dispensée atteint ses objectifs. 


\subsection{Des problèmes de transition}

Dans un tiers des situations $(\mathrm{N}=16)$, l'arrêt est expliqué par des difficultés liées à la transition entre l'école obligatoire et la formation professionnelle. Ces arrêts se divisent en deux catégories: la première renvoie à des questions de choix du métier, la deuxième porte davantage sur des difficultés de passage entre le monde scolaire et le monde professionnel et ne concerne qu'une minorité d'ex-apprenti.e.s. C'est aussi la raison qui est la moins associée à d'autres.

\subsubsection{Le choix du métier}

Dans un certain nombre de témoignages $(\mathrm{N}=11)$, l'arrêt de formation est expliqué par un mauvais choix de métier. Trois cas de figure peuvent expliquer cette situation: le métier choisi ne correspondait pas à l'image que la ou le jeune avait de cette profession avant de commencer la formation ; l'orientation a été faite, faute de places dans le métier souhaité; la personne n'était pas prête à faire un choix professionnel (maturité vocationnelle). Les témoignages des garçons se retrouvent plus nombreux dans ces questions d'orientation professionnelle (9 sur 11).

Dans le premier cas de figure, certains jeunes réalisent, lors de l'entrée en apprentissage, que le choix professionnel fait en fin de scolarité ou lors de leur(s) année(s) de transition ne correspond pas à ce qu'elles ou ils attendaient. Aude, une employée de commerce dans une agence de voyage de 17 ans, l'exprime clairement:

C'était bien! Enfin, c'était une bonne entreprise, bonne ambiance, mais c'est pas le métier qui me correspondait, d'être assise: non.

[...] C'est un peu toujours la même chose, c'est des papiers, des papiers.

[...] C'était pas comme je m'étais imaginée, parce que quand j'étais en stage, justement, c'était en plein été, pis y faisaient les voyages pour les gens, pis moi j’ai vu que ça.

[...] Ça me plaisait pas, quoi, je me voyais pas faire ça pendant encore 3 ans.

Dans ces situations, on peut parler d'une certaine désillusion due à l'écart entre attentes et réalité (Fournier, 2002). On constate qu'elles sont différentes des situations montrées jusqu'ici: l'arrêt est dû à des 
questions d'intérêt, indépendamment de la situation concrète au sein de l'entreprise.

D'autres jeunes ont été plus ou moins contraint.e.s de choisir un métier, dans des secteurs où des places étaient disponibles. Ne pouvant pas entreprendre le métier ou la formation désirée, il leur est plus difficile de se maintenir en formation (Kaiser et al., 2007). Par exemple, Sébastien, 16 ans, a choisi un apprentissage d'assistant en pharmacie, faute de pouvoir poursuivre dans la voie baccalauréat:

J'avais pas les points pour aller au gymnase, de toute façon...

[...] Je pouvais pas faire autrement.

[...] Ben moi, ça me dérangeait pas plus que ça, comme j'avais de toute façon pas les points, je me suis dit ben... «Faut faire quelque chose!»

[...] Ça arrivait à la fin [de l'année scolaire], ben, je me suis dit: «Ben, je fais ça, pis, euh, si c'est pas ça, c'est pas ça! Au moins j'aurai... si c'est ça, j'aurai trouvé un truc.»

La pression de devoir trouver pousse parfois les jeunes à accepter des places sans pour autant connaître le métier en question. Massimo, un carreleur de 17 ans, semble avoir vécu cette situation:

Ouais, pendant le stage, ça m'a plu, et voilà,j'avais rien comme travail, enfin, pour l'année d'après, donc j'me suis dit : «Voilà, je signe.»

[...] J’avais rien trouvé, c'est pour ça qu'j’ai signé.

Ces situations illustrent un système de formation professionnelle duale basé sur un jeu d'équilibre entre contraintes contextuelles (le nombre et le type de places d'apprentissage) et aspirations des jeunes (métier idéal). Lorsque cet équilibre est compromis et que les jeunes ne trouvent pas de formation correspondant à leurs aspirations, il leur faut recourir à des choix par défaut (Heinz, Kelle, Witzel et Zinn, 1998; Rastoldo, 2006). Or, ces circonstances influencent négativement les chances de se maintenir en formation (Vonthron, Lagabrielle et Pouchard, 2007), les jeunes étant moins impliqué.e.s dans leur projet professionnel et réussissant plus difficilement à supporter d'autres problématiques (relations, conditions de formation et de travail).

Par ailleurs, certaines personnes ne semblent pas encore prêtes à faire un choix professionnel. Il s'agit surtout des plus jeunes, qui 
manquent de maturité vocationnelle (Forner et Dosnon, 1991) et pour qui l'obligation de faire un choix est intervenue trop tôt. Dans ces situations, certain.e.s choisissent un peu au hasard ou suivent les choix de leur entourage (camarades de classe, ami.e.s). Robert, un charpentier de 16 ans, se laisse par exemple influencer par un ami :

Moi, au début, je voulais faire dessinateur en génie civil, ça a un peu rien à voir... Pis, y a un copain, il a fait charpentier, il a fait un stage, et je me suis dit: «Ouais, je fais, j'essaie un stage, et si ça me plaît, ben, je fais ça, et après la matu [maturité = baccalauréat], et après je fais dessinateur !»

Dans un contexte tendu, l'orientation met en évidence divers enjeux: trouver une formation pour avoir un métier, mais aussi trouver une place d'apprentissage dans un marché difficile. La pression est ainsi ressentie non seulement par les jeunes, mais également par leur entourage. Le cas de Virginie, une employée de commerce de 17 ans, est particulièrement illustratif. Elle a en effet laissé sa mère décider:

C'est ma mère, surtout, qu'a choisi pour moi...

[...] Pour moi, à 16 ans, c'est pas l'âge où on peut savoir ce qu'on veut dans la vie. Même 17 ans, pour moi c'est trop tôt. Je pense que quand on sait ce qu'on veut dans la vie, c'est à partir de 19-20 ans.

[...] Voilà, c'est un peu ça, je savais pas dans quoi je m'embarquais, en fait, tout simplement.

Ces deux derniers exemples mettent en exergue le fait que le système de formation professionnelle duale oblige les jeunes à faire des choix extrêmement jeunes, ce qui ne leur permet pas de bénéficier de la phase de moratoire, contrairement aux jeunes optant pour une formation plus académique (gymnase, lycée). L'indécision vocationnelle, caractérisant les adolescent·e.s et les adultes émergeant.e.s et conduisant à une résiliation du contrat de formation professionnelle, participe pleinement des difficultés de transition entre école et marché du travail. Elle oblige alors à se réorienter, à peaufiner ses choix et à réentreprendre des démarches de recherche de place d'apprentissage, ce qui n'est pas toujours facile (Palmonari et Sarchielli, 1993).

Relire les raisons liées àl'orientation dans une perspective de genre est particulièrement intéressant. En effet, le choix par défaut des filles peut dans certains cas être analysé en termes de choix «raisonnables» 
(Couppié et Epiphane, 2004; Duru-Bellat, 1990), les filles optant pour ou étant orientées dans des filières traditionnelles pour leur sexe, indépendamment de leurs envies ou profils, ce qu'exprime Aude, l'employée de commerce de 17 ans citée plus haut:

C'était une bonne entreprise, bonne ambiance, mais c'est pas le métier qui me correspondait. D’être assise, non! De pas bouger, de rester assise.

Virginie, l'employée de commerce de 17 ans citée plus haut et dont le choix a été effectué par sa mère se retrouve dans une situation similaire:

J'étais pas faite pour le bureau [...] J'suis vraiment pas à l'aise dans un bureau; Enfin, j'ai besoin de bouger.

Ces deux exemples mettent en scène des jeunes femmes ne se plaisant pas dans des professions pourtant conformes à leur sexe. En outre, employée de commerce est une profession socialement valorisée, puisqu'elle requiert des exigences scolaires relativement élevées. C'est donc un «bon» choix, tant en termes d'adéquation aux normes de sexe qu'en termes de prestige social. Pourtant, le caractère sédentaire de l'activité déplaît à ces deux jeunes femmes, qui se heurtent au fait que la mobilité ou l'activité en extérieur sont des caractéristiques attachées aux professions traditionnellement masculines. Ce sont d'ailleurs justement ces traits qui y attirent certaines femmes (Marry, 2007). Les envies et aspirations des deux jeunes femmes sont donc en porte-à-faux avec les normes de sexe attachées à ce métier «féminin » choisi par défaut.

Quant au choix par défaut des garçons, il se heurte parfois à la réalité du statut d'apprenti, et en particulier aux tâches auxquelles il renvoie. Massimo, carreleur de 17 ans, est confronté à cette situation de déclassement, déjà évoquée précédemment:

J'avais rien comme travail, enfin pour l'année d'après, donc j'me suis dit voilà, je signe [...] Ouais, mais j'avais rien trouvé, c'est pour ça que j’ai signé.

[...] Faire de la manutention tous les jours! Au bout d'un moment, ça... [...] Enfin, mettre des carreaux pendant trois ans...

Au-delà d'un simple choix par défaut dicté par un manque de places d'apprentissage, ce jeune homme dénonce à la fois le caractère répétitif de la tâche («mettre des carreaux pendant trois ans») et le caractère socia- 
lement déclassé de l'activité ou des tâches qui lui sont assignées comme apprenti («faire de la manutention»). La situation de Sébastien, l’assistant en pharmacie de 16 ans évoqué précédemment, est quasi identique. Le jeune homme relevait le caractère répétitif ( ranger les médicaments») et déclassé de l'activité («nettoyer les étagères»). C'est cet ensemble de facteurs qui va les conduire tous deux à mettre un terme à leur formation. Outre l'importance pour les garçons d'être reconnus dans un statut de quasi salariés, ces deux exemples montrent qu'il leur est pénible, au point de devenir insupportable, d'être assigné à des tâches subalternes et répétitives (Devreux, 1992). Cela d'autant plus que ces activités les renvoient à la position des femmes dans la division sexuelle du travail, position qui peut être vécue comme une mobilité de genre descendante.

Le sentiment de déclassement peut aussi être lié à l'absence de perspectives professionnelles. C'est ce que ressent Daniele, un opticien de 17 ans, qui constate une erreur d'orientation. Il ne voit dans cette profession ni possibilité de carrière, ni conditions salariales intéressantes:

Moi je pensais beaucoup à l'avenir, le métier d'opticien maintenant, tu fais 4 ans, t'es très mal payé. C’est très très mal payé avec les études que tu fais, parce que c'est pas facile, hein, c'est pas donné à tout le monde, de bosser ses cours... C'est 4 ans, t'as des horaires de travail, c'est pas facile, moi je trouvais que 4 ans, être payé 4200 balles, c'est pas vraiment bien. En plus, si tu fais que les 4 ans, c'est pas vraiment utile, c'est mieux de faire encore la matu [maturité], si tu fais pas la matu, c'est pas que ça sert à rien, mais tu seras simple employé toute ta vie, pis ça j'ai pas envie moi. J'ai envie de m'épanouir au niveau du travail, on dit que l'argent fait pas le bonheur, mais ici en Suisse, si t'as pas ça, tu fais rien!

Cet exemple rappelle que dans le choix d'orientation se joue tout l'avenir professionnel. Le jeune homme anticipe un parcours professionnel qu'il voudrait ascendant. En visant un travail épanouissant et rémunérateur, il anticipe probablement aussi son rôle social d'homme pourvoyeur principal (male breadwinner). Ne serait-ce pas là, la version masculine du choix «raisonnable», une orientation leur permettant de répondre aux attentes sociales qui leur sont adressées : pourvoir aux besoins financiers de leur (future) famille et se surinvestir dans la sphère professionnelle (Eckert et Sulzer, 2007; Vouillot, 1999). 


\subsubsection{Le passage entre l'école et le travail}

La deuxième catégorie de raisons liées aux questions de transition recense les difficultés rencontrées par certain.e.s jeunes face aux changements entre le monde scolaire et le monde professionnel: la modification de l'environnement physique ou humain, les différences de rythmes $(\mathrm{N}=8)$. Elles apparaissent de manière diffuse dans les témoignages de 5 garçons et 3 filles. Une seule personne présente de manière explicite le caractère anxiogène de ce passage. Il s'agit de Laetitia, une employée de commerce de 16 ans:

J'avais envie et pas envie en fait, ça me faisait un peu peur [de commencer après le stage]. Vu qu'après c'était 5 semaines de vacances [...], j'avais un peu les boules, quoi j'étais un peu stressée, j'étais: «Faut pas que je fasse un truc de travers!»

Il n'est guère surprenant qu'une jeune femme se sente autorisée à évoquer son inquiétude face à l'entrée dans le monde du travail. Sa socialisation, de même que les attentes sociales à l'égard des personnes de sa catégorie de sexe, l'autorise à faire état de sa peur (Hurtig, 1998). Pourtant, elle signale en même temps son extranéité face à un monde professionnel, qui ne se présente pas à elle comme une évidence (G. Moreau, 2000). L'univers du travail encore inconnu lui inspire des craintes. Dans l'entretien, elle évoque d'ailleurs la pression subie, les cadences exigées, comme pour confirmer que ses appréhensions face aux attentes du monde professionnel étaient justifiées. Le passage vers ce monde nouveau génère une angoisse, celle de ne pas être à la hauteur des tâches exigées. Evoquée par la jeune femme ci-dessus, elle se devine également chez Liam, un laborantin en chimie de 17 ans, même s'il met surtout en avant la pression générée par ses activités:

C'était un peu monotone. Et stressant, surtout, parce qu'ils me donnaient plein de dossiers à lire, parce qu'il y avait plein de règles d'entreprise, des règles, des... ils me donnaient un tas de dossier à lire, quoi, pis je devais, parce que moi je suis pas très bon en organisation, donc je devais m'organiser pour lire ça et pis en plus je devais m'organiser, si je devais faire les médicaments, si je devais m’organiser pour finir ça à telle heure et puis, ensuite, je peux lire un peu et tout ça donc, en fait, j'ai trouvé ça super stressant.

Les nombreux changements qui interviennent lors du passage entre l'école et le monde du travail provoquent du stress. Il y a de nouvelles 
règles, celles de l'entreprise, qui remplacent celles de l'école, mais il y a aussil'apprentissage de la gestion du travail, de l'organisation des tâches.

La transition entre école et monde du travail met également en exergue des difficultés de passage entre un monde de jeunes (les camarades de classe) et un monde d'adultes (les collègues en entreprise). Certain.e.s apprenti.e.s disent s'ennuyer, ne pas être intéressé.e.s par ces relations avec des adultes, d'autres rappellent l'importance de dissocier sphère professionnelle et sphère privée. Le témoignage de Sylvie, une spécialiste en restauration de 16 ans illustre ces différents aspects :

Quand, peu à peu, on a changé de personnel, donc du personnel plus âgé, qui avait d'autres soucis que ses copains-copines à l'école et ses petites sorties du samedi soir... et ben, en même temps, ce qu'ils se disent, qui causent, on s'en fout, mais voilà, quoi. [...] Moi, j'ai pas à savoir ce qu'ils font dans leur vie privée, moi je voulais, j'étais là pour travailler, pour apprendre, pour faire mon travail.

Les arrêts de formation liés à des problématiques de transition peuvent être considérés à part. Les associations entre cette raison et les autres sont en effet moins fortes. Ici c'est moins les liens avec d'autres raisons qui semblent pertinents qu'une relecture des différentes raisons à l'aune de la transition (Lamamra et Duc, 2015). En effet, derrière les différentes raisons de l'arrêt des questions de transition peuvent être mises en évidence. Les relations au travail soulignent le changement d'environnement qui s'est opéré lors du passage en formation professionnelle, les jeunes passant d'une sociabilité adolescente (école secondaire) à une sociabilité adulte. Les difficultés dans l'apprentissage du métier mettent en évidence un changement d'encadrement et de rythme scolaire (alternance entre formation en école par des enseignant.e.s et formation en entreprise par des formateurs ou formatrices, voire par des collègues, rythme hebdomadaire). Enfin, les difficultés du monde du travail mettent en évidence un passage vers d'autres rythmes (horaires, productivité), d'autres contraintes (hiérarchie, organisation et division du travail).

\subsection{Des contingences externes}

Le dernier type de raisons renvoie à des situations dans lesquelles les personnes ont été contraintes à l'arrêt à cause d'éléments sans liens directs avec leur formation. Les événements qui interviennent dans d'autres sphères de la vie des apprenti.e.s et qui ont un impact sur le déroulement de leur formation seront donc qualifiés de contingences 
externes: problèmes familiaux, problèmes médicaux indépendants de l'exercice de l'activité, etc.

La première situation renvoie à une question générale de santé (qui n'a aucun lien avec la formation entreprise), Stéphanie, une employée de commerce de 19 ans, explique :

Mon problème, c'est que je suis épileptique, et je suis obligée de le dire.

[...] Quand ils ont appris, les gens, quej'étais épileptique, c'est: «loin!» Parce qu'en fait, au début on pensait ne pas le dire, mais après on a remarqué qu'avec les papiers de l'assurance, on ne peut pas ne pas le dire.

Cette maladie influence les chances d'insertion de la jeune femme, notamment parce qu'elle la précarise sur le marché des places d'apprentissage. En effet, peu d'aménagements sont mis en place pour les personnes atteintes dans leur santé. Dans ce cas, le fait d'être épileptique peut être considéré comme une raison contribuant à l'arrêt de formation. D'une part, la patronne utilise l'argument de la maladie pour justifier son envie de rompre le contrat; d'autre part, l'apprentie évoque des difficultés d'intégration à l'école professionnelle. Il serait intéressant de réfléchir à cette situation comme à l'une des possibles discriminations à l'embauche, les futur.e.s employeur.e.s ne souhaitant vraisemblablement pas avoir affaire à des personnes ayant des problèmes de santé ou un handicap, puisqu'elles et ils cherchent à minimiser les risques «de problèmes supplémentaires» (Imdorf, 2007b: 4).

Une autre situation extérieure à la formation entreprise concerne Miguel, un opticien de 20 ans. Il décide d'arrêter son apprentissage lorsque sa fiancée tombe enceinte. Il doit en effet subvenir aux besoins de sa nouvelle famille, en sachant qu'il ne pourra pas obtenir de bourse:

Comme j'avais dit à mon chef d'apprentissage, c'est vraiment le côté vie privée, en fait, qui a fait que j’ai arrêté.

[...] Quand on a su que ma fiancée était enceinte, ben on s'est dit que... je pensais peut être plutôt à arrêter.

[...] Et puis c'est clair que là c'était un salaire d'apprenti, en travaillant quand même une partie du temps chez mon frère ou autre, ça me fait quand même un revenu beaucoup plus élevé. 
[...] S'il y aurait... ben rien que le fait que j'aurais pu avoir la bourse ou bien quelque chose qui, qui au niveau financier, ça aurait joué, c'est clair que j'aurais continué sans problème.

Cet exemple illustre l'impact des différentes sphères de vie sur le déroulement de la formation professionnelle. Il met en outre en exergue de manière originale ${ }^{79}$ la question du conflit entre statut d'apprenti et rôle social d'homme pourvoyeur. Le salaire d'apprenti ne permet en effet pas d'entretenir une famille, et dans une telle situation il semble préférable au jeune homme d'abandonner sa formation - et la possibilité à moyen terme d'occuper un emploi qualifié - et d'opter pour un emploi non qualifié, immédiatement rentable.

Même si elles sont peu nombreuses, ces situations indiquent que la sphère professionnelle n'est qu'un aspect parmi d'autres dans la vie d'une personne. Les autres sphères de vie ne sont pas sans influence sur le parcours professionnel et de formation des jeunes. Ce cas permet donc de mettre l'accent sur une cause peu traitée des arrêts prématurés d'apprentissage ou des décrochages scolaires: la parentalité. Aucune donnée à ce propos dans les études quantitatives. Il semble que cette réalité tend à disparaître dans la catégorie "raisons personnelles», qui n'est pas investiguée plus avant (Stalder et Schmid, 2006a). Il serait pourtant intéressant de connaître l'ampleur du phénomène. En effet, cela permettrait d'envisager la parentalité comme cause d'arrêt prématuré d'apprentissage, comme cela a été fait par rapport à l'échec scolaire (Allen, Philliber, Herrling et Kuperminc, 1997; Dolch, Orr et Ezernack, 2001) et d'envisager des pistes pratiques ad hoc.

\subsection{Le genre comme processus transversal}

Bien que toutes les raisons aient été évoquées par les filles comme par les garçons, certaines d'entre elles sont davantage l'apanage de l'un ou l'autre sexe ou encore les réalités qu'elles recouvrent diffèrent. Ces écarts ne sont pas dus à une hypothétique différence ontologique entre les sexes, mais à l'insertion dans des réalités professionnelles marquées par la ségrégation. Ainsi, derrière une apparente uniformité des situations conduisant à des arrêts prématurés de formation, un certain nombre d'éléments différencient les parcours des jeunes en raison de leur sexe.

79 La question du rôle social d'homme pourvoyeur se pose plutôt dans cette population en termes d'anticipation (voir à ce propos le Chapitre 3 sur l'orientation professionnelle). 


\subsubsection{Des raisons différentes selon le sexe?}

Dans les différentes raisons expliquant un arrêt certaines sont plus évoquées par l'un des groupes de sexe, c'est le cas des «relations au travail» et de la «transition».

Les « relations au travail» sont davantage soulignées par les jeunes femmes ( $\mathrm{N}=16 / 27$ situations). Outre la socialisation traditionnelle, qui encourage les filles à développer des compétences relationnelles et en fait une "qualité» attendue sur le marché du travail (Baudelot et Leclerq, 2005; Dafflon-Novelle, 2006; Hurtig, 1998), les rapports de genre sont à l'œuvre dans les relations au travail. En effet, ce qui est mis en avant dans les récriminations des apprenties, c'est la place particulière qu'on leur assigne dans le marché du travail: une position subalterne, non seulement en raison de leur statut d'apprentie, mais encore de leur sexe. Les jeunes femmes subissent les effets des rapports inégalitaires entre les sexes, sont renvoyées à des positions dévalorisées dans le rapport social et à des représentations stéréotypées, et font l'expérience, à leurs dépens, de la division sexuelle du travail. Le fait qu'elles soient très jeunes et pas encore familiarisées avec le monde du travail offre des témoignages plus crus, plus directs, comme on l'a vu dans ce chapitre. En particulier lorsqu'elles essaient de pénétrer dans les «bastions masculins», elles sont confrontées à une forte résistance de la part de leurs collègues. Les hommes expriment leur opposition à l'entrée des femmes de façon particulièrement explicite sous forme de remarques ou d'agressions sexistes, de mise à l'épreuve (bizutages), parfois de violence (Marry, 2007; Molinier, 2000).

La transition est aussi une raison qui se distingue selon le sexe, car elle est plus fréquemment évoquée par les garçons ( $\mathrm{N}=11 / 16$ situations). Leur entrée dans le marché des places d'apprentissage est plus directe que celle des filles, car ils passent moins par des structures de transition (Imdorf, 2004), et ce malgré des qualifications scolaires tendanciellement plus faibles que ces dernières (Meyer, 2004). A compétences égales, les employeurs privilégient les garçons (Couppié et Epiphane, 2004 ; Imdorf, 2004); et «à qualification égale, les hommes sont significativement plus nombreux que les femmes dans les places d'apprentissage des branches professionnelles qui jouissent d'une image plus prestigieuse» (Imdorf, 2004 : 110). Cette entrée plus rapide, à un très jeune âge, peut avoir des incidences sur le déroulement de la transition, les garçons bénéficiant moins d'un temps d'essais-erreurs, pourtant indispensable à ce moment de construction identitaire. En outre, comme cela a été relevé à plusieurs reprises, le choix professionnel des garçons est souvent un choix d'anti- 
cipation de leur futur rôle d'homme pourvoyeur. Il s'agit donc pour eux de trouver une profession dans laquelle ils pourront s'investir et gagner suffisamment d'argent pour "tenir leur position" dans les rapports sociaux de sexe. Premièrement, cet ordre social met les jeunes hommes sous pression dans la mesure où tout est fait pour qu'ils évitent d'entrer dans des professions (notamment féminines) qui ne leur permettraient pas de remplir cette fonction (Imdorf, 2007a, 2007b). En second lieu, ils font, au même titre d'ailleurs que les filles, des choix de métier fortement sexués, qui participent à la perpétuation de la ségrégation professionnelle (Couppié et Epiphane, 2004).

\subsubsection{Derrière des raisons semblables, des réalités professionnelles qui diffèrent}

Au-delà des raisons plus fréquemment évoquées par les jeunes de l'un ou l'autre sexe, d'autres différences plus fines sont à relever. Ainsi dans la catégorie "apprentissage du métier», la question des performances concerne surtout les garçons, pendant que les filles sont davantage confrontées à un encadrement défaillant.

La question des performances scolaires peut être lue comme une conséquence de ce qui s'est construit préalablement durant la scolarité primaire et secondaire. En effet, en comparaison avec les filles, les garçons arrêtent plus souvent pour des problèmes de performances. La meilleure réussite scolaire des filles au secondaire (Baudelot et Establet, 1992c; Couppié et Epiphane, 2004; Duru-Bellat, 1990; Mosconi, 1994) semble donc se poursuivre en formation professionnelle. En outre, elles se préoccupent sans doute davantage de combler les attentes de l'école professionnelle (Schmid et Stalder, 2007) que ne le font leurs collègues masculins, tournés vers la reconnaissance de leur statut de salarié (Moreau, 2003). Enfin, malgréleur capital scolaire élevé, les filles tendent à choisir des formations en deçà de leurs compétences (reproduisant le paradoxe maintes fois décrit dans la littérature), Preuve en est, quaprès leur arrêt, elles optent pour une formation plus exigeante (Rastoldo et al., 2009; Schmid, 2010). Au contraire, les garçons font le choix inverse: ils s'engagent plus fréquemment dans des formations professionnelles exigeantes, et connaissent après leur arrêt une mobilité descendante, soit en changeant de niveau de formation, soit en optant pour un autre secteur professionnel moins valorisé (Rastoldo et al., 2009; Sch mid, 2010).

En ce qui concerne les "conditions de formation", elles sont plus fréquemment soulignées par les filles dans leurs récriminations, rappelant qu'elles demeurent au cœur des enjeux de la formation professionnelle 
pour les jeunes femmes. Moins légitimes que les garçons dans ce champ, elles continuent à devoir faire montre de leur droit à être formée (G. Moreau, 2000). Elles sont donc davantage confrontées aux «manques» liés à un encadrement inadéquat, voire à l'absence d'encadrement.

\subsubsection{Des choix par défaut qui cachent des choix "raisonnables"}

Le choix «par défaut» est particulièrement intéressant à analyser dans une perspective de genre. Si dans un premier temps, il semblait recouvrir une réalité liée à une pénurie de places d'apprentissage dans certains secteurs, l'analyse secondaire a souligné qu'il pouvait s'agir de choix «raisonnables». Il y a là un très clair enjeu de genre: «les choix d'orientation sont < instrumentalisés > au service du genre, par la nécessité d'affirmation identitaire en tant que fille ou garçon, femme ou homme» (Vouillot, 2002: 488). Ainsi s'explique en partie la reproduction de choix stéréotypés, les femmes s'orientant dans des filières féminisées et les hommes optant pour les filières masculinisées (voir Chapitre 3). Les situations décrites ici ont permis d'identifier plus précisément l'instrumentalisation décrite par Vouillot (2002). En effet, lorsque le choix se fait par défaut ou est contraint (par le marché des places d'apprentissage ou par la famille), il prend alors la forme d'un choix «raisonnable». A titre d'exemple, la plupart des jeunes femmes sont orientées (par ellesmêmes ou par des tiers) dans des métiers traditionnellement féminins, professions du tertiaire, de service, de soins et la plupart des garçons se retrouvent dans des professions largement masculinisées, métiers de l'industrie et de l'artisanat, métiers du bâtiment.

Dans ce corpus, il faut relever à deux reprises l'invalidation de ces « choix raisonnables " par des jeunes femmes qui ne se reconnaissent pas dans cette assignation. Pour elles, il y a eu conflit entre leur manière d'être, d'agir et l'assignation sociale à un certain type d'activité liée à leur sexe (activité en intérieur, sédentaire, métier de service). Du côté des garçons, les résultats relatifs à leurs choix « raisonnables » sont tout aussi instructifs. Certains d'entre eux quittent une formation qui ne leur offre pas les garanties de pouvoir accéder à ce qui fait le statut masculin, et à quoi une activité professionnelle devrait donner droit: salaire, statut, possibilité de progression, etc. L'orientation choisie ne leur permet pas de s'affirmer en tant qu'hommes, soit parce que la formation retenue ne permet pas d'envisager à terme une progression intéressante en termes de salaire ou d'avancement (métiers atypiques, mais aussi métiers de la vente), soit parce que le statut d'apprenti renvoie à des tâches connotées 
comme féminines. Ces exemples soulignent quel'orientation professionnelle assoit la construction du genre, elle permet aux jeunes femmes et hommes d'affirmer leur degré de conformité avec la norme de féminité et masculinité (Gianettoni et Simon-Vermot, 2010; Vouillot, 2002). Ainsi, il peut y avoir arrêt prématuré de formation si la personne refuse ou ne se reconnait pas dans cette norme, ou si la profession choisie ne permet pas de l'atteindre.

\subsubsection{Des garçons qui se considèrent déjà comme des professionnels}

De manière générale, les garçons se positionnent comme des travailleurs, même s'ils ne sont encore qu'apprentis. Ils se placent dans un rapport salarial, cela y compris lorsqu' ils dénoncent leurs conditions de formation. Cet élément mis en évidence par Moreau (2000) est apparu ici de façon transversale aux différentes raisons invoquées. Ainsi, certains garçons parlant de contrat non respecté ou de statut, dénoncent les conditions de travail et les horaires. De plus, même si cela est relativement rare, ils se plaignent de leur salaire d'apprenti ou de celui de leur future profession. Enfin, la question relationnelle se pose en termes d'ambiance, ce qui renvoie clairement à un collectif de travail, dont ils jugent le climat.

Derrière cette volonté d'être reconnus comme des «presque professionnels» se joue sans doute le refus du statut subalterne d'apprenti et des tâches qui y sont associées. Apparaît ainsi en filigrane le fait que l'assignation à de telles tâches, traditionnellement considérées comme subalternes et féminines, fait partie intégrante del'organisation du travail et de l'apprentissage des rapports sociaux au sein de l'entreprise (voir Chapitre 8). L'enjeu est alors pour ces jeunes hommes de se dégager le plus rapidement possible de ce statut et de ces tâches, et de passer du côté de ceux qui peuvent les déléguer à "plus inférieur ou plus dominé» (Devreux, 1992: 163). Cette «émancipation» ne pourra se faire que lorsqu'ils auront obtenu le statut d'employé ou de travailleur et profiter à leur tour de leur position dans la division du travail, sexuelle notamment.

\subsubsection{Des filles qui expérimentent la ségrégation professionnelle, la division sexuelle du travail et les violences des rapports inégalitaires}

L'accès à la formation et les relations au travail sont au cœur de nombreux témoignages des apprenties. Les jeunes femmes mettent égalementl'accent sur les discriminations dont elles font l'objet dans le monde du travail, 
largement documentées dans la littérature en sociologie du travail (D. Kergoat, 2000; Maruani, 2006). Ainsi, certaines filles interviewées dans cette recherche dénoncent le sexisme dont elles sont victimes (position dominée dans le rapport social de sexe, renvoi à des représentations dévalorisantes et stéréotypées), les discriminations (inégalité salariale, non-accès aux machines, assignation plus fréquente aux tâches d'entretien) et les violences (verbales, physiques) qu'elles subissent. Les effets négatifs pour les femmes de la division sexuelle du travail traversent leur récit, que ce soit lorsqu'elles sont insérées dans des professions dites atypiques, ou dans les professions mixtes où elles sont davantage encore que leurs collègues masculins renvoyées à des tâches d'entretien, de ménage. En outre, les relations au travail, auxquelles elles tiennent tant, sont elles-mêmes contaminées par l'ensemble des discriminations qui leur sont faites. Ces jeunes travailleuses font ainsi, parallèlement à leur formation professionnelle, le douloureux apprentissage de la domination, dans ces multiples composantes. 


\section{Chapitre 6 \\ Le passage en formation \\ professionnelle: une expérience de la souffrance au travail?}

Les travaux ayant traité des arrêts prématurés en formation professionnelle n'ont abordé qu'indirectement la question de la souffrance. Ils rapportent souvent les symptômes observés à la difficulté d'affronter un tel événement, mais généralement les présentent comme étant la conséquence de la résiliation du contrat.

Dans les travaux récents, les jeunes ayant connu un arrêt prématuré sont présenté.e.s au travers d'un certain nombre de symptômes cliniques (Schmid et Stalder, 2007; Stalder et Schmid, 2006a) que l'on retrouve également chez les personnes se trouvant hors du système scolaire (Ferron et al., 1997). En comparaison avec des jeunes n'ayant pas arrêté leur formation, les apprenti.e.s qui ont résilié leur contrat présentent des troubles physiques, psychiques et ont des comportements à risque. Elles et ils ont ainsi davantage de maux de tête ou de ventre, de problèmes de concentration, de troubles du sommeil, de nervosité et de fatigue, présentent une plus forte anxiété, une moindre confiance en soi et des risques accrus de faire une dépression (Ferron et al., 1997; Schmid et Stalder, 2007; Stalder et Schmid, 2006a). De plus, leur consommation de tabac (Stalder et Schmid, 2006a), d'alcool, de psychotropes et de drogues en général est importante (Eckmann-Saillant et al., 1994; Ferron et al., 1997).

On peut faire l'hypothèse que les troubles décrits par la littérature comme conséquence de l'arrêt sont en fait les manifestations d'une souffrance existant en amont de celui-ci, mais difficile à exprimer plus tôt (Ferron et al., 1997). Il semble en effet illusoire que le malaise diagnostiqué après l'arrêt ne soit pas intervenu lors du processus qui y a conduit et qui peut se dérouler sur plusieurs mois (Stalder et Schmid, 2006a). Il s'agit donc d'aborder la souffrance comme faisant partie intégrante du processus qui conduit à interrompre sa formation professionnelle. Par ailleurs, une seconde hypothèse peut être posée: le type de souffrance serait lié aux différentes insertions professionnelles, elles-mêmes fortement sexuées. Il y aurait donc une lecture possible de 
la souffrance sous l'angle du genre. Cela signifie qu'il s'agit non seulement de questionner l'évocation, dans les témoignages, de la souffrance comme manifestation d'une conformité aux normes de genre (des filles qui en parlent, des garçons qui la taisent), mais encore d'examiner les souffrances particulières des différents secteurs d'activité (professions de service, métiers « traditionnellement » masculins) ou enfin d'aborder la situation spécifique des pionnières. Ce sont ces différentes hypothèses qui seront discutées dans le présent chapitre.

\subsection{La souffrance en formation professionnelle}

Pour certain.e.s auteur.e.s, la souffrance fait désormais partie du quotidien du travail et la "normalité» se définit alors comme un compromis acceptable entre souffrance et défenses mises en œuvre pour y faire face (Dejours, 2000b; Molinier et Dejours, 1997). Bien que la pénibilité physique et certains dangers aient été atténués grâce aux nouvelles technologies, la souffrance persiste sous d'autres formes, en lien notamment avec les nouvelles normes et contraintes de production. Cet environnement met les salarié.e.s de plus en plus sous pression, augmentant le stress, les risques d'accident (Paugam, 2003) et l'épuisement professionnel. De plus, dans ce contexte, l'extension de certaines pratiques de management (par exemple l'évaluation individualisée) accroît les risques de dérapage, conduisant au harcèlement moral ou au mobbing (voir Chapitre 5). Si ces pratiques ne sont pas nouvelles, le contexte dans lequel elles s'inscrivent renforce leur impact. Au même titre que les nouvelles normes de travail, elles contribuent à affaiblir les solidarités et les stratégies collectives de défense, favorisant l'isolement des individus qui ont peur et qui souffrent (Dejours, 2000b).

Après avoir mis en évidence les liens étroits que la formation professionnelle entretient avec le marché de l'emploi, sa réactivité aux nouvelles configurations du monde du travail et aux changements du contexte économique (voir Chapitre $1^{\text {er }}$ ), il paraît aller de soi que les apprenti.e.s expérimentent également, à leur niveau, la souffrance au travail.

\subsubsection{Formation professionnelle et psychodynamique du travail}

Analyser la souffrance des jeunes lors de leur rencontre avec le monde du travail renvoie plus généralement à l'étude de la relation tendue qui peut exister entre l'individu (ici, les jeunes en formation professionnelle) 
et son environnement professionnel. Les approches centrées sur cette tension sont nombreuses (approches ergonomiques, théorie du stress et du coping ${ }^{80}$, etc.) (Massoudi, 2009) et insistent sur les processus d'interaction entre individu et environnement, ainsi que sur la signification subjective que prennent les situations professionnelles rencontrées par la personne (Probst, 2004).

La psychodynamique du travail - qui s'est développée dans les années 1990 dansl'aire francophone, tant dans le champ scientifique que dans celui de la médecine du travail (Salman, 2008) - a été privilégiée ici. Cette approche a été retenue en raison de la place centrale qu'elle attribue à la question de la souffrance (Dejours, 1998, 2000b; Molinier, 2002a; Molinier et Dejours, 1997), définie comme la manifestation d'une lutte entre normalité et maladie (Alderson, 2004). Analysant «les processus psychiques mobilisés par la rencontre entre l'individu [ici : l'apprenti.e] et les contraintes des situations de travail [ici: la formation professionnelle duale]» (Lamamra et Masdonati, 2008), la psychodynamique du travail permettra de comprendre comment les apprenti.e.s font face aux situations qu'elles et ils vivent.

De plus, du point de vue épistémologique, cette approche affirme le primat du terrain, opte pour une démarche compréhensive et place au cœur de son analyse la subjectivité de l'individu, lui conférant le statut de sujet et ce malgré les contraintes organisationnelles (Alderson, 2004; Carpentier-Roy, 2006). D’une part, ce présupposé correspond parfaitement à la démarche inductive utilisée dans cette recherche, qui consiste à faire émerger des résultats les questions de souffrance. D’autre part, une importance primordiale a été conférée au statut de sujet des apprenti.e.s, dans l'idée que c'est bien à partir de cette position que les jeunes peuvent construire leur identité et, dans le meilleur des cas, agir sur leur environnement professionnel (Vézina, 2007).

Ainsi, même si la psychodynamique du travail est surtout appliquée à l'analyse de l'activité professionnelle, il a paru pertinent de la transposer dans le contexte particulier de la formation professionnelle duale, ne serait-ce que parce que l'apprentissage constitue en soi un travail (Leclerc et Maranda, 2002) et s'exerce dans de véritables organisations productives (Lamamra et Masdonati, 2008).

80 Le coping est une stratégie consciente d'ajustement au stress, permettant de minimiser ses effets et de le supporter. 
Par ailleurs, comme en ergonomie, le travail est considéré en psychodynamique du travail comme allant au-delà du travail prescrit ${ }^{81}$ : «Tout le débat que l'ergonomie ou la psychodynamique du travail entretiennent avec l'organisation du travail porte sur la nature de ce ‘quelque chose en plus ', qu'il faut rajouter pour qu'il y ait production » (Davezies, 1993: 34). L'accent est mis sur l'écart entre travail prescrit et travail réel, écart qui peut être source de santé ou de souffrance mentale, selon la marge de manœuvre que l'organisation du travail laisse à l'individu. S'intéresser à l'activité professionnelle sous cet angle semble donc particulièrement fructueux lorsqu'il s'agit d'analyser la situation de jeunes en train d'apprendre le métier. Ne connaissant pas encore l'entier du travail prescrit, et n'osant pas nécessairement s'éloigner de la prescription, on peut considérer que ces jeunes n'ont quasi aucune marge de manœuvre. Car c'est la pratique professionnelle, l'expérience qui permet de s'éloigner de la prescription. Le risque de souffrance des apprenti.e.s dans l'exercice de leur métier est donc bien réel.

Enfin, la psychodynamique du travail s'est imposée car elle a très vite intégré une perspective de genre à son analyse (Hirata et Kergoat, 1998; Molinier, 2004). Toute analyse du contexte organisationnel implique en effet (ou devrait impliquer) une prise en compte de la division sexuelle du travail et de la souffrance, ainsi que des stratégies développées pour faire face aux contraintes de travail spécifiques à des environnements féminins, masculins ou mixtes. En clair, il s'agit d'intégrer dans l'analyse de l'organisation du travail et de l'expérience du travail l'inégalité des positions relatives qu'occupent les femmes et les hommes dans les rapports sociaux de sexe. Les contraintes organisationnelles et le vécu des personnes ne sont pas identiques selon qu'elles travaillent dans le secteur des services, des soins ou dans l'industrie (Molinier, 2000). Les attentes dans des domaines d'activitélargement sexués sont étroitement tributaires des stéréotypes plaqués sur les femmes et les hommes, et de ceux associés à des professions définies comme féminines ou masculines, hiérarchisées les unes par rapport aux autres.

Pour ces différentes raisons, la psychodynamique du travail constitue le cadre de référence avec lequel il est possible d'analyser la souffrance des apprenti.e.s en rupture de formation comme un processus qui prend corps en aval de la rupture. Ce même cadre sera aussi utilisé dans le chapitre suivant, consacré aux stratégies de défense mises en

81 Au sens de l'application des consignes, de la «simple» exécution de tâches définies préalablement par différents acteurs : responsables de production, médecins du travail, etc. (voir à ce propos Davezies, 1993). 
œuvre pour affronter les situations de souffrance. Car la psychodynamique du travail permet de dépasser une compréhension des réponses à la souffrance en termes de ressources psychiques individuelles (résilience ou coping), pour favoriser une analyse en termes de ressources sociales (y compris dans leur dimension psychique) produites dans un contexte de travail, à l'appui de stratégies tant individuelles que collectives.

Malgré sa pertinence, la transposition de cette approche comporte certaines limites liées à la spécificité de cette recherche. En premier lieu, la psychodynamique du travail propose une approche clinique, qui s'intéresse - notamment par l'observation - à l'organisation du travail. Ce n'était pasl'objet ici, les entretiens ont été conduits dans le but d'éclairer le processus menant à l'interruption d'une formation professionnelle duale et non d'analyser les aspects organisationnels. Ainsi, si la question de la souffrance et de la santé a émergé de l'analyse de l'expérience vécue en formation professionnelle, les entretiens n'ont jamais eu de visée clinique. Ils ont en outre été menés après la rupture du contrat d'apprentissage, soit après la fin des rapports de travail, ce qui a rendu impossible toute observation de l'environnement et de l'organisation du travail.

Deuxièmement, la psychodynamique du travail s'intéresse au caractère collectif des stratégies de défense face aux contraintes du travail, notamment en ce qui concerne les stratégies dites de métier ou encore les idéologies défensives (Dejours, 2000b). Or, les apprenti.e.s, par leur statut précaire et provisoire, leur âge, leur insertion récente dans l'emploi, ne constituent que rarement un collectif (voir Chapitre $1^{\mathrm{er}}$ ). Elles et ils sont fréquemment seul.e.s au sein de leur entreprise de formation, n'appartenant pas encore au collectif des " professionnel.le.s" de métier, ce qui peut également expliquer leur souffrance. L'accent sera donc mis sur la dimension individuelle et expérimentale de leurs stratégies, comparées aux stratégies des travailleuses et travailleurs adultes observées par la psychodynamique du travail.

\subsection{Un contact douloureux avec le travail}

Différents aspects participent del'émergence dela souffrance en formation professionnelle. Certaines des raisons évoquées au chapitre précédent laissaient déjà entrevoir des sources de malaise sur le lieu de formation (relations au travail, conditions de formation ou de travail) qui peuvent se transformer en véritable souffrance. De plus, les apprenti.e.s, par leur statut de personnes en formation, n'ayant qu'une marge de manœuvre réduite et ne pouvant pas encore s'éloigner du travail prescrit, il leur est 
difficile de se «réaliser» dans les espaces où elles et ils pourraient faire preuve de création (Molinier, 2000), où elles et ils pourraient sublimer leur souffrance et découvrir le plaisir au travail (Dejours, 1998, 2001 ; Hirata et Kergoat, 1998). Au bas de la hiérarchie, les apprenti.e.s sont particulièrement vulnérables et représentent les premières victimes des dysfonctionnements de l'entreprise, lorsqu'il y en a. Cette impuissance, bien que provisoire, peut également être une source de souffrance au travail.

Les résultats montrent que la peur est un autre élément constitutif de la souffrance. Elle semble même être au cœur de l'expérience de ces adolescent.e.s à peine entré.e.s dans le monde professionnel. Contrairement aux travailleurs et travailleuses adultes, la peur est ici rarement liée aux dangers physiques inhérents au métier: dans la population étudiée, seules trois personnes (un ferblantier, un charpentier et un menuisier) se forment dans des professions que l'on pourrait qualifier de métiers «à risque ${ }^{82}$. Elle tient par contre, dans la plupart des cas, à l'organisation du travail, au statut d'apprenti.e, ou aux spécificités de la formation professionnelle duale. Certaines raisons d'arrêt décrites dans le chapitre précédent se retrouvent ici : la crainte de ne pas être à la hauteur, de ne pas répondre aux attentes de comportement et de productivité d'un nouvel environnement; la peur liée à des tensions relationnelles; la crainte de la pression économique, dans un contexte où les places d'apprentissage sont difficiles à décrocher et où la sécurité d'un emploi à l'issue de la formation n'est pas garantie; enfin, mais dans une moindre mesure, la peur du danger que représente l'activité même, à l'exemple d'un apprenti menuisier qui appréhende en permanence l'accident toujours possible.

Généralement, les collectifs de travail développent des stratégies pour faire face à l'angoisse des risques encourus (Dejours, 2000b). Elles s'élaborent et se transmettent au sein du groupe, elles sont partagées par les membres d'une même «communauté de pratique». Mais les apprenti.e.s, encore au tout début de leur socialisation professionnelle, sont rarement inséré.e.s dans des collectifs, ne connaissent pas les stratégies que ceux-ci mettent en place et se retrouvent donc, de fait, sans défense.

82 On entend ici les professions exposées à des dangers qui peuvent porter atteinte à l'intégrité physique, tels que le bâtiment, les travaux publics, les professions ayant affaire à des produits toxiques, etc. 


\subsubsection{Analyser la souffrance: évocation subjective et jeu de genre}

La souffrance étant une notion complexe et prenant de multiples formes, elle n'est pas facile à identifier. Il a donc été considéré qu'il y avait souffrance chaque fois que les personnes interviewées l'évoquaient. Ainsi, toutes les situations de souffrance auto-reportées ont été prises en compte. En reconnaissant cette seule évocation subjective, aucune tentative de vérification, d'évaluation ou de mesure de la souffrance n'a été envisagée. Précisons toutefois que les données récoltées ne permettent pas de conclure à l'absence de souffrance chez les jeunes qui n'en parlent pas: il peut s'agir d'attitudes de déni, ou d'une parade de genre, certains (les jeunes hommes et parfois les pionnières) ayant des difficultés à exprimer leur souffrance. Par ailleurs, lorsqu'elles en parlent, les personnes en arrêt de formation ne l'abordent pas toujours de manière directe ( $«$ je souffre»), mais y font plutôt allusion en évoquant certains symptômes ( «je ne mangeais plus», «je ne dormais plus»). L'analyse a donc dû composer à partir de ces éléments explicites ou implicites.

Dans un premier temps, la souffrance semble davantage faire partie de l'expérience des jeunes femmes. Elles sont en effet plus nombreuses à dénoncer l'une ou l'autre forme de souffrance que leurs collègues masculins (27 pour 19). Ce rapport de $60 \%$ pour $40 \%$ se retrouve en ce qui concerne la souffrance mentale, et l'une de ses formes la souffrance éthique. En ce qui concerne la souffrance physique, elle apparait àl'identique chez les jeunes des deux sexes (4 et 4). L'analyse de contenu fait apparaître que les jeunes femmes parlent davantage de leur souffrance, qu'elles le font directement et avec force détails sur les causes, les effets, les symptômes ressentis. Les garçons quant à eux se montrent moins prolixes et prennent davantage de détour. Il faut donc se garder de ce faux-semblant, qui crée l'illusion d'une disparité entre les sexes quant à l'expérience de la souffrance en formation professionnelle ${ }^{83}$.

Comme évoqué ci-dessus, lorsque les filles témoignent de leur expérience, elles le font de manière explicite. Elles évoquent ainsi leur peine, leurs larmes, à l'instar d'Oriane, une spécialiste en restauration de 16 ans:

Je m'étais enfermée pendant une heure dans les toilettes, parce que ça allait pas et pis je pleurais toutes les cinq minutes...

83 On retrouve ce mode d'énonciation différencié selon le sexe en lycée professionnel, en ce qui concerne les récits d'actes de transgression (Depoilly, 2014). 
Elles présentent également un certain nombre de manifestations, comme des troubles du sommeil ou des troubles alimentaires, laissant penser à des symptômes dépressifs. Ainsi Alexandra, une cuisinière de 22 ans:

Ça a été dur, quand j'ai arrêté de dormir. J’ai arrêté de dormir et arrêté de m'alimenter. Je ressentais ni le sommeil, ni la faim.

Ou Elisabeth, une spécialiste en restauration de 17 ans, qui utilise même explicitement le terme de dépression:

J'ai été plusieurs fois au docteur, il m’a dit que je faisais une petite dépression.

La souffrance mentale semble dicible pour des jeunes femmes, elle est socialement acceptable. Ayant appris au cours de leur socialisation à faire part de leurs émotions (Dafflon-Novelle, 2006), elles sont autorisées à pleurer, à consulter un médecin, à se considérer comme "faibles", elles peuvent parler précisément de ce qu'elles ressentent, détailler les symptômes de leur malaise. Qu'il s'agisse des larmes, de la dépression ou des symptômes y référant, ces troubles reviennent très fréquemment dans les témoignages des apprenties (30 évocations directes), alors que cela est beaucoup moins souvent le cas dans les propos des jeunes hommes (6 évocations). Pourtant la souffrance fait également partie de l'expérience de ces derniers, mais elle semble ne pas pouvoir se dire aussi clairement, certains garçons utilisant alors des effets de langage ou des images, comme Adnan, un informaticien de 18 ans, qui parle ici de son changement d'attitude au fil de l'expérience qu'il a vécue en formation professionnelle:

Je ne suis plus dans ma peau, quoi!

D'autres rendent la souffrance perceptible en amplifiant leur propos. Présentée sous une forme extrême, celle-ci devient enfin dicible, comme lorsque le registre de la mort est mobilisé par Jacques, un menuisier de 18 ans:

J'commençais à devenir... (rit) mort!

Ou par Alessandro, un peintre en carrosserie de 19 ans :

Moi, je commençais à claquer, moi...

Ces effets de style font probablement partie d'un langage adolescent, mais caractérisent davantage les propos des jeunes hommes. Il faut 
également remarquer la brièveté des phrases prononcées. Des récits détaillés et circonstanciés d'un côté, des évocations brèves et dramatiques de l'autre, tout laisse à penser que le genre se joue aussi au moment où la souffrance doit être dite. Du côté des garçons, il semble difficile de communiquer la souffrance vécue, exprimée en si peu de mots qu'elle paraît avoir péniblement franchi la barrière de la virilité défensive (voir Chapitre 7). En outre, les registres utilisés font penser que pour se dire, la souffrance des garçons doit être légitimée par un caractère dramatique. La stratégie de défense se construit sur une virilité fantasmée, toute puissante, capable de tout endurer. Ainsi, pour ne pas la mettre en péril, il faut que la souffrance soit à la hauteur. La dramatisation de la souffrance sert donc la valorisation de la force (Hirata et Kergoat, 1998). En l'évoquant de cette manière, les apprentis peuvent à la fois signaler leurs limites sans pour autant se trahir en tant qu'hommes, ni trahir le collectif construit autour de cette virilité. Leur crainte - qui semble ici plus forte que la souffrance - étant d'être mis en cause dans leur identité virile, «d'être raillé[s] par les autres, méprisé[s] comme une " chochotte » ou une «femmelette», jusqu'à ce qu' il[s] craque[nt]...» (Molinier, 2000: 30), et d'être exclus du groupe des hommes.

\subsubsection{Une typologie de la souffrance}

La souffrance en formation professionnelle occupe une place centrale dans l'expérience des jeunes et apparaît de façon transversale, quelles que soient les raisons évoquées pour expliquer l'arrêt prématuré de formation. Les propos des jeunes ont permis de distinguer deux types de souffrance. La première est la souffrance mentale, c'est-à-dire la tension entre les contraintes du travail et les stratégies mises en œuvre pour y faire face (Dejours, 1998, 2000b). Cette tension est particulièrement visible dans ce que Dejours appelle la souffrance éthique, soit le résultat de la distorsion entre ses propres valeurs et ce que l'on est amené à faire dans le cadre d'une activité professionnelle (Dejours, 2001). La seconde forme de souffrance analysée est la souffrance physique, qui renvoie aux contraintes du marché du travail (pression au rendement, rythmes, cadences), mais aussi à la spécificité de ces jeunes travailleurs et travailleuses, encore inexpérimenté.e.s, et qui subissent dans leur corps l'apprentissage du travail (G. Moreau, 2003).

Cette typologie issue de la littérature en psychodynamique du travail sera affinée ici par les résultats de l'analyse secondaire sous l'angle du genre. La souffrance éthique sera ainsi renvoyée aux professions de service, pendant quela souffrance physique sera associée aux professions 
traditionnellement masculines. Il sera en outre tenu compte de la population particulière de cette étude, les apprenti.e.s ainsi que de celles qui «transgressent» les frontières de genre en se risquant dans des métiers non-traditionnels pour leur sexe, les pionnières.

\section{La souffrance mentale}

La souffrance mentale est celle des formes de souffrance qui apparaît le plus dans cette population, exprimée par près des $2 / 3$ des apprenti.e.s interrogé.e.s $(\mathrm{N}=28)$. En outre, les filles en parlent plus que les garçons (17 pour 11). La souffrance mentale structure donc l'expérience de la souffrance éprouvée par les apprenti.e.s en rupture de formation.

Dans les entretiens, la souffrance mentale se manifeste fréquemment par un malaise intense lié aux prescriptions ou aux contraintes du travail et suscite chez les personnes un sentiment d'impuissance (Lamamra et Masdonati, 2006). Bien que cette souffrance ait été éprouvée dans le cadre d'une toute première expérience professionnelle, les témoignages des apprenti.e.s (recueillis a posteriori) laissent présager qu'elle les a marqué.e.s durablement et qu'elle peut avoir un impact à moyen terme sur leur manière de se projeter dans l'avenir. Cette suggestion s'appuie sur la violence des situations qu'elles et ils rapportent. Les agressions verbales dont les ex-apprenti.e.s font part témoignent de cette violence, occasionnant une souffrance qui les conduit parfois à ne plus vouloir poursuivre leur formation, à l'instar de Jeremy, un gestionnaire de commerce de détail de 20 ans:

Des fois, il [le formateur en entreprise] arrive, il me gueule dessus comme une merde, comme un chien je crois! Tous les jours, vous voyez un peu comme c'est: «Vas-y, casse-toi là, qu'est-ce qu'on s'en fout de toi!» En tout cas, je me suis vraiment senti comme une merde! Ouais j'avais la rage, vous voyez! J'avais envie de travailler! J'avais une flamme! [I: Et elle est où cette flamme ?] Elle est sous la pluie, je crois qu'elle s'est éteinte.

Certaines expériences peuvent être qualifiées de traumatisantes, tant les effets qu'elles provoquent chez les apprenti.e.s sont violents. Les propos de Leonora, une assistante dentaire de 16 ans, sont particulièrement révélateurs d'un état de désarroi intense:

Le matin, j'allais tous les matins en pleurant. Je prenais même plus le temps de... voilà quoi. C'était le travail, maison et pis dormir. Je parlais à personne, j'avais aussi perdu un peu contact 
avec mes cousines. Pis j'ai pas arrêté de pleurer pendant je sais pas combien de temps. J'avais des infections sous les yeux avec des grosses marques rouges, parce quej'arrêtais pas de pleurer.

L'expression de la souffrance la plus courante est la tristesse, qui peut aller, comme ici, jusqu'à des symptômes dépressifs. En effet, si la souffrance a été identifiée grâce au discours très clair de Leonora sur ses symptômes (larmes, insomnies, silence, isolement), c'est tout le récit de son expérience en formation professionnelle qui permet de comprendre le processus. Des collègues et un patron (le dentiste) hostiles, des agressions à connotation raciste, des difficultés d'intégration au sein de l'équipe, ont raison d'elle, malgré des tentatives de mise en conformité (elle anticipe le travail, en fait davantage).

Les symptômes exprimant la souffrance sont parfois tels que l'on peut véritablement parler de pathologie relevant du stress post-traumatique $^{84}$. Aurélie, une gardienne d'animaux de 17 ans, qui a subi mise à l'écart et épreuves de la part de ses collègues, soit ce que l'on pourrait qualifier de mobbing, l'exprime de façon très claire:

Je dormais plus et puis j'avais une fatigue mentale, donc je dormais, mais je me reposais pas, je faisais des cauchemars toutes les nuits, je mangeais plus, j'avais plus goût à rien, ouais je voyais vraiment la vie en noir et blanc.

Certaines situations extrêmes dévoilent des violences psychiques d'une telle ampleur que la souffrance au travail devient insupportable (OMS, 2002). Elles soulèvent des questions de santé publique fondamentales. Le témoignage de Sarah, une fleuriste de 16 ans, souligne le caractère dramatique des situations de ce type:

Il m’a hurlé dessus comme personne m’a jamais hurlé dessus, moij'avais qu'une seule envie, c'était de me foutre sous le train. J'ai été m'acheter des bouteilles d'alcool, j'ai dépensé je crois 120 balles... tout ça pour me foutre en l'air.

La violence de l'altercation avec l'employeur (ci-dessus) ou avec des collègues met en évidence un aspect peu exploré dans ce travail, soit la confrontation entre des jeunes inexpérimenté.e-s et les stratégies de défense de leurs collègues adultes. La violence verbale, les insultes, les

84 Selon les indications de l'American Psychiatric Association, Diagnostic and statistical manual of mental disorders: DSM-IV. Washington, DC: American Psychiatric Association, 1994. 
agressions en sont l'expression. L'impact de cette confrontation sur la santé mentale des adolescent.e.s peut être majeur. A l'instar de Sarah, certain.e.s peuvent être tenté.e.s d'adopter des conduites à risque pour se protéger. Ici l'alcoolisation pourrait devenir un rempart régulier contre la violence subie au travail.

La souffrance mentale se manifeste au travers de symptômes intervenant à plusieurs niveaux : comportemental, affectif, cognitif ou encore physiologique. Ils montrent l'impact de cette souffrance sur la santé des jeunes, qui développent diverses pathologies: troubles du sommeil («je dormais plus» Aurélie, «je ressentais ni le sommeil, ni la faim» Alexandra), troubles alimentaires («je mangeais rien» Daniele, «je mangeais pas, j'avais pas très faim » Elisabeth) ou violence retournée contre soi («je me mutilais» Tiffany). D’autres symptômes évoquent plus directement un état dépressif («je désespérais» Adnan, «je suis partie en dépression» Tiffany, «je faisais une petite dépression» Elisabeth) et peuvent aller jusqu'à des pensées suicidaires («me foutre sous le train», «me foutre en l'air» Sarah). Il serait intéressant de compléter cette analyse de la souffrance mentale par une analyse des registres linguistiques utilisés. En effet, les termes utilisés par les jeunes pour exprimer leur souffrance peuvent être extrêmement forts.

\section{a) Une composante de la souffrance mentale: la souffrance éthique}

La composante éthique de la souffrance mentale est à prendre au sérieux, «parce qu'elle touche en plein cœur la question de l'identité » (Dejours, 2001 : 2). Dans l'impossibilité de concilier leurs valeurs avec celles, parfois antagonistes, de leur environnement de travail, les jeunes témoignent de la désorientation qu'elles et ils éprouvent lors de la rencontre avec le monde professionnel. On pourrait même parfois parler de désillusion face au monde adulte. En effet, encore adolescent.e.s ou tout juste sorti.e.s de cette phase de leur vie, les apprenti.e.s doivent soudain se confronter à des adultes ayant des valeurs parfois contraires aux leurs. Si cette expérience n'est pas nouvelle, puisqu'elle a déjà été faite durant la socialisation scolaire, le statut d'apprenti.e ne leur permet pas nécessairement de défendre leur point de vue. Dans certains cas extrêmes, les apprenti.e.s doivent même composer avec des demandes illicites ou encore des comportements malhonnêtes de la part de leurs employeur.e.s (marchandise non-déclarée, mauvaise qualité du service, concurrence déloyale, etc.). L'image que ces jeunes se font du monde du travail et du monde des adultes peut s'en trouver ternie. 
Deux types de situation semblent être à l'origine d'une souffrance éthique: les manquements à l'éthique professionnelle et les problèmes relationnels. Concernant l'éthique professionnelle, il est intéressant de noter qu'elle compte pour ces jeunes qui n'ont pourtant pas encore terminé leur formation, voire qui en sont au tout début. Et si les apprenti.e.s ont déjà une image forte de la manière dont elles et ils doivent se comporter dans le monde du travail, on peut comprendre qu'elles et ils soient parfois déçu·e.s de la réalité rencontrée. C'est le cas de Oriane, une spécialiste en restauration de 16 ans :

Pour un hôtel, ben déjà, ils ont pas de restaurant, ce qu'on vend pour le room-service, c'est que des plats surgelés et tout vient de chez [magasin de vente en gros], alors j'étais un peu heu, enfin... très surprise en fait, et je trouvais ça un peu dégueulasse vis-à-vis des clients, j'essayais pas trop de parler, parce que j'ai plutôt honte de travailler là-bas.

Dans le même ordre d'idée, l'éthique professionnelle exige une attitude correcte vis-à-vis de la concurrence, à l'instar de ce qu'en dit Jacques, un menuisier de 18 ans :

Ils avaient une réputation de voleurs de boulot. Ils font encore les prix plus bas que la normale. Quand j'ai entendu ça, je me suis dit qu'est-ce que j'ai foutu là-dedans.

Ces deux citations illustrent l'existence d'un malaise réel chez certain·e.s apprenti.e.s, malaise qui naît de l'écart entre leurs propres valeurs, ou de leurs représentations du «juste» et celles de leurs employeur.e.s. Les jeunes ont alors honte et se distancient de l'entreprise qui les emploie.

La seconde forme de souffrance éthique intervient lors de problèmes relationnels. C'est peut-être dans ce cas de figure que l'on peut véritablement parler de désillusion face au monde adulte. Cette déception est ressentie notamment par Matthieu, un vendeur en pièces détachées de 16 ans:

Ils se faisaient des coups dans le dos et tout ça et moi justement je voulais pas me mêler de ces affaires, je disais par exemple que j'étais contre ça, que je comprenais pas pourquoi les gens ils faisaient ça. Je disais: «Non, moi je comprends pas, moi j'aime pas dire du mal des gens dans le dos!»

Dans ces différentes situations, les jeunes, qui ne se reconnaissent pas dans les pratiques qui les entourent, éprouvent un réel malaise. Que ce 
soit face aux manquements à l'éthique professionnelle ou à des problèmes relationnels, cette souffrance met en lumière le sens particulièrement aigu de la justice qu'ont les jeunes. Elles et ils tolèrent moins ce qui est considéré de l'ordre de "l'injuste» (Angelini et Esman-Tuccella, 2004). Par leur âge et leur entrée récente dans le marché du travail, les apprenti.e.s sont moins pris.es dans des stratégies défensives, elles et ils sont plus aptes à se révolter, à relever l'injustice. Pour autant, elles et ils cherchent à mettre en œuvre des stratégies pour faire face à ces situations, tentant par exemple de prendre de la distance, en ne parlant pas de ce qui se passe, en adoptant une certaine forme d'extériorité, ou encore en refusant de prendre part à ce qui les met mal à l'aise. Faut-il alors comprendre l'arrêt de formation comme une volonté ultime de prendre ses distances? Auquel cas l'arrêt pourrait signifier une mise en adéquation avec ses propres valeurs. Il s'agirait alors d'une solution éthique pour sortir de la distorsion créée par l'environnement de travail, l'arrêt n'est alors pas un échec.

\section{b) La souffrance éthique, une caractéristique des métiers de service?}

Un élément déterminant est à relever ici: la plupart des jeunes faisant état de souffrance éthique sont inséré.e.s dans des professions de service. En effet, sur les 10 personnes recensées, 9 se forment dans des métiers de service (vente, bureau, restauration, coiffure et esthétique). Ceci suggère qu'il existe un lien entre le type d'activité et la souffrance éprouvée. $\mathrm{Si}$, à certains moments comme on l'a vu, les souffrances des filles et respectivement des garçons se distinguent, c'est parce qu'elles et ils n'exercent pas le même métier: «certaines souffrances sont masculines, d'autres souffrances sont féminines. Elles ne sont pas similaires, parce que les situations qui les génèrent ne sont tout simplement pas les mêmes» (Molinier, 2000 : 27). Dans le cas présent, la souffrance générée est identique, car filles et garçons travaillant dans ces métiers de service souffrent du fait que l'environnement de travail ne garantisse pas la qualité de ce service et ne mette pas la clientèle au cœur de l'activité.

Il s'agit donc ici d'un effet de genre et non d'un effet de sexe: filles et garçons intégré.e.s dans les métiers de service se montrent particulièrement sensibles à la façon dont la clientèle est traitée et soignent leurs propres relations avec celle-ci, commeStella, une esthéticienne de 17 ans:

J'estimais que je faisais mon travail, que par rapport aux clientes j'étais restée super professionnelle [...] C'était mon devoir envers les clientes. 
Compte tenu de la concentration des femmes dans les professions de service et de soins aux personnes, l'on peut supposer que dans l'ensemble de la population des apprenti.e.s, les filles sont plus nombreuses que les garçons à se confronter à la question du rapport à la clientèle. En outre, dans ces métiers de service et de soins, les compétences professionnelles recouvrent fréquemment les «fameuses qualités » féminines, d'attention à autrui, de patience, d'écoute (Marry, 2006). Pour les filles, la souffrance éthique semble ainsi doublée d'une composante supplémentaire: à la distorsion qu'elles rencontrent entre les valeurs qu'elles avaient associées au métier et les conditions dans lesquelles elles doivent le pratiquer, s'ajoute le fait que les valeurs projetées sur la profession sont censées constituer une part centrale de leur féminité. Ne pas disposer des conditions nécessaires à l'exercice correct de leur activité revient ici à ne pas pouvoir faire preuve de féminité.

Les garçons insérés dans ces professions de service accordent une attention particulière à l'image de l'entreprise. Ils s'insurgent contre les pratiques déloyales ou frauduleuses de certaines entreprises, montrant ainsi leur attachement à la qualité du service. Ces pratiques sont par exemple dénoncées par Jeremy, un gestionnaire de commerce de détail de 20 ans :

Il [le patron] m’a envoyé en France chercher de la marchandise! Sans déclarer à la douane, parce que c'est des choses qu'on peut pas vendre ici. [...] Moi je fais ce qu'il me demande hein, je vais pas dire non! [...] Il me disait: « T’as trois heures pour aller vers la France, prendre les trucs...» [...] En plus, que je passe la frontière comme ça en cachette. Une fois, je me suis fait choper, voilà, et j'ai dû partir.

Dans cet extrait, le jeune homme signale qu' il ne supporte pas les contournements éthiques de son employeur. Il illustre ainsi son sens aigu de la justice, et c'est dans ce cadre qu'il faut lire la dénonciation qu'il fait en parallèle de ses conditions de travail. En plus des pratiques illégales, Jeremy dénonce effectivement une situation d'exploitation, d'abus.

L'accent mis par les garçons sur les conduites de l'entreprise s'explique peut-être par la manière dont ils endossent leur statut d'apprenti.e. Comme cela a déjà été souligné à plusieurs reprises, les garçons se placent dans un rapport salarial, ils se comportent comme des quasi-professionnels et axent davantage leurs critiques sur l'aspect contractuel de leur rapport à l'entreprise (conditions de travail, contrat d'apprentissage, etc.). La souffrance éthique est dès lors dicible, puisqu'elle s'inscrit dans 
le prolongement d'une revendication à pouvoir exercer son métier dans les règles, de pouvoir prétendre à une certaine éthique professionnelle.

Compte tenu de ce qui vient d'être illustré, il est particulièrement important de se questionner sur l'impact à court, moyen et long terme de la souffrance vécue durant la formation professionnelle. Dans certains cas, les jeunes se sont trouvé.e.s démuni-e.s face à ce qu'elles et ils ont découvert du fonctionnement de l'entreprise. Confronté.e.s pour la première fois au monde du travail, au monde adulte, leur expérience ne correspond pas à la vision qu'elles et ils avaient de la profession choisie. Pratiques frauduleuses ou illégales, concurrence déloyale, clientèle méprisée, autant de conduites qui portent atteinte à leur idée d'une éthique professionnelle. Face à ces situations, ces jeunes se sentent impuissant.e.s. Outre un sentiment de malaise, certaines situations de souffrance éthique peuvent engendrer une forme de désenchantement, de désillusion. Cela a un impact sur la motivation à poursuivre sa formation ou encore son entrée dans le monde adulte. A long terme, on peut craindre que ces futur.e.s professionnel.le.s intériorisent, banalisent ou encore reproduisent ce qui a généré de la souffrance, ou qu'au contraire, lorsqu'elles et ils ont arrêté pour rester en accord avec leurs valeurs, elles et ils portent une attention particulière à l'éthique professionnelle, aux relations, au rapport à la clientèle. Dans un sens comme dans l'autre, il semble que l'expérience de la souffrance fasse ainsi partie intégrante de la formation professionnelle.

\section{La souffrance physique}

Ces dernières années, la souffrance physique a curieusement été dissimulée par une attention accrue portée à la souffrance mentale. En effet, une part importante de la notion de "progrès» au travail, portée par les syndicats, la médecine et l'hygiène du travail, puis par l'ergonomie, a consisté depuis la fin du XIX à éliminer la pénibilité physique. Les évolutions technologiques des modes de production ont certes contribué à la diminution de la souffrance physique, mais elle est toujours présente sous de nouvelles formes (Dejours, 2000b; Paugam, 2003). On assiste notamment à l'augmentation des troubles musculo-squelettiques liés à l'intensification du travail (Dolivo et Rosende H., 2000). Par ailleurs, la souffrance physique provient également des situations de prise de risque, de mise en danger ou de mise à l'épreuve (Dejours, 1998; Legault, 2001). La mise en danger de soi ou d'autrui est assez fréquente dans le monde ouvrier, où elle relève paradoxalement de stratégies collectives pour supporter le danger. On pense notamment aux ouvriers qui ne respectent pas 
les consignes de sécurité, font preuve d'attitudes bravaches et prennent des risques qui paraissent inconsidérés (Dejours, 1998). Quant à la mise à l'épreuve, qui est le pendant de la mise en danger, mais sur autrui, elle fait parfois partie intégrante d'une socialisation professionnelle, qui se ferait sous forme de bizutage.

La question dela souffrance physique apparaît dans huit entretiens. Dans certains cas, il s'agit de souffrance liée à la pénibilité intrinsèque du métier, aux conditions de travail. Cette pénibilité se marque sur les corps encore jeunes, pas habitués, comme le relate Massimo, un carreleur de 17 ans:

Physiquement, faut suivre. Pis faut être motivé, vachement motivé parce que le matin, après la journée qu'on a eue le jour d'avant, faut bien récupérer la nuit. Ouais, porter toute la journée des sacs de colle au $5 e$ étage quand y a pas d'ascenseur! Pis sinon, euh, porter les carreaux, mais toujours sans ascenseur, physiquement c'était dur, pis c'était lassant. Parce que je sais pas, y prennent les apprentis, y nous prennent un peu comme leur chien.

La pénibilité, et donc la souffrance physique, s'atténuera peut-être avec le temps, lorsque l'apprenti.e sera sorti.e de son statut d'infériorité. Mais ce n'est pas sûr, dans la mesure où elle peut être directement rattachée au «simple» exercice du métier, impliquant par exemple des charges lourdes, des horaires contraignants, un travail en plein air exposé aux aléas des conditions météorologiques, etc. Et de toute façon, le temps ou l'habitude n'auront parfois jamais l'occasion de faire leur œuvre dès lors que la souffrance physique aura déjà conduit certain.e.s jeunes à rompre leur apprentissage. C'est le cas notamment de certaines jeunes femmes en situation pionnière, la douleur physique et l'épuisement ayant eu raison de leur motivation à poursuivre leur parcours atypique. Par exemple Anne-Cécile, une paysagiste de 17 ans, dont l'expérience n'a pas résisté à l'épuisement physique:

Moi, j'amenais les brouettes de béton à la main et pis lui [le patron], ben il posait les pierres quoi, pis toute la journée je faisais ça, donc ça va un moment de poser du béton, pis le lendemain, c'était les cailloux, après fallait faire un mur de nouveau, ramasser la terre, c'était beaucoup trop physique. Pis je faisais ça toute la journée, c'est le soir surtout, tu sens ton dos en arrivant, il fait mal et le lendemain il faut recommencer... 
Les accidents du travail sont heureusement rares dans ces témoignages. Parfois ils sont liés à l'usage de machines, comme on l'a vu précédemment pour Jacques, un menuisier de 18 ans qui a "eu deux accidents avec les machines"; d'autre fois, ils sont provoqués par les rythmes de production, par les cadences ou par une culture du métier qui valorise la prise de risque ou minimise les consignes de sécurité.

Enfin, la souffrance physique témoigne aussi de dysfonctionnements relationnels et organisationnels, la mise en danger ou la soumission à des efforts particulièrement violents fonctionnant comme de véritables mises à l'épreuve. Les personnes qui en sont victimes doivent prouver qu'elles appartiennent bien à un collectif de travail. Ces situations interviennent dans des environnements relativement hostiles, par exemple réfractaires à l'arrivée d'une pionnière. Les agressions qu'elles subissent peuvent être considérées comme relevant del'ordre de la violence (Legault, 2001). Agnès, une peintre en carrosserie de 18 ans, se retrouve dans une situation de mise à l'épreuve physique, après une blessure de snowboard:

J'étais à l'assurance une semaine parce que j'arrivais plus à plier le genou, j'avais mal, j'ai dû faire les piqûres de cortisone, le lendemain j'ai dû aller bosser, j'arrivais plus à me plier, j'arrivais plus à me baisser et bien sûr il [le collègue] le savait très bien et il faisait tout pour me faire baisser et tout ça, pis le jour après j’ai été chez le médecin.

\section{a) Les limites du corps dans les métiers masculins}

La souffrance physique est relevée - dans 7 cas sur 8 - dans des métiers masculins, elle révèle ainsi des logiques de genre. En effet, sur les quatre filles évoquant de la souffrance physique, trois sont des pionnières. Il s'agit d'une peintre en carrosserie, d'une paysagiste et d'une cuisinière. En revanche, aucun des quatre garçons qui font référence eux aussi à la pénibilité physique de leur travail n'est pionnier. Il s'agit donc là d'un premier résultat à retenir, la souffrance physique est attachée, du point de vue des apprenti.e.s, principalement à l'exercice de métiers masculins. Ces données confirment ainsi le lien étroit, mis en évidence dans la section précédente, entre le type de souffrance et le secteur d'activité: si les métiers de service génèrent des problèmes d'ordre éthique, les professions traditionnellement masculines engendrent de la souffrance physique.

L'ancrage de la souffrance physique dans un contexte de travail masculin nécessite de différencier les témoignages des garçons de ceux des pionnières, soit de voir en quels termes ils et elles parlent respecti- 
vement de cette souffrance. Elle ne prend pas le même sens selon que le sexe du métier correspond à leur identité sexuelle (les garçons) ou n'y correspond pas (les pionnières), selon qu'ils sont en situation de «normalité» ou qu'elles «transgressent».

Pour l'essentiel, il ressort de l'analyse que les pionnières déplorent avant tout les effets de la pénibilité du travail sur le corps, tandis que les garçons mettent l'accent sur les conditions de travail qui les conduisent à souffrir dans leur corps. Ainsi, les filles parlent deux fois plus qu'eux de la douleur et de la fatigue qu'elles éprouvent, par exemple Sylvie, une spécialiste en restauration ${ }^{85}$ de 16 ans, et la seule femme de ce groupe a n'être pas une pionnière, le fait en ces termes:

Je faisais la cave [descendre les caisses de bouteilles], mais qu'ils [les collègues masculins] la remonte, parce que mon dos... j'marchais plus, c'est vrai c'était pénible, ça!

Parallèlement, les garçons renvoient leur souffrance physique à la question du statut et des conditions de travail, ce dont les jeunes femmes ne parlent pas du tout ici. Frédéric, un cuisinier de 18 ans, dénonce par exemple ses conditions de travail:

Ils me faisaient travailler plus, ils m'donnaient pas [...] mes deux jours de congé par semaine. Pis, ils me faisaient travailler neuf jours de suite. [...] Moi, j'faisais plus ou moins 60 heures par semaine, eux, ils en faisaient 42 .

Les filles évoquent donc la fatigue, la pénibilité, les charges trop lourdes ou la douleur, pendant que les garçons font valoir leurs mauvaises conditions de travail. Pour eux, la pénibilité est aussi liée aux charges, mais dans ce cas ils déplorent surtout l'absence de moyens techniques qui permettraient de les alléger (par exemple ils se plaignent lorsqu'il n'y a pas d'ascenseur), et ils attribuent la pénibilité à l'organisation du travail, aux horaires, aux risques d'accident ou à leur statut d'apprenti.

Par ailleurs, les pionnières sont confrontées à la rudesse de leurs pairs, parfois à des comportements de violence. Si la violence est source de souffrance mentale, elle rend la souffrance physique insurmontable.

85 Spécialiste en restauration est la nouvelle appellation de la profession de serveur/serveuse. Le métier est mixte, même si une majorité de jeunes femmes y sont engagées. Profession de service, elle est également connue pour sa pénibilité: «les spécialistes en restauration travaillent debout et se déplacent beaucoup pendant le service. [...] Leur horaire de travail est irrégulier» (http://www.orientation.ch/dyn/1109.aspx ?data=description\&id=253). 
Alexandra, une cuisinière de 22 ans, met bien ces deux types de souffrance dans la balance:

C'est déjà très dur physiquement comme métier, si derrière moralement c'est lourd... Je vois, on [ses collègues masculins] est tout le temps en train de rigoler [à son encontre], on est en train de faire les fou-fous et puis ça, ça y va!

On peut voir les mises à l'épreuve auxquelles cette pionnière est confrontée comme de véritables pratiques d'exclusion. Ou alors, elles visent à tester la capacité de la personne en situation atypique à s'intégrer dans un collectif de travail de «l'autre sexe» et à adopter ses normes et ses valeurs, y compris quand elles sont sexistes. Quoiqu'il en soit, de tels bizutages peuvent servir à enseigner la virilité défensive (voir Chapitre 7): comme les garçons, les pionnières dans des métiers masculins doivent apprendre la peur et la douleur, pour pouvoir les dominer ou les nier, évitant ainsi de mettre le collectif en danger (Dejours, 2000b; Molinier, 2000). Pourtant, dans le cas d'Alexandra, cet apprentissage sera sans doute rendu difficile par le fait qu'elle est perçue comme une fille - c'est d'ailleurs l'objet des rires à son égard. Ses collègues identifient une femme, et non un professionnel ou futur professionnel ${ }^{86}$, qui n'est pas au bon endroit ou pour reprendre les termes de Cassell: «Elle a le mauvais corps à la mauvaise place» (Cassell, 2000: 73).

La pression mise sur les pionnières explique probablement pourquoi elles ne sont pas plus prolixes dans l'évocation des souffrances physiques que génèrent les métiers masculins. Moins encore que les garçons, même si eux aussi vivent ici leur premier contact avec le monde du travail, elles sont préparé·e·s à affronter l'effort physique, la résistance, la douleur. Dans ces métiers masculins, parallèlement à la transmission des savoirs théoriques et des habiletés pratiques, il faut se socialiser «à la dure réalité du métier 〈qui rentre dans la peau〉» (G. Moreau, 2003:223). A priori, on aurait pu faire l'hypothèse que les pionnières se plaindraient davantage de la pénibilité physique liée à l'apprentissage du métier, au même titre qu'elles parlent sans détour de leurs larmes ou de leur désarroi. Mais sans doute s'interdisent-elles de mettre trop en avant leurs limites en raison du caractère transgressif de leur insertion professionnelle. Si elles le faisaient davantage, elles risqueraient de conforter les résistances de leur entourage familial, d'attiser les moqueries ou les agressions de

86 Le masculin est utilisé volontairement en référence à Cassell (2000). Elle cite une chirurgienne qui précise que ses collègues l'enferment dans une impasse: elle n'est soit pas une femme, soit pas un chirurgien. 
la part des apprentis, voire de se faire exclure du collectif de travail. Adopter la stratégie virile du non-dit ou du déni serait alors pour elles une manière d'éviter de tels risques.

Du côté des garçons, il était relativement attendu qu'ils fassent peu mention des difficultés physiques auxquelles leur métier d'homme les confronte. Ils se doivent d'être forts physiquement (Eckert et Sulzer, 2007 ; Hirata et Kergoat, 1998; Molinier, 2000). Le déni de la souffrance physique s'inscrit donc bien dans leur socialisation. Mais la manière dont quatre d'entre eux en parlent s'inscrit tout aussi bien dans ce processus de socialisation différenciée des sexes: la souffrance physique est dicible dès lors qu'ils la comprennent comme produite par le rapport de travail. Ce qui les fait souffrir, ce sont les injustices d'un rapport de travail où ils occupent un statut inférieur, où ils sont assignés à des tâches ingrates, où ils sont exploités (non-respect des horaires, des vacances). Alors même qu'ils sont encore en formation, ces jeunes hommes se placent d'emblée dans un lien salarial et «se positionnent dans une situation de travail dont ils jugent les conditions. Ce sont alors les horaires, le trop d'heures qu'ils dénoncent» (G. Moreau, 2000: 79). L'organisation du travail est au centre de leurs récriminations (Molinier, 2000). Ce positionnement apparaissait déjà dans leur manière de faire état des raisons les ayant conduits à arrêter leur formation (voir Chapitre 5), ainsi que dans leurs témoignages concernant les problèmes éthiques. La façon d'évoquer la souffrance physique se calque donc sur cette manière générale de se positionner face à l'activité. Ce ne sont pas leurs faiblesses (fragilité, incapacité physique) ou leurs manques (de force, d'endurance) qui font souffrir les apprentis, mais les conditions de travail qu'ils subissent. En d'autres termes, ils réagissent en tant que professionnels, ils affirment leur droit au travail et leur légitimité à être traités comme des travailleurs. Il va de soi, pour eux, que la formation professionnelle s'inscrit directement dans leur futur professionnel, et sans doute plus largement dans leur vie d'homme responsable, futur pourvoyeur principal de leur famille (Baudelot et Establet, 1992b; Imdorf, 2007a). La socialisation entretient ainsi «des destins de sexe qui rendent évident le travail des hommes, et donc leur formation professionnelle, et contingent celui des femmes» (Maruani, 1985 citée par G. Moreau, 2000 : 77).

L'analyse de la souffrance dans les métiers masculins fait ressortir deux éléments extrêmement intéressants du point de vue du genre. D'une part, ces professions engendrent de la souffrance physique non seulement chez les garçons, mais aussi chez les filles qui se sont «risquées » à entrer dans un bastion masculin. C'est donc bien le secteur qui 
engendre un type de souffrance particulier. D'autre part, elles socialisent les personnes en souffrance à des stratégies de déni, de silence, qui leur font taire ce qu'elles ressentent. Là encore, c'est le secteur d'activité qui produit ce type de stratégie, et celle-ci est utilisée tant par les filles que par les garçons, même si c'est pour des raisons différentes.

\subsubsection{Une souffrance propre aux apprenti·e.s}

Quelle soit mentale ou physique, la souffrance fait partie de l'expérience de la formation professionnelle, elle marque cette-dernière, faisant découvrir aux apprenti.e.s la pénibilité de certaines activités, la dureté des processus de production, mais aussi la rudesse des relations au travail, la violence des hiérarchies. Au terme de ces premières réflexions, on peut affirmer qu'il y a une souffrance spécifique des apprenti.e.s. Aux éléments déjà évoqués (pénibilité, contraintes organisationnelles, relations), s'ajoutent des contraintes spécifiques à la formation professionnelle (voir Chapitre 7). Lors de cette première confrontation au travail, leur âge, leur inexpérience rend les apprenti.e.s particulièrement fragiles. Mais avant tout c'est leur statut qui rend leur souffrance particulière: occupant la position subalterne dans l'entreprise, les apprenti.e.s ne sont fréquemment pas intégré.e.s dans l'équipe. Cette position se traduit souvent par la délégation du «sale boulot» (Hughes, 1996), l'obligation de tout accepter, mais aussi la confrontation aux défenses des travailleurs et travailleuses adultes (en ce qui concerne le statut d'apprenti.e et ses contraintes particulières, voir Chapitres 5 et 7). L'expérience en formation professionnelle engendre de la souffrance: celle du métier qui entre (G. Moreau, 2003), qui marque les corps, mais aussi celle du travail et de ses règles qui doivent être incorporées (P. Kergoat, 2006) et qui marquent les esprits. A côté de cela, un autre apprentissage se fait au travail, celui des normes de genre. Un certain nombre d'éléments de ce chapitre ont souligné le caractère genré de la souffrance, mais la violence de l'apprentissage des normes de genre, et la souffrance qui en découle, sera traitée de manière plus détaillée dans le Chapitre 8.

\subsubsection{Les souffrances spécifiques des pionnières}

Dans ce corpus, seuls deux garçons sont pionniers et n'expriment aucune souffrance, même s' ils font état d'un certain malaise (voir Chapitre 5) qui renvoie à leurinsertion particulière (absence de perspectives, monotonies des tâches). Cette absence de tout témoignage de souffrance suggère qu'ils rencontrent peu de problèmes dans leur confrontation à un uni- 
vers professionnel féminin. C'est là un phénomène connu: les hommes insérés dans des métiers de l'autre sexe sont généralement bien accueillis (Croisier, 2002 ; Guichard-Claudic et al., 2008; Imdorf, 2004; Thiébaud, 2004) et y font fréquemment un autre parcours que leurs consœurs (Le Feuvre et Laufer, 2008). Au contraire, les pionnières paient souvent le prix de leur transgression (Guichard-Claudic et al., 2008; Marry, 2007), phénomène qui se retrouve ici. En effet, sur les 7 pionnières interrogées, 6 font état d'une ou plusieurs formes de souffrance ${ }^{87}$. La présente section leur consacrera donc une analyse particulière.

Le fait qu'une très nette majorité des pionnières parlent de leurs souffrances s'inscrit dans le prolongement des réflexions de Couppié et Epiphane (2007). Ils rapportent en effet que les pionnières sont tendanciellement plus insatisfaites de leur situation de travail et souffrent davantage que les femmes insérées dans des emplois traditionnels pour leur sexe.

L'évocation systématique de souffrances, en particulier mentales, par les pionnières révèle un environnement de travail inhospitalier, voire hostile. Agnès, une peintre en carrosserie de 18 ans qui, précédemment, s'était essayée à la profession de pâtissière, une autre profession atypique, ressent même cette hostilité comme explicitement dirigée contre les femmes:

J'avais retrouvé une place bien [...] pis c'était des vrais connards là-bas, alors eux, alors c'est la même chose qu'en peinture, c'est des machos, ils aiment pas les apprenties. [...]

Des fois j'en avais ras-le-bol, j'avais envie de me casser, quoi. J'attendais que la fin de la journée parce que je crois quej'allais les tuer, quoi. [...] Jusqu'au jour où j'ai pêté un plomb quoi, c'est vrai une fois je leur ai dit: «C'est pas parce que je suis une fille, que je vais pas mieux bosser que vous", j'sais pas trop quoi, ça a rien à voir...

87 La seule qui n'exprime aucune souffrance est une fille en situation pionnière, c'est-à-dire l'une des deux jeunes femmes à être insérée dans une profession traditionnellement féminine mais dans un environnement non-mixte. Sa situation est donc différente de celle des pionnières qui sont insérées dans des professions traditionnellement masculines, mais comme signalé dans le Chapitre 4, une analyse distincte ne peut pas être proposée pour ces deux types de situations pionnières vu le nombre restreint de personnes concernées. 
Cet extrait montre que ce n'est pas le métier en soi qui fait souffrir la jeune femme, mais bien son environnement de travail, dans lequel elle subit sexisme et mise à l'écart de la part de ses collègues, jusqu'à ne plus supporter le machisme ambiant. Elle en souffre d'autant plus qu'elle a déjà vécu la même chose auparavant, lorsqu'elle faisait une autre formation atypique. Son statut doublement fragile d'apprentie et de pionnière la rend particulièrement sujette aux remarques et la place dans une position où elle ne peut pas se défendre. Bien qu'elle essaie de tenir bon, le découragement l'envahit. Soulignons ici que sa souffrance s'exprime de manière assez frontale: "j'avais envie de me casser», "j'allais les tuer», «j'ai pété un plomb». Contrairement à d'autres jeunes femmes citées précédemment et qui font montre de leur peine et parlent de leurs larmes, cette peintre en carrosserie a, semble-t-il, adopté un mode propre à son cadre de travail masculin pour s'exprimer. On pourrait l'interpréter comme une réappropriation des stratégies viriles utilisées dans ces contextes de professions masculines très rétives à l'entrée des pionnières (voir Chapitre 7).

Un autre résultat à relever est que la souffrance mentale intervient dans certains cas comme le prolongement d'une souffrance physique. En effet, les trois pionnières éprouvant de la souffrance physique font également état de souffrance mentale, à l'instar de Anne-Cécile, une paysagiste de 17 ans:

Le matin, je pleurais avant d'aller au travail tous les jours [...] j'avais vraiment envie de changer, euh changer d'orientation, pis de boîte en fait, parce que rien que le fait de me retrouver dans ce dépôt le matin-là, de voir les briques, je savais ce qui m’attendait, ça me déprimait.

Bien qu'initialement le métier et l'entreprise lui plaisaient, la pénibilité physique de l'activité est telle qu'elle a entamé sa motivation. La jeune femme commence à être démoralisée, et ce indépendamment des excellentes relations qu'elle entretient avec son formateur. La souffrance physique pèse donc fortement sur cette pionnière.

De manière générale, la façon dont les pionnières parlent de leurs souffrances laisse entendre que les normes de genre et les rappels à l'ordre sexué qui leur sont faits jouent un rôle primordial dans l'arrêt de leur formation. Il n'est certainement pas facile pour elles, qui ont fait un choix transgressif, de dire leurs difficultés, ni même de les reconnaître en leur for intérieur. Non seulement leur choix de formation correspond bien souvent à une volonté de se distancier des rôles et de la place qui leur 
sont assignés traditionnellement (Pfefferkorn, 2008), mais en outre leur entourage familial ne soutient pas toujours leur choix. Aux pressions qu'elles vivent sur le lieu de travail peuvent donc s'ajouter celles de leurs proches. Des remarques du type «on t'avait prévenue» rendraient ainsi encore plus difficile la décision d'arrêter, comme l'évoque Anne-Cécile, paysagiste de 17 ans:

Mes parents eux ils voulaient pas que je fasse paysagiste parce qu'ils savaient que ça allait finir comme ça, ils m'ont déjà prévenue: «Tu vas pas faire ça 3 ans, c'est trop physique pour toi et tout", alors j'ai paniqué au moment où j'ai décidé d'arrêter parce que eux ils allaient très mal le prendre, pis ils ont vu que j'allais pas bien pendant quelques semaines alors après ils m’ont dit: «Tu sais Cécile, si ça va pas à cause de ton apprentissage faut que t'arrêtes, ça sert à rien de te mettre, de te foutre en l'air à cause de ça», et puis là et ben vu qu'ils avaient compris je leur ai dit que...

Face à leur entourage professionnel, familial et amical, mais aussi face à un.e chercheur.e, il est donc possible que ces jeunes femmes taisent en partie leurs souffrances. L'enjeu étant de pouvoir affirmer leur capacitéà entrer dans une filière atypique et à transgresser. Dévoiler leurs propres limites dans l'exercice d'un métier masculin pourrait en effet avoir un coût identitaire important, puisque cet aveu signifierait l'abandon d'un projet professionnel parfois obtenu de haute lutte. Il signifierait également l'échec d'une affirmation de soi hors du système de genre ou malgré lui, si l'on considère comme Vouillot, que l'orientation est un outil du genre, permettant aux jeunes, au-delà d'un choix professionnel, de s'affirmer conformes, soit de se déclarer comme des filles «féminines" (ou des garçons «masculins») (Vouillot, 2002).

\subsubsection{Les multiples conséquences de la souffrance}

Les différentes formes de souffrance rencontrées lors de l'expérience en formation professionnelle ont des effets divers. La souffrance a un impact direct sur la motivation. Les apprenti.e.s évoquent en effet fréquemment le découragement, la perte d'envie de travailler comme conséquence de la souffrance. La démotivation touche de manière sensiblement identique filles et garçons, même si les jeunes femmes la mentionnent un peu plus, comme elles le font avecl'ensemble des éléments ayant trait à la souffrance. Yasmine, une assistante dentaire de 16 ans, l'exprime très clairement : 
J'avais pas l'envie d'aller en fait, le matin, pis j'étais tout le temps en souci, parce que j'avais un peu peur de mon patron.

La jeune femme établit un lien de causalité entre la peur, à l'origine de sa souffrance, et son désengagement. Dans certains cas extrêmes, la conséquence est à la hauteur de la souffrance éprouvée, et c'est un dégoût pour le métier qui s'affirme, même si l'arrêt de formation n'a pas trait au choix de la profession. Après avoir subi du mobbing, et malgré un goût appuyé pour son métier, Anaëlle, une horticultrice de 18 ans, n'arrive plus à envisager aucune profession ayant affaire de près ou de loin avec les métiers verts:

En fait dès que je vois, dans les magasins, qu' il y a des fleuristes [...] ça me rappelle des mauvais souvenirs, alors je peux pas.

De tels blocages peuvent également être liés à une souffrance physique et mentale engendrée par des accidents. C'est le cas de Jacques, un menuisier de 18 ans qui, après plusieurs accidents, a développé une phobie des machines. Il se retrouve bloqué dès lors qu'il est confrontéà une situation similaire dans un nouveau cadre professionnel:

J'ai été faire un stage de menuisier [après son arrêt de formation en menuiserie] [...] A la fin du stage, j’avais mes parents: «Vas-y accepte, accepte !» Euh, moi d'un côté je voulais pas les décevoir, j'me suis dit: «Tu devrais accepter», pis j'étais devant le patron, j'ai fait: «Non, je peux pas.»

Parfois, la démotivation dépasse le cadre de l'entreprise formatrice pour toucher également les résultats scolaires (voir Chapitre 5). Adnan, un informaticien de 18 ans, montre bien cette chaîne causale:

J'étais trop démotivé en fait. Il y a eu des moments où j'étais vraiment... je voulais pas aller au travail, ni rien, mais bon, je tenais deux jours... Et puis après, mes résultats ils ont baissé, je voulais pas aller aux cours et tout...

La perte de motivation, le désengagement, la baisse des performances scolaires apparaissent donc bien ici comme un résultat de la souffrance éprouvée durant l'expérience en formation professionnelle.

D'autres conséquences dépassent la sphère du travail et de la formation, touchant à la qualité de la vie privée et aux relations qui s'y établissent. Du côté des garçons, les expériences douloureuses vécues sur le lieu de formation professionnelle ont un impact sur leurs rela- 
tions avec leurs parents. Les filles, pour leur part, font surtout état que ce qu'elles subissent en formation professionnelle a des conséquences sur leur couple et parfois en détériore la relation. Le point de référence dans la vie privée de ces jeunes n'est donc pas le même pour les garçons et les filles: pour les premiers c'est la famille dont ils sont issus et dans laquelle ils vivent, pour les secondes c'est le couple dans lequel elles ne vivent pas (à l'exception de deux d'entre elles) mais qui semble primordial comme référent affectif.

Parfois chez ces jeunes femmes, la colère et la frustration éprouvées au travail se retournent contre le conjoint. C'est le cas pour Anne-Cécile, une paysagiste de 17 ans:

Avec mon copain, je rentrais du travail le soir et je sais pas pourquoi j'étais énervée contre lui, et je le voyais et il m’énervait...

Cela peut aller jusqu'à la violence physique (la jeune femme allant parfois jusqu'à frapper son compagnon). Insérée dans une profession atypique, elle doit gérer l'articulation entre une vie professionnelle, où elle a transgressé les frontières de genre, et une vie privée où la hiérarchie entre les sexes est restée inchangée. Il est probable que l'articulation entre vie de couple et vie professionnelle est encore plus délicate pour les pionnières. En optant pour une profession atypique, la pionnière refuse de se plier à l'ordre social hiérarchique des sexes, mais ce refus ne peut pas toujours se transposer tel quel dans une vie de couple, qui plus est dans le cadre normatif de l'hétérosexualité. Notamment, le conjoint peut penser qu'il est mis en danger par la position professionnelle de sa partenaire et peut alors tenter de se prémunir contre l'éventuelle contestation de sa position de dominant dans le couple. Par ailleurs, la jeune femme est confrontée quotidiennement aux bizutages et aux stratégies défensives de ses collègues, au risque d'y recourir à son tour (à propos du recours par les pionnières à la stratégie de virilité défensive, voir précédemment et Chapitre 7). Elle se retrouve ainsi au cœur d'une tension entre identité professionnelle et identité sexuelle, rappelant ainsi les difficultés de Mademoiselle MULVIR ${ }^{88}$ signalées par Dejours (1996). Insérée dans une

88 «Mademoiselle MULVIR est une jeune fille suivie en psychothérapie par Dejours (1996). Cette dernière, refusant de répéter un destin maternel frustré (muliérité), désire accéder à un travail intéressant et qualifié, celui de technicienne-électronicienne. Dans son milieu social, il n'y a pas de femme susceptible de représenter un modèle d'émancipation professionnelle et sexuelle. Et dans son milieu de travail exclusivement masculin, elle doit consentir à se plier aux stratégies collectives de défense et aux bizutages 
profession atypique, dont elle adopte les stratégies défensives, elle connaît une crise de l'identité sexuelle. Ces situations sont assez comparables au cas des femmes en situation d'hypogamie, c'est-à-dire lorsqu'elles sont dans une position professionnelle supérieure à celle de leur conjoint (Testenoire, 2008).

Par ailleurs, pour les pionnières ayant affaire quotidiennement aux attaques sexistes de leurs collègues, voire au harcèlement sexuel, il peut être difficile de réintégrer une relation de couple hétérosexuelle sereine. Elles doivent gérer deux sphères aux discours en apparence antagonistes: au travail, elles subissent la violence du rappel de la hiérarchie entre les sexes, sans cesse renvoyées à leur position dominée. A la maison, ou parfois avec les collègues hors travail (voir Chapitre 8), elles doivent entrer dans le jeu de la complémentarité et de la convivialité, dicté par la norme hétérosexuelle. L'hétérosexualité, comme le genre, se «joue» en effet en permanence: «la plupart des gens 〈font le genre〉 et 〈font l'hétérosexualité> tous les jours, sans réfléchir de façon critique sur ce qu'ils font. Cela s'opère par la parole et par l'action, par les pratiques d'habillement et les attitudes en général, par la participation active à des arrangements institutionnels formels, et par toutes les activités banales qui ordonnent nos vies quotidiennes" (Jackson, 2009: 30). Cette mise en scène est certainement plus importante dans des situations de transgression que dans celles où les arrangements de sexe sont respectés. C'est ce que l'on peut supposer à voir la violence de la réaction de la pionnière susmentionnée face à son petit ami, il s'agirait là d'une réponse à la réassignation à laquelle leur situation de couple la renvoie.

Parfois, les pionnières en couple jouent de l'ambivalence qui lie leur position de transgression au niveau professionnel à l'arrangement hétérosexuel formalisé dans leur vie de couple. Julie, une paysagiste de 16 ans ayant subi du harcèlement sexuel au travail, a ainsi opté pour une stratégie entièrement basée sur l'ambiguïté des rapports de genre dans lesquels elle s'inscrit. Elle informe son petit ami des nombreuses attaques à connotation sexuelle qu'elle subit dans son travail et le sollicite ce faisant pour la soutenir:

Alors ça, quand il y en a un qui a un peu été un peu trop loin, ben j’ai donné le numéro à mon copain pis il l'appelé, il lui a dit: « Si tu dis encore qu't'as envie de... », parce qu'il me disait

construits par les hommes, au risque d'une virilisation. En découle «une crise de l'identité sexuelle. [...] Entre virilité et muliérité, l’identité sexuelle des femmes qui accèdent à des métiers traditionnellement masculins se joue sur une corde raide» (Molinier, 2004: 87). 
qu'il avait envie de me faire l’amour... [...] Pis il lui a dit: « $\mathrm{Si}$ tu lui dis encore une fois ça, je te jure, je viens te casser la gueule hein.» Et pis ben depuis ben voilà.

En procédant de la sorte, la jeune femme a non seulement cherché à trouver une issue à sa souffrance au travail, mais a réaffirmé sa position dans les rapports sociaux de sexe. Bien qu'ayant transgressé les normes de genre en choisissant une profession atypique, elle est bien une femme "féminine», qu'il faut protéger. De son côté, en menaçant d'intervenir physiquement, le petit ami de cette pionnière prend certes sa défense, donne une réponse à sa souffrance, mais il réaffirme lui-aussi sa position de dominant dans la hiérarchie entre les sexes. En effet, informé par sa compagne de ce qu'elle subit au travail, il découvre une violence de la hiérarchie entre les sexes à laquelle il n'a peut-être jamais été confronté (étant donné sa place habituelle de dominant) et peut se sentir menacé (puisque cette place n'est généralement pas remise en cause dans le cadre de son couple).

\subsection{A l'origine de la souffrance, la division sexuelle du travail}

L'analyse de la question de la souffrance en formation professionnelle dans une perspective de genre laisse apparaître deux éléments transversaux, qui expliquent pourquoi, derrière des situations apparemment similaires, des différences apparaissent. D'une part, la socialisation différenciée selon le sexe influence ce qui est dicible ou non par les jeunes. D'autre part, la division sexuelle du travail, qui assigne les personnes à des activités distinctes selon leur sexe, crée des situations non identiques dans lesquelles la souffrance s'ancre sur des modes différents eux aussi.

La socialisation professionnelle vient consolider des stéréotypes incorporés tout au long de la socialisation, primaire et secondaire, et fige les normes de ce qu'est un "vrai» homme ou une «vraie» femme. Les jeunes hommes ont ainsi appris à taire leur peur, à affronter la souffrance, car « un homme, un 〈vrai 〉, ne craint pas le danger. Il se maîtrise et doit être en mesure de le prouver à tout bout de champ devant ses collègues s'il veut gagner, et conserver leur confiance» (Molinier, 2000:30). Ces stratégies de défense viriles seront discutées en détail dans le chapitre suivant, mais gageons déjà qu'elles déterminent non seulement le rapport aux pairs et aux collègues, mais aussi le rapport aux chercheur.e.s qui les interviewent. 
Les effets de la socialisation différenciée se perçoivent également dans les récits des filles sur leur souffrance, qui en parlent avec aisance et dans des termes très explicites, en en décrivant précisément les manifestations et les symptômes. Les propos de Leonora, une assistante dentaire de 16 ans, illustrent bien cette précision:

Mon père avait bien senti qu'j'allais pas du tout bien, pis j'parlais pas, pis j'arrivais pas à manger, pis il m'a dit : «Y a un problème? », il m'fait: «Parce que ça fait plusieurs soir que je te vois un peu triste» [...] Pis là ça a explosé, j'me suis mise à pleurer à table et pis j'lui ai tout raconté...»

Au final, l'analyse proposée dans ce chapitre parvient à dresser un tableau assez détaillé des causes de souffrance mentale, physique et éthique décrites par les filles, et des causes organisationnelles davantage évoquées par les garçons.

La division sexuelle du travail (D. Kergoat, 2000) est une cause fondamentale de ces souffrances. Du fait que femmes et hommes ne sont pas engagé.e.s dans les mêmes professions (ségrégation horizontale), les contraintes particulières qui pèsent respectivement sur chacun des groupes de sexe diffèrent, ainsi que les compétences attendues (force physique, attention à autrui, etc.); la souffrance n'est donc pas identique (Molinier, 2000). Il en va de même dans la formation professionnelle, en amont du marché du travail filles et garçons restent dans des filières de formation largement sexuées (OFS, 2004). C'est en fonction de cette ségrégation que l'expression et le vécu de la souffrance diffèrent : les activités de service et de soins dévolues aux femmes génèrent de la souffrance éthique, les activités del'industrie, de l'artisanat ou du bâtiment réservées aux hommes, troublées parfois par quelques pionnières, génèrent de la souffrance physique. La souffrance mentale, éprouvée par une majorité de nos apprenti.e.s indépendamment de leur filière de formation, se dit de manière différente selon les positions des jeunes dans la division sexuelle du travail, c'est-à-dire ici selon leur rapport au travail. Le travail est en effet central dans l'identité sexuelle des hommes, tandis qu'il est contingent dans celle des femmes. Ceci participe très certainement à ce que les filles identifient les relations avec la clientèle par exemple comme une source de souffrance, alors que les garçons mettent l'accent sur les difficultés liées à leurs conditions de travail.

Ainsi, pour les filles, la souffrance doit être mise essentiellement en relation avec une activité qui se déroule face à une clientèle, à des patient.e.s. Giovanna, une coiffeuse de 16 ans, explique: 
Devant moi, elle [la patronne] disait presque trop merci tout ça devant les clients, mais une fois que je montais [...] elle disait aux clients, elle racontait. Enfin, elle avait pas le droit normalement.

Pour les garçons, la souffrance est avant tout à mettre en relation avec les conditions de travail ou le statut subalterne. Massimo, un carreleur de 17 ans, insiste sur le lien entre les deux éléments:

Ils prennent les apprentis [...] un peu comme leur chien. [...]

On fait tout le sale boulot [...] et puis la manutention.

A la pénibilité d'un métier du bâtiment s'ajoute le statut subalterne de l'apprenti. Cette question du statut, fréquemment évoquée par les garçons, révèle une autre forme de division du travail, qui repose sur les divers statuts en présence dans l'entreprise. Cette autre forme de division du travail organise l'activité professionnelle selon différents critères, l'âge (plus âgés vs plus jeunes), l'ancienneté (expérience dans l'entreprise) et le statut d'emploi (stable $v$ s précaire). Ainsi, par son statut particulier (entre élève et personne productive, en position subalterne, inséré.e provisoirement dans l'organisation), l'apprenti.e se retrouve toujours en position minorisée dans cette organisation et est assigné.e aux tâches ingrates. Indépendamment de leur sexe, les jeunes en formation professionnelle endossent les tâches socialement assignées aux femmes. On peut donc faire l'hypothèse que, durant leur formation, les apprenti.e.s apprennent l'ordre social qui règne dans l'entreprise et l'ordre sexué qui régit les rapports sociaux de sexe en général (P. Kergoat, 2006). Cette recherche montre que cette position dominée peut devenir source de souffrance, en particulier lorsqu'elle est vécue par ceux qui habituellement font partie du groupe dominant: les garçons. Ce contraste de positions chez un même individu permet de comprendre, en partie au moins, la place que prend la question du statut dans la souffrance évoquée par les jeunes hommes : ils supportent difficilement d'être - provisoirement - situés dans la hiérarchie sociale comme appartenant au genre féminin (voir Chapitre 8). Dans ce processus où les tâches effectuées définissent le statut hiérarchique de la personne (Delphy, 2001; Devreux, 1992), les garçons se voient destitués des avantages attachés à leur statut sexuel, dominant dans d'autres contextes que celui de leur formation. Il s'opère pour eux une forme de déclassement, dont rend compte par exemple Patrick, un réparateur automobile de 16 ans: 
J'étais balayeur, enfin, je nettoyais le garage, simplement [...] J'avais un peu l'image de la première année au balai, la deuxième, travailler sur les roues et pis la troisième, faire le moteur, ouais. Puis en fait, ben, moi, j'ai fait que la première année, donc j'ai fait que du balai.

Le système de genre, qui se matérialise à travers la socialisation différenciée des sexes et la division sexuelle du travail, a donc un impact direct sur les divers aspects de la souffrance vécue par les apprenti.e.s. Présente tant dans l'expérience masculine que féminine, elle ne prend pas sa source dans le même contexte, elle n'est pas expérimentée de la même manière par les unes et par les autres, elle n'est pas retransmise selon les mêmes modes. Enfin, l'analyse de la souffrance sous l'angle du genre permet de faire apparaître, dans un certain nombre de cas, les tensions existant entre normes de genre et normes professionnelles. En premier lieu, lorsque des jeunes femmes entrent dans des professions traditionnellement investies par les hommes. Deuxièmement, lorsque les jeunes hommes contestent le statut qui leur est assigné, refusant l'ordre social du travail qui les place en situation de dominés, une " position subalterne [qui] ne fait pas partie de leur identité de garçons» (Devreux, 1992). 


\section{Chapitre 7 \\ Des stratégies pour faire face à la souffrance}

La psychodynamique du travail place la souffrance au travail au centre de son analyse (voir Chapitre 6), mais elle s'intéresse aussi aux stratégies mises en œuvre par les travailleuses et travailleurs devant faire face à la souffrance, aux ressources mobilisées pour affronter les contraintes des situations de travail (Dejours, 1998). Comme évoqué au chapitre précédent, cette approche a été retenue car elle permet d'analyser les stratégies non pas uniquement en tant que ressources individuelles, mais également comme des défenses construites collectivement dans un cadre professionnel.

Or, les travaux sur les ruptures de formation n'ont guère abordé la question dela souffrance, si ce n'est en termes de conséquences engendrées par la résiliation du contrat. Dès lors, ils n’ont pas cherché à investiguer les stratégies mises en place pour faire face aux difficultés rencontrées au travail. Les quelques incursions faites par les chercheur.e.s dans ce domaine se sont attachées à un événement ponctuel: la résiliation du contrat $^{89}$. Or, dans cette recherche, l'arrêt de formation est compris comme un processus marqué par une multitude d'événements auxquels les apprenti.e.s font face. Cette acception del'arrêt en termes de processus implique d'identifier les stratégies mises en œuvre tout au long de leur (bref) parcours dans une première formation professionnelle; surtout, elle incite à répertorier la diversité de ces stratégies. Ce faisant, on peut espérer comprendre pourquoi certain.e.s se maintiennent dans le circuit du marché des places apprentissages tandis que d'autres le quittent.

Les nombreuses recherches sur les stratégies de défense proposées par la psychodynamique du travail offrent des pistes pour analyser la rupture d'apprentissage en termes de processus. A partir de ces recherches généralement menées avec des adultes, le présent chapitre montrera que les jeunes mobilisent toute une série de stratégies qu'utilisent également leurs aîné.e.s. Par ailleurs, les jeunes en développent d'autres quileur sont propres, typiques de leur jeune âge, de leur méconnaissance du monde

89 Par exemple l'étude LEVA (Stalder et Schmid, 2006a), celle de Ferron et al. (1997), et la recherche de Rastoldo et al. (2009). 
du travail et de leur insertion particulière. L'accès à une population adolescente permet ainsi de poursuivre les réflexions entamées par la psychodynamique du travail. En cela, le système de formation professionnelle suisse offre un cadre d'analyse exceptionnel. Il permet d'examiner la situation de jeunes inséré.e.s dans le monde du travail, alors que dans la plupart des pays occidentaux, les adolescent.e.s suivent majoritairement une formation scolaire. En outre, généralement seul.e.s les jeunes des classes populaires sont concerné.e.s par une insertion précoce dans l'emploi (P. Kergoat, 2006; Nicole-Drancourt et Roulleau-Berger, 2002), tandis qu'en Suisse, la formation professionnelle - accueillant près des $2 / 3$ des jeunes du pays - touche une population plus large. Elle concerne ainsi les enfants de milieu ouvrier, celles et ceux dont les parents sont des employée.e.s, des cadres intermédiaires ou des indépendant.e.s, mais aussi parfois des jeunes issu.e.s de milieux intellectuels et plus rarement des jeunes issu.e.s des milieux dirigeants.

Si certains des travaux de psychodynamique du travail adoptent une perspective de genre (Molinier, 2004) ou relèvent le caractère sexué de l'activité (Dejours, 1998), ils le font en analysant une seule profession, par exemple l'activité des puéricultrices (Molinier, 2002b), des infirmières (Molinier, 2000), des enseignant.e.s (Maranda et Viviers, 2011), des employés de centrale nucléaire, des pilotes de chasse ou des travailleurs du bâtiment (Dejours, 1998, 2000b). L'un des intérêts de la présente recherche est de proposer un regard transversal qui englobe diverses professions représentatives de l'ensemble des secteurs d'activité. Cette perspective permet une comparaison entre femmes et hommes qui tienne compte des caractéristiques particulières et sexuées de leurs insertions professionnelles respectives. Elle permet également d'examiner quels sont les effets de la réalité professionnelle ségréguée sur les stratégies adoptées par les jeunes, au sortir d'un secondaire fréquemment mixte. Nouvellement plongé.e.s dans un environnement organisé autour de la division sexuelle du travail, dans quelle mesure leur est-il possible de recourir par exemple à des stratégies de genre, la virilité (Dejours, 1998; Molinier, 2000) ou la muliérité (Molinier, 2000, 2004)? Ces stratégies sont-elles connues des apprenti.e.s ou font-elles partie de ce que ces jeunes apprendront au cours de leur socialisation professionnelle, en étant confronté.e.s à des collègues et à des collectifs de travail? 


\subsection{Souffrance des apprenti-e·s et réponses spécifiques}

Comme l'a illustré le chapitre précédent, il existe une souffrance spécifique, que les jeunes expérimentent lors de leur passage en formation professionnelle. Le métier qui entre dans la peau est une souffrance, les futur.e.s professionnel-le.s apprennent la pénibilité, les horaires, les cadences, les relations de travail, la hiérarchie, mais elles et ils apprennent aussi les normes de genre. Et cet autre apprentissage est lui-aussi douloureux.

\subsubsection{Retour sur les contraintes de la formation professionnelle}

La souffrance éprouvée durantl'expérience de la formation professionnelle est à mettre en relation avec les contraintes spécifiques de cette filière (Lamamra et Masdonati, 2011b). Le recours à la psychodynamique du travail a permis de reconstituer ces contraintes à partir des récits des jeunes. Contrairement à ce qui se fait généralement dans les interventions en psychodynamique, le travail n'a pas pu être observé in situ. Les analyses montrent cependant que les contraintes caractérisant la formation professionnelle en alternance se centrent sur la particularité du statut d'apprenti·e, sur certaines spécificités de l'insertion dans l'emploi, sur l'organisation du travail et sur le contexte dans lequel s'inscrit ce système de formation (Probst, 2004).

\section{Le statut d'apprenti.e}

La particularité du statut d'apprenti.e est qu'il se compose de rôles ambigus, parfois antagonistes. Ces jeunes ne sont plus des élèves, puisqu'elles et ils ont fait le choix de quitter le monde scolaire «traditionnel», mais restent tout de même des personnes en formation. Bien qu'inséré.e.s dans le monde du travail, ces jeunes en apprentissage ne sont pas censé.e.s être des travailleurs ou des travailleuses. Plus tout à fait élèves, pas encore des professionnel-le.s: l'ambiguité est difficile à gérer, tant pour les jeunes que pour les collègues et la hiérarchie. De fait, ces jeunes en formation oscillent entre trois rôles : celui d'apprenti.e, censé.e apprendre un métier en le faisant; celui de "pommeau ", chargé.e des tâches subalternes (rangement, nettoyage); et celui de main d'œuvre bon marché, participant à la production. Ce statut a mbigu place souvent l'apprenti.e face à une tension entre apprendre et produire (G. Moreau, 2000), une tension qui peut être source de souffrance et qui représente une contrainte spécifique de la formation professionnelle. A cela s'ajoute la position de 
l'apprenti.e, qui, en bas de la hiérarchie de l'entreprise, est en situation de vulnérabilité. Cette position se traduit dans la division du travail par une assignation fréquente aux tâches d'entretiens, au "sale boulot» (Hughes, 1996; Lhuilier, 2005). Ce renvoi à des tâches connotées comme féminines signale la position dominée des apprenti-e.s, assimilé.e.s, le temps de leur formation, à une position féminine dans les rapports sociaux de sexe (voir à ce propos Chapitre 8).

\section{L'insertion dans l'emploi}

En principe, la formation professionnelle en alternance permet une entrée progressive sur le marché du travail (Cohen-Scali, 2000). Pour autant, il ne s'agit que des prémisses du processus, puisque la formation professionnelle ne dure qu'entre deux et quatre ans. De plus, si, traditionnellement, les apprenti-e.s en fin de formation pouvaient espérer être engagé.e.s dans l'entreprise formatrice, ce n'est plus systématiquement le cas aujourd'hui (G. Moreau, 2000). Le temps de la formation professionnelle est davantage une insertion provisoire ou partielle dans l'emploi, cette insertion n'étant vraiment aboutie que lorsque l'apprenti.e entre sur le «vrai » marché du travail, à l'issue de la formation. Or, ce passage dans l'entreprise limité dans le temps, ajouté à la question du statut évoquée ci-dessus, rend difficile l'accès au soutien d'un collectif de travail, à ses solidarités, à ses connaissances des stratégies de défense ou encore aux savoir-faire d'une communauté de pratiques (Lave et Wenger, 2002).

\section{L'organisation du travail}

Le principe de la division du travail traverse l'ensemble des activités productives et reproductives. Il s'organise à différents niveaux : entre les régions (division internationale du travail, cf. Fontagné, 2007), entre les sexes (division sexuelle du travail, cf.D. Kergoat, 2000), ou entre les divers statuts d'emploi (P. Kergoat, 2003). Le statut d'apprenti.e et sa position dans la hiérarchie del'entreprise conduisent fréquemment à une division entre jeunes travailleurs ou travailleuses et collègues expérimenté.e.s (Chaix, 1996). Aux apprenti.e.s incombent les tâches ingrates, les tâches d'entretien et, parfois, les tâches pénibles. Cette division du travail les place non seulement en situation de vulnérabilité (abus, exploitation, violences), mais encore ne leur offre guère d'autonomie. Assigné.e.s à des tâches subalternes, elles et ils ne parviennent pas à apprendre le métier, notamment la partie non prescrite du travail. Ces jeunes apprennent en revanche la division sexuelle du travail, la répartition entre les tâches nobles, liées à l'exercice du métier à proprement parler, et les tâches 
ingrates, de nature domestique ${ }^{90}$. Connotées comme «féminines», ces dernières sont réservées aux apprenti.e.s en raison de leur statut subalterne au sein de l'entreprise. Ainsi, indépendamment de leur sexe, les apprenti.e.s endossent du "genre» féminin qui consolide leur position dominée (ce qui n’a pas les mêmes implications pour les filles et pour les garçons, voir Chapitre 8). Contrairement aux travailleurs et travailleuses adultes, il ne leur est pas donné de trouver dans le travail: « la coopération, l'autonomie, l'initiative, la créativité, activités qui relèvent, de ce fait, des 〈travaux structurants et sublimatoires〉» (Hirata et Kergoat, 1998: 100). Un autre aspect qu'il s'agit de relever ici est que une partie des prescriptions adressées aux apprenti.e.s sont non-dites, les jeunes étant jugé.e-s sur leur capacité à anticiper ou à décoder ce qui est attendu en termes de corvée, notamment. Un·e bon-ne apprenti.e fait les choses (s'empare du balai, range les pièces) sans qu'on le lui demande. Ainsi, non seulement elles et ils n'ont accès qu'à la part prescrite du travail, mais encore celle-ci n'est pas toujours explicitée.

\section{Le contexte de la formation professionnelle en Suisse}

En Suisse, les jeunes optant pour la formation professionnelle doivent choisir un métier vers l'âge de 15 ans. Au-delà de la précocité du choix, celui-ci doit se faire dans un contexte particulièrement délicat (voir Chapitre ${ }^{\text {er }}$ ). D'une part, les entreprises formatrices attendent parfois de leur apprenti.e qu'elle ou il soit une force productive supplémentaire; d'autre part, elles ont souvent de la peine à décharger la personne formatrice afin qu'elle encadre et soutienne véritablement son apprenti.e; enfin, la pression au rendement qui pèse sur l'ensemble des collègues participerait à briser les solidarités au sein du collectif de travail, et, par-là, empêcherait la transmission des ficelles du métier, dont les stratégies de défense font partie.

Etroitement lié aux logiques du marché du travail, le marché des places d'apprentissage en reproduit les logiques. Les apprenti.e.s expérimentent dès lors, et déjà même avant l'entrée en apprentissage, les logiques de ségrégation et de discrimination, en outre, les filles sont confrontées à la sur-sélection (voir Chapitre ${ }^{\mathrm{er}}$ ). Confronté.e·s aux

90 Dans cette étude, un accent particulier a été mis sur l'apparentement entre les tâches subalternes et les tâches d'entretien associées aux tâches domestiques exécutées par les femmes. D’autres tâches subalternes, qualifiées de tâches de tâcheron, sont elles-aussi disqualifiées, notamment par le biais du racisme. N’y ayant guère été confrontée dans les entretiens, cette question n’a pas été retenue ici. 
logiques du marché des places d'apprentissage et du marché du travail, isolé.e.s par un statut ambigu et une insertion temporaire dans l'emploi, assigné.e.s à des tâches peu valorisantes dans l'organisation du travail, peu ou mal encadré.e.s à cause de la pression économique subie par les PME, les apprenti.e.s se retrouvent au cœur de multiples contraintes. Les stratégies présentées dans ce chapitre doivent donc être analysées dans ce contexte. Que ce soient les stratégies non retenues ou les stratégies inédites, elles doivent être lues comme les réponses de personnes ayant un statut particulier et prises dans des contraintes spécifiques.

\section{Faire face aux stratégies défensives des adultes}

Un aspect qu'il s'agit également de signaler ici, et qui n'a pas véritablement fait l'objet d'une analyse dans ce chapitre ou dans le précédent, est la confrontation non seulement que les jeunes ont avec les contraintes de l'organisation du travail, avec la pénibilité des métiers qu'elles et ils apprennent, mais aussi la confrontation aux stratégies de défense mises en œuvre par leurs collègues. Certaines situations de violence verbale, physique doivent être interprétées comme la réponse des travailleurs et travailleuses aux contraintes qu'elles et ils subissent. Leurs stratégies défensives rencontrantl'inexpérience et la fragilité des apprenti.e.s. Dans les contextes professionnels, où les stratégies défensives mettent en jeu la virilité, cette confrontation est d'autant plus brutale que les apprenti.e.s incarnent la fragilité (en termes de statut), la faiblesse, ce contre quoi ces stratégies sont dressées. Leur seule présence rappelle aux travailleurs et travailleuses adultes la pénibilité, mais aussi les risques inhérents à leur activité. Il s'agit donc de se défendre, pas seulement contre ce qui fait peur ou ce qui fait mal, mais aussi contre celles et ceux qui mettent en péril cette stratégie de déni. Comme les femmes, les apprenti.e.s suscitent alors le mépris de ceux (et parfois de celles) qui ont opté pour les stratégies viriles.

\subsubsection{Stratégies collectives, «idéologies défensives» et stratégies individuelles}

La psychodynamique du travail s'intéresse aux stratégies mises en œuvre par les travailleuses et travailleurs devant faire face à la souffrance, aux ressources qu'elles et ils mobilisent pour affronter les contraintes des situations de travail (Dejours, 1998). Les travaux en psychodynamique du travail différencient les stratégies, selon qu'elles sont mises en œuvre individuellement, collectivement ou encore qu'elles s'érigent en idéologies 
défensives. Les stratégies ne sont donc pas seulement différenciées par type (le clivage, la concurrence déloyale, etc.), mais sont distinguées entre stratégies individuelles et collectives. Ces différentes formes sont mises en œuvre en fonction des contextes professionnels particuliers, elles ne mobilisent pas les mêmes ressources ni les mêmes mécanismes. Ainsi, le secteur, l'organisation du travail ou encore le taux de mixité d'un métier joue un rôle sur les stratégies utilisées, celles-ci étant des réponses aux contraintes spécifiques que subissent les travailleurs et les travailleuses.

\section{Stratégies collectives}

Les stratégies collectives impliquent un ensemble de conduites, de représentations, de valeurs, de non-dits, mais aussi le collectif de travail, les pair.e.s. Elles s'ancrent dans certains contextes professionnels, en particulier des travaux à caractère collectif (bâtiment, travaux publics). Le travail en équipe, la participation à un groupe d'opérations dont le sens est compris par l'ensemble des collègues (et qui disparaît dans les différentes formes d'organisation scientifique du travail) rendent possible la mise en œuvre de défenses collectives (Dejours, 2000b). Ces stratégies participent à la cohésion du collectif de travail, car «travailler ce n'est pas seulement avoir une activité, c'est aussi vivre: vivre le rapport à la contrainte, vivre ensemble, affronter la résistance au réel, construire le sens du travail, de la situation et de la souffrance» (Dejours, 1998: 149). Ces défenses jouent un rôle capital dans la préservation de la santé mentale, par contre elles ont plutôt tendance à aggraver les risques engendrés par l'activité qu'à les limiter (déni de perception, risque tourné en dérision, mises àl'épreuve, etc.). Pour fonctionner, elles nécessitent la participation et l'adhésion de l'ensemble du collectif, une seule personne n'adhérant pas et c'est tout le système qui s'effondre, en laissant à la peur, au danger la possibilité de faire irruption.

\section{"Idéologies défensives"}

L'idéologie défensive est une radicalisation de la stratégie collective de défense mise en place, au départ, pour contrer la souffrance vécue au travail. Elle est élaborée par un groupe social particulier (Dejours, 2000b, illustre cette stratégie au travers de l'exemple du sous-prolétariat) de façon à valoriser un ensemble de comportements socialement acceptés construits en réaction à l'organisation spécifique du travail (Maranda, 2003). Elle émerge dans des situations extrêmes de souffrance, dans lesquelles l'enjeu - faute de pouvoir agir sur l'organisation du travail - est la cohésion du collectif. Dans ces situations, l'idéologie défensive devient 
un but en soi, et la souffrance n'est plus pensée comme conséquence du travail, mais comme le résultat d'un affaiblissement de la stratégie défensive (Dejours, 2000b). L'idéologie défensive dès lors qu'elle devient une fin en soi ne protège plus de la souffrance et ne permet pas d'agir sur les contraintes pathogènes de l'organisation du travail.

\section{Stratégies individuelles}

Les mécanismes de défense individuels font écho aux contraintes du travail répétitif, du travail à la chaîne, de certaines activités de bureau en informatique, dans les assurances ou dans les banques (Dejours, 2000b). Ces stratégies sont mises en œuvre dans des contextes de travail taylorisé, où chaque personne est isolée, l'activité morcelée. Elles mettent en évidence l'isolement des travailleurs et travailleuses et le fractionnement de l'activité. Lorsque les personnes ne bénéficient pas des formes de coopération défensives, elles sont plus fragiles, prennent davantage de risques en matière de santé mentale. Les pathologies de l'isolement sont par exemple fréquentes dans les situations où les gens ne bénéficient plus des formes collectives de défense (Dejours, 2001). Les stratégies individuelles de défense occupent une large place dans l'adaptation à la souffrance, les personnes cherchent à "faire avec", à composer avec les contraintes subies.

Compte tenu des contraintes de la formation professionnelle, les apprenti.e.s du corpus optent principalement pour des stratégies individuelles. Elles et ils ne sont pas encore intégré.e.s au collectif de travail, ne constituent pas un collectif, puisque fréquemment seul.e.s dansl'entreprise. En outre, elles et ils effectuent des tâches souvent répétitives, leur temps est fréquemment morcelé et il est défini par le travail des autres, en particulier de la personne formatrice (pour une analyse de l'activité des apprenti.e.s, voir le Chapitre 8). Inexpérimenté.e.s sur le marché du travail, elles et ils optent donc pour des stratégies qui leur permettent de s'adapter à la souffrance. La recherche d'une adaptation aux contraintes de l'organisation du travail, y compris lorsque celles-ci génèrent de la souffrance, s'explique pour cette population particulière par le fait que les apprenti.e.s ignorent fréquemment si les contraintes font partie de la norme du travail ou pas.

\section{Stratégies multiples}

Le statut ambigu d'apprenti.e, le manque d'expérience sur le marché du travail, la position dans l'organisation du travail, l'insertion provisoire sur le marché du travail et de fait dans le collectif de travail font que les jeunes 
privilégient les stratégies individuelles. Le recours à ces stratégies est de l'ordre de l'expérimentation, les apprenti.e.s n'ayant ni l'expérience, ni le soutien d'un collectifleur permettant de privilégier un système défensif plutôt qu'un autre. Les résultats mettent en évidence un nombre total de stratégies largement supérieur au nombre de personnes interviewées. De même, les entretiens laissent apparaître que quasi personne ne fait appel qu'à une seule stratégie. Il ressort donc que pour faire face à la souffrance en formation professionnelle, les apprenti.e.s recourent simultanément ou successivement à différentes stratégies. Ainsi, les jeunes activent parfois jusqu'à neuf stratégies. Précisons toutefois que les deux tiers utilisent entre trois et sept stratégies. Enfin, quatre personnes n'utilisent qu'une seule stratégie et trois n'ont même recours à aucune. Toutes ces situations sont le fait de garçons $(\mathrm{N}=7)$. Comme précédemment pour la souffrance, on peut supposer que la virilité défensive agit sur la mise en récit de soi. Ne pas signaler la souffrance, et par conséquent le besoin de mettre en place des défenses face à celle-ci, c'est faire preuve de sa capacité à faire face aux événements, et donc de sa virilité.

La diversité des stratégies retenues semble être un élément récurrent du parcours de l'ensemble des apprenti.e.s interrogé.e.s. Lorsqu'une attitude ne semble pas payante, les jeunes en adoptent une autre, puis une autre, et ainsi de suite. Il apparaît de plus que les jeunes mettent parfois en œuvre des systèmes de défense qui peuvent sembler contradictoires (réaction $v s$ non-réaction; isolement ou silence $v s$ partage). Néanmoins, selon la manière dont ces stratégies sont organisées entre elles, l'impression qui s'en dégage est très variable. Elle donne à voir des profils d'apprenti-e.s très différents, entre maîtrise et agissements paradoxaux. Les entretiens n'ayant pas suivi une véritable chronologie des faits, il est difficile voire impossible de construire un séquençage fiable des stratégies pour l'ensemble des personnes interviewées.

\subsection{Les défenses mises en œuvre}

Face aux contraintes particulières de la formation professionnelle, les apprenti.e.s déploient un certain nombre de stratégies, auxquelles s'intéressera cette section. Pour simplifier la compréhension, quatre stratégies ont été identifiées (Lamamra et Masdonati, 2008): la dérobade, l'effacement, l'accommodation et l'affirmation, dont les formes peuvent être diverses. Certaines de ces formes ont été identifiées dans la littérature, d'autres apparaissent ici de manière inédite, elles seront définies au fur et à mesure. 


\subsubsection{La dérobade}

La dérobade est un ensemble d'attitudes visant à éviter de faire face aux problèmes, à prendre une certaine distance ou à sortir du jeu. Cette stratégie peut prendre la forme du désengagement, de l'évitement ou du déni. Un effet commun de ces attitudes est un certain désinvestissement de l'identité professionnelle, qui est souvent délaissée au profit d'identités se bâtissant dans d'autres sphères de l'existence. Il serait d'ailleurs intéressant d'analyser la façon dont les comportements de dérobade sont perçus par les collègues, l'employeur.e ou la personne formatrice. Peut-être que ces personnes assimilent la dérobade à de la démotivation, alors que pour les jeunes, elle constitue une stratégie de défense. Enfin, il faut signaler que les effets de ces systèmes de défense peuvent se révéler délétères. Par exemple, le déni risque de déplacer le réel hors de la pensée, ce qui entrave l'action (Dejours, 2000b).

\section{Le désengagement}

La dérobade peut prendre la forme du désengagement (Leclerc et Maranda, 2002; Maranda et Leclerc, 2000), qui est une manière de se détacher de la sphère professionnelle, de s'en désinvestir. Cette dernière est alors soit dévalorisée, soit son importance est minimisée par rapport à d'autres sphères. Un quart des jeunes tendent à désinvestir la sphère de leur formation $(\mathrm{N}=11)$. Elles et ils en relativisent ainsi l'importance, ce qui leur permet de mieux supporter une situation difficile. C'est ce que fait Adnan, un informaticien de 18 ans:

Au début, ça allait bien et tout, puis après, quand je me suis dit: «Je laisse tomber, de toute façon.» Après, je travaillais plus, j'étais plus motivé. Il y a eu des choses que j'aimais bien, je les faisais, et puis genre les maths, les trucs comme ça scientifiques, pfft, [j’y allais] juste pour être là.

Le désengagement peut parfois être partiel et ne toucher qu'un pan de la formation duale, par exemple il n'intervient que dans la sphère professionnelle et non vis-à-vis de l'école. Tiffany, une employée de commerce de 17 ans, s'est investie dans le volet théorique lorsque la pratique a posé problème:

Côté travail, j'ai vu que ça plantait, donc j'ai laissé tomber le travail, puis j'ai fait «bon ben, je me rattrape sur les cours». 


\section{Le déni}

La dérobade peut également prendre la forme du déni (Dejours, 1998, 2000b; Leclerc et Maranda, 2002; Maranda et Leclerc, 2000), qui consiste en un évitement de la réalité. Cela peut se traduire par une banalisation, une négation de celle-ci ou par la fuite dans l'activisme. Enfin, la procrastination peut être un ressort utilisé. Malgré une réalité objectivement pénible et une réelle souffrance qui aboutissent à la rupture du contrat d'apprentissage, certain.e.s apprenti.e.s nient, ou en tout cas banalisent, la gravité de la situation qu'elles et ils sont en train de vivre, ainsi que les conséquences qu'une mauvaise résolution de celle-ci peut engendrer $(\mathrm{N}=7)$. Bastien, un monteur-électricien de 15 ans, opte pour cette forme de dérobade:

J'essaie de penser à autre chose, c'est tout. Finalement, maintenant c'est fait, c'est fait, puis voilà, quoi. Puis je me dis que de toute façon, même si je trouve pas cette année, je vais faire ce qu'il faut pour trouver l'année prochaine.

Mais le déni est difficile à mettre en place, car lorsque les problèmes sont importants, que la souffrance est forte, ce n'est pas simple de les oublier. Hakem, un réparateur automobile de 19 ans, montre bien l'effort que cela nécessite:

Jusqu'au bout, j'essaie d'oublier. Si j'arrive pas, ça reste dans la tête, mais je veux le laisser de côté et penser à autre chose.

\section{L'évitement}

Proche du déni, la dernière forme de dérobade est l'évitement. Il s'agit ici d'échapper ou d'esquiver le problème rencontré. Ce contournement peut être provisoire (absence, arrêt maladie) ou définitif (arrêt de la formation). Lorsque les difficultés se rencontrent dans l'entreprise formatrice, certaines personnes vont tout mettre en ouvre pour ne plus se rendre au travail $(\mathrm{N}=10)$. Ce comportement permet alors d'éviter la confrontation, comme le fait Daniele, un opticien de 17 ans :

J'allais pas pendant deux ou trois jours au boulot. J'étais juste mal! Et pis, heu... j'étais fatigué, le matin, j’avais pas envie de me lever pour aller travailler.

Lors de problèmes relationnels, l'objet de l'évitement peut être un·e collègue. Certain.e.s apprenti.e.s mettent en place de vrais stratagèmes pour 
ne pas avoir à travailler avec cette personne. Stella, une esthéticienne de 17 ans, arrive par exemple à repousser la rencontre avec une collègue en se faisant porter malade:

Je me suis un peu arrangée pour, parce que j'avais pas du tout envie de retravailler avec elle. Alors, j'ai été chez mon médecin pour qu'il me mette en maladie au moment où elle revenait

Avec la dérobade, on pourrait parler de stratégies de résistance, bien qu'il ne s'agisse pas d'un refus frontal d'effectuer les tâches ou d'une désobéissance aux injonctions professionnelles. Les jeunes se désengagent, s'économisent, évitent les situations qui génèrent de la souffrance.

\section{La dérobade, une stratégie marquée par le genre?}

La dérobade est une stratégie légèrement plus investie par les garçons (15 pour 13), cependant vu le faible nombre de jeunes concerné.e-s, cet écart ne permet pas une analyse particulière. Les réflexions présentées sont donc purement exploratoires. Ce qui semble par contre intéressant, c'est l'usage de conduites différentes au sein d'un même groupe de stratégies. Ainsi, les différentes stratégies face à la souffrance et à la peur se colorent selon le genre. Ces écarts sont cependant assez faibles. Dans les stratégies de dérobade, les filles choisissent plus volontiers l'évitement (6 pour 4) pendant que les garçons optent plus fréquemment pour le désengagement (6 pour 5). Quant au déni, il se détache des autres stratégies, en ce sens qu'il est nettement plus choisi par les garçons (5 pour 2). A ce titre, il sera analysé plus bas comme stratégie de genre (avec l'hypercorrection et la virilité défensive).

Ce recours, au sein d'une même stratégie, à des comportements différenciés renvoie probablement au fait que les stratégies composent avec les normes du travail, les attendus genrés du travail, mais sont également le résultat de processus de socialisation antérieurs. Les stratégies adoptées refléteraient donc une socialisation familiale et scolaire. Les filles auraient ainsi appris à éviter le conflit et la confrontation, ce notamment afin de se maintenir en formation. Parallèlement, les garçons se désengageraient plus facilement, reproduisant ainsi une attitude déjà à l'œuvre face à l'école. Ainsi, de manière inédite (grâce aux stratégies adoptées), filles et garçons renouvellent en formation professionnelle, les attendus sociaux face à l'éducation. 


\subsubsection{L'effacement}

Cette stratégie consiste à ne pas se montrer, à faire preuve de la plus grande discrétion possible. On peut parler d'un véritable effacement de l'espace social de l'entreprise. L'effacement peut prendre les formes suivantes: le silence, la non-réaction, la soumission et l'isolement. Les apprenti.e.s seraient amené.e.s à développer ces comportements du fait de leur statut ambivalent et de leur position particulière dans l'entreprise. Bien que l'effacement semble dans certains cas comprendre des défenses non nécessairement conscientes, qui fonctionneraient comme des réflexes permettant de se protéger de la souffrance et de la peur (Dejours, 2000b), ces stratégies sont intentionnelles. Se mettre en retrait, se taire, ne pas réagir sont ainsi le fruit d'une décision.

\section{Le silence}

Lorsque l'effacement prend la forme du silence (Dejours, 2000b; Leclerc et Maranda, 2002), il faut le comprendre au sens propre. Il s'agit de réprimer la parole, de ne pas dire, de ne pas dénoncer ce qui se passe. Cela autant envers l'extérieur (les parents, l'école, le réseau amical) qu'envers les collègues ou la direction. La moitié des jeunes $(\mathrm{N}=23)$ a préféré «simplement» se taire et ne pas dénoncer, ni même parler de leur souffrance, en tout cas pendant un certain temps. Il est difficile de déceler, dans les témoignages, les justifications ou les raisons profondes de cette réaction par l'introversion. Daniele, un opticien de 17 ans, a opté pour cette forme d'invisibilité sociale:

Je disais rien, je disais rien, je continuais à limer, je regardais la pendule mais je disais rien. Parce que le problème c'est qu'une fois, j'ai dit quelque chose et elle a dit que je lui ai répondu, puis elle a été dire à la maître [sic] d’apprentissage.

Cette stratégie semble directement liée au statut d'apprenti.e. Ayant difficilement la possibilité de faire entendre leurs voix, compte tenu de leur position hiérarchique, certain·e.s optent pour un silence qui semble plus «protecteur».

\section{La non-réaction}

L'effacement peut également prendrela forme dela non-réaction (Dejours, 2000b; Leclerc et Maranda, 2002). Il s'agit alors d'une attitude passive consistant à faire "profil bas", à ne pas réagir, à subir et à accepter les contraintes imposées. Dans un tiers des cas, les jeunes ne réagissent 
tout simplement pas à la souffrance qu'elles ou ils vivent $(\mathrm{N}=16)$. Ces personnes semblent ignorer la situation, comme le restitue Tiffany, une employée de commerce de 17 ans:

Je me suis complètement laissé marcher dessus [...]. Je me suis dit: «Ben, si ça passe pas, ça passera pas, quoi, c’est pas grave.»

\section{La soumission}

L'effacement peut encore prendre la forme de la soumission (Dejours, 1998; Maranda et Leclerc, 2000). Il s'agit là d'une forme de conformité, qui peut aller jusqu'à la servilité. Ce comportement est une acceptation consciente, une obéissance à l'autorité, à la hiérarchie, qu'elle soit fondée sur l'âge, l'expérience ou encore le sexe. Un quart des jeunes $(\mathrm{N}=12)$ accepte, à un moment donné du parcours en formation professionnelle duale, de faire preuve de soumission. Elles et ils développent ce type de comportement vis-à-vis de la hiérarchie et justifient leur souffrance comme si elle était normale et implicite à leur statut. Laetitia, une employée de commerce de 16 ans, en donne une parfaite illustration:

Moi, je voulais pas dire non, parce que je me disais: «Ouais, c'est mon patron! » [...]. Ils m’ont engagée, ils peuvent très bien me jeter! Donc je me suis dit: «Autant faire ces trucs-là. » [...]

J'étais apprentie, dans ma tête je me suis dit: «Ouais, je dis pas non à ce qu'ils me disent!»

\section{L'isolement}

L'effacement peut également passer par l'isolement (Leclerc et Maranda, 2002), qui est un comportement actif de repli sur soi, une auto-élimination, une auto-marginalisation. Pour affronter la souffrance ou la peur, la personne choisit de s'effacer de la scène. Un quart des apprenti.e.s réagissent à la souffrance en s'isolant $(\mathrm{N}=11)$. Elles et ils s'effacent de la réalité, rompent des liens pour s'en échapper. Ces jeunes adoptent une stratégie visant l'évitement "physique» du problème. C'est ce que fait Liam, un laborant en chimie de 17 ans:

Moi, au début, je m'entendais bien avec les gens là-bas et tout ça, et puis ben, après j'ai moins travaillé et tout [...] je commençais à moins parler avec eux et tout, petit à petit je commençais, pas à m'effacer, mais à parler de moins en moins 
[...]. Y a des journées, je ne sais pas, j’arrivais aux cours et puis je parlais quasiment pas.

Cette stratégie de retrait rappelle celle évoquée plus haut consistant à garder le silence. Dans les deux cas, les jeunes se retirent du milieu qui a engendré la souffrance, se mettent entre parenthèses. L'effacement est une stratégie qui peut être délétère, dans le sens où elle peut ne pas être comprise par l'environnement professionnel, que ce soient les collègues, les personnes formatrices ou encore les employeur.e.s. Comme certaines formes de dérobade, l'effacement peut être perçu comme un manque d'intérêt pour le métier, pour la formation professionnelle. Cette stratégie peut être interprétée comme un signe de démotivation.

\section{Les stratégies d'effacement, des stratégies épicènes?}

Les stratégies d'effacement sont autant utilisées par les apprenties que par les apprentis : la non-réaction (8 filles et 8 garçons), la soumission (6 et 6) et le silence (12 et 11). Elles ont en commun une certaine acceptation des contraintes imposées, une forme d'obéissance aux règles du monde du travail, règles fondées sur la hiérarchie (P. Kergoat, 2006), que celle-ci soit organisée autour de la question du statut, de l'âge, de l'expérience ou du sexe. Communes aux deux sexes, les stratégies d'effacement peuvent être qualifiées de stratégies typiques pour des jeunes qui viennent d'arriver sur le marché du travail et qui n'en connaissent pas encore les règles. Prudent.e.s, leur premier réflexe semble être de s'effacer, voire de se soumettre aux situations, même lorsqu'elles sortent de l'acceptable, afin d'être accepté.e dans l'entreprise. Sur ce plan, filles et garçons partagent la même condition, elles et ils occupent le bas de la hiérarchie au sein de l'entreprise et doivent s'en remettre à l'ensemble des collègues qui, par leur âge, leur expérience et leur position dans l'entreprise leur sont supérieur.e.s. En revanche, le statut des apprenti.e.s dans cette échelle hiérarchique ne prend pas le même sens pour les filles et les garçons: il correspond à celui des filles dans la hiérarchie entre les sexes, mais pas à celui des garçons, associés à la classe de sexe dominante. Pour eux, le statut d'apprenti les ramène à un statut de genre féminin, parce que les travaux d'entretien auxquels ils sont assignés au cours de leur formation professionnelle sont habituellement effectués, dans la sphère privée comme dans l'entreprise, par des femmes (voir Devreux, 1992). La formation professionnelle participe ainsi à l'apprentissage que filles et garçons font de la hiérarchie sociale, en particulier celle qui règne dans l'entreprise (P. Kergoat, 2006), et, partant, celle qui régit les rapports 
entre les sexes. Le recours à des stratégies d'effacement est donc sans doute pour les garçons une manière de protéger leur identité sexuelle menacée, même si ces stratégies leur permettent aussi, tout comme aux filles, de se faire reconnaître en tant que professionnels.

\subsubsection{L'accommodation}

Laccommodation regroupe des comportements par lesquels les jeunes cherchent à composer avecla situation, à tenir bon, notamment en recourant à des motivations extrinsèques. Par exemple, l'accommodation est soutenue par l'envie (et parfois la nécessité) d'obtenir un diplôme professionnel (CFC, Certificat fédéral de capacité). L’accommodation peut prendre les formes suivantes: l'endurance, le partage, l'hypercorrection et la virilité défensive. Dans le cas des apprenti.e.s en difficulté, opter pour des stratégies d'accommodation a pour but le maintien en formation et l'obtention de la qualification.

\section{L'endurance}

Près de deux tiers des jeunes du corpus cherche à s'accommoder, à composer avec la souffrance en mettant en avant la nécessité de tenir bon, ce qui a été appelé l'endurance (Leclerc et Maranda, 2002; Maranda et Leclerc, 2000). Il s'agit ici de tenir, de supporter, de résister dans la durée, de "faire le poing dans sa poche». De manière générale, il est difficile de savoir comment considérer l'endurance. En effet, d'une part, on pourrait la considérer comme une contrainte (et donc une source de souffrance), l'enjeu pour les jeunes étant de se maintenir en formation pour décrocher leur diplôme. D’autre part, elle figure dans nombre de témoignages comme le moyen de se maintenir en formation, elle pourrait donc être analysée comme une stratégie. Considérée comme une contrainte, l'endurance devrait être analysée plus finement: comment fait-on pour résister durablement, pour faire le poing dans sa poche, quelles ressources faut-il mobiliser? Il est difficile de trancher définitivement, aussi l'endurance est-elle, dans un premier temps, analysée comme une stratégie. Elle sera rediscutée plus bas dans ce chapitre.

Près de deux tiers des jeunes $(\mathrm{N}=27)$ adoptent cette stratégie, dont les manifestations peuvent être multiples. Signalons également que la durée durant laquelle les personnes y recourent varie d'une situation à 
l'autre. La gardienne d'animaux mentionnée ci-dessus a, par exemple, démontré une endurance exceptionnelle ${ }^{91}$ :

J'ai tenu le coup pendant une année et demie mais ça a été tout du long depuis ce 12 juillet 2004 [date d'entrée dans l'entreprise], c'était le harcèlement moral complet!

Il est aussi à constater que les jeunes qui optent pour l'endurance expliquent souvent l'utilisation de cette stratégie par des arguments liés à leur motivation ou à la nécessité de mener à terme une formation post-obligatoire. Stéphanie, une employée de commerce de 19 ans, l'exprime très clairement:

Je me forçais à y aller [au travail] parce que je m'étais fixée le but de le [le certificat de formation professionnelle] réussir.

L'importance d'obtenir le CFC revient fréquemment dans les propos des jeunes comme argument justifiant qu'elles et ils tiennent bon le plus longtemps possible. Cela met en exergue l'importance de ce diplôme pour cette population et plus généralement pour les personnes qui les entourent (famille, corps enseignant, collègues). Finalement, l'endurance n'est qu'une stratégie passagère, en tout cas dans nos témoignages. En effet, les apprenti.e.s tiennent bon jusqu'à ce que la situation ne soit plus gérable. Arrivéee-s au bout de leurs capacités d'endurance, ces jeunes changent de stratégie ou rompent leur contrat, comme la gardienne d'animaux précédemment citée:

Je savais qu'on me dansait sur le ventre et tout, [...] il y avait vraiment des trucs [...] qui étaient lourds à supporter... je rentrais tous les jours chez moi en pleurs! C'était limite dépression! Mais j'essayais de tenir le coup en me disant que c'est pour mon apprentissage que je le fais, que c'est important. Et pour finir j'ai craqué et ça allait vraiment plus!

Ce dernier extrait met en évidence le statut ambigu de l'endurance. Il s'agit d'une stratégie, l'intention est bien ici de «tenir le coup», mais elle est parallèlement une contrainte (tenir pour la réussite del'apprentissage), qui génère de la souffrance. Au terme de son expérience, Aurélie semble avoir souffert autant à cause des problèmes rencontrés dans l'entreprise,

91 Préalablement à la signature de son contrat d'apprentissage, Aurélie a effectué un stage d'une année dans la même entreprise. Cela explique la durée durant laquelle elle a fait preuve d'endurance, et ce bien qu'elle ne soit formellement qu'en première année. 
des mauvaises relations de travail, que de cette quasi-obligation à tenir bon, à résister jusqu'à l'obtention du diplôme.

\section{Le partage}

Plus d'un tiers des jeunes, en grande majorité des filles, optent pour cette forme de stratégie d'accommodation $(\mathrm{N}=19)$. Il s'agit d'une stratégie inédite. C'est l'antithèse du silence et de l'isolement, sans pour autant être un processus de dénonciation. Si elle s'apparente à la prise de parole (Maranda et Leclerc, 2000), cette stratégie consiste surtout à faire largement part de ce qui arrive. Le but est non seulement de se défouler, mais également d'être entouré.e, soutenu·e, conseillé.e, afin de se faire une opinion et de prendre une décision. C'est en l'occurrence aux proches (parents, collègues, pairs) que les jeunes parlent des problèmes rencontrés durant leur formation, soulignant par-là l'importance du réseau comme ressource. Le recours aux proches permet d'obtenir conseils, soutien affectif et aide pratique, c'est ce qu'attendent par exemple Bastien, un monteur-électricien de 15 ans ( ${ }^{\text {er }}$ extrait), et Célia, une coiffeuse de 18 ans ( $2^{\mathrm{e}}$ extrait):

Si j'ai des problèmes [...], je sais que je peux causer à mes collègues, parce que ça ils me le disent tous les jours [...], puis ben, on en cause souvent...

Je demande aussi beaucoup conseils, c'est vrai que j'ai besoin d'être soutenue, d'être rassurée, d'avoir del'aide, c'est vrai que j'en parle beaucoup, à mes copines surtout...

\section{L'hypercorrection}

L'accommodation peut prendre la forme de l'hypercorrection (Leclerc et Maranda, 2002). Il s'agit alors d'une intériorisation et d'une reprise à son compte des contraintes subies. Les personnes utilisant ce type de stratégie ont fréquemment des pratiques d'excès (heures supplémentaires, nuits blanches) et se conforment, parfois de façon extrême aux attentes et aux normes du cadre de travail. Plus d'un quart des jeunes, dont une nette majorité de filles, réagissent à leur situation de souffrance en s'engageant de manière disproportionnée dans leur formation professionnelle $(\mathrm{N}=13)$. L'hypercorrection est une manière d'aller au-delà des attentes explicites de l'institution pour tenter d'éviter de souffrir ou pour répondre à la peur de ne pas être à la hauteur (voir Chapitre 6). Pour y parvenir, les personnes se distinguent par exemple en s'investissant à fond dans leur 
travail, en se montrant particulièrement performantes ou en faisant des heures supplémentaires. Deux employées de commerce, Tiffany 17 ans ( $1^{\text {er }}$ extrait) et Stéphanie 19 ans ( $2^{\mathrm{e}}$ extrait) montrent jusqu'où peut aller cet engagement :

Moi, je suis toujours à jour, j'ai jamais rien à faire. Parce que, je veux dire: je bosse rapidement et je contrôle tout le temps, cinq à six fois. Donc il y a toujours des collègues qui nagent, ou comme ça, alors à chaque fois je fais «est-ce que je peux te faire quelque chose», ou comme ça.

Un bon apprenti, c'est [celui] qui fait ce qu'on lui demande, plus encore un petit peu plus [...]. Dans tous mes stages, j'essaie de faire plus qu'on me demande, si je peux.

\section{La virilité défensive}

Laccommodation peut enfin prendre, dans certains cas, la forme de la virilité défensive (Dejours, 1998, 2000b; Molinier, 2002a; Molinier et Dejours, 1997). Chez les cadres, les ressorts de la virilité sont utilisés pour leur faire exécuter des tâches que leur sens moral les retiendrait de faire (comme faire souffrir, licencier). Toute résistance à l'exécution de ces tâches est qualifiée de non-virile. Mais la virilité au travail se retrouve également sous une autre forme, celle des «idéologies défensives de métier» (Dejours, 2000a, 2000b). Il s'agit pour des travailleurs, tels que les ouvriers du bâtiment, les opérateurs du nucléaire, les personnes travaillant dans l'industrie chimique, de construire autour des valeurs de virilité, tout une série de comportements, d'interdits, de non-dits une idéologie centrée sur le courage et la force. Cette idéologie défensive, s'apparente au déni et met en avant la bravoure, la prise inconsidérée de risques, le défi ${ }^{92}$. Le recours à la virilité défensive apparaît très rarement dans le corpus, et uniquement chez des garçons $(\mathrm{N}=3)$. Ceux-ci sont

92 Bien que Dejours distingue le cynisme viril des cadres (Dejours, 1998, 2000b) de l'idéologie défensive des travailleurs actifs dans des tâches connotées comme masculines (Dejours, 2000a), j'utiliserai ici le terme de virilité défensive de manière indifférenciée. En effet, si l'on considère les métiers concernés (formation professionnelle) ou les statuts occupés (apprenti.e.s), le corpus devrait être analysé sous l'angle des idéologies défensives. Pourtant, le fait d'avoir affaire à des jeunes en tout début de formation exclut leur participation à l'élaboration collective d'une telle idéologie. Ce que je souhaite avant tout mettre en évidence ici est la construction de systèmes défensifs fondés sur une représentation magnifiée de la virilité. Ces systèmes se construisent autour de normes de virilité érigées en modèles et 
insérés dans les formations du secteur du bâtiment et de l'industrie automobile, c'est-à-dire des métiers très masculins, où l'on retrouve la composante virile dans les idéologies défensives mises en œuvre de façon relativement courante par leurs aînés. Il s'agit en effet de dépasser la peur engendrée par l'activité ou l'environnement dans lequel elle s'exerce. C'est le cas de Jacques, le menuisier de 18 ans déjà évoqué précédemment pour la phobie des machines qu'il a développée suite à des accidents :

Quand je voyais que j'arrivais plus à travailler, je me suis dit: «Faut que j'arrête!» Mais je préférais continuer, essayer de, de com... de battre ma peur.

Combattre sa peur, c'est affronter le problème, c'est refuser l'évidence de l'impossibilité de poursuivre sa formation, alors qu'il y a un risque si ce n'est pour la santé physique (les accidents n'étaient pas trop graves), du moins pour la santé mentale. Cette stratégie virile se retrouve également dans le refus de partager sa souffrance, que ce soit avec ses ami.e.s ou avec les chercheur.e.s (voir Chapitre 6). Dire sa souffrance, avouer sa «faiblesse» serait une atteinte à la virilité. C'est ce qu'exprime Robert, un charpentier de 16 ans:

J'ai trop parlé à mes copains. [...] Pour moi, c'est pas utile de parler, quoi.

[...] J’aime bien gérer tout seul quoi, c'est mon problème.

Le fait que la virilité défensive soit peu utilisée suggère qu'il s'agit d'une stratégie d'adulte. En outre, il s'agit d'une réponse collective, voire dans certaines situations d'une idéologie défensive de métier, qui s'acquiert durant la socialisation professionnelle dans un collectif de travail.

\section{Des stratégies d'accommodation marquées du sceau du genre} Hormis une stratégie faisant partie de ce groupe et qui est utilisée par les jeunes des deux sexes, les stratégies d'accommodation sont assez nettement sexuées. Ainsi, à part l'endurance utilisée par filles et garçons (15 pour 12), le partage et l'hypercorrection sont largement utilisée par les jeunes femmes ( 14 pour 5 pour le partage, 10 pour 3 pour l'hypercorrection), pendant que seuls les garçons recourent à la virilité défensive.

L'endurance est l'une des stratégies les plus utilisées par les jeunes, encore plus par les filles; elle s'inscrit dans la suite logique des stratégies

fonctionnent sur le rejet, le mépris de tout ce qui en diffère, en particulier le féminin et ce qui y est associé. 
d'effacement vues précédemment. L'écart entre les sexes peut s'expliquer par la construction sociale de filles «scolaires » et de garçons turbulents, qui s'est faite tout au long de la scolarité, en amont de la formation professionnelle (Dafflon-Novelle, 2006; Duru-Bellat, 1990 ; Mosconi, 1994). Cette stratégie s'inscrirait ainsi dans une logique similaire aux stratégies de dérobade, reproduisant des rapports à la formation professionnelle sur le modèle de ceux ayant été construits précédemment avec l'école.

En ce qui concerne le partage, stratégie typique d'une population adolescente, elle apparaît plus chez les filles. En effet, ces dernières ayant été socialisées à développer leurs compétences sociales, à parler de leur état émotionnel (Dafflon-Novelle, 2006), le partage semble une stratégie conforme à leur genre. Ce phénomène se retrouve dans certaines recherches sur les relations des adolescent.e.s avec les intervenant.e.s sociales et sociaux, où il apparaît que les filles mettent davantage en évidence le «besoin de parler», de partager (Coutant, 2007).

De manière globale, les stratégies d'accommodation visant le maintien en formation et l'insertion durable dans un collectif de travail, il n'est guère surprenant de voir apparaître une nette séparation entre filles et garçons. En effet, dans ce type de stratégie, il est indispensable de s'adapter à un contexte professionnel sexué. Hypercorrection et partage pour des jeunes femmes dans des milieux de travail largement féminisés et virilité défensive pour des jeunes hommes devant faire leur preuve dans des univers professionnels à dominance masculine. Les stratégies d'hypercorrection et de virilité défensive qui se construisent sur l'adoption de comportements identifiés socialement comme étant typiquement féminins ou masculins seront analysées plus bas comme stratégies de genre.

\subsubsection{L'affirmation}

L'affirmation est une stratégie visant le changement et orientées vers le problème et sa résolution. Elle peut prendre différentes formes, comme l'action, la réaction et la concurrence. Ces stratégies semblent se situer au-delà d'une simple défense de l'intégrité psychique ${ }^{93}$. On est ici déjà

93 Dejours (2000b) rappelle que les stratégies de défense sont légitimes, car elles permettent à l'individu de protéger sa santé mentale. Ce faisant, elles empêchent parfois le recours à l'action individuelle et collective en faveur de l'amélioration du rapport entre l'individu et le travail, ou si l'on se place dans une perspective syndicale ou ergonomique, en faveur de l'amélioration des conditions de travail. 
dans la mise en œuvre d'éléments destinés à modifier l'environnement de travail, à en faire changer les conditions.

\section{L'action}

Plus des deux tiers $(\mathrm{N}=34)$ des apprenti.e.s adoptent l'action pour s'affirmer, défense qui n’a pas été retrouvée dans la littérature. Sous ce terme, est regroupée toute tentative active de résolution de la situation de souffrance. Cette stratégie se rapproche, tout en s'en différenciant, de la stratégie de réaction. En effet, si elles ont en commun le refus du statu $q u o$, l'action concerne des démarches qui se centrent sur le problème et sa résolution, alors que la réaction consiste, comme on le verra plus loin, à dénoncer ou à se révolter contre la situation. Les stratégies d'action directe comportent des démarches concrètes que les jeunes proposent et mettent en œuvre pour résoudre leurs problèmes, comme décider d'arrêter la formation, consulter l'office d'orientation professionnelle, appeler la ou le commissaire d'apprentissage ou tenter une discussion avec la hiérarchie. Anaëlle, une horticultrice de 18 ans, a mis différentes choses en œuvre:

Moi, ce que j'avais proposé c'est [...] déjà d'expliquer ce qui allait pas, et puis de refaire un test pendant un mois, puis voir si les choses s'arrangeaient ou pas. [...]

Sinon, j'ai passé aussi à l'orientation professionnelle, ici à Lausanne.

L'action indirecte implique la sollicitation de soutiens externes. En particulier lors de situations relationnelles difficiles, les jeunes demandent à une tierce personne, souvent membre de leur famille, d'intervenir dans la résolution du conflit. C'est le cas d'Aurélie, une gardienne d'animaux de 17 ans, qui demande l'aide de sa mère:

J'arrive pas à me défendre, encore, parce que j'ai pas assez de force, justement, pour me défendre. Alors, ben, j’allais vers ma mère, parce qu'en plus j'étais, je suis toujours mineure, alors j'allais vers elle et c'est elle qui me protégeait, c'est elle qui intervenait.

\section{La réaction}

L'affirmation se manifeste aussi parfois sous la forme de la réaction (Leclerc et Maranda, 2002). Contrairement à l'action vue ci-dessus, il 
s'agit ici d'une non-acceptation active et démonstrative. La personne optant pour cette forme d'affirmation dit son refus, se confronte, conteste. Sa résistance peut prendre la forme d'une certaine indiscipline. Plus du tiers des jeunes du corpus recourent à la réaction $(\mathrm{N}=18)$. Ses manifestations sont très variées, mais dans la majorité des cas, elles se limitent à des réactions de type verbal. Stéphanie, une employée de commerce de 19 ans ( $1^{\text {er }}$ extrait), et Matthieu, un vendeur de 16 ans ( $2^{\mathrm{e}}$ extrait), en donnent une bonne illustration:

Je lui ai dit que maintenant ça allait suffire, que j'étais pas handicapée, que je faisais mon travail comme tout le monde.

Moi, j’ai pas trop apprécié, je lui ai dit: «Vous auriez pu me dire, justement, qu'est-ce qui vous plaisait pas en moi, puis j'aurais pu essayer d'améliorer, puis comme ça moi, je vous aurais dit aussi qu'est-ce qui [...] allait pas.»

Certain.e.s jeunes réagissent par d'autres biais, par exemple par une prise de conscience ou par des actes (et non des mots) de "révolte». C'est le cas de Agusto, un dessinateur en bâtiment de 18 ans:

Ils ne voulaient pas me donner toutes mes semaines de vacances qui me restaient, $[\ldots]$ mais je les ai quand même prises, puis il a quand même été d'accord.

\section{La concurrence déloyale}

L'affirmation peut prendre la forme d'une concurrence déloyale (Dejours, 1998, 2000b). Ici la personne qui souffre adopte des conduites déloyales et se met en compétition effrénée avec ses collègues, dans le but de «sauver sa peau ». C'est la forme de l'affirmation la moins utilisée par les jeunes $(\mathrm{N}=2)$, probablement parce que la plupart ne sont pas en position (statutaire, hiérarchique) pour le faire. Pourtant, une apprentie se met volontairement en concurrence avec une de ses collègues, qui lui mène la vie dure, et un apprenti dénonce les agissements de ses collègues à son patron. Ce faisant, ces jeunes entrent en concurrence avec des collègues qui les ont fait souffrir et cherchent à les discréditer. Tiffany, une employée de commerce de 17 ans, compromet ainsi sa collègue:

Je fais [à la collègue] : «Attends, je vais vers le patron voir s'il est pareil que toi.» Je suis partie vers le patron, je fais: «Oui, c'est une personne [un locataire], y a son appartement qui a 
pris feu, parce que celui d'au-dessous a pris feu aussi.»Pis, il [le patron] fait: "Ah passez-moi, y a pas de problème.» Et le patron a déjà vu ça: magnifique! Et l'autre [sa collègue], quand je suis revenue dans le bureau, elle était rouge, pis elle me dit: «T’as pas à prendre mes lignes [téléphoniques] !» Pis je fais: «Excuse-moi, mais ton appart', il prend feu, on t'envoie chier, tu fais quoi?»

Dans ce cas-là, l'apprentie estime faire son travail, elle considère qu'on ne peut pas traiter la clientèle comme l'a fait sa collègue (souffrance éthique), et en profite pour discréditer celle-ci aux yeux du patron et du locataire.

\section{L'affirmation une stratégie teintée par le genre}

Comme la dérobade et l'accommodation, l'affirmation est utilisée par les jeunes des deux sexes, mais avec une utilisation spécifique des différentes stratégies qui composent ce groupe. Ainsi, la concurrence est-elle utilisée de façon paritaire, mais très rarement (1 pour 1$)$, l'action est privilégiée par les jeunes femmes (20 pour 14), pendant quela réaction est davantage utilisée par les jeunes hommes (10 pour 8).

Ici encore, les écarts sont très faibles et on peut probablement expliqués ces choix distincts par l'insertion professionnelle dans des secteurs sexués, où les normes diffèrent fortement. On peut supposer, qu'il est davantage attendu des garçons qu'ils fassent preuve de virilité. Pour éviter d'adopter des comportements qui seraient considérés comme lâches, ils chercheraient alors à régler la situation, quitte à aller à la confrontation, brefà être réactifs. Parallèlement, il est socialement ad mis pour les filles d'éviter les situations de confrontation, de demander de l'aide à autrui, mais surtout il est attendu d'elles qu'elles recourent à leurs compétences sociales, de dialogue et ainsi qu'elles mettent en place des actions pour sortir de la situation. Ainsi, derrière d'apparentes similarités de stratégies adoptées, se rejouent les normes sociales en vigueur pour chacun des groupes de sexe.

\subsection{Des stratégies révélatrices du statut d'apprenti·e}

La population apprentie n'a jamais fait l'objet d'une analyse en termes de stratégies défensives. Ainsi, certaines stratégies inédites, l'action et le partage, sont apparues grâce à la prodécure inductive. Par ailleurs, toujours en lien avec cette population, son statut particulier et sa position dansl'organisation du travail, certaines stratégies répertoriées dans la littérature n'ont que peu été retenues, certaines n'ont même pas été 
utilisées. Ensuite, une stratégie, l'endurance, a été massivement retenue par les jeunes, ce qui renvoie également à leur situation spécifique. Enfin, certaines stratégies seront rediscutées à l'aune du genre. Certaines sont en effet utilisées en priorité par les hommes (virilité défensive), par les femmes (hypercorrection) ou illustrent un type d'insertion particulier, celui des personnes pionnières.

\subsubsection{Des stratégies inédites}

L'analyse des entretiens révèle qu'une partie non négligeable des jeunes en formation professionnelle duale utilise deux stratégies inédites: l'action et le partage. La présence de ces deux nouvelles stratégies met en évidence la spécificité du phénomène analysé ici, tant au niveau du champ (la formation professionnelle) que des caractéristiques de la population étudiée (l'adolescence).

L'action peut êtreliée à la spécificité du champétudié. L'entrée en formation professionnelle correspond en effet aux premiers «tâtonnements » des apprenti.e.s dans le monde du travail. Ne sachant pas comment réagir aux contraintes qu'elles et ils subissent, les apprenti.e.s essaient d'agir, avec les moyens à leur disposition. Elles et ils activent leur réseau familial, amical, institutionnel. Relevons ici, que comparé.e.s aux travailleuses et travailleurs adultes, les jeunes en formation professionnelle duale ont la possibilité de solliciter davantage d'appuis institutionnels. La formatrice ou le formateur en entreprise, la ou le commissaire d'apprentissage (censé.e intervenir auprès de l'entreprise et des jeunes en cas de litige), l'office d'orientation, les conseillères ou conseillers aux apprenti.e.s, le corps enseignant, le service de médiation des écoles professionnelles sont autant d'instances ou de personnes mobilisables en cas de difficultés. Malgré cet important réseau, les ruptures ne peuvent pas toujours être évitées, car les jeunes n'ont pas nécessairement recours à ces ressources et ne sont pas toujours informé.e.s de leur existence. Enfin même si ces appuis sont sollicités à temps, le problème n'est pas résolu pour autant.

Quant au déploiement relativement fréquent de la stratégie de partage, son caractère inédit peut s'expliquer par le fait que la population adolescente n'a pas été approchée par la psychodynamique du travail. Le partage serait une stratégie spécifique et typique de l'adolescence, moment de constitution des identités personnelle et sociale. Les jeunes sont portés à intensifier et à réorganiser leurs relations sociales, à tester et construire de nouvelles modalités d'interaction, à s'intégrer à de nouveaux groupes sociaux, ainsi qu'à se repositionner dans leur environnement social (famille, école, pairs, etc.). De plus, la construction identitaire à 
cette période de vie est tributaire du regard de l'autre, l'adolescent.e se définissant à travers l'image que lui restitue son entourage (Moulin, 2005). Ainsi, l'importance de l'aspect relationnel - aussi bien pour se positionner dans l'environnement que pour se définir à travers le regard de l'autre - porte les jeunes à échanger spontanément et fréquemment avec autrui et à soumettre à autrui leurs propres doutes, angoisses, peurs et souffrances.

\subsubsection{Des stratégies délaissées}

Certaines stratégies de défense observées dans cette recherche ne reproduisent que partiellement celles mises en évidence par la psychodynamique du travail: la concurrence déloyale et la virilité défensive ne sont pratiquement pas adoptées par les apprenti.e.s interviewé.e.s, alors que le clivage, l'individualisme, la collaboration et la muliérité ${ }^{94}$ ne se retrouvent pas du tout dans le corpus. Elles ne sont vraisemblablement pas activées par la population spécifique des jeunes en début de formation professionnelle duale. Certaines d'entre elles nécessitent une position particulière dans l'entreprise ou un autre statut que celui d'apprenti.e.

On peut se demander pourquoi les jeunes en situation de souffrance professionnelle n'y recourent que très rarement. A nouveau, ceci pourrait être dû au fait qu'il s'agit de leurs premiers contacts avec le monde du travail, elles et ils n'auraient pas encore intégré toutes les normes, les valeurs et les codes de conduite du monde du travail (Dubar, 1996). Ce manque d'expériences professionnelles fait que les jeunes n'ont pas encore eu le temps de s'approprier des stratégies que les travailleuses et travailleurs adultes mobilisent. Comme déjà évoqué, certaines stratégies «s'apprennent» au cours de la socialisation professionnelle et font véritablement partie de l'identité professionnelle de certains métiers. Elles ne sont dès lors pas encore accessibles à ces jeunes travailleuses et travailleurs.

Les moyens non retenus témoignent également du statut particulier des apprenti.e.s. Leur position hiérarchique les exclut de fait de certaines stratégies. En effet, pour collaborer à ce qu'on réprouve, c'est-à-dire pour participer au système qui engendre de la souffrance, pour reproduire sur autrui ce que l'on subit (Dejours, 1998), il faut être en mesure de le faire, c'est-à-dire avoir une parcelle de pouvoir. Or, la personne hié-

94 La muliérité est la seule stratégie à avoir été ajoutée au moment de l'analyse secondaire. Elle n'apparaît pas comme telle, car dans la suite de l'analyse, l'hypercorrection sera réenvisagée en partie comme une manifestation de la muliérité. 
rarchiquement la plus faible n'en a objectivement pas les moyens. Par ailleurs, le clivage, soit le cloisonnement des sphères de vie qui permet de séparer celle où l'on inflige de la souffrance à autrui (le travail) de celle qui n'entre pas en contact avec cette souffrance (la sphère privée) (Dejours, 1998, 2000b), n'est nécessaire qu'en parallèle à une stratégie de collaboration, et ce afin de se protéger. Au vu de ce qui précède, il n'a donc aucun sens ici. Enfin, l'individualisme, c'est-à-dire la politique $\mathrm{du}$ «chacun pour soi» où l'on défend ses propres intérêts (Dejours, 2000b; Leclerc et Maranda, 2002) ne peut pas être choisi, puisqu'étant en formation, l'apprenti.e a besoin de ses collègues, accessoirement de ses formateurs et formatrices.

\subsubsection{Des jeunes qui font le poing dans leur poche}

L'endurance qui apparaît massivement dans les témoignages semble indiquer que les personnes interrogées sont conscientes de l'importance de leur formation et des dangers d'une interruption. En effet, dans le cas contraire, les jeunes ne s'imposeraient pas la souffrance engendrée par la situation. En ce sens, et même si cet aspect n'a pas été analysé dans le présent chapitre, remarquons que les témoignages révèlent une extrême lucidité des jeunes interviewé.e.s quant au rôle, aux enjeux de la formation professionnelle, ainsi qu'à l'importance du diplôme. Elles et ils apparaissent - dans cette étude, comme dans une recherche longitudinale menée auprès de trois mille jeunes en Suisse (COCON, 2006) - comme des adolescent.e.s motivé.e.s et disposé.e.s à s'investir pour pouvoir poursuivre et menerà terme une formation post-obligatoire.

L'importance accordée à la formation, et au diplôme qui la sanctionne, fait de l'endurance un véritable enjeu. Il s'agit de tenir jusqu'au bout, de faire le poing dans sa poche durant les trois ou quatre années que dure la formation professionnelle duale. C'est dans ces termes, que certain.e.s apprenti.e.s ont évoqué leur expérience. Dès lors, on peut effectivement considérer l'endurance comme une contrainte. Il s'agirait ainsi d'affiner l'analyse et de s'interroger sur les diverses stratégies mises en œuvre pour tenir bon, pour faire le poing dans la poche. Une relecture dans ce sens des diverses stratégies évoquées précédemment serait intéressante.

Après ces premières réflexions, il semble qu'une clef d'analyse possible des stratégies face à la souffrance au travail chezles jeunes pourrait être la temporalité. En effet, l'une des stratégies les plus largement adoptées par les apprenti.e.s (l'endurance) est une stratégie de la durée. Et ce n'est qu'au terme d'un temps certain que cette stratégie est doublée, 
voire remplacée par une autre. Pourtant, la plupart des autres stratégies semblent elles aussi être des réponses ponctuelles. Leur relecture sous l'angle de la temporalité est d'autant plus intéressante qu'il s'agit d'une population adolescente. L'ancrage des adolescent.e.s dans le présent ou dans un futur proche (Boivin et Fournier, 2006; Trottier, 2006) et leur statut provisoire impliquent en effet que leurs stratégies sont souvent des réponses immédiates. La question semble être, dès lors, «que puis-je faire toute de suite pour me protéger?", d'où le recours à la non-réaction, au silence, à la soumission, voire à l'hypercorrection. Ces stratégies illustrent l'analyse que font les jeunes de la situation: elles et ils la perçoivent comme transitoire, espérant qu'elle passe. D'autres stratégies semblent par contre s'ancrer davantage dans une temporalité du futur proximal. Dans ce cas, les jeunes parviennent à se projeter dans un avenir proche, où la formation sera achevée et le diplôme obtenu. C'est cette projection à moyen terme qui leur permet d'opter pour des stratégies de longue haleine, telles que l'endurance.

\subsubsection{Des stratégies de genre}

Commeévoqué précédemment, les jeunes recourent principalementà des stratégies de défense individuelles, les stratégies collectives de défense ou les idéologies défensives étant rares. Ces stratégies individuelles renvoient aux autres aspects de leur socialisation, de genre en particulier. Des contextes professionnels différents générant des souffrances particulières (voir Chapitre 6), il est donc probable que les stratégies varient, elles aussi, selon les environnements dans lesquels elles sont produites. Ainsi, le système de genre, qui structure les différentes réalités professionnelles, agirait sur les stratégies retenues. Tout d’abord, les stratégies peuvent différer selon les contextes professionnels (ségrégués) dans lesquels elles sont utilisées. Ensuite, elles s'inscrivent en prolongement d'une socialisation de genre, les personnes adoptant des comportements socialement admis (pour leur sexe, mais aussi pour leur milieu social par exemple). Enfin, elles sont probablement instrumentalisées par le système de genre, faire le choix d'une stratégie permettrait d'asseoir son identité de genre.

Trois stratégies doivent être analysées séparément, car elles sont employées de manière prioritaire, si ce n'est exclusive, par l'un ou l'autre sexe. Il s'agit du déni (5 garçons pour 2 filles), de l'hypercorrection (10 filles pour 3 garçons) et de la virilité défensive (3 garçons). Cette analyse regroupera le déni et la virilité défensive comme stratégies de genre plus spécifiques aux métiers masculins et aux garçons, et considérera 
l'hypercorrection comme une stratégie de genre caractérisant avant tout les métiers féminins et les filles.

Le déni peut en effet être analysé comme faisant partie de la virilité défensive, car celle-ci repose notamment sur un déni de perception (Dejours, 1998, 2000b; Molinier, 2000). Cette négation de la réalité difficile apparait bien chez Hakem, un réparateur automobile de 19 ans :

En fait, moi je suis quelqu'un qui oublie pas facilement, mais jusqu'au bout j'essaie d'oublier. Si j'arrive pas, ça reste dans la tête, mais je veux le laisser de côté et penser à autre chose.

Avant d'analyser plus en détail les deux stratégies de genre que sont la virilité défensive (dans laquelle est inclu le déni) et l'hypercorrection, rappelons que ce sont là deux stratégies d'accommodation, et qu'en tant que telles, elles indiquent la manière dont les jeunes cherchent à se maintenir en formation professionnelle. Ici, il s'agit de voir comment cette accommodation se fait selon le genre, ou, en d'autres termes, comment les apprenti.e.s composent avec les normes de genre en vigueur dans l'univers professionnel. La division sexuelle du travail et la position des jeunes travailleuses et travailleurs dans les rapports sociaux de sexe font que pour se maintenir dans le champ professionnel, il faut comprendre et endosser ces normes de genre.

\section{Faire fi de la peur et de la souffrance: la virilité défensive}

Cette stratégie joue sur les valeurs de la virilité et met en avant la bravoure, la prise inconsidérée de risques, le défi. A ces comportements s'ajoutent des interdits absolus de toute allusion à la peur ou à la souffrance.

Les jeunes hommes du corpus qui recourent aux stratégies de virilité défensive (y compris au déni), sont insérés dans des secteurs traditionnellement masculins: le bâtiment (menuisier, charpentier), l'industrie (laborantin en chimie, monteur-électricien et informaticien), l'industrie automobile (peintre en carrosserie, réparateur automobile), ainsi qu'un secteur plus mixte, la restauration et l'hôtellerie (pâtissier-confiseur) ${ }^{95}$.

Dans le chapitre précédent, la difficulté des jeunes hommes à manifester leurs peurs et leur souffrance faisait déjà état, en soi, d'une forme de virilité défensive. Ici, à travers les quelques témoignages des garçons qui s'essaient à ce type de stratégies, il s'agit de comprendre

95 La profession est déjà fortement féminisée, puisque près de $40 \%$ de jeunes femmes la composent: http://www.vd.ch/fr/themes/formation/orientation/ professions/zoom-sur-les-metiers/liste-des-emissions/patissier-confiseur-patissiere-confiseuse/ 
comment, au sein de l'entreprise, ils sont socialisés à la virilité. Certes leur socialisation professionnelle n'en est qu'à ses débuts, mais les récits de ces quelques garçons laissent entrevoir que les stratégies de virilité défensive déploieront tous leurs effets à partir du moment où les apprentis sortiront de leur statut d'individus en formation et s'intégreront dans un collectif de travail.

Dans un premier temps, on constate que les tentatives de combattre sa peur existent chez ces garçons, mais qu'elles sont mises en œuvre sans succès, comme on l'a vu avec Jacques, le menuisier de 18 ans déjà évoqué précédemment:

Je préférais continuer, continuer, voir, essayer de com..., de battre ma peur, mais j'arrivais pas.

La volonté de surmonter la peur qu'il ressent, lui est «enseignée» par ses collègues et par son formateur en entreprise. Le jeune homme est encouragé à tenir bon, à dominer ses craintes:

J'ai dit au patron que j'avais peur des machines tout ça, pis il a un peu tiré la gueule... pis, il m’a dit: «Essaie de com..., essaie de battre ta peur.»

L'apprentissage du déni de la peur est le propre des stratégies de défense viriles. La montrer est difficile, quasi impossible, car cela rappelle aux autres que le danger existe, que les accidents avec les machines ont lieu, qu'il y a des risques. La première réaction de l'employeur l'atteste («il a un peu tiré la gueule»). Or, ce "processus fragile nécessite l'adhésion de tous, et cela partout où la peur et la vulnérabilité pourraient apparaître » (Molinier, 2000: 29).

L'intérêt de ces récits est moins de voir ces stratégies en œuvre que de comprendre comment elles sont inculquées, enseignées. Ainsi, lorsque les encouragements ne fonctionnent pas, les jeunes hommes expérimentent à leurs dépens le machisme, la violence entre hommes (Welzer-Lang, 2000). Cette expérience leur signale quelle est la norme dominante et quel en est le repoussoir; le tout construit sur une grille de virilité/féminité (Molinier, 2000). Alessandro, un peintre en carrosserie de 19 ans, en fait l'expérience:

Avant c'était directement contre moi, mais là, ça allait, parce que j'ai jamais pris de coup au moral, [...] j'me suis toujours relevé. Mais là, cette fois quand il [son formateur] est allé plus loin, j’ai pas aimé et pis ça fout un coup quand même 
quand on va vers son apprenti et on dit: «Ton père, il a pas de couilles!», sans raison.

Dans cet extrait, le jeune homme montre à la fois qu'il sait faire face, qu'il ne se laisse pas affecter, qu'il se relève de la «violence ordinaire », et qu'il fait donc montre d'une certaine bravoure. Cependant, il est touché par les attaques où l'on remet en cause la virilité de son père. L'agression est particulièrement brutale, car on lui signifie que son manque de virilité remet en cause celle de son père, bref qu'il castre symboliquement son père, qu'il est responsable de sa déchéance. S’il peut ne pas répondre aux agressions quotidiennes dont il fait l'objet, il est par contre tenu de réagir dès lors que l'attaque est dirigée contre son père. La virilité se joue ici à défendre l'honneur familial. Parallèlement, cette attaque lui signale que l'ordre social repose sur la norme de la virilité, qu'on l'en exclut et que cette exclusion affecte toute sa famille.

\section{Se conformer aux attentes supposées du travail: I'hypercorrection}

Cette stratégie est une forme d'hyper-adaptation au monde du travail, une « sursocialisation » où les personnes se conforment aux normes supposées, et non nécessairement réelles, d’une activité professionnelle. Selon Eckert et Sulzer (2007), tandis que les jeunes hommes usent de virilisme face aux contraintes organisationnelles, les jeunes femmes font preuve d'une plus grande docilité. On peut dès lors se demander dans quelle mesure cette docilité se retrouve dans ce qui est appelé ici l'hypercorrection.

Dans un premier temps, il apparaît que cette stratégie se manifeste souvent au niveau relationnel. Les personnes qui l'emploient, des filles surtout, adoptent des comportements de sur-adaptation à leurs collègues, y compris lorsque ces personnes les maltraitent. C'est le cas d'Aurélie, une gardienne d'animaux de 17 ans, victime de mobbing et qui pourtant opte pour ce mode de comportement:

J'essayais tout le temps de... d'être sympa avec eux! Donc quand je savais que c'était son anniversaire, ben je lui avais amené quelque chose... Quand c'était les jours comme Noël ou Halloween ou comme ça, je leur ramenais à tous un petit paquet de bonbons ou comme ça...

Dans un certain nombre de témoignages, cette hypercorrection prend donc la forme «d'être gentille», que ce soit comme ici en offrant de petits 
cadeaux ou en prenant en charge le travail de collègues, allant parfois jusqu'à la servilité. Cette situation extrême est très bien illustrée par Leonora, une assistante dentaire de 16 ans :

J'avais peur, d'un côté que ça vienne de moi, que c'était à cause de moi, mais au bout d'un moment j'ai remarqué que ça venait pas de moi [...] J'faisais plein d'efforts, quand il fallait nettoyer quelque chose et qu'Amélie [prénom fictif] devait le faire, j'lui disais: «Si tu veux, j'peux le faire» ou si elle faisait tomber quelque chose, j'me baissais pour elle. Même si elle me disait pas de le faire, je le faisais quand même pour être sympa et tout.»

Cet extrait montre à la fois les formes de soumission que peut prendre l'hypercorrection, et l'insécurité commel'incompréhension que peuvent susciter les attentes du monde du travail. La jeune femme suppose initialement que les problèmes qu'elle rencontre dans son activité sont à mettre en lien avec sa manière d'être, elle fait donc des efforts dans ce sens, en anticipant des demandes qui ne viennent pas. En l'occurrence, bien qu'elle soit apprentie, il ne lui est pas explicitement demandé de se substituer aux autres, notamment pour accomplir les basses besognes, mais elle présume que c'est ce qu'elle doit faire et surtout, elle le fait pour tenter de se faire admettre dans le collectif de travail.

Si certaines adaptent leur caractère pour plaire à leurs collègues, d'autres apportent un grand soin à leur tenue, cherchant à correspondre au maximum à l'image de professionnelle. Ainsi, Tiffany, une employée de commerce de 17 ans :

J'ai toujours eu un bon contact avec le patron. J'veux dire, j'me suis toujours habillée vraiment classique, noir et blanc, ou bien tailleur ou bien comme ça, pas comme les autres apprenties...

Ces différents éléments font apparaître des formes de conformité extrême à ce qui pourrait être attendu sur le lieu de travail, une forme de surinvestissement dans certains rôles assignés aux femmes: avoir une apparence impeccable et des compétences sociales. Dans cette mesure, l'hypercorrection rejoindrait la stratégie que Molinier a identifiée sous le nom de muliérité, celle-ci consistant «à retourner le rapport aux contraintes inhérentes à la division sexuelle du travail en faisant comme s'il s'agissait de choix librement consentis » (Molinier, 2000 : 40). Il s'agit en fait de conduites par lesquelles une femme s'efforce d'éviter les représailles, qu'elle craint en ne se conformant pas au statut de soumission 
qu'on attend d'elle. Le parallèle avec la muliérité suggère de comprendre l'attitude des apprenties qui adoptent une stratégie d'hypercorrection comme révélatrice du fait qu'elles sont conscientes de ce qu'elles sont censées faire dans un marché du travail organisé par le principe de la division sexuelle. Cette conscience les conduirait à faire leurs les attentes qui leur sont implicitement adressées (soumission, gentillesse, compétences relationnelles, soin de leur apparence).

Par ailleurs, il serait également intéressant de s'interroger sur le décalage entre attentes réelles du monde du travail et représentations erronées de ces jeunes travailleuses. Dans ce deuxième cas de figure, l'hypercorrection ne dénoterait pas uniquement une appropriation des assignations sociales, mais serait l'expression de leur extranéité au monde du travail. Dans un rapport au travail qui ne va pas de soi (G. Moreau, 2000), où l'enjeu principal est d'accéder au métier et au savoir-faire, l'hypercorrection prendrait d'autres formes, celle de l'hypertravail, du zèle, de la disponibilité extrême ou encore de l'auto-formation. Soulignons toutefois que les environnements de travail peuvent également contraindre les personnes à opter pour ce type d'hypercorrection (zèle, etc.) en mettant les collègues en concurrence, notamment.

L'hypertravail renvoie à la position particulière occupée par les apprenti.e.s en formation professionnelle (G. Moreau, 2000). Les filles en particulier doivent montrer qu'elles y ont leur place, car elle ne leur est pas acquise d'emblée, contrairement aux garçons pour lesquels le statut de travailleur fait partie intégrante de l'identité sexuelle. Craignant de ne pas obtenir cette place et donc de n'être pas reconnues en tant que travailleuses, les apprenties font des heures supplémentaires, arrivent en avance, travaillent le week-end. Cette ardeur au travail concerne tant les savoirs théoriques (cours particuliers, fiches de répétition) que pratiques (apprentissage par cœur de séquences de travail). Laetitia, une employée de commerce de 16 ans, accumule les heures supplémentaires pour faire face à la pression provoquée par un volume de travail important:

Moij'arrivais pas à gérer le stress, j'avais des cernes, je craquais [...] Je leur disais: « J'essaie de faire tout », même pendant mes pauses je restais là-bas pour faire leurs trucs...

L'auto-formation, autre type d'hypercorrection que j'ai évoqué plus haut, révèle plus spécifiquement l'enjeu attaché à l'accès à la transmission du métier: c'est lorsque l'encadrement est défaillant ou manquant que les filles organisent leur auto-formation, faisant preuve d'une énergie qui s'inscrit bien dans une stratégie d'hypercorrection. Stella, une esthéti- 
cienne de 17 ans, dont la formatrice est absente pour cause de maladie prolongée, se forme comme elle peut:

Quand j'avais personne [pas de client.e.s], j'avais du temps libre, ben j'essayais de regarder ce que faisait l'autre. Et puis, je suis assez visuelle, donc j'apprends vite.

Si l'hypercorrection apparaît comme une stratégie majoritairement utilisée par les filles, quelques garçons y ont également recours. Dans les 3 cas présents dans le corpus, il est intéressant de noter que la forme d'hypercorrection retenue par les apprentis est une conformité extrême avec les assignations liées à leur statut, soit les tâches de nettoyage et de rangement, ce dont témoigne Théo, un horticulteur de 16 ans :

S'il y a des moments où j'ai rien à faire, où personne me donne quelque choseà faire, j'prends le balai, j'passe un coup de balai, j'vais demander si on a besoin de moi...

On aurait affaire ici à des formes de muliérité choisies par des garçons ou du moins des stratégies apparentées à la muliérité. Ils investiraient ainsi le rôle qui leur est socialement assigné, du moins durant le temps de leur formation professionnelle. En optant pour une stratégie «féminine", ils illustrent l'assignation de genre à laquelle le statut d'apprenti les renvoie (voir Chapitre 8).

De façon générale, les stratégies visantle maintien dansla formation professionnelle se colorent selon le genre: aux garçons la virilitédéfensive, aux filles l'hypercorrection, teintée de muliérité. Cette répartition met en lumière le fait que les stratégies d'accommodation s'adaptent aux normes du travail. Ainsi, il sera attendu, en particulier dans les professions masculines, que les jeunes hommes adoptent des comportements virils, permettant d'affronter le danger, la peur et la souffrance, en ayant des attitudes de déni, de prise de risques ou de défi. Pendant ce temps, dans les professions féminines, les filles seront invitées à être "féminines", soit soumises, agréables et dotées de bonnes compétences relationnelles.

Paradoxalement, la division sexuelle du travail contraint les personnes à s'engager dans des orientations propres à leur sexe (Vouillot, 2002), tout en les amenant à adopter des comportements qui laissent penser que les assignations sociales, en particulier de genre, sont des choix. Les filles en particulier sont conviées à désirer ce qui leur est imposé, à faire des choix raisonnables (Vouillot, 2007) et à montrer des comportements renforçant cette illusion. Il s'agit là d'un apprentissage non seulement du travail professionnel, mais aussi de la vie. Leur 
surinvestissement dans certaines attitudes attendues par un marché du travail fortement ségrégué et organisé selon un principe de division sexuelle du travail est peut-être un signe précurseur de la manière dont elles investiront les «missions» confiées aux femmes d'aujourd'hui, la "conciliation famille-travail» par exemple. Là aussi, elles n'auront pas le choix et devront faire leurs l'injonction à concilier (Delphy, 2001).

\section{Les réponses spécifiques des personnes en situation pionnière}

En matière de stratégies utilisées, les personnes en situation pionnière se distinguent de leurs collègues inséré.e.s dans les professions traditionnelles pour leur sexe. Par comparaison avec ces personnes, les pionnières et les pionniers recourent plus à l'action, à l'endurance et à la réaction. De manière générale, elles utilisent plus les stratégies d'accommodation et autant les stratégies d'affirmation.

Les stratégies retenues rendent compte de l'insertion spécifique des personnes en situation pionnière. C'est en particulier le cas des jeunes femmes actives dans des métiers traditionnellement occupés par des hommes, qui ont à faire la preuve de la pertinence de leur choix. Comme déjà évoqué en ce qui concerne la souffrance, les mises à l'épreuve, parfois violentes, sont légion dans les milieux qui résistent à l'entrée des femmes (Eckert, 2007; Maruani et Nicole, 1989). Face à ces résistances, les pionnières optent pour diverses stratégies qui vont de l'autocensure à l'affirmation de soi, en passant par l'assimilation aux pratiques du groupe (Dallera et Ducret, 2004).

L'assimilation des pratiques du groupe n'est pas présente dans le corpus, probablement parce qu'à l'instar d'autres stratégies défensives, elle s'acquiert au cours de la socialisation professionnelle, qui n'en est ici qu'à ses débuts. Cette stratégie est un moyen pour les pionnières de s'intégrer au collectif de travail (Devreux, 1984), mais celui-ci étant masculin, la stratégie consiste parfois, de fait, à adopter des pratiques masculines : en assimilant les pratiques du groupe, les pionnières montrent «qu'elles acceptent les codes et usages des milieux professionnels. Cette attitude peut conduire les apprenantes à assimiler certaines pratiques masculines» (Dallera et Ducret, 2004: 20). L'adoption de ces pratiques masculines par des femmes (pionnières) s'apparente alors à une forme de stratégie de virilité défensive.

L'autocensure est une forme d'effacement, qui n'apparait pas non plus de manière spécifique dans le corpus, lequel, il faut le rappeler, ne visait pas initialement une analyse approfondie des parcours pionniers. En revanche, on retrouve avec les stratégies d'action et de réaction ce 
que Dallera et Ducret (2004) nomment «affirmation de soi ». Face à un environnement parfois hostile, les jeunes femmes doivent en effet faire leur place, s'affirmer, voire se confronter, comme Julie, une paysagiste de 16 ans :

[Un collègue] qui a dérapé. J'lui fais: «Ben, s'il te plaît, tu me parles mieux quoi! », il m'a dit: «Tu te crois où espèce de petite pétasse?" J'ai été dire à mon patron: "Vous trouvez normal qu'on me traite de pétasse?» J'ai toujours du répondant, s'il $\mathrm{y}$ a des trucs où je peux répondre, je réponds. C'est vrai que parfois je dépasse un petit peu..., mais je reste toujours polie. [...] Si je me laisse marcher dessus, j’sais que ça se dégrade beaucoup trop vite, donc en général je réponds. En général, ils aiment pas que je réponde.

Dans cet extrait, la jeune femme a réagi immédiatement aux propos de son collègue, puis a eu recoursà l'action en allant informer son employeur. Elle est consciente des difficultés liées à son statut de pionnière, et sait qu'elle doit jouer dans la marge très étroite entre affirmation de soi et effacement.

Les stratégies les plus marquées des personnes en situation pionnière sont les stratégies d'accommodation, en particulier l'endurance. Elles renvoient à l'important enjeu d'intégration pour ces personnes atypiques. Si, de manière générale, l'endurance est l'une des stratégies les plus adoptées par les apprenti-e.s, l'intégration est d'autant plus centrale, pour les personnes pionnières, qu'elle doit se faire dans un collectif de travail auquel il est difficile de s'identifier, parfois hostile et faisant preuve d'une forte résistance. Les jeunes en situation pionnière savent qu'elles et ils doivent tenir bon, traverser les mises à l'épreuve, si elles ou ils souhaitent se maintenir dans leur choix atypique. Les enjeux de ce maintien apparaissent clairement dans le témoignage d'Agnès, une peintre en carrosserie de 18 ans:

Chaque matin, je me levais, je me disais: «Ah, ça va aller mieux aujourd'hui.» Pis des fois c'est vrai que ça allait mieux, puis des fois, ça allait pas mieux [...] Ils [mes collègues] m'ont dit: «Ouais, on aurait bien pensé qu'avec tout ce qu'on t’a fait comme vacheries, t'aurais pas aimé et t'aurais arrêté, t'aurais abandonné et tout ça.»Pis, j'leur ai dit: «Je vais pas vous donner ce plaisir!» [...] En fait, je voulais leur prouver et leur fermer leur clapet une bonne fois pour toutes, mais j'ai presque réussi, j’ai presque réussi. 
L'endurance pour se maintenir dans une formation atypique est également relevée dans l'étude de Croisier (2002), qui parle de persévérance déployée dans le but de travailler à tout prix dans le secteur choisi. D'une certaine manière, elle se retrouve aussi chez Flahaut et Pennec (2008), qui soulignent les deux positions extrêmes caractéristiques des stratégies des pionnières: l'assimilation, ou intégration, et le retrait. Dansl'assimilation, on retrouve la notion d'adaptation aux normes dominantes (Dallera et Ducret, 2004), et on y décèle la dimension intégrative vue précédemment.

Enfin, divers travaux sur les insertions professionnelles atypiques font apparaître une dernière stratégie, celle du retrait total ou partiel de l'activité atypique (Croisier, 2002; Flahaut et Pennec, 2008). Pour Flahaut et Pennec(2008), le retrait des pionnières est une réponse aux manifestations d'hostilité émanant du collectif de travail ainsi qu'à la non-acceptation du choix atypique par l'entourage. Chez leurs homologues masculins, le retrait a davantage à voir avec le bas salaire et les faibles perspectives de carrière dans les professions traditionnellement investies par les femmes. Cette différence est également soulignée par Croisier (2002), qui rappelle que les femmes optent pour des emplois dans des domaines plus conventionnels, et que les hommes se réorientent vers des professions mieux rétribuées. Les stratégies de retrait ont donc tout à voir avec un retour vers une conformité de genre, et c'est bien en ce sens que les arrêts de formation devraient être réinterrogés.

\subsection{Derrières les stratégies défensives, des enjeux multiples}

L'analyse des entretiens effectuée avec les outils de la psychodynamique du travail permet de faire émerger des questions nouvelles. En effet, les spécificités de cette population influencent largement l'accès, le choix et l'organisation des diverses stratégies de défense.

\subsubsection{Pour se défendre, il faut être en position de le faire}

Le premier aspect qu'il s'agit de souligner est le fait que certaines stratégies, pourtant largement évoquées par la littérature, n'ont pas été retenues ici. Il s'agit des stratégies de concurrence déloyale, de clivage, d'individualisme et de collaboration à ce que l'on réprouve. Il semble que l'on peut rattacher l'absence de recours à ces stratégies au statut d'apprenti.e: les personnes en formation ne sont tout simplement pas en position (en termes de hiérarchie, d'ancienneté, de stabilité) de les 
déployer. Leur position de faiblesse fait qu'elles subissent les contraintes et la souffrance, plus qu'elles n'y participent.

Le faible recours à la virilité défensive ne peut pas s'expliquer uniquement par le statut, mais laisse penser que lorsque la socialisation professionnelle des jeunes sera plus avancée, ils et elles adopteront davantage ce type de stratégies de défense fonctionnant sur des ressorts de genre - la virilité défensive, mais aussi la muliérité. Une autre hypothèse que l'on pourrait avancer ici est le fait que ces stratégies sont des stratégies collectives, elles nécessitent l'adhésion de toutes et tous, mais aussi l'appartenance à un collectif. Or, les apprenti.e.s n'en font pas encore partie, elles et ils sont isolé.e.s et optent principalement pour des stratégies individuelles.

\subsubsection{Se dégager d'une situation médiocre}

Le deuxième aspect de cette synthèse est inspiré par ce propos de Dejours (2000b : 175) :

Si la fonction première des systèmes de défense est d'alléger la souffrance, leur pouvoir d'occultation se retourne contre ses créateurs. Car faute de connaître la forme et le contenu de cette souffrance, il est malaisé de se battre contre elle. A la fin, les stratégies défensives, en raison même de leur efficacité vis-à-vis de l'équilibre mental, s'opposent à une évolution qui pourrait conduire à une stabilité d'un niveau moins médiocre.

Cette recherche porte sur des jeunes qui ont rompu leur formation professionnelle. Malgré les nombreuses stratégies mises en place, parfois simultanément, parfois successivement, la souffrance n'a vraisemblablement pas été suffisamment allégée. Et les stratégies déployées n’ont occulté que provisoirement la réalité qui en est à l'origine. Elles ont néanmoins permis de faire évoluer leurs situations. En effet, l'ensemble des jeunes rencontré.e.s sont sorti.e.s de l'environnement qui participait de leur malaise. Si certain.e.s sont en attente d'une solution durable (nouvelle place d'apprentissage), d'autres ont pu se stabiliser. Globalement leur situation paraît moins médiocre. Si la rupture peut être comprise comme un échec momentané des stratégies de défense, elle peut donc, dans certains cas, être également considérée comme positive, puisqu'elle a permis d'échapper au contexte responsable de la souffrance.

Travailler sur les arrêts prématurés de formation professionnelle sous l'angle des stratégies de défense n'est pas chose aisée, car il est difficile d'interpréter les ruptures de contrats d'apprentissage. Dans les entretiens, 
la rupture est toujours un moment pénible, et ce notamment parce qu'elle fait ressurgir la peur de ne pas trouver de place d'apprentissage et de vivre dans la précarité. Mais souvent, elle est également vécue comme un moment libérateur, qui permet de quitter une situation génératrice de souffrances. La rupture a en outre offert à certain.e.s l'occasion de faire le point, de se réorienter. Enfin, plusieurs jeunes ont laissé entendre que la souffrance leur a permis de se construire, de se renforcer face au monde des adultes et du travail. Leur souffrance a en quelque sorte été créatrice (Dejours, 2000b; Maranda et Leclerc, 2000). Ainsi, la rupture d'apprentissage lue à travers le prisme de la psychodynamique du travail peut être comprise comme une expérience positive, dans laquelle les jeunes ne sont pas uniquement des victimes, mais aussi des actrices et des acteurs.

\subsubsection{Arrêter sa formation, une stratégie de défense?}

Un troisième aspect que ce chapitre a fait ressortir est que l'arrêt de formation peut constituer en lui-même une stratégie de défense. Dans certains cas, notamment lorsque les personnes éprouvent une souffrance éthique (voir Chapitre 6), l'arrêt peut être une solution éthique, la personne pouvant à nouveau être en accord avec ses propres valeurs. L'interprétation de l'arrêt comme stratégie défensive s'est imposée en grande partie grâce aux analyses de genre. Ainsi, lorsque la tension entre les normes professionnelles et les normes de genre se fait trop vive, quitter sa formation ou son emploi peut apparaître comme une issue. Cette tension peut être éprouvée aussi bien par des jeunes effectuant des parcours professionnels typiques que par celles et ceux qui investissent un secteur ne correspondant pas à leur sexe. Dans ce cas, arrêter sa formation permet de résoudre le conflit lié à la transgression des normes. On retrouve cette interprétation de l'arrêt comme stratégie chez Molinier (2000) à propos du personnel soignant et de ses stratégies de mutation.

Les récits des pionnières ont permis de comprendre l'arrêt de la formation comme une stratégie de défense, tout en montrant que d'autres stratégies sont possibles pour faire face à la souffrance ressentie dans une situation de transgression. Notamment, si certaines quittent leur emploi ou quittent plus généralement le secteur atypique dans lequel la souffrance devient insupportable, d'autres s'accommodent de leur position de transgression en tentant de rendre leur pratique plus conforme aux normes sociales attachées à leur sexe, par exemple en «féminisant » leur représentation du métier, commeles mécaniciennes qui se qualifient «d'infirmières des automobiles» (Marry, 2007). Cette «féminisation» 
de la pratique peut également passer par une réorientation au sein d'un même champ professionnel, à l'instar de la paysagiste (Anne-Cécile) qui choisit de devenir horticultrice, un métier qui reste dans les métiers verts, mais dont la pratique est moins rude et où le taux de féminisation est plus important ${ }^{96}$.

Les jeunes hommes pionniers pourraient eux aussi utiliser l'arrêt comme une stratégie, mais pour de toutes autres raisons: se soustraire à un destin professionnel qui, parce qu' il est étranger à leur sexe, les menace dans leur identité sociale de dominant (Depoilly, 2014; Mauger, 2006). Sortir des métiers traditionnellement investis par les femmes peut ainsi être, pour les pionniers, une stratégie de protection contre les risques de déqualification (Croisier, 2002; Thiébaud, 2004). Le recours à cette stratégie pourrait même être encouragé par les professionnel.le.s de l'entourage, soucieux de l'avenir de ces jeunes hommes en insertion aty pique (Imdorf, 2007a). Il s'agit de faire en sorte que ces derniers se maintiennent dans une position dominante, propre à leur statut masculin. Compte tenu que tous les apprentis, quelle que soit leur insertion professionnelle, ont un statut subordonné dans la hiérarchie de l'entreprise, on pourrait faire l'hypothèse que non seulement les pionniers, mais également les apprentis insérés dans des professions traditionnelles, utilisent l'arrêt pour sortir d'une situation impropre à leur sexe. Quitter sa formation professionnelle signifierait ainsi le refus du statut subalterne qui leur est assigné en tant qu'apprenti. En d'autres termes, l'arrêt serait une stratégie qui met à distance la socialisation professionnelle au cours de laquelle on apprend les hiérarchies de l'entreprise (P. Kergoat, 2006).

Enfin, l'arrêt comme stratégie peut également être la réponse à une stratégie de virilité défensive qui aurait échoué. Encouragé par ses pairs à retourner son rapport subjectif aux contraintes en optant pour des attitudes de défi et de déni de la peur ou de la souffrance (Molinier, 2000), le jeune homme qui n'y parvient pas doit se dégager de la situation. Car son « échec» met à la fois en question sa virilité, à propos de laquelle il pourra être raillé, et la cohésion du collectif défensif. En réaction, celui-ci peut faire en sorte que la personne non conforme "craque et s'en aille ou tombe malade» (Molinier, 2000:30). Comme déjà évoqué, la virilité comme idéologie de métier nécessite donc l'adhésion de tous.

96 Bien que minoritaires, les filles représentent tout de même près d'un tiers de l'effectif dans cette profession: http://www.vd.ch/fr/themes/formation/ orientation/professions/zoom-sur-les-metiers/liste-des-emissions/horticulteur-horticultrice/ 
L'arrêt comme stratégie de défense face aux contraintes de genre ne doit pas faire perdre de vue qu'il existe d'autres stratégies, qui composent avec ces normes (virilité défensive, muliérité) et permettent le maintien en formation professionnelle. Si l'arrêt permet à certaines personnes de signaler leur refus des normes auxquelles on les assigne, cela ne signifie pas pour autant que les personnes qui se maintiennent en formation y adhéreraient. 


\section{Chapitre 8 \\ La formation professionnelle : un lieu de fabrication du genre ${ }^{97}$}

Ce chapitre examine la question de la transmission des normes de genre, soit la féminité et la masculinité, durant la formation professionnelle. Peu nombreuses dans ce champ, les recherches menées dans une perspective de genre se sont surtout penchées sur la ségrégation horizontale et sa perpétuation dans les choix professionnels des adolescent.e.s (Gonon, Haefeli, Heikkinen et Ludwig, 2001). Quelques travaux ont mis l'accent sur les discriminations subies lors de la sélection pour une place d'apprentissage (Imdorf, 2004, 2007b; P. Kergoat, 2014). D’autres, plus récents, ont analysé les stéréotypes en vigueur du côté des enseignant.e.s professionnel.le.s, et ainsi abordé la question du curriculum caché et de la transmission (Pfister Giauque et Flamigni, 2010).

La plupart des travaux sur le curriculum caché se sont concentrés sur l'école secondaire, voire primaire (Baudoux et Noircent, 1997; Duru-Bellat, 1995c; Solar, 1993). Ils ont fait émerger ce qui était transmis en termes de normes de genre tout au long de la scolarité, mais en marge du curriculum officiel. Les contenus « cachés» de l'enseignement, les attitudes des enseignant·e.s, les interactions ont ainsi été analysées, afin de comprendre comment, au sortir de la scolarité obligatoire, les

97 Le terme de fabrique ou de fabrication du genre a été retenu pour rendre compte d'un processus complexe. Des normes de féminité / masculinité sont transmises au cours de la socialisation professionnelle, certaines sont le prolongement des apprentissages faits au cours de la socialisation primaire et secondaire, d'autres sont inédites. En effet, il s'agit au terme de la formation professionnelle de produire des travailleurs et des travailleuses genré.e.s. Cette fabrication de futur.e.s travailleurs et travailleuses se fait de façon souvent violente, l'injonction à la conformité est fréquemment brutale. Ces termes de fabrique ou de fabrication sont également pris dans le sens du doing gender (West et Zimmerman, 1987). En effet, face aux injonctions, les apprenti.e.s ne restent pas sans rien faire. Elles et ils participent à cette production, en se montrant conformes aux attendus sociaux, en s'y opposant ou en proposant leur propre définition de la féminité et de la masculinité et en la confrontant à celle à laquelle elles et ils sont assigné.e.s durant leur formation professionnelle.

La deuxième partie de ce chapitre a fait l'objet d'une publication (Lamamra, 2011). 
jeunes femmes et les jeunes hommes avaient intériorisé des «savoirs »si différents ${ }^{98}$. A ma connaissance, rien de tel n'a été fait pour la formation professionnelle. Or, et compte tenu des éléments mis en évidence par les recherches évoquées ci-dessus (forte ségrégation, discriminations), il semble particulièrement intéressant de s'y arrêter. Pour ce faire, différents aspects seront examinés.

En premier lieu, le statut d'apprenti.e sera au centre de l'attention. L'analyse sera nourrie par les réflexions que Devreux (1992) avait menées sur les appelés de l'armée française. En effet, certains points communs avec la situation des recrues peuvent être mis en évidence. Par sa position hiérarchique au sein de l'entreprise, sa disponibilité face aux autres membres du collectif de travail, le faible contrôle de son emploi du temps (puisque là pour apprendre et travailler sous les ordres des collègues) et enfin l'assignation à certaines tâches, l'apprenti.e - comme le jeune soldat - a un statut qui le renvoie du côté des dominé.e.s et rappelle celui des femmes face au travail domestique. L'expérience de la formation professionnelle peut donc être analysée comme une incursion du côté du féminin, et ce indépendamment du sexe.

Dans un deuxième temps, les normes transmises en matière de masculinité et de féminité, ainsi que la manière dont se déroule cette transmission seront analysées. Pour les jeunes hommes, la transmission des normes du masculin sera examinée principalement en situation d'insertion traditionnelle, soit dans des métiers jugés conformes à leur sexe, mais quelques éléments de réflexion seront également proposés à partir du cas des pionniers. En situation d'insertion traditionnelle, soit dans des univers majoritairement occupés par des hommes, il s'agit de souligner le rôle central du collectif de travail, qui transmet les «trucs" pour faire face au danger et à la peur, qui contrôle la conformité de genre et fait peser la menace du déclassement ${ }^{99}$. Lapprentissage de la division sexuelle du travail et de la position provisoire de dominé permet

98 Les effets du curriculum caché sont saisissants : les filles sortent du secondaire en ayant tendanciellement de bons résultats, une faible estime d'elles-mêmes et une faible confiance en soi, pendant que les garçons ayant des résultats moins brillants, ont une confiance en soi et une estime d'eux-mêmes assez solides. Les filles ont en outre appris à se taire, pendant que les garçons se sont habitués à la prise de parole et à l'occupation de l'espace de la classe. Enfin, les jeunes ont appris une forme de division sexuelle du travail au sein de la classe (rangements, nettoyages) et dans les activités d'apprentissage, notamment lors des travaux de groupe.

99 Comme au Chapitre 3, la notion de déclassement permet de rendre compte de la mobilité descendante d'une classe de sexe vers l'autre. Ailleurs, et en 
aux apprentis de prendre conscience de ce à quoi ils doivent échapper. Les normes de la féminité seront ensuite analysées, tout d'abord lors d'insertions dans les professions traditionnellement féminines, puis, plus longuement pour les situations pionnières. L'accent sera mis sur la façon dont il est rappelé aux filles insérées de façon non traditionnelle, qu'elles ont transgressé et le "prix" qu'elles doivent payer (Marry, 2007). La mobilité de genre ${ }^{100}$ est en effet très subversive, en particulier pour les filles qui opèrent ainsi un mouvement ascendant, et entraîne des remises à l'ordre parfois brutales. L'analyse de la transmission de la féminité aux filles et de la masculinité aux garçons a été privilégiée, car elle se fait de manière explicite. L'intégration de normes féminines par des garçons ou de normes masculines par des filles (le recours par des pionnières à certaines stratégies viriles, par exemple) semble dépendre d'un mécanisme non explicite ${ }^{101}$, qui ne se donne pas facilement à voir. Elle ne sera donc pas spécifiquement analysée ici.

Enfin, dans une dernière partie, la transversalité du genre (Daune-Richard et Devreux, 1992) sera examinée. Plus exactement, il s'agira d'analyser la façon dont les normes apprises au travail se répercutent sur la sphère hors-travail. L'apprentissage de la division sexuelle du travail et de la hiérarchie entre les sexes s'exporte hors du milieu professionnel jusque dans la vie privée. D’autres aspects (liés à l'hétéronormativité) s'acquièrent et influencent les rapports entre les sexes. Les relations au travail mettent en scène des rapports sociaux de sexe antagonistes, conflictuels, pendant que les interactions entre les mêmes individus, mais à l'extérieur de l'entreprise, jouent sur un tout autre registre: celui de la "complémentarité entre les sexes». Ainsi, les

guise de synonyme, j’utiliserai la notion de mobilité descendante (voir note suivante).

100 J'emprunte la notion de mobilité de genre à Guichard-Claudic (2008) qui l'utilise pour traduire la mobilité sociale opérée lorsqu'une personne choisit une orientation professionnelle non traditionnelle.

101 Comme ce chapitre le montrera plus loin, la transmission de normes de genre lors d'une insertion traditionnelle se fait généralement de façon explicite. Les jeunes sont confronté.e-s par les professionnel-le·s du métier à ce que signifie le féminin ou le masculin dans un secteur donné. Lors d'insertions non traditionnelles, les pionnières ou les pionniers ne sont pas nécessairement socialisé·e·s aux normes de genre destinées aux personnes de l'autre sexe. Cependant, elles et ils baignent dans un environnement, dont elles et ils empruntent parfois les «outils». Ainsi, on n'attendra pas nécessairement d'une pionnière qu'elle se comporte comme un garçon - au contraire, par contre cette-dernière confrontée en permanence aux stratégies viriles de ses collègues, pourrait les faire siennes. 
relations entre individu.e.s se reconfigurent-elles selon que l'on se trouve sur le lieu de travail (en concurrence) ou en dehors (en complémentarité). Ces deux types de relations apparaissent en effet indispensables au maintien du système de genre, lui-même étroitement imbriqué au système hétéronormatif (Jackson, 2009).

\subsection{Apprentissage de la division sexuelle du travail et de la hiérarchie entre les sexes}

Le premier élément sur lequel portera l'a nalyse est l'apprentissage par les filles et les garçons de la division sexuelle du travail (D. Kergoat, 2000) et de la hiérarchie entre les sexes. L'analyse est largement inspirée des réflexions menées par Devreux (1992) sur les appelés de l'armée française. Il s'agit de comprendre comment se construit la position masculine dominante et la position féminine dominée, et dans quels espaces sociaux cette hiérarchie est produite. L'enjeu est de montrer comment les rapports sociaux de sexe organisent tous les espaces, y compris les lieux non-mixtes (ici, la formation professionnelle, chez Devreux, l'armée), soit des lieux où les deux catégories de sexe ne sont pas en présence l'une de l'autre. Cette expérience est commune aux filles et aux garçons.

Dans sa démonstration, Devreux soulignel'importance des espaces non-mixtes pour l'apprentissage des normes de genre. Welzer-Lang (2000) évoque la "maison des hommes" pour décrire la socialisation monosexuée des garçons, dans des espaces et des temps confinés d'entresoi. Les jeunes hommes y apprennent la masculinité par le contact avec leurs pairs, sans qu'aucune place ne soit laissée aux différences. La construction de la masculinité se fait par opposition au féminin, qui est utilisé comme un repoussoir, et par rejet de ce qui y est associé, en particulier l'homosexualité.

Moulin en propose un pendant pour les femmes, l'«espace homolatique féminin» (Moulin, 2005). Dans cet environnement, «le groupe d'amies constitue un espace d'apprentissage ou d'ajustement, de l'être féminin [...] Tout comme dans la maison des hommes, les différences n'ont de place que dans les relations sociales entre les sexes; être différente des personnes du même sexe effraie, catégorise, isole de l'intimité homolatique» (Moulin, 2005: 103-104). La construction de la féminité, bien qu'elle se fasse en antithèse à la masculinité, n'utilise pas le processus de mise à distance décrit plus haut pour les garçons. Le masculin apparaît dans l'espace homolatique féminin comme "autre", différent, et non comme figure-repoussoir. Au contraire, c'est le regard 
masculin qui évaluera in fine la conformité des jeunes femmes aux normes de la féminité.

Un espace social n'ayant pas besoin d'être mixte pour produire du genre, l'approche de Devreux est pertinente pour analyser la manière dont la formation professionnelle produit et transmet les normes de genre. L'objectif est également de montrer - c'était d'ailleurs le second but de Devreux (1992) - la transversalité des rapports sociaux de sexe. Ainsi, ce qui se construit durant la formation professionnelle en parallèle à la socialisation professionnelle, ou plutôt au travers d'elle, a des répercussions dans d'autres espaces sociaux que le travail, comme l'illustrera la troisième partie du chapitre.

\subsubsection{L'assignation aux tâches d'entretien}

Dans un premier temps, l'apprentissage des rapports de pouvoir et de la division sexuelle du travail se fait au travers de l'assignation aux tâches subalternes, d'entretien et de ménage, considérées commele «sale boulot » (Hughes, 1996; Lhuilier, 2005), car pas considéré comme directement productif et associé aux tâches domestiques. Cette organisation du travail illustre la position de subordination des apprenti.e.s dans la hiérarchie professionnelle et les confronte à leur position respective dans les rapports sociaux de sexe. Ainsi, dans les univers de travail, l'assignation $\mathrm{au}$ «sale boulot» renvoie les jeunes femmes à leur «destin» de femmes, pendant qu'elle sert de repoussoir aux jeunes hommes.

La position de subordination des apprenti.e.s est à mettre en lien avec leur statut ambigu, maintes fois évoqué dans le présent travail (voir Chapitres 1, 5 et 6). Pris.es entre formation et production, elles et ils sont fréquemment isolé.e.s, se situent tout en bas de la hiérarchie de l'entreprise, et se voient de ce fait confier les tâches ingrates ${ }^{102}$. Les activités d'entretien, de nettoyage, de rangement composent une part non négligeable du travail d'apprenti.e et prennent une place importante dans leurs griefs. Le lien entre tâches d'entretien et statut subalterne est très clairement présenté par Jeremy, un gestionnaire de commerce de détail de 20 ans :

102 L'analyse que l'on peut faire de ces activités diffère selon le contexte dans lequel elles prennent place. En effet, si dans un certain nombre de cas elles empêchent les jeunes d'apprendre le métier (voir Chapitre 5), l'entretien du matériel peut faire partie de la formation au métier. Certaines tâches subalternes permettent une participation périphérique au travail (Lave et Wenger, 2002) et font accéder les néophytes à une compréhension globale des activités (Billett, 2002). 
Y avait jamais personne, j'ai jamais rien appris [...] En fait, je suis un bouche-trou pour lui! Voilà c'est ce qu'il m'a dit. [...] Bah! C'est toujours moi qui devais faire les lavages et tout ça quoi!

Le jeune homme souligne ainsi que les activités de nettoyage l'empêchent de se former correctement.

Bien que les tâches d'entretien soient effectuées par les apprenti.e.s indépendamment de leur sexe, la question du ménage se retrouve plus fréquemment dans les griefs masculins. Que ce soit de manière revendicative ou de façon ironique, comme le fait Sébastien, un assistant en pharmacie de 16 ans :

Et puis la poussière, aussi. On me disait que j'étais assez fort [pour faire] la poussière! Ouais, je devais nettoyer les étagères.

Bien que ce ne soit jamais formulé aussi clairement que chez les jeunes appelés rencontrés par Devreux (1992), la résistance à ces tâches semble provenir autant de leur caractère subalterne, inintéressant et non formatif que de leur apparentement aux tâches domestiques, auxquelles les hommes échappent traditionnellement grâce à leur position dans les rapports sociaux de sexe. Or, durant l'apprentissage le statut d'apprenti.e-comme celui d'appelé à l'armée - semble prévaloir sur l'appartenance de sexe. Provisoirement, durant la formation professionnelle, filles et garçons sont astreint.e.s aux mêmes tâches, tâches considérées comme féminines lorsqu'elles sont effectuées dans d'autres sphères. Pour les jeunes hommes, cette assignation est provisoire, puisqu'elle ne dure généralement que le temps de l'apprentissage. Elle sert pourtant de repoussoir, l'enjeu étant de ne pas se retrouver en position de dominé, soit de devoir effectuer des tâches connotées comme féminines. La menace du déclassement leur apprend à tenir leur position dans le rapport social entre les sexes. Agusto, un dessinateur en bâtiment de 18 ans, en donne un exemple:

Il [le patron] le disait tout le temps [que je travaillais mal], il m'a même dit une fois que je ferais mieux de chercher dans un emploi de balayeur ou quelque chose comme ça, dans le ménage quoi!

La menace est celle d'un double déclassement social: celui d'occuper une profession dévalorisée et d'effectuer une mobilité de genre que l'on pourrait qualifier de descendante. 
Le lien entre tâches d'entretien au travail et ménage dans l'espace domestique n'est pas verbalisé par les jeunes hommes de notre corpus, mais par les jeunes femmes. Pour l'évoquer, elles utilisent fréquemment la figure de la femme de ménage. C'est le cas d'Agnès, une peintre en carrosserie de 18 ans:

Celui qui était censé m’apprendre le métier, il était plus dans ses trucs, parce qu' il avait jamais eu, enfin il a eu des apprentis, mais jamais une fille en plus... Il m'apprenait juste des trucs quand il avait du temps, doncj'apprenais vraiment rien. Donc c'était pas un apprentissage, c'était plus un truc de boniche qu'autre chose quoi.

$[\ldots]$

[Réponse à la question si les tâches d'entretien étaient liées à l'entreprise ou au fait d'être une fille dans un métier masculin] Les deux ouais, on était une fille ben, du coup c'est la conchita pis euh, eux et ben, s'ils prennent des apprenti.e.s c'est surtout pour le nettoyage quoij'crois parce que là, euh... Les personnes qui sont aux cours, ils ont tous déjà giclé, ils ont tous déjà fait quelque chose, moi à part nettoyer, rien du tout quoi.

Cet extrait réunit les différents éléments évoqués jusqu'ici: la question du statut d'apprenti.e qui permet certaines formes d'exploitation, dont l'assignation à des tâches subalternes, le conflit entre la réalisation de ces tâches et la possibilité de se former, la dévalorisation de ces tâches, qui apparaît très clairement dans les termes utilisés («boniche », « conchita») et enfin, le fait que les tâches d'entretien soient associées aux tâches domestiques effectuées majoritairement par des femmes. Dans le cas précité, la situation est encore renforcée par l'insertion pionnière, l'assignation permettant de rappeler la double situation de domination (apprentie et femme dans un métier «masculin»).

Les activités d'entretien effectuées dans le cadre du travail peuvent être vues comme le prolongement du travail domestique. Or, dans certains cas extrêmes, l'assignation à ces activités témoigne d'une situation d'exploitation, comme dans l'extrait suivant, où Elisabeth, une spécialiste en restauration de 17 ans, doit faire le ménage de sa patronne:

A un moment donné je devais toujours aller faire le ménage chez elle. Je passe l'aspirateur... chez la patronne. Elle habite en dessus. Je passel'aspirateur, la poussière, arroser les fleurs... 
Cette situation signale à la jeune femme d'une part qu'elle occupe une position dominée dans le rapport qui la lie à sa patronne, et d'autre part, que travail domestique au domicile de la patronne et tâches d'entretien sur le lieu de travail s'inscrivent dans le prolongement l'un de l'autre. Ainsi, le travail d'entretien exigé dans un rapport de travail et fait dans la sphère hors-travail est l'extension du travail domestique, auquel en tant que femme elle est traditionnellement assignée. Le rapport d'exploitation renvoyant aux statuts respectifs de patronne et d'apprentie repose entièrement sur la division sexuelle du travail. Ce cas laisse apparaître que c'est la position dans le rapport hiérarchique, ici le rapport de classe, qui organise les tâches ${ }^{103}$.

L’assignation à des tâches connotées comme féminines et dévalorisées permet donc aux jeunes femmes et aux jeunes hommes d'apprendre à la fois la division sexuelle du travail et la hiérarchie entre les statuts professionnels. Pour les jeunes hommes, cette expérience apparaît comme un moment particulier, qui ne devrait pas se reproduire, mais qui fait planer la menace de déclassement en cas de non-conformité à leur rôle de dominant. Les jeunes femmes, quant à elles, apprennent que leur position dans la hiérarchie entre les sexes les contraindra à exécuter ces tâches tant dans la sphère privée que professionnelle.

\subsubsection{Le travail d'apprenti·e, une activité fractionnée et subordonnée}

En plus de devoir assumer les tâches d'entretien et de nettoyage, les apprenti.e.s sont confronté.e.s à d'autres contraintes dues à leur statut et à leur position particulière dans l'entreprise. En formation, ces jeunes ne peuvent gérer leur emploi du temps : ce sont les tâches que leur attribuent la personne formatrice ou les collègues qui organisent leur journée. En outre, au bas de la hiérarchie del'entreprise, les apprenti.e.s sont au service de l'ensemble des autres employéee.s, et ce de façon plus marquée encore lorsque la situation est dysfonctionnelle et que leur statut n'est pas clair (main d'œuvre à bon marché). Ces jeunes sont donc disponibles pour toute tâche qui leur est demandée, même si celle-ci morcelle leur emploi du temps et désorganise leur gestion du travail. Cette disponibilité, qui parfois s'apparente à une corvéabilité à merci (commel'a montrél'extrait

103 Cet exemple montre que l'assignation à des tâches subalternes est bien liée à la position occupée dans un rapport social et non au sexe. Ainsi, les tâches dévalorisées sont attribuées aux personnes dominées. Cet exemple met également en lumière qu'au sein du même groupe de sexe, un rapport de classe (patronne-apprentie) organise le rapport social. 
ci-dessus), rappelle ce que Devreux (1992) a mis en évidence pour les appelés, à savoir leur non maîtrise du temps, fait de périodes d'attente, de répétitivité et de disponibilité. Ce phénomène se retrouve chez les apprenti.e.s, même si c'est dans une moindre mesure. Le fait de devoir être disponible à l'instant est souligné par Yasmine, une assistante dentaire de 16 ans :

On avait une sonnette pour appeler si on avait besoin d'aide par exemple. Une fois, il [le dentiste] m'a demandé d'aller chercher de la cire pour les dents, pour le détartrage. Je savais pas où elle était [...] donc j'ai fait long à la trouver. [...] Il a appuyé $15 ’ 000$ fois sur la sonnette...

Au-delà de la rapidité d'exécution, la disponibilité prend aussi la forme d'une contrainte à abandonner la tâche en cours pour répondre aux sollicitations d'un.e collègue. De ce fait, il n'est possible de gérer ni son temps, ni son activité, ce que vit Andrew, un ferblantier de 17 ans:

Par exemple, je sais pas, il [un collègue] me dit d'aller chercher un outil qui est à cinquante mètres, lui il tend le bras, il prend l'outil, pis moi quand je suis en train de travailler en haut il me fait: «Hé viens voir là, prends moi l'outil!»

$[\ldots]$

J: Si on me dit gentiment y a pas de problème. Si on me dit: «Oh p'tit con, va prendre ça!», comme on m'disait là-bas, non!

[...] Si je peux aider quelqu'un qui me demande... donc y a différentes formes. Ouais, gentiment: «S'te plaît tu peux faire ça pour moi, aide moi!», pas de problème. Mais: «Viens-là p'tit con, prends moi ça!», non!

Ces demandes révèlent très clairement la hiérarchie professionnelle. Le collègue peut se permettre de demander à l'apprenti d'interrompre sa tâche, de parcourir les 50 mètres qui les séparent pour aller chercher un outil, pourtant à sa portée, parce qu'il est son supérieur. Le manque de respect relevé par l'apprenti, souligne également cette relation asymétrique. Ces demandes ne sont doncqu'une façon de rappeler l'organisation verticale des rapports de travail.

Les éléments identifiés dans ces exemples (disponibilité, non gestion de son temps, morcellement des activités) sont autant de « caractéristiques du travail domestique tel que l'assument généralement les femmes dans la sphère familiale»(Devreux, 1992: 160). Ainsi, la ressem- 
blance avec le travail domestique ne se réduit pas à la nature des tâches, les travaux d'entretien, mais porte sur l'ensemble de l'organisation du travail des apprenti.e.s.

\subsubsection{Les règles du jeu: apprendre la hiérarchie et éviter le "sale boulot»}

Parallèlement à l'apprentissage de la division sexuelle du travail, les apprenti-e.s découvrent que celle-ci s'organise sur la base de leur statut (professionnel, mais aussi de sexe). Ainsi, si c'est leur position subalterne qui les contraint à exécuter le «sale boulot », ces jeunes vont comprendre qu'il suffit de «monter en grade » pour y échapper: dans l'entreprise, les salarié.e.s non-qualifié.e.s assument moins de tâches d'entretien que les apprenti.e.s, les salarié.e.s qualifié.e.s moins que les non-qualifié.e.s, les chefffe.s d'équipe moins queles salarié.e-s qualifié.e.s. Quant aux rapports entre apprenti.e.s, ils sont également structurés d'après l'ancienneté (ou selon leur année d'apprentissage). Cette hiérarchie est parfaitement tolérée par les entreprises formatrices, voire utilisée dans l'organisation du travail. Les apprenti.e.s de $1^{\text {ère }}$ année sont donc rapidement informé.e.s qu'années d'apprentissage, activités subalternes et tâches d'entretien sont étroitement liées. Hakem, un réparateur automobile de 19 ans, l'a appris auprès de ses collègues de cours:

En fait je crois que chez [nom de l'entreprise formatrice], j'avais entendu que les apprentis de $1^{\text {ère }}$ année, de $2^{\text {ème }}$, c'est plus le rangement, que aller voir un mécano qui est en train de démonter un moteur tout ça. C'est ce qui m’arrive aussi.

Cette répartition des tâches semble ici faire partie de la politique d'entreprise, être une étape dans la formation, un passage obligé. Parfois, elle est une manière de gérer et d'organiser le travail entre les apprenti.e.s et de signaler leur position hiérarchique respective selon leur ancienneté. D'autres fois, elle permet de privilégier l'encadrement de certain.e.s apprenti.e.s, notamment à quelques mois des examens finaux, comme le rapporte Nuño, un coiffeur de 18 ans:

J'ai fait plus de nettoyage que de coiffure en fait. Pis ça m'a, ça m’a... ça m'avait gonflé un peu [...] Au début, on fait partir sur les têtes, il m'disait de faire la barbe, j'avais commencé la barbe aussi. [...] Pis après il a changé d'avis, il m’a fait commencer à faire le nettoyage.

$[\ldots]$ 
J'étais un peu déçu, j'me disais: «Pourquoi ça serait toujours moi, on est trois apprentis? » Pis il a fait: «Bah, t'es le seul en première année, et eux ils travaillent alors, il faut bien que quelqu'un le fasse.»

\section{$[\ldots]$}

Je sais pas du tout en fait. Parce qu'on pense qu'il devait donner plus de temps aux autres apprentis qui étaient en troisième année.

L'organisation du travail dépend donc parfois du programme de formation: lorsque les examens finaux se profilent, certaines entreprises tendent à soutenir les apprenti.e.s de troisième année. Cependant, ces agissements répondent également à une autre motivation: le fait que les apprenti.e.s les plus âgé.e.s "travaillent», autrement dit, participent davantage à la production. On assiste ainsi non seulement à une hiérarchisation en termes d'âge, d'années d'apprentissage et d'expérience, mais aussi à la mise en évidence de l'opposition entre travail productif et travail non directement productif.

Echapper au travail de ménage et d'entretien, à la dévalorisation qui y est associée, est primordial. En effet, le travail non-productif renvoie - comme les tâches d'entretien évoquées précédemment - aux tâches domestiques effectuées majoritairement par des femmes. L'enjeu est donc pour les apprenti.e.s de quitter le statut le plus subalterne: celui d'apprenti.e de première année. Dès lors, elles et ils obtiendront un pouvoir, aussi minime soit-il, sur les personnes ayant un statut plus faible que le leur. Laetitia, une employée de commerce de 16 ans, fait état de son expérience avec l'apprenti de $3^{\mathrm{e}}$ année:

Il y avait un autre apprenti [...] Moi j'suis d'accord c'est à moi de faire tout ce qui est poubelle, vu que c'est ma $1^{\text {ère }}$ année [...] C'est rangement, tout [le travail] de $1^{\text {ère }}$ année, mais j'veux dire la manière qu'il m'a dit: "C'est moi le boss et pis c'est toi la bonne.» Moi j'ai fait: «Ecoute, tu ne parles pas comme ça!» Moi, je fais volontiers ... [les tâches]

Si la jeune femme, apprentie de première année, considère normal de se charger des tâches d'entretien, elle dénonce certains dysfonctionnements. Ce témoignage révèle les éléments sur lesquels repose la division du travail. Tout d'abord, le rapport de pouvoir s'est constitué sur la base de l'ancienneté dans l'entreprise : être en deuxième ou troisième année, soit être plus expérimenté·e, permet d'être déchargé.e de certaines tâches 
subalternes et donc d'échapper aux tâches de ménage. Ensuite, il ressort clairement que la division sexuelle du travail se trouve au cœur de ce rapport de pouvoir : non seulement exécuter des tâches d'entretien signifie être une femme, mais, pour l'apprenti de troisième année, mentionné ci-dessus par Laetitia, y échapper, c'est faire montre qu'il a cessé d'y être associé. Les termes utilisés sont d'ailleurs révélateurs du rapport de pouvoir (boss/bonne) et de la division sexuelle du travail, qui s'appuie également sur la division des sphères d'activité (le monde du travail «le boss» face à l'univers domestique «la bonne»).

Cet exemple montre que division sexuelle du travail et stratégies pour y échapper s'apprennent en parallèle (Devreux, 1992). En étant assignés aux tâches domestiques en tant qu'apprentis, les garçons apprennent que la meilleure manière d'y échapper est de quitter ce statut subalterne. Ainsi, «l'apprentissage du travail domestique [...], de la hiérarchie des fonctions et des moyens de se dégager des tâches ingrates en trouvant quelqu'un de plus inférieur ou plus dominé que soi pour les assurer, a pour arrière-plan la division sexuelle du travail expérimentée dans le cadre familial» (Devreux, 1992: 163).

Les apprenti.e.s font donc ensemble (dans les professions mixtes) ou séparément (dans des professions fortement sexuées) l'expérience de la division sexuelle du travail. Elles et ils apprennent ainsi que leur position dans les rapports sociaux de sexe est asymétrique. Le travail domestique et les tâches d'entretien ou de ménage dans l'entreprise formatrice étant dans le prolongement l'une de l'autre. Considérées comme non directement productives ${ }^{104}$ et dévalorisées dans la sphère professionnelle, ces tâches sont semblables à celles, non reconnues et non rémunérées, de la sphère domestique, majoritairement effectuées par les femmes ${ }^{105}$. Ainsi, être chargé.e du travail d'entretien, être soumis.es au morcellement du travail, devoir être disponible en permanence, ne gérer ni son temps ni

104 Le travail est qualifié ici de non directement productif pour faire état de la représentation encore largement partagée qui associe travail d'entretien (des biens et des personnes) et travail improductif. Cependant, les économistes, notamment féministes, mettent en valeur la part du travail domestique dans la production de la richesse nationale (PIB). Voir à ce propos les différents articles proposé par le numéro de Nouvelles Questions Féministes consacré à l'économie (Alvarez et al., 2007).

105 Les données de l'OFS (2008b) rappellent que les femmes consacrent plus de temps que les hommes à la plupart des activités ménagères et familiales. Ce sont elles qui effectuent en outre la plus grande part des travaux de nettoyage, puisqu'elles prennent ces tâches en charge à hauteur de 7,5 heures par semaine contre 1,5 heures pour les hommes. 
ses tâches sont autant d'éléments qui mettent les apprenti·e.s du côté du féminin. Ayant compris que cette situation était le fruit d'un rapport de pouvoir, ces adolescent.e.s vont chercher à s'en dégager en quittant le statut qu'elles et ils occupent. Cette réaction est plus marquée chez les garçons. En effet, ces derniers se montrent davantage révoltés par une situation, qui les place dans une position de dominé qu'ils ne sont pas habitués à avoir. Cet état de fait débute avec l'entrée en formation professionnelle et se maintient jusqu'à ce qu'ils trouvent plus dominé.e.s qu'eux (des apprenti.e.s plus jeunes ou des femmes). Dénoncer leurs conditions de travail leur permet non seulement de signaler qu'ils veulent se défaire de ce qui les maintient dans cette position dominée (statut, tâches), mais encore de rappeler qu'en tant qu'hommes ils ont vocation à être du côté des dominants, avec les avantages que cela comporte (échapper $\mathrm{au}$ « sale boulot»).

Durant leur expérience en formation professionnelle, les garçons auront fait une incursion du côté des dominé.e.s et des tâches auxquelles cette position contraint. Cependant, ils auront également compris qu'il suffit d'occuper un autre rang pour y échapper. Lassignation à certaines tâches dépendant d'une doublehiérarchie de classe (patron·ne/apprenti.e.) et de sexe (homme/femme), ils comprennent qu'à terme, leur position sociale (de sexe, de statut professionnel) leur permettra de fairel'économie de certaines activités. Pendant ce temps, les filles apprennent que quelles que soient leurs stratégies, elles échapperont plus difficilement au «sale boulot ", puisque même si au fil de leur formation, elles pourront s'en décharger sur des apprenties plus jeunes, leur position dans le rapport social de sexe les assignera durablement au travail domestique.

\subsection{Socialisation aux normes de genre en formation professionnelle}

La formation professionnelle permet la transmission de normes de genre. Cette socialisation de genre fait partie dela socialisation professionnelle et se construit au quotidien dans les relations qu'entretiennent les apprenti.e.s avec leur environnement professionnel, collègues, employeur.e, clientèle. Les interactions quotidiennes créent du genre (West et Zimmerman, 2009), dans le sens où tout une série d'éléments signalent sans cesse ce qu'est la norme en matière de genre et sont transmis directement ou indirectement tout au long de la formation à un métier. Dans cette seconde partie, l'accent sera mis sur ce qui se transmet en matière de masculinité et de féminité, que ce soit en situation d'insertion tradition- 
nelle ou en situation pionnière. L'analyse montrera comment, tout en effectuant leur formation, ces jeunes apprennent à être des femmes et des hommes conformes aux attendus sociaux en matière de genre. Le regard sera également porté sur la manière dont les injonctions et les rappels à l'ordre en la matière sont faits. Enfin, des portraits-types de ce qui est attendu au travail en termes de féminité / masculinité seront proposés.

\subsubsection{Devenir un homme}

Dans ce corpus, les garçons sont majoritairement insérés dans des professions traditionnelles pour leur sexe $(\mathrm{N}=21)$ - dansl'industrie, l'industrie automobile, les métiers de l'artisanat, les métiers verts, la construction, la restauration, ainsi que dans des secteurs mixtes comme la vente ou les emplois de bureau. Deux jeunes hommes sont en situation pionnière, l'un étant assistant en pharmacie, l'autre coiffeur. Il s'agit donc de voir à présent quelles sont les normes transmises aux garçons durant leur formation professionnelle et comment se fait la transmission.

\section{En situation d'insertion traditionnelle}

Dans les métiers où les hommes sont majoritaires, l'injonction porte fréquemment sur la conformité aux normes de virilité ${ }^{106}$ et se fait souvent de manière assez brutale.

Patrick, un réparateur automobile de 16 ans, témoigne de la rudesse des rapports de travail:

Les insultes, j'avais l'habitude... [...] Donc j'me f'sais insulter tous les jours par mon collègue. Mais pas forcément très blessant, mais au bout d'un moment ça fait chier!

Ainsi, à côté de l'accoutumance des corps à la pénibilité physique des métiers, il semble y avoir une habituation des esprits à la brutalité, qui semble partie intégrante des univers professionnels masculins. L'un des ressorts de la virilité repose sur cette accommodation à la violence verbale, physique ou symbolique. Matthieu, un vendeur en pièces détachées de 16 ans, en souffre:

106 La distinction entre virilité et masculinité est faite dans le sens que lui attribue Molinier (2000). Face aux contraintes du monde du travail, de la souffrance provoquée par l'exercice de l'activité, la virilité sert de stratégie de défense et se construit sur une surenchère virile (attitudes de défi face au danger, non évocation de la peur et de la souffrance) et sur un mépris de tout ce qui est associé au féminin. 
Ben j'imaginais une image justement plus détendue, que les gensc'étaient des passionnés de voitures et pis que, c'était dans une bonne ambiance tout le temps. Mais en fait, je remarque que voilà, non, c'était pas tellement ça, [...] c'était rude, une ambiance rude, dans un garage, c'est pas ça...

Dans l'exemple précité, le jeune homme - vendeur de pièces détachées (col blanc) - prend ses distances avec ses collègues, afin de s'éloigner d'un modèle viril, qu'il associe au monde de la mécanique (cols bleus). Le jeune homme refuse de céder à la pression à la conformité virile; il se distancie de ces pratiques et de l'univers professionnel qui les produit (Eckert et Sulzer, 2007). A titre d'exemple, durant ses pauses, il va versles employé.e-s du magasin, vers les secrétaires, mais évite les mécaniciens. Il refuse d'entrer dans une dichotomie masculin valorisé - féminin dévalorisé et s'appuie pour cela sur une autre dichotomie: la distinction entre cols blancs valorisés et cols bleus dévalorisés, employéee·s et ouvriers/ouvrières.

Le déni de la peur et de la souffrance constitue le deuxième ressort de la virilité (Dejours, 1998; Molinier, 2000). Il s'agit d'apprendre à dépasser sa peur, à composer avec elle, comme le rappelle Jacques, un menuisier de 18 ans:

J'ai eu mon accident en décembre euh 2005. Là ça allait encore j'pouvais travailler sur les machines, pis tout d'un coup, j'sais pas, c'est venu d'un coup en février-mars, c'était venu pis à partir de là j'commençais à avoir de plus en plus peur... Et dès que la machine tournait, je pouvais pas m'en approcher. J'allais quand même travailler sur les machines, mais je faisais un p'tit truc, après j'avais les palpit' [palpitations] au cœur, je supportais plus. J’ai essayé de battre [ma peur], mais j'arrivais pas.

Cet apprenti tente d'affronter sa peur, encouragé en ce sens par le collectif de travail. Le formateur, les collègues l'encouragent à user des stratégies de défense viriles: «essaie de battre ta peur». Les encouragements sont accompagnés d'un signal clair de la part du patron, qui a tout d'abord «un peu tiré la gueule », signalant que la peur n'est pas admissible dans un univers masculin et que la virilité passe par la capacité d'en faire fi. Parfois cette injonction se fait de manière plus confrontative, soulignant l'importance de la conformité de genre au masculin. C'est le cas de Liam, un laborantin en chimie de 17 ans: 
Elle [la stagiaire qui le forme] me donnait des trucs à faire en vitesse, qu'il fallait envoyer aux Etats-Unis après et tout, fallait des tests, pis une fois je lui ai dit: «Mais vous trouvez pas que c'est un peu dangereux de donner ça à un apprenti de première année?» Puis je me suis presque un peu engueulé avec donc...

Ce qui ressort de ce témoignage c'est que dans le système viril, la peur, les doutes ne se disent pas. Dans l'univers professionnel de l'industrie, on doit apprendre à se taire. De la même manière que dans nombre de métiers où les hommes sont majoritaires, la pénibilité physique ne se mentionne pas. On se doit d'être fort, même si ce n'est jamais mentionné directement. Les propos de Théo, un horticulteur de 16 ans sont éloquents:

Faut porter la corbeille de terre et pis ça allait... Ouais, une main dessus pis comme ça, c'est... un p'tit peu lourd. Quand c'est des corbeilles qui ont une capacité de, j'sais pas, septante litres quelque chose comme ça... Alors quand c'est de l'herbe mouillée, c'est pas mal aussi... pour soulever... c'est voilà... $[\ldots]$

Ça va... mais moi je trouve encore assez sympa, j'trouve que c'est... Ouais, c'est assez sympa ça. Ouais, ben c'est assez dur, pis euh... Ouais, c'était cool.

Il souligne à la fois les astreintes physiques de sa profession, sa maitrise des gestes techniques ("une main dessus, pis comme ça»), mais encore le plaisir qu'il y trouve («c'est sympa, c'était cool»). On assiste ici à un processus d'inversion, la virilité étant de faire de la pénibilité un plaisir, celui d'affronter la matière, de la maîtriser (Dejours, 1998).

Là encore, le collectif de travail joue un rôle déterminant, en enseignant à la fois les moyens d'épargner son corps et l'interdiction de faire état de la douleur, de la souffrance:

Une fois qu'ils [les collègues] voient qu'on peine un p'tit peu, pour le dos surtout... Y'en a toujours un ou deux qui se marrent et pis qui vous laissent faire, ils rient... Autrement, ils expliquent pour les reins tout ça.

Les jeunes hommes apprennent donc en parallèle les gestes techniques, les «trucs du métier» et le déni de la souffrance.

Une certaine violence sous-tend ces apprentissages: les collectifs de travail masculins stigmatisent la personne «non conforme» en l'asso- 
ciant à la figure repoussoir de la féminité (voir Chapitre 7). Les attaques sur le déni de virilité sont donc une arme utilisée pour appeler les jeunes hommes à la conformité et ceux qui y dérogent sont qualifiés de femmelettes ou d'homosexuels. Alessandro, un peintre en carrosserie de 19 ans subit un certain nombre de remarques mettant en cause sa virilité:

J'en ai eu marre que mon chef me rabaisse toute le temps, pis pour une autre fois, il s'en est même pris à ma famille. Il a osé dire que mon père n'avait pas de couilles...

$[\ldots]$

Il me rabaissait. Il me disait des trucs: «manche à couilles», des choses comme ça, «bon à rien».

Ici, la violence prend la forme d'insultes à connotation sexuelle. Ainsi, apprendre la virilité, c'est apprendre à supporter les insultes, la rudesse, la peur, la pénibilité et la souffrance. C'est retourner les contraintes en plaisir et se distancier radicalement de tout ce qui est associé au féminin, à la faiblesse, à la fragilité. Le système viril ne supportant aucune faille, l'ensemble des hommes du collectif doit adhérer, se conformer. Les collectifs de travail jouent un rôle déterminant dans ces apprentissages, en enseignant les savoir-faire, mais aussi en rappelant à l'ordre ceux qui ne seraient pas conformes. Pour s'assurer de l'adhésion de tous, ils brandissent la menace de la non-reconnaissance au sein du groupe des hommes, et donc d'un déclassement de fait dans le groupe dominé des femmes.

\section{En situation pionnière}

La question des pionniers ne sera qu'esquissée ici. Très minoritaire dans notre population, leur orientation atypique est en outre le résultat d'un choix par défaut ${ }^{107}$.

En outre, l'accueil des pionniers dans les secteursà dominante féminine est généralement bon et fréquemment assez facile (Guichard-Claudic et al., 2008), leurs difficultés sont d'un autre ordre: ils regrettent le manque de perspectives, de reconnaissance et de possibilités d'avancement. Ainsi, ce n'est pas l'environnement professionnel qui rejette les hommes, mais ce sont ces derniers qui s'en échappent, faute de pouvoir y trouver de

107 La population n'ayant pas été sélectionnée dans le but de procéder à une analyse de genre, la question des pionnières et pionniers a été laissée au hasard. Il n'y a que 2 pionniers et le matériel ne permettrait pas d'analyse pertinente. 
quoi remplir leur rôle de pourvoyeur principal (Croisier, 2002 ; Thiébaud, 2004). Certain.e.s auteur.e.s ont constaté qu'en situation atypique, la division du travail se reconfigurait, certaines tâches étant assignées de préférence aux hommes dans les professions féminisées (Jaboin, 2010; Lada, 2008; Le Feuvre et Laufer, 2008). Rien de tel n'apparaît dans les entretiens effectués auprès des deux pionniers, probablement parce que ce sont des apprentis de première année, auxquels on ne confie pas encore de tâches spécifiques. Les seuls éléments qui transparaissent en filigrane dans notre matériel sont liés aux possibilités offertes par ces professions traditionnellement féminines. L'assistant en pharmacie signale le caractère répétitif et monotone de l'activité, qui laisse présager une profession offrant peu de perspectives de développement et d'avancement. Quant au coiffeur, il relève, comme bon nombre d'autres jeunes hommes de notre corpus, l'assignation aux tâches d'entretien et de nettoyage qu'il subit ${ }^{108}$.

Un aspect de la socialisation masculine pourrait en outre expliquer l'absence d'éléments intéressants sur la transmission de normes de genre aux garçons en situation atypique. Si l'on considère comme Welzer-Lang (2000) que la masculinité se construit prioritairement entre pairs dans «la maison des hommes», il n'est guère étonnant que de jeunes hommes insérés dans des univers professionnels majoritairement féminins ne soient guère confrontés à des rappels à l'ord re quant à leur conformité de genre. En effet, les hommes étant absents, il n'y a pas d'injonction à la conformité faite par des pairs. Par ailleurs, le féminin se construit dans l'entre-soi de l'espace homolatique féminin (Moulin, 2005), mais ne se construit pas par le rejet du masculin. Il n'y a pas, du côté des femmes, de construction d'une hiérarchie, mais simplement une "distinction ordinaire» (Moulin, 2005: 103). Ainsi, lorsqu'un jeune homme se retrouve en situation minoritaire, le groupe de femmes ne se sent pas menacé ${ }^{109}$ et ne lui renvoie pas d'injonction à la virilité, ni ne relève sa non-appartenance au groupe majoritaire, ici en l'occur-

108 Dans la coiffure toutefois, le nettoyage du salon fait intégralement partie du métier. Il est d'ailleurs effectué également par les patron·ne.s. La propreté du salon informe la clientèle du statut du salon (Benelli, Rosende et Messant-Laurent, 2008). On retrouve également dans cette pratique, la participation progressive au travail: de la participation périphérique à une participation entière (Billett, 2002).

109 Précisons toutefois que bien que la plupart du temps les professions féminisées accueillent le processus de mixité favorablement, certaines associations professionnelles s'en inquiètent (Le Feuvre et Laufer, 2008). En effet, les logiques de ségrégation font que les rares hommes occupent rapidement les postes de pouvoir au sein de ces professions, empêchant les femmes d'y 
rence le groupe des femmes. Au contraire, elles ont plutôt tendance à s'inquiéter du bien-être des pionniers (Imdorf, 2007a). Contrairement aux bastions masculins dont il faut forcer l'entrée, les métiers féminins accueillent la plupart du temps ces pionniers favorablement. L'arrivée de jeunes hommes dans des professions pensées «comme des espaces de relégation ou de marginalisation sociale» (Le Feuvre et Laufer, 2008) permet de revaloriser ces espaces. Il n'y a donc guère d'attentes à l'égard des jeunes hommes, si ce n'est de participer à rendre les professions plus mixtes. C'est avant tout cette mixité qui est mise en avant, comme un plus, comme une richesse. Ainsi, Sébastien, un assistant en pharmacie de 16 ans, relève qu'il a été retenu pour cette raison:

Comme c'est des nouvelles pharmaciennes responsables, elles m'avaient dit que c'est vrai que comme j'étais un garçon, ça les, ça les motivait bien...

$[\ldots]$

Elles ont dit ça, pis que ça, ouais que ça changeait par rapport aux filles quoi!

Cet extrait illustre l'une des raisons qui poussent certaines femmes à engager des jeunes hommes et à les faire entrer dans leur profession : la revalorisation du métier grâce à la présence masculine.

\subsubsection{Apprendre à être une femme}

La plupart des filles du corpus sont insérées dans des professions traditionnelles pour leur sexe $(\mathrm{N}=16)$ - la vente, les soins, certains métiers verts (fleuriste) et les professions mixtes comme les métiers de bureau et certains métiers de la restauration

Deux types de cas relèvent des parcours pionniers. L'insertion pionnière, soit l'insertion dans une profession majoritairement occupée par des hommes $(\mathrm{N}=7)$ et les situations pionnières, où les jeunes femmes font leur apprentissage dans une profession traditionnelle pour leur sexe, mais dans un environnement entièrement masculin $(\mathrm{N}=2)$. Les insertions pionnières concernent les professions suivantes: horticultrice, paysagiste, peintre en carrosserie et cuisinière. Les situations pionnières concernent une employée de commerce dans une entreprise de courtage et une gestionnaire de vente dans un magasin de matériel

avoir accès dans des secteurs où elles sont majoritaires et où jusque-là, elles avaient une des rares occasions de faire carrière. 
audio pour voiture. Dans les deux cas, le personnel est composé uniquement d'hommes. Ces deux jeunes femmes rencontrent ainsi sur leur lieu de travail des problématiques similaires aux pionnières. Aussi, les deux groupes ne seront-ils pas distingués pour analyser quelles normes sont transmises au cours de la formation professionnelle et comment la transmission se fait.

\section{En situation d'insertion traditionnelle}

En premier lieu, soulignons qu'un certain nombre d'injonctions sont faites aux jeunes femmes en matière de conformité physique. Travailler dans une profession traditionnellement occupée par des femmes demande de se soumettre à ces attentes normatives. Yasmine, une assistante dentaire de 16 ans, a ainsi appris qu'il s'agissait non seulement d'être motivée et compétente, mais aussi d'avoir le «physique de l'emploi» (Gasparini, 2007):

Y en a un [employeur] qui voulait pas me prendre à cause de ça [problèmes de poids suite à une maladie au cerveau] [...] Il a dit à la conseillère [aux apprenti-e.s] : «Ouais, j'sais pas si elle [arrive] bien à se bouger au fauteuil, en fait.» Ça c'est de la discrimination, j'veux dire, ça devrait être interdit. [...] Si maintenant on doit être mannequin pour avoir un apprentissage, ben je me demande où on va.

La profession d'assistante dentaire est une activité de représentation, pour laquelle il s'agit d'être attractive (mince, charmante, avec une hygiène parfaite). Ces exigences mettent sans doute en évidence les aspects informels de l'activité: outre l'assistance technique du dentiste, l'assistante participe également à la création d'un environnement rassurant pour les patient.e.s. Cette atmosphère repose notamment sur un «ordre naturel» entre les sexes, construit sur des représentations stéréotypées: un homme en position dominante (médecin, médecin-dentiste) et une femme qui l'assiste. Cette conformité aux normes de genre (dans la position occupée et dans l'apparence) est donc indispensable, car elle participe à la légitimation de la hiérarchie de l'entreprise et permet à l'organisation du travail au sein des cabinets dentaires de fonctionner de façon optimale (Imdorf, 2007a). Les assistantes dentaires devant correspondre parfaitement aux normes de genre attendues, il n'est donc guère surprenant de trouver un autre exemple d'injonction à la conformité physique dans cette même profession. Il concerne Leonora, ayant également 16 ans: 
J'ai appelé ma sœur en pleurant pace que l'hygiéniste m'a dit que j'puais [...] En fait c'est qui f'sait chaud, pis on avait un long traitement pis j'ai juste un peu transpiré... [...] Pis elle [l'hygiéniste] est v'nue pis elle m’a dit: « Prends le pas mal, mais tu pues!» Pis après elle est allée parler d'ça dans l'cabinet...

Il ressort de cet exemple qu'avoir «le physique de l'emploi » permet de se maintenir en formation. En outre, c'est également une condition pour espérer décrocher un premier emploi. Les jeunes femmes savent donc que pour travailler dans certains secteurs, elles doivent travailler leurs corps (Hidri, 2005). Cette norme est si fortement ressentie par les jeunes, que le projet principal de la première des deux assistantes dentaires évoquées ci-dessus est de perdre du poids, ce notamment pour trouver une nouvelle place d'apprentissage et un futur emploi.

Le choix vestimentaire peut également être compris comme un prolongement de la conformité physique. Dès lors, trouver la tenue adéquate, notamment lorsqu' il n'y a pas d'uniforme, peut se révéler complexe, comme le souligne Tiffany, une employée de commerce de 17 ans:

Je me suis toujours habillée vraiment classique, noir et blanc, ou bien tailleur ou comme ça, alors que les autres apprenties, c'est décolleté, t-shirt au-dessus du nombril, tout ce que je ne mettrais jamais pour aller bosser [...] Le patron m'a toujours dit: «Je te félicite, tu t'habilles mieux que l'apprentie de $3^{\mathrm{e}}$ et t'es plus serviable.»

Le choix de la jeune femme se fonde sur l'idée qu'elle se fait du «féminin professionnel». Celle-ci se construit sur une représentation stéréotypée del'image qu'il faut avoir dans les métiers de service et plus généralement dans les espaces de travail; elle s'appuie également sur la figure repoussoir des anti-modèles féminins, sans cesse évoqués par les jeunes femmes (Moulin, 2005). Il est nécessaire d'être attractive, mais pas vulgaire, pour

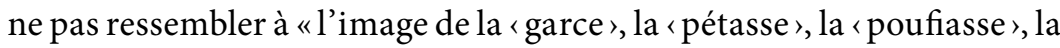
〈salope 〉, la 〈pute 〉» (Moulin, $2005:$ 175). L'image de professionnelle doit se forger en composant avec le double standard auquel sont confrontées les femmes ${ }^{110}$. La réaction du patron est en outre particulièrement intéressante. Il valorise comme une compétence la conformité de genre dont fait preuve la jeune femme. Il est en effet attendu d'elle, un «savoir-être

110 Par double standard, j'entends l'obligation faite aux femmes d'être séduisantes, voire séductrices, mais pas vulgaires. Les comportements jugés inadéquats sont stigmatisés et renvoient à la figure de la prostituée (Andro, Bachmann, Bajos et Hamel, 2010; Pheterson, 2001). 
indicible renvoyant implicitementà la construction sociale de la féminité: séduction, attrait pour les vêtements, souci du corps, diplomatie (Gasparini, 2007 : 144). La question de l'habillement est donc bien à comprendre comme un prolongement de la conformité physique, l'apparence jouant un rôle central dans le monde professionnel, en particulier dans certains métiers de service fortement féminisés.

En plus de l'apparence, le contrôle social représente un autre élément récurrent dans la socialisation professionnelle des jeunes femmes. En effet, les apprenties dénoncent plus que leurs collègues masculins l'intrusion de leur formateur ou de leur formatrice dans leur sphère privée. Sarah, une fleuriste de 16 ans, en donne un exemple:

Ouais, ils [le patron et sa compagne] m’avaient déjà dit que j'avais de mauvaises fréquentations, ce qui n'est pas vrai pour moi...

Dans ces cas, l'ambiguité du rôle de la personne formatrice apparaît. En effet, le patron susmentionné sort de ses attributions professionnelles, puisqu'il entre dans la sphère privée. Par contre, il reste dans son rôle d'homme protecteur, en ayant des réflexes paternalistes. La situation est amplifiée du fait du rapport dissymétrique induit par les statuts, l'âge, mais aussi le sexe. Ce faisant, il signale à la jeune femme qu'elle doit être protégée et donc qu'elle est faible. Ce même phénomène se retrouve de manière exacerbée auprès des pionnières.

\section{En situation pionnière}

Les difficultés, les motivations ou encore les stratégies des jeunes femmes ayant osé braver les bastions masculins ont été largement documentées (Daune-Richard et Devreux, 1992; Legault, 2001; Marry, 2007; Pfefferkorn, 2008).

Ce qui retiendra plus particulièrement notre attention ici est le double apprentissage de normes de genre que font les jeunes pionnières. D'une part, un certain nombre d'obstacles et de mises à l'épreuve leur sont imposés, pour leur signifier qu'elles ne sont pas les bienvenues dans cet univers non-mixte, qu'elles ne sont pas au bon endroit, en bref qu'elles ne sont pas des hommes. D'autre part, les attaques spécifiques dont elles font l'objet (plaisanteries sexistes, allusions sexuelles, harcèlement sexuel) les renvoient à une féminité stéréotypée et réductrice. Ainsi, les pratiques des collègues et des personnes formatrices leur indiquent à la fois ce qu'elles ne sont pas et ce qu'elles devraient être; en somme, ce à quoi elles devraient correspondre en termes de normes de genre. 
J'aimerais relever la brutalité des injonctions à la conformité. Les attaques sont ici particulièrement frontales et explicites, ce qui s'explique d'abord par leur sexe, mais aussi probablement par le statut et l'âge des apprenties ${ }^{111}$. Les pionnières doivent faire leurs preuves, montrer qu'elles ont leur place dans le métier, voire dans le collectif de travail, et prouver leur détermination. Ces mises à l'épreuve, souvent physiques, visent à mettre en scène la supposée incapacité des pionnières à faire face à la dureté de la profession choisie; elles sont en outre relayées dans le discours des collègues. Agnès, la peintre en carrosserie de 18 ans, l'apprend à ses dépens. Outre la mise à l'épreuve physique évoquée précédemment, elle est de surcroît attaquée de front:

Ils [mes collègues] m’ont dit: «Ouais, on aurait bien pensé qu'avec tout ce qu'on t'a fait comme vacheries, t'aurait pas aimé et t'aurait arrêté, t'aurait abandonné et tout ça.» $[\ldots]$

Au début, ça allait bien et tout et pis après j’ai commencé à être rabaissée, comme quoi de toute façon j'étais une fille que, ce métier c'était pas pour moi. Enfin les gros machos quoi! Que de toute façon les filles, c'était pas leur métier, que j'allais pas faire long dans la carrosserie, j'allais plus souffrir qu'un mec parce que j'étais une fille justement.

Comme elle n'est pas un homme, ces collègues lui contestent la possibilité d'être une professionnelle. Dans cet environnement, identité professionnelle et identité sexuée vont de pair. Ainsi, cette pionnière est face à un dilemme difficilement surmontable: soit elle est une non-femme, soit elle est un non-professionnel (Cassell, 2000) ${ }^{112}$.

Hormis la résistance, les femmes sont également confrontées à l'exclusion. Ce phénomène est assez typique des lieux de construction

111 C'est le caractère particulièrement frontal des attaques qui conduit à faire le lien avec l'âge et le statut d'apprentie. Cependant, les pionnières, y compris dans les professions prestigieuses comme la chirurgie, sont également confrontées à des remarques mettant directement leur présence en question (Cassell, 2000).

112 Pour reprendre l'exemple cité par l'une des chirurgiennes rencontrées par Cassell (2000: 73): «ou bien vous n'êtes pas une femme, mais un ours, un chien ou une lesbienne; ou bien vous n'êtes pas un chirurgien, un bon chirurgien». Cet exemple illustre en outre comment les normes de genre se fondent sur les normes de l'hétéronormativité, la non-femme étant notamment une lesbienne. 
de la masculinité, d'où le féminin est rejeté (Welzer-Lang, 2000). Sur le lieu de travail, il se concrétise par les mises à l'épreuve présentées précédemment et par la mise à l'écart des pionnières (Dallera et Ducret, 2004). Laetitia, une employée de commerce de 16 ans qui travaillait chez des courtiers, en a fait l'amère expérience:

Parce que j'suis une fille, j'suis coincée [...] Avec lui [l'autre apprenti], ils [les collègues] avaient des trips tout ça, moi je veux dire, ils me laissent dans mon coin. C'est salaud, mais c'était comme ça, j'sais pas. [...] On avait que des hommes à part une secrétaire et pis moi...

Etre une femme est un obstacle à son intégration dansl'entreprise, tandis que son collègue masculin ne semble, quant à lui, ne pas rencontrer de difficultés particulières. Cette situation a certainement des répercussions sur la transmission des savoirs par le collectif de travail.

Autre phénomène, la surprotection (Dallera et Ducret, 2004), dont font preuve les employeur.e.s lors de l'arrivée d'une pionnière dans une équipe. En cas de difficultés, elles et ils optent parfois pour une attitude protectrice, voire paternaliste, qui entend mettre les jeunes femmes à l'écart du danger, notamment quand des violences se sont manifestées au préalable. C'est le cas de Julie, une paysagiste de $16 \mathrm{ans}^{113}$, qui suite à un accident provoqué par un de ses collègues (il a taillé la branche sur laquelle elle était assise), se voit restreindre sa marge de manœuvre:

Après [l'accident], j'avais plus le droit non plus de grimper aux arbres, pour les tailler. Avantj'pouvais, hein. Maintenant: «Non, non, t'as plus le droit, t’as plus le droit! T'as plus le droit de monter sur la machine non plus.»

Au début, vraiment, mon contremaître il m'a fait monter sur le machin, sur le truc, pis après, j'ai plus rien eu. Il [le patron] lui a dit à l'autre, à son collègue: " Je veux pas la voir sur une seule machine.»

On se demande, à la lecture de cet extrait, s'il s'agit d'une protection ou d'une sanction. En effet, pour éviter de nouveaux incidents, l'employeur choisit d'éloigner la jeune femme des situations potentiellement à risque, lui retirant ainsi également la possibilité de se former, puisqu'elle n'a accès ni aux pratiques (monter sur les arbres), ni aux outils (utiliser et monter

113 Cet extrait apparaît déjà au Chapitre 5 et est analysé sous l'angle de l'accès à la formation, de sanction/protection et de non-accès aux outils. Il est repris ici sous l'angle de la division sexuelle du travail. 
sur les machines). En outre, cet éloignement des outils la met également à l'écart du pouvoir que ceux-ci confèrent (Tabet, 1979). Enfin, en éloignant la jeune femme du danger, l'employeur renforce indirectement la division sexuelle du travail (Malochet, 2008), puisqu'elle ne pourra faire que certaines tâches spécifiques, pendant que ses collègues réaliseront l'ensemble des activités.

Pour signaler aux pionnières qu'elles sont des femmes avant d'être des employées, un autre élément est apparu, qui ressemble fort à la surprotection évoquée précédemment. Les pionnières dénoncent fréquemment la différence de traitement dont elles font l'objet, qui tend à les ménager mais s'avère en fait teinté de paternalisme (Dallera et Ducret, 2004). C’est ce que déplore Julie, une paysagiste de 16 ans:

Il me disait [le second employeur] que: «Y a des trucs, je laissais plus passer parce que t'es une fille, y'a des trucs j't'aurais plus engueulée, mais je l'ai pas fait parce que t'es une fille.» [...] Moi, j'suis là : «Mais si t’as quelque chose à me dire, tu me le dis et c'est tout, tu fais pas parce que je suis une fille. C'est un truc aussi j'aimerais bien que voilà, je suis une apprentie, j'veux juste être traitée comme une apprentie...»

Ce que refuse la jeune femme, c'est d'être soumise à un traitement particulier en raison de son sexe. Elle a opté pour une profession largement masculinisée et exige d'être traitée comme une personne en formation, indépendamment de son sexe.

Si dans un premier temps, tout est fait pour rappeler aux pionnières qu'elles ne sont pas des hommes (mises à l'épreuve, surprotection, traitement différencié), elles sont par la suite sans cesse renvoyées à une représentation stéréotypée de la féminité. Ainsi, celles qui ont opté pour une profession atypique dans le but de s'éloigner des normes dominantes en matière de féminité114, voient leur envie de mobilité de genre, de transgression, niée.

Ce renvoi au "féminin stéréotypé» passe tout d'abord par une division sexuelle du travail encore plus marquée que dans les situations d'insertions traditionnelles ou mixtes. En effet, en situation pionnière, la hiérarchie entre les tâches et entre les sexes apparaît clairement, tant dansl'analyse qu'en proposent les jeunes femmes, que dans la perception

114 Des études soulignent la volonté de transgression affichée par certaines pionnières. Compte tenu des informations à disposition (aucune question ne portait sur les raisons qui ont participé aux choix du métier), il est impossible de proposer une telle analyse concernant les pionnières de ce corpus. 
que les jeunes hommes ont de leurs privilèges. Les pionnières, comme les femmesinsérées dans des professions mixtes ${ }^{115}$, se plaignent d'être parfois disqualifiées sur la base de la séparation et de la hiérarchisation entre la sphère de la production et celle de la reproduction. Ainsi Alexandra, une cuisinière de 22 ans, souligne:

C'étaient des hommes qui étaient très machos [...], qui étaient très: «La femme n'a aucune place dans une cuisine et puis elle ferait mieux de rester à la maison, elle ferait le repassage, ce serait bien plus intéressant!»

Le renvoi à l'univers domestique rappelle à cette pionnière qu'elle n'est pas légitime dans le monde du travail, de surcroît dans un bastion masculin. L'exemple illustre merveilleusement le double système de valeur selon qu'un domaine relève du privé ou du public. La cuisine, lieu prototypique d'assignation des femmes dans l'univers domestique, devient ici un domaine réservé aux hommes, dès lors qu'il est professionnalisé. Autre élément intéressant, le renvoi à l'espace domestique doit donc puiser dans un autre imaginaire (celui de l'entretien du linge), puisque le renvoi à la tâche nourricière est ici impossible. Ce procédé permet aux hommes de se défendre contre l'entrée des femmes dans leur champ professionnel. Il signale également aux femmes la nature des tâches qui les définissent (entretien, ménage) comme le cadre dans lequel elles doivent évoluer (l'espace domestique).

Les pionnières sont en outre fréquemment sexualisées, et ceci de façon particulièrement stéréotypée. Elles ne sont alors pas considérées comme apprenties ou travailleuses, mais sont réduites au rang d'objets sexuels. C'est ce que relève Laetitia, une employée de commerce de 16 ans:

Il [l'autre apprenti] me regardait trop! Enfin, j'en avais un peu marre. Une fois je lui ai dit: "Arrête de me regarder quoi! [...] T'as jamais vu une meuf de ta vie?» Parce qu'il me regardait trop abusé.

Cette insistance montre à la jeune femme qu'elle n'est pas à sa place, que sa présence est surprenante. Plus généralement, ces attitudes mettent en avant l'altérité des jeunes femmes dans ces univers masculins, leur extranéité. Au cœur de l'activité professionnelle s'introduisent alors des comportements à connotation sexuelle, qui selon leur ampleur pourront

115 Les pionnières ou les femmes insérées dans un métier mixte sont confrontées de manière plus directe à la division sexuelle du travail et aux discriminations (inégalités salariales notamment). 
constituer des environnements propices au harcèlement sexuel. Julie, une paysagiste de 16 ans, qui subit plaisanteries de mauvais goût et allusions salaces, en fait l'expérience:

Alors, tu tires la gueule parce que tu travailles pas avec Marco [pseudonyme] ce matin?

$[\ldots]$

Des p'tits trucs où je pourrais répondre, parce que... " Ouais, hier j'ai vu à la télé une pub où il y avait une femme qui léchait sa glace comme ça, pis ça m’a fait penser à toi...»

[...]

Les p'tits mots de tous les jours, comme quand il [le second employeur] m'a commandé mes salopettes de travail, il me fait: «Ouais, j’vais te commander des pantalons en cuir.»

Ces remarques vont puiser dans l'univers de l'industrie pornographique et ôtent à la jeune femme son statut d'apprentie, d'employée. Elle a, dans ce cas, un statut exclusivement sexuel. Au final, c'est d'une véritable situation de harcèlement sexuel dont il s'agit:

Il [le contremaître] me fait: "On va finir cette terrasse, après j'te faisl'amour sur la table et cet après-midi, on recommence.»

$[\ldots]$

Ça lui [le contremaître] est déjà arrivé quand j’bossais, en train de faire quelque chose, ben une claque sur les fesses.

Les pionnières font doncl'expérience du coût de la transgression (Marry, 2004b, 2007) lors de mises au pas brutales et régulières. Elles subissent en fait une double violence. Les attaques usant des ressorts de la virilité (mises à l'épreuve, menaces, agressions) leur enseignent qu'elles ne sont pas des hommes, pendant que des violences spécifiques (sexisme, renvoi à la sphère domestique, discriminations) leur transmettent ce à quoi sont identifiées les femmes dans ces univers de travail. Ainsi, les jeunes femmes engagées dans une profession atypique sont confrontées à une double injonction, voire à une injonction paradoxale: être féminine pour rappeler qu'elles ont bien compris qu'elles n'étaient et ne seraient jamais des hommes, renvoyant ainsi à ces derniers l'image de l'altérité, tout en étant en mesure de se défendre contre les agressions sexistes portées à leur encontre. Pour ce faire, elles empruntent parfois 
les instruments utilisés par leurs collègues pour faire face à la souffrance, comme par exemple les stratégies de défense viriles (voir Chapitre 7): "Ainsi certaines femmes seraient amenées à jouer un rôle social de type masculin dans la sphère productive, dans la mesure où celle-ci tend à rejeter à l'extérieur ceux [ou celles] qui se différencient par trop du modèle masculin dominant» (Devreux, 1984: 124). Leur socialisation professionnelle se déroulant dans un univers masculin, réaffirmé comme tel en permanence, les pionnières apprennent indirectement qu'elles doivent adopter les attributs de la masculinité (attitudes, langage, stratégies de déni, etc.) et ce malgré les réassignations de genre (féminin) qu'elles subissent sans cesse. Elles s'approprient donc les instruments utilisés par leurs collègues masculins, mais doivent composer avec les normes de la féminité de peur de n'être plus considérées comme des femmes conformes (c'est-à-dire hétérosexuelles) (Cassell, 2000). Dans ce but, elles doivent d'autant plus user de parades de genre (Goffman, 2002) qu'elles sont en situation de transgression. Agnès, une peintre en carrosserie de 18 ans, donne l'exemple d'une de ses collègues de cours, qui a appris à gérer ces attentes paradoxales:

L'autre [pionnière de sa classe], c'est la vraie miss quoi, toujours avec les talons; elle venait aux cours avec les talons, mais la vraie miss, mais elle avait un caractère de mec, quoi, elle avait un sacré caractère...

On retrouve ici, la situation décrite par Cassell pour les chirurgiennes (Cassell, 2000) : bien que mal accueillies dans le milieu professionnel parce que femmes, elles doivent sans cesse signaler à la fois qu'elles sont compétentes (comme des hommes) et qu'elles sont bien des femmes, en mettant par exemple du rouge à lèvres, y compris lorsqu'elles sont appelées au milieu d'une garde en pleine nuit.

Que ce soit dans les métiers traditionnels ou dans les insertions atypiques, les filles sont soumises - comme les garçons - à des injonctions, qui ne sont pas dénuées d'une certaine brutalité. Si dans les milieux féminisés, la violence physique est moins fréquente, d'autres formes de brutalités sont utilisées: la moquerie, les persiflages, la violence verbale. Les jeunes femmes apprennent ainsi qu'elles doivent correspondre aux normes étroites, parfois paradoxales de la féminité. 


\subsection{Des apprentissages qui soulignent la complexité des rapports de genre}

La première partie de ce chapitre a mis en évidence le fait que la formation professionnelle est un lieu de socialisation où s'apprennent la division sexuelle du travail et la hiérarchie entre les sexes. Dans la deuxième partie, a été souligné que c'est également un lieu de transmission, de production et de renégociation de certaines normes de genre. Ces normes se forgent sur une nette séparation entre le féminin et le masculin. Cette bipartition participe de la fabrication d'une différence entre les sexes, différence qui permettra d'asseoir la division sexuelle du travail.

Paradoxalement, dans le même temps, la bi-catégorisation de sexe produit l'illusion d'une complémentarité entre les sexes. L'opposition féminité/masculinité, qui dans le monde du travail est notamment associée à des compétences spécifiques, contribue à cette illusion. Et si dans un monde du travail peu mixte, l'opposition et l'antagonisme dominent, la hiérarchie se fait plus discrète dans les autres sphères de vie. Dans ces dernières, c'est plutôt l'idéologie de la complémentarité qui règne. Celle-ci sous-tend le système hétéronormatif qui s'articule au système de genre (Jackson, 2009). En effet, la complémentarité permet la proximité entre les groupes sociaux, malgré les antagonismes. Il se fonde sur les liens existant entre les groupes sociaux, notamment les liens familiaux (Goffman, 2002), et permet in fine de maintenir le système hétéronormatif, nécessaire à la reproduction sociale (Jackson, 2009; Wittig, 2001). Ce double apprentissage de l'antagonisme et de la complémentarité fera l'objet de cette troisième partie. Cette démonstration se fera au travers d'une étude de cas, plus à même de présenter cet apprentissage. Dès lors, et compte tenu du fait que nombre d'extraits ont déjà été utilisés, ceux-ci ne seront qu'évoqués et quelques autres extraits inédits seront utilisés.

\subsubsection{L'antagonisme au travail...}

Pour illustrer le paradoxe de ce double apprentissage, l'expérience d'Agnès, la peintre en carrosserie de 18 ans, a semblé particulièrement adéquat. Non seulement son parcours nous est familier, compte tenu des nombreuses références faites tout au long de cet ouvrage, mais encore elle est sans doute l'apprentie qui expérimente le plus clairement cet apprentissage de logiques opposées ${ }^{116}$. Les différents extraits utilisés

116 Les notions de travail et de hors-travail sont utilisées par effet de contraste. Cependant, la distinction se fait plus au niveau de la temporalité que des lieux. Ainsi, l'entier de la situation décrite se déroule dans l'entreprise for- 
jusqu'ici ont surtout mis en lumière les difficultés rencontrées par la jeune femme dans ce bastion masculin.

Elle a démarré son apprentissage de peintre en carrosserie dans une petite entreprise (environ 7 employé.e.s), après y avoir effectué un stage de 2 semaines. Ses débuts dans l'entreprise, entrecoupés par la fermeture estivale, se passent bien. Mais, dès lors qu'elle démarre véritablement son apprentissage, la situation change : elle subit des remarques, se fait rabaisser. La jeune femme qualifie d'ailleurs à plusieurs reprises l'environnement de travail de « macho». Les commentaires viennent tant des tôliers que des peintres, en particulier des plus jeunes travailleurs. Le patron, quant à lui, l'a encouragée à faire ce stage et à démarrer cette formation, mais n'est que rarement à l'atelier. Elle se forme auprès d'un peintre plus âgé, qui lui ne lui fait pas de remarques désobligeantes.

L'ancienne apprentie insiste sur la question de l'ambiance et des remarques, car il est clair pour elle que ce n'est pas la non-mixité qui pose problème:

[Le problème] c'est pas parce que c'est un métier de mecs, où y avait des mecs et tout comme ça, c'était rien à voir quoi! Je pouvais me changer devant un employé, c'était pas ça qui allait me, c'est pas ça qui allait me tuer quoi, j'veux dire, c'est des employés, c'est des employés quoi. Ouais c'était vraiment leur façon d'être...

Comme le mentionnent diverses études sur les pionnières (Dallera et Ducret, 2004), ce qui retient les jeunes femmes ne relève pas des difficultés pratiques rencontrées (absence d'infrastructures ad hoc), mais du comportement des collègues. Outre les remarques sexistes, qui lui signalent qu'elle n'a rien à faire dans cet endroit, elle subit de plein fouet la division sexuelle du travail, puisqu'elle effectue principalement des tâches de nettoyage. Elle dénonce vertement cette situation: elle se sent traitée comme la femme de ménage («la conchita», «la bonne») et considère que cette assignation est directement en lien avec son sexe. Par ailleurs, cela l'empêche d'apprendre son métier, ce qu'elle constate en discutant avec ses collègues de cours. Ces derniers ont déjà acquis un certain nombre de techniques (par exemple « masquer » ou « surfacer ${ }^{117}$ )

matrice, le travail signifie les périodes où se déroule l'activité professionnelle (production, formation), alors que le hors-travail concerne les périodes intermédiaires (pauses cafés, repas, sortie de l'atelier).

117 «Masquer» signifie préparer la voiture en couvrant les surfaces ne devant pas être peintes. «Surfacer» veut dire préparer une carrosserie à la peinture en couvrant les imperfections. 
et ont eu accès aux outils (par exemple les pistolets à peinture), alors qu'elle a surtout appris à «nettoyer les voitures, nettoyer la carrosserie, nettoyer les pistolets, pis les trucs des autres». Elle comprend donc que son double statut d'infériorité (apprentie et femme dans un métier masculin) l'astreint tout d'abord à des tâches d'entretien, ensuite qu'elle est au service des autres, puisqu'elle s'occupe également de leur nettoyage, et enfin qu'elle n'accède ni aux techniques ni aux outils. Ce dernier élément rappelle la situation de la paysagiste à laquelle on avait retiré, par «mesure de précaution", l'accès aux techniques et aux outils du métier, lui retirant de fait l'accès à l'apprentissage du métier et au pouvoir que confère la maîtrise des outils (Tabet, 1979). La jeune peintre en carrosserie découvre donc que son statut de pionnière la met au service de ses collègues masculins, en ce qui concerne les tâches d'entretien et ne fait pas d'elle une professionnelle.

Par ailleurs, elle subit menaces, mises à l'épreuve physique et une certaine violence de la part de ses collègues, qui lui signalent dès qu' ils en ont l'occasion, qu'elle n'a rien à faire dans l'univers de l'industrie automobile, qu'elle a transgressé les normes de genre et qu'elle doit en payer le prix. Elle finit par quitter l'entreprise, ces mises à l'épreuve ayant eu raison de sa santé. Une blessure au genou, survenue durant une activité sportive, sera le prétexte à son arrêt. Elle précise que si le travail n'est pas à l'origine de la blessure, il l'a faite empirer, en particulier lors des mises à l'épreuve physiques.

Tous les éléments décrits précédemment sont ainsi présents dans cet exemple: la division sexuelle du travail qui permet la construction des rapports sociaux de sexe, la résistance des hommes à l'entrée d'une femme dans une profession qui fonctionne comme un bastion, enfin les pratiques masculines d'exclusion. L'antagonisme entre les groupes de sexe est particulièrement marqué ici du fait du caractère transgressif de cette insertion pionnière. En effet, en ayant opté pour une formation dans une filière atypique, la jeune femme effectue une forme de mobilité de genre (Daune-Richard et Devreux, 1992 ; Guichard-Claudic et al., 2008).

\subsubsection{La complémentarité et la complicité hors travail}

Pourtant, malgré ces relations conflictuelles, dans lesquels se construitla hiérarchie entreles sexes, une autre relation se met en place parallèlement. Elle est conviviale, repose sur la complicité, la complémentarité. Ainsi, la jeune femme découvre-t-elle des collègues qui changent d'attitude selon le moment: 
Je travaillais plus souvent avec les peintres et des fois, j'allais vers les tôliers aussi. Pour nettoyer et tout ça, pis à midi, le plus souvent, le plus vieux des tôliers en fait, le chef des tôliers, il mangeait vers moi, donc on parlait beaucoup, on discutait beaucoup... J'avais des contacts avec lui. Mais en dehors du boulot, il était super sympa, pis dès qu'on recommençait le boulot, c'était bon quoi, c'était gagné quoi, c'était un vrai connard!

Tout fonctionne comme si les relations étaient dépendantes du contexte dans lequel elles s' inscrivaient. Au travail, c'est-à-dire durant la pratique de l'activité, c'est le temps de l'opposition, de la construction de rapports de hiérarchie fondés sur des différences de statut (salarié, de genre, d'ancienneté). Hors travail, c'est-à-dire durant les pauses café, les repas et à la sortie du travail en fin de journée, c'est le temps de la complicité. Pour ces hommes, le travail est masculin, c'est même leur domaine réservé; au contraire, le hors travail est mixte. Dans ce cas précis, il est à noter que ces deux moments (travail et hors travail) interviennent dans un même espace : l'atelier. Tout semble s'organiser pour que l'apprentissage souvent brutal de rapports sociaux antagonistes ne péjore pas un autre apprentissage: celui d'un système hétéronormatif, dans lequel les relations entre femmes et hommes se doivent d'être conviviales et détendues. L'ambiguïté, ou plutôt l'alternance entre deux types de comportements, observée chez le chef tôlier se retrouve chez les autres collègues de la jeune femme:

Dès que c'étaient les pauses et tout ça, moi j'étais vers les deux plus jeunes en fait, donc on discutait, ça pouvait monstre aller; à midi, ça allait nickel, le soir quand on partait, ça allait nickel. Des fois je rentrais avec l'employé et tout, il me ramenait à la gare et tout, donc ça, ça allait nickel, mais alors pendant le boulot, mais c'était horrible!

Ces changements d'attitudes ontété expérimentés par la jeune femme dès son arrivée dans l'entreprise. Durant son stage et au cours de sa première semaine d'apprentissage, elle découvre des rapports conviviaux, puis les relations se tendent dès qu'elle décide de poursuivre sa formation:

Elles [les remarques] ont commencé, quand j’ai commencé mon apprentissage, comme par hasard.

On retrouve ici, sous un autre aspect, la question du travail (masculin) et du hors-travail (mixte). Tant que la jeune femme n'est que stagiaire, 
elle est une sorte de «curiosité » passagère. Ne cherchant pas, à ce stade, à faire partie du collectif, elle ne menace pas l'intégrité du corps professionnel et sa présence est tolérée, voire appréciée et encouragée. Pourtant, dès qu'elle devient apprentie, c'est-à-dire qu'elle cherche à s'insérer durablement dans le territoire masculin et à devenir une professionnelle, elle est perçue comme une menace (Guichard-Claudic et al., 2008; Maruani et Nicole, 1989). Elle met alors en péril l'identité de la profession et de ceux qui l'exercent (Couppié et Epiphane, 2008; Eckert, 2007; Molinier, 2000).

Il y a donc deux temporalités: celle de l'activité professionnelle et celle du hors travail. Bien que situés dans un même espace, l'entreprise ou l'atelier, ces deux moments sont radicalement opposés. Dans l'un se construisent les rapports de pouvoir entre les groupes de sexes, fondés sur la division sexuelle du travail, dans l'autre se construisent les relations interpersonnelles entre les sexes, fondées sur la norme hétérosexuelle. Ces deux moments sont cependant interdépendants. Ils illustrent les deux faces d'une même médaille: le système de genre. Ce système repose à la fois sur la division sexuelle du travail, la hiérarchie entre les sexes et sur la norme de l'hétérosexualité. Ce que l'expérience de cette pionnière fait apparaître, c'est que les apprentissages se font conjointement. Ainsi le rapport antagoniste signale-t-il que les normes de genre ne sont pas respectées, pendant que le rapport convivial rappelle la norme de l'hétérosexualité. En fait, cette jeune femme apprend que tout va bien, pour autant qu'on respecte la hiérarchie entre les sexes et les rôles assignés à chacun·e. Les comportements contrastés, entre agression et complicité, permettent de rappeler l'ordre social entre les sexes. Il faut rester à sa place, ainsi les relations interindividuelles seront détendues, sympathiques.

Ces apprentissages conjoints se font au travail, durant la formation professionnelle, mais affecteront également les autres espaces sociaux. En effet, les relations interpersonnelles se développant dans divers espaces sociaux, au travail, mais aussi dans les loisirs ou dans l'espace domestique, l'expérience vécue par cette pionnière se répercutera probablement dans d'autres sphères. Elle aura ainsi non seulement appris quelles sont les tâches quilui sont dévolues, mais encore qu' il en coûte de ne pas rester "à sa place».

Dans son article, Devreux (1992) s'intéresse en second lieu à la transversalité du genre et laisse entendre que l'apprentissage de la division sexuelle du travail et de la hiérarchie entre les sexes fait à l'armée contamine la sphère domestique, car les jeunes recrues ont appris en parallèle comment y échapper. Or, dès lors qu'ils rentrent à la maison 
(chez leurs parents ou dans leur foyer), les jeunes hommes se déchargent de ces tâches traditionnellement connotées comme féminines sur les femmes (mères, petite-amies). En formation professionnelle, on observe ce même processus du côté des hommes (apprentissage à échapper au déclassement), et une forme d'habituation à la position de subordination du côté des femmes. Les jeunes femmes découvrent qu'elles resteront dans une position de dominée, y compris lorsqu'elles auront quitté le statut d'apprentie, puisque dominées dans les rapports sociaux de sexe. Par l'alternance de relations conviviales et de remises à l'ordre brutales, les jeunes femmes apprennent à « tenir leur place», soit à s'accommoder de la subordination aux hommes. Ce n'est que par ces apprentissages conjoints que la division sexuelle du travail et la hiérarchie entre les sexes peuvent se perpétuer. Le processus a d'autant plus d'impact qu'il survient au sortir de l'adolescence pour des jeunes qui s'apprêtent à entrer dans le monde professionnel, à quitter leur famille d'origine et à forger leur propre vie de couple. On peut supposer que ce qui aura été appris durant cette socialisation professionnelle pèsera sur les pratiques ultérieures, d'autant que d'autres espaces sociaux (l'armée par exemple) viendront renforcer et confirmer ce premier apprentissage.

\subsection{L'apprentissage, une socialisation aux diverses facettes du système de genre}

Les différents mécanismes de transmission des normes de genre ont été présentés dans ce chapitre. Ils permettent de comprendre comment la formation professionnelle contribue à apprendre aux jeunes femmes et aux jeunes hommes l'existence d'un rapport dissymétrique entre les sexes, leur position réciproque dans ce rapport, les tâches auxquelles ces positions renvoient et les normes physiques et comportementales propres à ces positions particulières. Que ce soit en insertion traditionnelle ou dans les situations pionnières, ces normes s'apprennent par la confrontation au monde du travail, par les interactions avec les collègues, avec les personnes formatrices ou encore avec la clientèle. La socialisation de genre est ici renforcée par la socialisation professionnelle. Ainsi, tout en transmettant les normes d'un métier, la formation professionnelle enseigne comment être une femme ou un homme dans le monde du travail, et plus généralement dans la société.

En outre, j'aimerais souligner que cet apprentissage se fait de façon souvent brutale. On peut véritablement parler de rappels à l'ordre ou de mises au pas, tant les injonctions à la conformité peuvent être 
fortes. C'est au travers de la moquerie, de la violence verbale et parfois physique, de la menace de déclassement que ces apprentissages se font. Les jeunes apprennent donc non seulement les normes de la féminité ou de la masculinité, mais encore les risques de la transgression.

Les jeunes femmes se doivent d'être minces, élégantes, attentives à leur hygiène et avoir un certain nombre de compétences sociales (disponibilité, amabilité). Ces normes se construisent à la fois sur les stéréotypes de la féminité et sur ceux des professions dans lesquelles les femmes sont concentrées, où l'apparence et l'attention à autrui sont importants (santé, service aux personnes). Il s'agit en outre de respecter cette norme sans être associée à l'image repoussoir des femmes "vulgaires " ${ }^{118}$, que l'on retrouve comme représentation stéréotypée de la féminité dans les milieux où les femmes sont minoritaires. Les pionnières sont ainsi sans cesse renvoyées à une différence fondamentale (elles sont fragiles, minutieuses, doivent être protégées) et à une hiérarchie entre les sexes. Cette hiérarchie passe par la division sexuelle du travail ainsi que par la sexualisation des femmes, voire par une forme de mise à disposition de leurs corps pour les hommes, le sexage (Guillaumin, 1992).

Les apprentis sont eux aussi confrontés à un certain nombre d'injonctions. Soumis à un fort contrôle du collectif, ils sont incités à taire leurs peurs et leur souffrance, à mettre en avant leur goût pour la force, à banaliser la pénibilité physique. Ils doivent eux aussi avoir le "physique de l'emploi", c'est-à-dire un physique sportif (Gasparini, 2007), capable d'affronter la dureté de la tâche. Leur apprentissage de ces diverses normes se fait au travers d'une certaine rudesse, de violence et de la menace du déclassement. Celle-ci est d'autant plus efficace, qu'en apprenant la division sexuelle du travail, les jeunes hommes ont provisoirement été placés dans une position de subordination, en étant assignés à des tâches subalternes, répétitives, ennuyantes.

La marge de manœuvre semble bien mince pour ces apprenti.e.s. Occupant un statut subalterne, elles et ils sont également à un âge où transgresser les normes peut être inquiétant, dans la mesure où parallèlement à la construction d'une identité professionnelle, elles et ils construisent leur identité sexuée. Dès lors, on pourrait imaginer dans le prolongement des réflexions de Vouillot (2007), que le choix d'une profession «traditionnelle» pour son sexe comme les stratégies pour faire face aux remises à l'ordre sont au service du genre. Choisir une profession typique, c'est dire qu'on est bien conforme aux attendus

118 Autrement dit, il faut être féminine, séduisante tout en évitant le «stigmate de la putain» (Pheterson, 2001). 
sociaux en matière de genre. Se conformer aux normes attendues (tenues, comportement) peut dès lors être une stratégie de résistance aux difficultés et de maintien en formation, même si cela prend les formes de l'hypercorrection (Lamamra et Masdonati, 2008). Dans le même ordre d'idée, investir une profession atypique en jouant la partition qu'on nous assigne, à l'exemple de la collègue de cours de la pionnière citée précédemment (hyperféminine, en talons) est peut-être une stratégie pour éviter les mises à l'épreuve les plus difficiles. De même, les garçons qui jouent la virilité sur le plan physique, trouvent peut-être un moyen d'éviter une trop grande rudesse de la part du collectif de travail. Bien qu'il soit difficile de voir se profiler des dynamiques émancipatrices, on perçoit cependant dans certains récits l'existence de marges de manœuvre ou de plaisir à user de la stratégie adéquate. Il s'agirait donc de poursuivre l'analyse auprès de jeunes, ayant pu se maintenir en formation, afin de vérifier si elles et ils ont trouvé des stratégies pour composer, voire jouer avec ces normes. 


\section{Conclusion}

Travailler sur la formation professionnelle dans une perspective de genre offre une occasion unique d'aborder un champ peu exploré de l'analyse scientifique. La formation professionnelle se situant à l'intersection entre école et marché du travail, cette recherche a permis de faire apparaître les effets de la socialisation acquise durant la scolarité, de voir en œuvre les logiques de ségrégation du marché du travail et, enfin, de cerner les contours des normes de genre et de comprendre leur mode de transmission. Plus spécifiquement, ces réflexions ont mis en lumière l'organisation du travail en formation professionnelle. Celle-ci repose notamment sur la division sexuelle du travail et sur le statut d'apprenti.e, ce qui a été analysé à travers la délégation des tâches subalternes et d'entretien aux apprenti.e.s.

L'analyse secondaire de données issues d'un corpus déjà étudié offre ainsi une opportunité de traquer le genre dans les expériences du quotidien. Les nombreux éléments mis en évidence par l'analyse soulignent la force du système de genre, sa permanence et sa transversalité. En effet, même lorsqu'il ne constitue pas l'objet central de la recherche, il se donne à voir systématiquement. Il apparaît dans les processus dans lesquels les personnes sont engagées et qui peuvent, comme ici, les conduire à interrompre leur formation. Le genre agit également sur les interactions entre apprenti.e.s et collègues ou employeur.e.s. Enfin, il se joue dans la relation qu'elles et ils entretiennent avec les chercheur.e.s.

La première question de recherche a mis l'accent sur la différence. Il importait en effet de déterminer si les processus qui conduisent les jeunes à interrompre leur formation varient entre filles et garçons. Mes résultats sont contrastés: de manière globale, le processus semble identique (raisons expliquantl'arrêt, souffrances vécues, stratégies déployées), mais l'expérience est imprégnée par le genre. En effet, le contexte général du marché du travail et de l'apprentissage, la ségrégation et la socialisation secondaire, concourent à marquer différemment les expériences vécues par les filles et les garçons. Inséré.e.s dans des professions sexuées, organisées autour de normes de genre, les difficultés ne sont pas semblables pour les unes et les autres. Effet de la socialisation primaire et secondaire, filles et garçons se positionnent face à la formation professionnelle et plus généralement face à l'emploi de façon différente. Ces éléments se com- 
binent pour proposer, in fine, des situations relativement dissemblables. D'un côté, des filles qui peinent à s'identifier à l'emploi et se centrent davantage sur l'accès à la formation, qui évoquent plus fréquemment leur situation et les relations de travail en termes de relations interpersonnelles, et qui parlent ouvertement et avec précision de leur souffrance. Elles subissent en outre de plein fouet les discriminations et les violences liées à leur sexe, tout particulièrement lorsqu'elles ont transgressé les normes de genre en choisissant une profession atypique. De l'autre, des garçons qui anticipent leur futur rôle de pourvoyeur principal, en se centrant sur l'emploi, qui n'évoquent que rarement leur souffrance et font preuve de virilité en subissant en silence. Ils se positionnent très vite dans un rapport salarial et dénoncent leur situation en mettant en avant le non-respect du contrat (tacite ou formel). Enfin, ils déclinent la question relationnelle en termes d'ambiance et de climat de travail, et non en termes interpersonnels.

L'analyse comparée mobilisée pour répondre à la première question de recherche, a conduit à examiner la manière dont les personnes doivent gérer les contraintes (organisationnelles, de genre, etc.) dans leur quotidien au travail. L'analyse de contenu thématique a permis d'accéder à ces informations et à comprendre la «façon dont les gens apprennent, acceptent, négocient ou récusent les catégories de 〈différence> qui modèlent la vie quotidienne» (Ginsburg et Tsing citées par Cassell, 2000: 63). La question n'est plus tant de savoir si l'expérience en formation professionnelle des apprenties est différente de celle des apprentis, mais de comprendre comment chacun.e négocie avec les normes en jeu dans l'univers professionnel et dans les interactions avec les collègues et les supérieur.e.s.

La deuxième question de recherche a plus été dans le sens d'une analyse de la formation professionnelle comme lieu de perpétuation du système de genre, et, simultanément, de production de normes nouvelles. On peut véritablement parler ici de «fabrication du genre» (Cassell, 2000; West et Zimmerman, 2009). Il s'agit pour les personnes en formation d'apprendre à être un.e professionnel-le sexué.e, sur un modèle qui peut varier selon le secteur d'activité. Les normes de genre se mêlent alors étroitement aux normes professionnelles et leur transmission se fait au cours de la socialisation professionnelle, durant laquelle les jeunes sont poussé.e.s, encouragé.e.s, forcé.e.s à adopter un comportement jugé adapté à leur sexe.

L'expérience en formation professionnelle va au-delà dela transmission et del'intégration de ces normes, elle donne également à comprendre 
la clef de voûte du système de genre: la division sexuelle du travail. Pour ces jeunes à peine sorti.e.s de la scolarité obligatoire, bien qu'elles et ils aient auparavant subi ou bénéficié de cette forme de division du travail (par exemple au travers de leur participation plus ou moins importante aux tâches domestiques), c'est une véritable découverte. Tout d'abord, ces adolescent.e.s débutent une formation dans un contexte peu, voire non mixte. Ensuite, elles et ils expérimentent en tant qu'apprenti.e.s une organisation du travail fondée sur la division sexuelle du travail: au bas de la hiérarchie, les apprenti-e.s effectuent les tâches dévalorisées traditionnellement assignées aux femmes (rangement, entretien). La formation professionnelle est donc le lieu de l'apprentissage de diverses hiérarchies (notamment, mais pas seulement, celle liée au sexe). Ce faisant, filles et garçons apprennent leur position respective dans ces rapports sociaux inégaux.

Parallèlement, un second apprentissage intervient. Il porte sur un autre élément central du système de genre: l'hétéronormativité. Elle s'impose notamment par des normes professionnelles qui sexualisent le travail (codes d'habillement, attente d'adéquation à certaines normes physiques, etc.). Ces normes s'appuient sur celles de la féminité et de la masculinité, tout comme sur celles de la séduction. Ainsi, avoir «le physique de l'emploi » sous-entend entrer dans les codes de la séduction hétérosexuelle, et devient une spécification personnelle dans certains métiers (Jackson, 2009). Toujours présente, la sexualisation du travail fait partie de ce qui est socialement admis. Elle prend cependant des formes plus violentes lors de tensions et de conflits: attitudes et propos sexistes, recours à la pornographie, harcèlement sexuel. Dans les situations de ce type, la sexualisation du travail fait apparaître des rapports entre les sexes fondés tant sur la suprématie du masculin sur le féminin que sur l'hétéronormativité.

La formation professionnelle apparait donc bel et bien comme une «fabrique du genre», puisque au cours de l'expérience dans cette filière, différents apprentissages se font conjointement: la conformité à des normes de féminité/masculinité, la soumission à la hiérarchie entre les groupes de sexe et au système hétéronormatif. Durant la formation professionnelle, les jeunes expérimentent les fondements du système de genre, en apprenant qu'elles et ils occupent des positions antagonistes dans les rapports sociaux de sexe, tout en baignant dans l'idéologie de la complémentarité soutenant l'hétéronormativité.

Au centre de l'analyse de la formation professionnelle comme lieu de production du genre figure l'hypothèse suivante: certains arrêts de 
formation peuvent être renvoyés à la transmission des normes de genre. Plus exactement, l'arrêt peut être envisagé, dans certains cas, comme une réponse à la souffrance provoquée par la contrainte à la norme de féminité ou de masculinité. Filles et garçons subissent en effet de fortes injonctions à la conformité de genre: les garçons doivent faire preuve de virilité, soit de force, d'endurance, de résistance à la peur et à la douleur. Les filles se doivent d'être féminines, soit faire attention à leur apparence tant corporelle que vestimentaire, être «séduisantes» et attentives à autrui. Les éléments issus de l'analyse secondaire de contenu thématique amènent un certain nombre d'arguments qui vont dans le sens de cette hypothèse.

En premier lieu, ce qui pose problème n'est pas tant l'inadéquation aux normes de genre dominantes dans une profession ou un secteur d'activité, que la brutalité de l'injonction à la conformité et la violence de leur transmission. Les mises au pas et les rappels à l'ordre se font par la moquerie, le dénigrement, l'humiliation, la mise à l'épreuve physique. La violence de la transmission des normes de genre est attestée d'une part par l'importante souffrance mise en évidence dans les analyses, et, d'autre part, par la prégnance des questions relationnelles dans les raisons d'arrêt invoquées. Ainsi, une personne n'interrompt pas parce qu'elle ne serait pas assez «féminine » selon les critères d'une profession donnée ou pas assez "virile» selon les critères d'une autre, mais parce que l'assignation à être féminine ou viril est brutale. Ces contraintes sont parfois directes et explicites, d'autres fois larvées et sournoises, en particulier lorsque les reproches portent sur de soi-disant incapacités professionnelles.

Le deuxième élément qui va dans le sens de cette hypothèse est le statut d'apprenti.e. L'expérience de cette position subalterne, accentuée par l'assignation à des tâches connotées comme féminines, peut-être, dans certains cas, insupportable (Renard, 2015). Du point de vue du genre, elle l'est surtout pour les garçons. Bien que leur assignation au travail domestique ne soit que temporaire, elle les place dans une position qui entre en contradiction avec celle qu' ils occupent habituellement dans les rapports sociaux de sexe. On peut dès lors postuler que certains garçons arrêteraient à cause d'un sentiment de déclassement. Les personnes ayant envisagé la formation professionnelle comme "réparatrice», suite à un parcours scolaire chaotique, peuvent ressentir ce déclassement de manière encore plus aiguë. L’assignation à des tâches dévalorisées, socialement définies comme féminines, apparaît alors intolérable. 
Troisièmement, le lien entre arrêt de formation et normes de genre apparaît de manière particulièrement explicite pour les personnes ayant choisi des professions atypiques. L'injonction à la conformité concerne plus précisément les pionnières, sans cesse renvoyées à leur altérité dans un univers de travail masculin. Elles doivent faire face à une féminité stéréotypée (pornographique) et paient cher la transgression qu'elles ont commise en franchissant les frontières de genre (attitudes et propos sexistes, mises à l'épreuve, harcèlement sexuel, etc.). Les pionniers quant à eux découvrent la contradiction dans laquelle les place leur choix atypique. Insérés dans des professions dévalorisées, mal rémunérées, avec de faibles perspectives de carrière, ils quittent ces domaines. Le métier ne permet ni reconnaissance sociale, ni ressources économiques assurant leur futur rôle de pourvoyeur principal.

Ainsi, le genre participe bien du processus de l'arrêt, il intervient à différents niveaux. Pour autant, il serait erroné d'affirmer queles normes de genre, la violence de leur transmission sont la cause de l'arrêt.

Dans certaines situations, comme en cas d'insertion pionnière ${ }^{119}$, la transmission des normes de genre participe de l'arrêt, mais ce n'est pas systématique, puisque certain.e.s font carrière. Il s'agit alors de réfléchir aux conditions qui font que les un.e.s partent et les autres restent. Certains arrêts sont également les réponses à la mobilité descendante opérée au cours de la formation professionnelle, des garçons disant ainsi leur refus du déclassement qu'ils subissent du fait de leur statut d'apprenti. Pour autant, il est impossible d'affirmer que les personnes qui arrêtent signalent nécessairement leur refus de l'assignation à des normes de genre contraignantes, laissant entendre que les personnes qui se maintiennent en formation se conformeraient sans autre à celles-ci.

Enfin, établir un lien de causalité entre normes de genre et arrêt prématuré en formation professionnelle reviendrait à dire que celles-ci n'interviendraient pas dans l'expérience des apprenti.e.s qui n'interrompent pas leur formation. Or, comme les raisons ou la souffrance l'ont montré, les expériences sont marquées par le genre, car les contextes professionnels sont sexués. L'expérience en formation professionnelle est organisée par la division sexuelle du travail, par la ségrégation. Il semble dès lors évident que ces éléments sont également présents dans l'expérience vécue par celles et ceux qui n’arrêtent pas. Il serait intéressant

119 Une étude allemande souligne que les pionnières ont des risques accrus d'interrompre prématurément leur formation (Rédaction, 2008). Leur taux d'arrêt est plus important que celui des pionniers, et que celui des jeunes en insertion traditionnelle. 
de poursuivre la recherche du côté des personnes qui se maintiennent en formation, en questionnant les stratégies mises en œuvre pour faire face aux difficultés rencontrées, notamment les parades de genre, mais aussi en s'intéressant au réseau et aux soutiens que ces personnes ont à leur disposition. Finalement, si le genre contribue à certains arrêts, il participe également à certains maintiens en formation, et ce malgré la violence de la transmission des normes de genre observées tout au long de ce travail.

Cependant, une question n'a été qu'effleurée au cours de l'analyse: à l'encontre de quoi s'exerce cette violence? Il ressort des analyses qu'elle s'exerce principalement à l'end roit du corps, contre lui. Ce retour du corps au centre de l'attention, rappelle que le travail suppose une mobilisation physique, cognitive et subjective (Dejours, 1998; Filliettaz et al., 2008; G. Moreau, 2000; Renard, 2015). L'apprentissage du travail se faisant au travers du corps, l'apprentissage du genre au travail se fait de la même manière (Lamamra, 2014) : «il y a [dans la négociation des normes de genre, dans le déploiement du genre] quelque chose d'autre en jeu, qui échappe à la logique et au verbe, profondément inscrit dans les corps » (Cassell, 2000: 71). Le corps est ainsi mobilisé de différentes manières, que l'on peut synthétiser comme suit: le corps conforme; le corps sexualisé; le corps comme incarnation des pratiques d'exclusion; les limites du corps et enfin le corps limité.

Premièrement, il y a la conformité physique attendue dans nombre de professions. Il faut en effet avoir le «physique de l'emploi» (Gasparini, 2007; Hidri, 2005), qui diffère selon le secteur d'activité et le sexe du métier. Lorsque le corps ne correspond pas, il faut le transformer, le construire, l'endurcir (Hidri, 2005). Les indications sont clairement établies pour les deux catégories de sexe. Les filles doivent répondre aux critères de minceur, beauté et attention à soi (tant en termes d'élégance vestimentaire que d'hygiène corporelle), soit avoir un corps sexué, qui peut «séduire sans laisser le corps sexuel s'imposer» (Moulin, 2005). Lorsqu'elles y dérogent, elles sont rapidement remises au pas, comme cette pionnière qui se voit reprocher de «jouer avec son physique» car elle ose bouger son corps librement, sans respecter la position de retrait, voire de soumission qu'on lui impose. Les garçons quant à eux doivent faire preuve de force, d'endurance, de résistance, soit, même si cela n'est pas clairement exprimé, développer un corps d'athlète ou, pour reprendre les termes de Cassell (2000) avoir «l'étoffe des héros » ${ }^{120}$. Eux aussi sont

120 Cette expression a surtout été retenue pour des professions telles que chirurgien ou pilote, elle peut cependant s'adapter au contexte de la forma- 
rappelés à l'ordre lorsqu'ils ne correspondent pas aux attendus, ils sont raillés, traités de «femmelettes" lorsqu'ils n'ont pas suffisamment de force pour porter les sacs de ciment ou d'herbe coupée.

Deuxièmement, l'activité met en scène un corps sexualisé. Les rapports de domination exercés sur les apprenti.e.s s'incarnent dans la façon dont elles et ils sont sexualisé.e.s. La virilité comme stratégie défensive au travail se mêle d'une connotation érotique (Dejours, 2000b), ceux qui «ont l'étoffe des héros se perçoivent comme les détenteurs du phallus» (Cassell, 2000: 55). Aussi, lorsque l'on veut remettre en cause un homme, est-ce sa virilité qui est attaquée, ses capacités sexuelles qui sont mises en doute, ses attributs sexuels qui font l'objet de moqueries. Lorsque la virilité est raillée, ce n'est pas le courage ou la force qui sont questionnés, mais le corps sexualisé. Parallèlement, lorsque dans les mêmes environnements professionnels, la féminité - celle-là même à l'encontre de laquelle s'est construite la virilité - est caricaturée, c'est à nouveau le corps sexualisé qui est mis en scène. Réduit à une image pornographique, le corps est mis à disposition, offert aux hommes présents. C'est donc au travers du corps tant féminin que masculin que la hiérarchie s'affirme, que la domination se perpétue (Molinier et Grenier-Pezé, 2000).

En troisième lieu, le corps est mobilisé dans le cadre de pratiques d'exclusion. Il ne s'agit plus ici de signaler à une personne qu'elle n'est pas conforme aux attendus de la profession, mais qu'elle n'y a pas sa place. Ces pratiques d'exclusion mobilisent le corps, le mettent à l'épreuve, le violentent. C'est en blessant de manière répétée le corps d'une pionnière (en la mettant à genoux) qu'on lui dit qu'elle n'a rien à faire dans un garage; c'est en la faisant tomber d'un arbre, au risque de la blesser gravement, qu'on signale à une autre jeune femme qu'elle n'est pas la bienvenue dans les métiers verts. Enfin, c'est au travers de la violence physique qui règne dans les cuisines - qui se transforment en une véritable arène, un ring, où les collègues s'empoignent et s'affrontent dans de vrais corps à corps - qu'on fait comprendre à une cuisinière qu'elle n'est pas à sa place.

tion professionnelle à des professions moins prestigieuses, mais exposant les personnes à des risques importants. Par exemple, dans les métiers du bâtiment, les ferblantiers/ferblantières ou les charpentiers/charpentières bravent le vertige en se tenant sur les toits et font preuve d'endurance pour affronter les conditions climatiques d'une activité en plein air. Dans un autre secteur, l'industrie chimique, les employé.e.s s'exposent également à des risques en manipulant certains composants toxiques. 
En quatrième lieu, l'arrêt laisse parfois entrevoir les limites du corps, à l'instar de cette paysagiste qui pleure tous les soirs - tant la douleur et la fatigue sont fortes - et qui doit se résigner à abandonner un métier qui lui plaît. Ou encore ces jeunes hommes épuisés, éreintés par les charges qu'ils doivent porter dans les échafaudages, et qui ne parviennent pas à imaginer devoir affronter la pénibilité durant des années. Ou enfin, cet apprenti qui essaie de surmonter la peur des machines inscrite dans son corps suite à des accidents du travail. En tremblant devant les machines, en s'immobilisant face à elles, le corps met en péril la stratégie virile de dépassement de la peur. L'entier du collectif de travail soutient l'effort, car sans cela, c'est tout le système de défense qui s'effondre, la vulnérabilité et la souffrance se donnant soudain à voir.

Enfin, dans d'autres cas de figure, l'arrêt est à mettre en lien avec les limites que les normes de genre imposent aux corps, en cherchant à les discipliner. Cette situation s'est présentée à deux reprises dans le corpus. Il s'agit de deux cas qui avaient été classés dans les "choix par défaut», et où les jeunes femmes avaient été orientées (l'une par ses parents) ou avaient choisi un métier conforme à leur sexe: employée de commerce. Or, toutes deux souhaitaient «bouger», être à l'extérieur et se retrouvaient dans des professions de bureau, leurs corps disciplinés, façonnés par les contraintes d'une activité sédentaire. Ces professions s'inscrivent en effet dans le prolongement de l'éducation des filles, contrariées dans leurs envies de motricité et invitées à rester tranquilles et à se tenir bien (Messant-Laurent, 1990).

Ces deux derniers points mettent en lumière des problématiques spécifiques à certains champs professionnels: les «limites du corps» caractérisent plutôt les professions masculinisées, tandis que la question du «corps limité» se pose avant tout dans des professions féminisées.

La présence du corps est d'autant plus remarquable que parallèlement à cela la formation professionnelle, par ses évolutions récentes, connaît une élévation de son niveau d'exigence, qui peut être qualifiée d'intellectualisation. Il y a donc une tension qu'il s'agirait d'explorer entre d'une part, des professions qui s'intellectualisent, et, d'autre part, des transmissions de savoirs, mais aussi de normes qui continuent à passer par le corps.

Pour rappel, la recherche sur laquelle repose cette étude avait pour objectif de comprendre le processus menant à un arrêt de formation et n'intégrait pas, initialement, de perspective de genre. Dès lors, certaines données sont lacunaires, celles concernant les raisons sous-tendant les choix professionnels, dont les choix atypiques, ou celles prenant en 
compte d'autres rapports de domination. Des analyses complémentaires permettraient d'approfondir la question des politiques d'égalité dans la formation professionnelle, de l'insertion des jeunes dans un marché sexué et de la formation professionnelle comme institution productrice de genre. Ces limites, et les perspectives qui en découlent, vont être déclinées en six points.

Premièrement, lors des entretiens, les chercheur.e.s n'ont pas investigué de manière systématique les raisons concourant à un choix d'orientation professionnelle. L'accent portait davantage sur la manière dont le métier ou l'entreprise avait été sélectionnée que sur la raison de ce choix. Cela également en ce qui concerne les choix pionniers. Une information systématique à ce propos aurait permis d'analyser plus finement certaines motivations, notamment lors de choix atypique (par exemple, l'envie de transgression).

Deuxièmement, l'analyse a privilégié les rapports de genre et a peu exploré d'autres rapports de domination (Kergoat et Capdevielle-Mougnibas, 2015). Si la hiérarchie rencontrée dans le cadre de la formation professionnelle repose principalement sur la division sexuelle du travail, elle n'en est pourtant pas moins une relation entre patron'ne et apprenti.e, qui peut parfois prendre la forme d'un rapport d'exploitation. Il serait alors intéressant de compléter l'analyse de genre par une approche menée également en termes de rapports de classe et de "race», procédant ainsi à une analyse de l'articulation de ces différentes formes de domination (Kergoat, 2015), qui permettrait de comprendre dans quelles tensions et contradictions se trouvent certain.e.s apprenti.e.s. Deux exemples viennent illustrer l'intérêt d'une prise en compte de divers rapports sociaux. D'une part, le cas d'une jeune femme somalienne travaillant en situation pionnière dans un environnement exclusivement masculin est particulièrement intéressant. Sa position dans la fratrie (aînée) et son statut familial (elle est la première à avoir le droit de travailler en Suisse) la met dans une situation doublement pionnière, car on attend d'elle qu'elle ouvre l'accès au travail et à la formation à ses frères et sœurs plus jeunes. Elle a ainsi une forme de parcours émancipateur, mais par ailleurs a la charge, dans sa famille, de s'occuper de ses frères et sœurs durant son "temps libre». Elle n'a donc pas les occupations de bien d'autres jeunes de son âge et endosse une part du travail de $\operatorname{care}^{121}$. Cette assignation la

121 Le terme anglais est utilisé ici pour parler des tâches de soins à autrui auxquelles se mêle le souci d'autrui. Ces activités concernent de nombreux domaines (la prise en charge des enfants, le ménage, les soins à domicile ou en hôpital, le travail des assistant.e.s sociales et sociaux, etc.). Il s'agit 
renvoie à son statut de femme. D'autre part, l'exemple d'un jeune homme issu d'un milieu ouvrier est lui aussi à relever. Son père travaille dans un métier du bâtiment, secteur pour lequel il opte lui aussi, alors que la filière suivie au secondaire lui aurait permis d'accéder au gymnase (lycée). Il adopte à l'égard du monde du travail, de la hiérarchie et plus généralement du secteur du bâtiment, une attitude très critique. On peut même dire qu'il refuse complètement la socialisation professionnelle qu'il assimile à un formatage. Ce jeune homme est ainsi pris dans des logiques de reproduction sociale et de crainte de trahir sa classe, tout en rêvant de quitter la condition ouvrière (P. Kergoat, 2006; Terrail, 1990).

Troisièmement, la présentation du contexte mériterait d'intégrer une analyse détaillée des politiques de promotion de l'égalité en formation professionnelle et en particulier des politiques d'encouragement aux parcours pionniers. Cet éclairage mettrait en évidence les tensions entre, d'une part, des politiques incitatives mises en œuvre au niveau fédéral, et, d'autre part, des logiques de repli identitaire intervenant au niveau des secteurs professionnels, voire des entreprises.

En quatrième lieu, il aurait été utile que la recherche initiale porte davantage l'attention sur les secteurs d'activité, leur degré de mixité, leurs contraintes particulières. Ces éléments contextuels ont pu être reconstitués a posteriori, mais l'analyse aurait été plus fine s'ils avaient constitué un véritable arrière-plan des entretiens. Cela aurait notamment permis d'approfondir les problèmes rencontrés par les apprenti.e.s, par exemple en cernant davantage d'indices de l'hostilité des hommes à l'égard des pionnières (entre autres, posters et images pornographiques dans l'environnement de travail). Comme les filles n'ont pas directement évoqué ces images, les entretiens n'abordent pas la question. C'est donc sur la base de leur silence qu'il est supposé que les pionnières, voire d'autres filles, développent une stratégie de banalisation (Dallera et Ducret, 2004; Pfister Giauque et Flamigni, 2010) ou de déni pour neutraliser la violence qui leur est adressée. Des données plus précises à ce sujet permettraient d'étayer les résultats de ces analyses secondaires sur le lien entre arrêts prématurés et normes de genre.

Cinquièmement, toujours en ce qui concerne le contexte de la formation professionnelle, il serait intéressant dans une prochaine recherche d'investiguer ce que l'institution (notamment l'école professionnelle) produit comme normes de genre. Une analyse intégrant les contenus

de souligner que «les femmes [...], de par leur position dans le système de genre, [sont] les pourvoyeuses principales de care dans la famille et dans les métiers de soin et d'aide» (Modak, de Kinkelin et Benelli, 2008). 
des enseignements pratiques et théoriques mériterait d'être menée. Cela permettrait de réaliser pour la formation professionnelle, tant pour le volet école que pour le volet entreprise, une analyse transversale telle que l'a menée Mosconi pour l'école obligatoire (Mosconi, 1994).

Enfin, le recours à un seul outil de collecte de données (l'entretien) n'a pas permis d'analyser l'ensemble des stratégies mises en œuvre (par exemple, la banalisation évoquée ci-dessus). Des observations dans l'entreprise formatrice complèteraient les données et feraient sans doute émerger d'autres configurations de stratégies. Par ailleurs, l'observation permettrait certainement d'examiner les interactions entre apprenti.e et personne formatrice, donnant ainsi accès aux modes de transmission non seulement des normes de genre, mais aussi des savoirs pratiques. Ce serait alors l'occasion de voir en œuvre les moments où le corps est mobilisé, façonné, discipliné.

Il ressort des résultats que la psychodynamique du travail pourrait donner lieu à d'autres analyses en formation professionnelle. L'étude des stratégies utilisées pourrait par exemple être menée également auprès des personnes qui se maintiennent en formation ${ }^{122}$. L'analyse de genre permettrait en outre de savoir si ces personnes ont adopté les normes dominantes, si elles ont trouvé une marge de manœuvre ou encore si elles ont subverti ces normes, dans le sens de la "féminité comme mascarade» (Molinier, 2002b). Ce prolongement de l'analyse menée au travers de la psychodynamique du travail permettrait de souligner la transversalité du système de genre. Celui-ci intervient d'une part dans le processus conduisant à l'arrêt, l'interruption fonctionnant alors comme une stratégie défensive permettant de s'émanciper des normes de genre. Et d'autre part, d'autres stratégies composent avec ces normes de genre (jouer la conformité, incarner le rôle assigné, etc.), afin de se maintenir en formation. Dans ce cas, le genre intervient dans le maintien.

De manière générale, la formation professionnelle duale ou à plein temps gagnerait à être analysée avec les outils de la psychodynamique du travail. Dans un pays où la formation professionnelle, notamment sous sa forme duale, est largement plébiscitée, il serait indispensable de réfléchir aux contraintes spécifiques de cette voie de formation, pour l'heure trop peu étudiées. Analyser par exemple la tension entre produire et apprendre permettrait d'aborder de manière inédite la question des

122 Une première analyse de ce type a été menée dans le cadre d'un mémoire de master à l'Université de Lausanne par Géraldine Bétrisey, et fait apparaître que la stratégie privilégiée par les jeunes qui se sont maintenus en formation est l'endurance. 
transitions école-travail et, plus généralement, celle de l'insertion professionnelle. Les difficultés de transition pourraient être lues comme des difficultés à gérer cette tension, ou comme un refus par les apprenti.e.s de l'un ou l'autre de ces deux aspects. Certain.e-s jeunes désirant davantage être considéré.e.s comme des professionnel.le·s et être plus impliqué.e.s dans la production, pendant que d'autres souhaiteraient continuer à être considéré.e.s comme des personnes en apprentissage.

Un des éléments central de l'analyse est le statut d'apprenti·e, qui incarneà lui seul nombre de rapports de domination (qualification, statut, âge) présents durant la formation professionnelle, mais également tout au long de l'expérience sociale du travail. Partir de ce statut hybride et ambigu permet, cet ouvrage en donne un aperçu, de proposer une analyse originale de l'organisation et de la division du travail. En examinant ce statut au fur et à mesure du déroulement de la formation professionnelle, il serait possible de cerner la façon dont les individus prennent ou non activement part à la division du travail. Si en première année, les apprenti.e.s n'ont aucune marge de manœuvre, elles et ils apprennent qu'il leur suffit d'être en deuxième année pour pouvoir se décharger du «sale boulot» sur des personnes plus jeunes.

Enfin, il serait également intéressant d'investiguer plus avant la question de la mobilité de genre ou plutôt des différentes formes de mobilité de genre. Cette notion a fréquemment été utilisée pour qualifier le parcours des pionnières, qui transgressent les frontières du genre et effectuent de fait une forme de mobilité ascendante. Il s'agirait alors de réfléchir à d'autres formes de mobilité de genre, en particulier celle vécue par les pionniers lorsqu'ils s'insèrent dans des professions socialement dévalorisées. Dans leur cas, il s'agit plutôt de mobilité descendante. Si les apprenti.e.s partagent le même vécu et un certain sentiment de déclassement, ce dernier concerne surtout les garçons, que leur statut cantonne à des tâches subalternes connotées comme féminines. Dans leur cas, on pourrait parler de mobilité involontaire. La mobilité de genre a surtout été abordée en termes de changement de position, la mobilité ascendante signalant par exemple des filles intégrant une situation traditionnellement occupée par des garçons. L’analyse de ce processus pourrait être approfondie par l'étude de l'adoption de normes de genre traditionnellement associées aux personnes d'une autre catégorie de sexe. Il s'agit par exemple des garçons utilisant des stratégies de muliérité, lorsqu'ils sont en situation dominée, ou des pionnières recourant à des stratégies viriles. Le recours à des normes non habituelles pour son sexe pourrait également être qualifié de mobilité. L’analyse détaillée 
de ces parcours de mobilité - ascendante, descendante, volontaire ou involontaire - permettrait d'enrichir la réflexion sur le lien entre arrêt de formation et normes de genre et ouvrirait de nouvelles perspectives de genre sur l'analyse des parcours. 


\section{Références bibliographiques}

Alderson, M. (2004). La psychodynamique du travail: Objet, considérations épistémologiques, concepts et prémisses théoriques. Santé mentale au Québec, 29, 243-260.

Allen, J. P., Philliber, S., Herrling, S. et Kuperminc, G. P. (1997). Preventing Teen Pregnancy and Academic Failure : Experimental Evaluation of a Developmentally Based Approach. ChildDevelopment, 64(4), 729-742.

Alonzo, P. (2005). Trois entreprises face à l'intégration des femmes dans les emplois industriels, le cas de Saint-Nazaire. Cahiers Pierre Léon, 6.

Alvarez, E. (2005). Le genre au regard de la statistique. Paper presented at the Work in Progress du LIEGE, Université de Lausanne.

Alvarez, E., Praz, A.-F., Hertz, E., Lachat, S., Bachmann, L., Rochat, S. et al. (Eds.).(2007). Nouvelles Questions Féministes. Perspectivesféministes en sciences économiques, 26(2). Lausanne: Antipodes.

Amos, J. (2007). Transitions école-emploi : apports de la recherche TREE. In $\mathrm{M}$. Behrens (Ed.), La transition de l'école à la vie active ou le constat d'une problématique majeure (pp. 43-53). Neuchâtel: Institut de recherche et de documentation pédagogique (IRDP).

Andro, A., Bachmann, L., Bajos, N. et Hamel, C. (2010). La sexualité des femmes: le plaisir contraint. Nouvelles Questions Féministes, 29(3), 4-13.

Angelini, C. et Esman-Tuccella, S. (2004). Collège en zep: quand la parole des élèves fait écho à l'ambiguïté du travail infirmier. Travailler, 1(11), 123-146.

Arnett, J. J. (2000). Emerging Adulthood. A Theory of Development From the Late Teens Through the Twenties. American Psychologist, 55(5), 469-480.

ASCAPA.(1920). Orientation professionnelle. Conférence du 24-25.09.1920à l'Université de Genève. Genève:Meyer/Association Suisse de Conseils d'Apprentissage et de Protection des Apprentis.

Bahl, A. (2012). Ausbildendes Personal in der betrieblichen Bildung: Empirische Befunde und strukturelle Fragen zur Kompetenzentwicklung. Bonn: Bundesinstitut für Berufsbildung.

Bardin,L. (1986). L'analyse de contenu. Paris:Presses Universitaires de France.

Baudelot, C. et Establet, R. (1992a). Allez les filles! Paris: Le Seuil.

Baudelot, C. et Establet, R. (1992b). Enseignement professionnel: une mixité difficile. In C. Baudelot et R. Establet (Eds.), Allez les filles! (pp. 159-185). Paris: Le Seuil. 
Baudelot, C. et Establet, R. (1992c). Sexe et origine sociale: deux régimes distincts d'inégalité. In C. Baudelot et R. Establet (Eds.), Allez les filles! (pp. 141-158). Paris: Le Seuil.

Baudelot, C. et Leclerq, F. (2005). Egalités: la promotion des femmes. In C. Baudelot et F. Leclerq (Eds.), Les effets de l'éducation (pp. 181-183). Paris: La Documentation française.

Baudoux, C. et Noircent, A. (1997). L'école et le curriculum caché. In C. Laure-Gaudreault(Ed.),Femmes, éducationet transformationssociales (pp. 105-128). Montréal: Les éditions du remue-ménage.

Baumeler, C., Lamamra, N. et J. Schweri (2014). Les formateurs et formatrices en entreprise, personnes clefs de la socialisation professionnelle. Projet FNS n 100017_153323.

Benelli, N., Rosende, M. et Messant-Laurent, F. (2008). Dynamiques actuelles du travail dans le contexte néofordiste en Suisse: le cas de la coiffure et $d u$ guichet. Rapport de recherche, projet FNS nº 100012-111961/1: Université de Lausanne.

Bertschy, K., Böni, E. et Meyer, T. (2007). An der zweiten Schwelle: Junge Menschenim Übergangzwischen Ausbildungund Arbeitsmarkt.Ergebnisübersicht desJugendlängsschnitts TREE, Update 2007. Bern :TREE.

Billett, S. (2002). Workplace Pedagogic Practices: Co-participation and Learning. British Journal of Educational Studies, 50(4), 457-481.

Bois, P. (1980). L'Union syndicale suisse et le droit du travail. In USS (Ed.), Un siècle d'Union syndicale suisse 1880-1980 (pp. 79-102). Fribourg: Office du Livre / Union syndicale suisse.

Boivin, M.-D. et Fournier, G. (2006, Juillet 2006). L'expression des nouvelles temporalités chez des jeunes en processus d'insertion. Paper presented at the AIPTLF, Hammamet.

Bonoli, L. (2011, 17.03). La formation professionnelle entre progressisme et conservatisme, entre instruction technique et éducation globale du citoyen. Les arguments à l'origine du système suisse de formation professionnelle. Paper presented at the Rencontre interne de l'Axe de recherche 3, IFFP, Zollikofen.

Borkowsky, A. (2001). Women and Men in Swiss vocational education. In P. Gonon, K. Haefeli, A. Heikkinen et I. Ludwig (Eds.), Gender Perspectives on Vocational Education. Historical, Cultural and Policy Aspects (pp. 19-34). Bern: Peter Lang.

Bouchard, P. et St-Amant, J.-C. (1996). Garçons et filles : stéréotypes et réussite scolaire. Montréal: Les éditions du Remue-ménage.

Bourdieu, P. (1984). Questions de sociologie. Paris: Minuit.

Bourdieu, P. (1998). La domination masculine. Paris : Seuil.

Bourdieu, P. et Passeron, J.-C. (1970). La reproduction. Eléments pour une théorie du système d'enseignement. Paris: Ed. de Minuit. 
Capdevielle-Mougnibas, V. (2009). La rupture de contrat d'apprentissage: échec ou réussite du parcours de formation? Convention "Expertise Formation " 2009: Centre régional de ressources pour l'apprentissage/ Laboratoire PDPS. Chambre régionale de métiers et de l'artisanat de Midi-Pyrénées. Toulouse (France).

Capdevielle-Mougnibas, V., Cohen-Scali, V., Courtinat-Camps, A., de Léonardis, M., Favreau, C., Fourchard, F. et M. Huet-Gueye, M. (2013). Quelle place pour les apprentis dans les petites entreprise? Représentations de l'apprenti et rapport à la fonction de tuteur des maîtres d'apprentissage dans l'apprentissage de niveau $V$. Toulouse: Université de Toulouse.

Carpentier-Roy, M.-C. (2006). Subjectivité et travail: Pour ne pas dissocier le sujet de l'acteur. In IPDTQ (Ed.), Espace de réflexion, espace d'action en santé mentale au travail. Enquêtes en psychodynamique du travail au Québec (pp. 7-17). Québec: Les Presses de l'Université Laval.

Cart, B., Ducourant, H., Henguelle, V., Surelle, A.-S. et Toutin, M.-H. (2007). Les ruptures des contrats d'apprentissage en Région Nord pas de Calais. Recherche pour le compte du C2RP - Oref.

Cart, B. et Toutin, M.-H. (2015). Rupture de contrat d'apprentissage: interprétations. Diversité, 180 (2), 73-77.

Cassell, J. (2000). Différence par corps : les chirurgiennes. Cahiers du Genre, 29, 53-81.

Castel, R. (1995). Métamorphose de la question sociale: Une chronique du salariat. Paris: Fayard.

Castelli Dransart, A. D., De Puy, J., Perriard, V., Zbinden Sapin, V., Gay-desCombes, B. et Monin, M.-C. (2008). L'identité professionnelle au sein de la formation professionnelle initiale: Représentations collectives de deux professions (polymécanicien et assistant socio-éducatif) chez les apprenants, enseignants, formateurs et informateurs. Rapport final. Fribourg, Lausanne: Université de Fribourg, IFFP, HEF-TS.

Chaix, M.-L. (1996). L'alternance enseignement-travail comme lieu d'observation privilégié des processus de construction identitaire. Education Permanente, 128, 103-115.

Chamberland, L. et Paquin, J. (2008). Sexisme et hétérosexisme:l'expérience de travailleuses lesbiennes exerçant des métiers traditionnellement masculins au Québec. In Y. Guichard-Claudic (Ed.), L'inversion $d u$ genre. Quand les métiers masculins se conjuguent au féminin... et réciproquement (pp. 157-168). Rennes: Presses Universitaires de Rennes.

Chaponnière, C. et Chaponnière, M. (2006). La mixité: des hommes et des femmes. Genève: Infolio éditions. 
COCON. (2006). Einfühlsame, verantwortungsbewusste und anstrengungsbereite Jugend. Retrieved July 22nd, 2016, from http://www.cocon. uzh.ch/wp-content/uploads/newsletter_dez06.pdf.

Cohen-Scali, V. (2000). Alternance et identité professionnelle. Paris: Presses Universitaires de France.

Cohen-Scali, V. (2001). Les attitudes à l'égard de l'insertion professionnelle d'apprentis de l'enseignement supérieur. L'orientation scolaire et professionnelle, 30, 173-202.

Cohen-Scali, V. (2003). The Influence of family, social, and work socialization on the construct of the professional identity of young adults. Journal of Career Development, 29, 237-249.

Collectif. (2003). Mixité: il faut sauver les garçons. Le Monde de l'éducation, $310,28-43$.

Collectif. (2007). Formation professionnelle. Dictionnaire historique suisse. Retrieved from http://www.hls-dhs-dss.ch/textes/f/F13911.php.

Commission du Conseil des Etats. (1884, 31.05). Rapport de la commission du Conseil des Etats à l'Assemblée fédérale concernant l'enquête industrielle, du 3 mai 1884. Feuille fédérale, 4, 934-952.

Conseil Fédéral.(1883). Message du Conseil fédéralàl'Assemblée fédérale sur l'enqête industrielle du 3 décembre 1883. Feuillefédérale, pp. 613-724.

COREL. (1999). Les ruptures de contrat d'apprentissage. Lausanne: Communauté de la Région Lausannoise.

Couppié, T. et Epiphane, D. (2004). Des bancs d'école aux postes de travail... Chronique d'une ségrégation annoncée. Marseille: Céreq.

Couppié, T. et Epiphane, D. (2007). Le chemin des femmes dans les métiers «masculins». In H.Eckert et S. Faure(Eds.), Lesjeunesetl'agencement des sexes (pp. 173-193). Paris: La dispute.

Couppié, T. et Epiphane, D. (2008). Hommes et femmes minoritaires dans leur profession: le bonheur à quel prix? In Y. Guichard-Claudic, D. Kergoat et A. Vilbrod (Eds.), L'inversion du genre. Quand les métiers masculins se conjuguent au féminin... et réciproquement (pp. 41-56). Rennes: Presses Universitaires de Rennes.

Coutant, I. (2007). Délinquance juvénile et rapportaux institutions socio-éducatives. In H. Eckert et S. Faure (Eds.), Les jeunes et l'agencement des sexes (pp. 113-129). Paris: La Dispute.

Croisier, M. (2002). Pionniers, pionnières: Quelle insertion professionnelle? Genève: Office d'orientation et de formation professionnelle.

Dafflon-Novelle, A. (2006). D’avantà maintenant, du bébéàl'adulte :synthèse et implications de la socialisation différenciée des filles et des garçons. In A. Dafflon-Novelle (Ed.), Filles-garçons. Socialisation différenciée? (pp. 361-391). Genève: Presses universitaires de Genève. 
Dallera, C. (2003). Au cœur de la lutte pour le suffrage féminin dans le canton de Vaud. Lucy Dutoit (1868-1937). In C. Dallera et N. Lamamra (Eds.), Du salon à l'usine. Vingt portraits de femmes. Un autre regard surl'histoire du canton de Vaud (pp. 117-132). Lausanne: Ouverture.

Dallera, C. (2007). Eclairages sur la migration féminine économique en Suisse: trois parcours. Nouvelles Questions Féministes, 26(1), 54-69.

Dallera, C. et Ducret, V. (2004). Femmes en formation dans un métier d'homme. Synthèse d'une recherche menée dans le canton de Vaud auprès d'un échantillon d'apprenantes. Lausanne: Bureau de l'égalité entre femmes et hommes du canton de Vaud.

Dallera, C. et Lamamra, N. (2008). Quand les femmes refusent que leurs choix soient socialement déterminés. Vers une nouvelle ségrégation horizontale à l'Université. Cahiers du CERFEE, 25, 183-197.

Daune-Richard, A.-M. et Devreux, A.-M. (1992). Rapports sociaux de sexe et conceptualisation sociologique. Recherches féministes, 5(2), 7-30.

Davezies, P. (1993). Eléments de psychodynamique du travail. Education Permanente, 116(3), 33-46.

Dejours, C. (1996). Centralité du travail et théorie de la sexualité. Adolescence, 14(2), 9-29.

Dejours, C. (1998). Souffrance en France: La banalisation de l'injustice sociale. Paris: Seuil.

Dejours, C. (2000a).Le masculin entre sexualité et société. In D. Welzer-Lang (Ed.), Nouvelles approches des hommes et du masculin (pp. 263-289). Toulouse: Presses Universitaires du Mirail.

Dejours, C. (2000b). Travail, usure mentale: Nouvelle édition augmentée. Paris: Bayard.

Dejours, C. (2001). Le travail entre banalisation du mal et émancipation.1-4. Retrieved July 22nd, 2016 from https://clinicamuncii.files.wordpress. com/2010/06/le-travail-entre-banalization-du-mal-et-emancipation1.pdf.

Delphy, C. (1998). L'Ennemi principal 1, Économie politique du patriarcat. Paris: Syllepse/«Nouvelles Questions féministes».

Delphy, C. (2001).L'Ennemiprincipal2, Penserlegenre. Paris:Syllepse/«Nouvelles Questions féministes».

Depoilly, S. (2014). Filles et garçons au lycée pro. Rapport à l'école et rapport de genre. Rennes: Presses universitaires de Rennes.

Devreux, A.-M. (1984).La parentalité dansle travail: Rôles de sexe et rapports sociaux. In Collectif(Ed.), Le sexe du travail (pp. 113-126). Grenoble: Presses Universitaires.

Devreux, A.-M. (1992). Etre du bon côté. In D. Welzer-Lang et J. P. Filiod (Eds.), Des hommes et du masculin (pp. 147-164). Lyon: Presses universitaires de Lyon. 
Dolch, N. A., Orr, K. E. et Ezernack, J. (2001). Teenage Mothers. In D. L. Peck et N.A. Dolch (Eds.), Extraordinary Behavior: A Case Study Approach to Understanding Social Problems (pp. 95-106). Westport: Praeger.

Dolivo, J.-M. et Rosende H., B. (2000). Ruptures de la solidarité dans le travail salarié. Aspects de la sécurité sociale, bulletin FEAS, 27-35.

Dubar, C. (1996). La socialisation: Construction des identités sociales et professionnelles. Paris: Armand Colin.

Dubar, C. (2000). La crise des identités (2nd ed.). Paris: PUF.

Dubs, R. (2006). Rapportd'expertisesurles questions concernantlaformation professionnelle en Suisse. Berne: h.e.p.

Duc, B. et Lamamra, N. (2014). «Young People'S Occupational Integration Four Years after Dropout from Vocational Education and Training: The Issue of Transition at Stake. A Longitudinal Qualitative Perspective.» In A. Keller, R. Samuel, M. M. Bergmann et N. K. Semmer. (Eds.) Psychological, Educational, and Sociological Perspectives on Success and Well-Being in Career Development, (pp. 45-68) Springer Netherlands.

Ducret, V. (2008). Pour une entreprise sans harcèlement sexuel. Un guide pratique ( $2^{\mathrm{e}} \mathrm{ed}$.). Genève: Georg.

Duru-Bellat, M. (1990). L'école des filles. Quelle formation pour quels rôles sociaux? Paris: L'Harmattan.

Duru-Bellat, M. (1994). Note de synthèse. Filles et garçons àl'école, approches sociologiques et psycho-sociales. $1^{\text {ere }}$ partie: des scolarités sexuées, reflet de différences d'aptitude, ou de différences d'attitudes? Revue Française de Pédagogie, 109, 111-141.

Duru-Bellat, M. (1995a). Au fait... les filles [VHS]. Dijon: Académie de Dijon.

Duru-Bellat, M. (1995b). Note de synthèse. Filles et garçons à l'école, approches sociologiques et psycho-sociales. 2ème partie :la construction scolaire des différences entre les sexes. Revue Française de Pédagogie, 110, 75-109.

Duru-Bellat, M. (1995c). Une facette implicite du métier d'élève. In M. d. Manassein (Ed.), De l'égalité des sexes (pp. 183-201). Paris: Centre national de documentation pédagogique.

Duru-Bellat, M. (2002). Les inégalités sociales à l'école: genèse et mythes. Paris : PUF.

Dymock, D. et Gerber, R. (2002). Unintegrated training? Exploring links between off- and on-the-job learning. Education et Training, 44(1), 23-30.

Eckert, H. (2007). Desfemmes au montage automobile:le difficilearrangement des sexes. In H. Eckert et S. Faure (Eds.), Les jeunes et l'agencement des sexes (pp. 213-229). Paris: La dispute. 
Eckert, H. et Sulzer, E. (2007). Le défi de la féminisation des chaînes automobiles. In H. Eckert et S. Faure (Eds.), Les jeunes et l'agencement des sexes (pp. 195-211). Paris: La dispute.

Eckmann-Saillant, M., Bolzman, C. et De Rham, G. (1994). Jeunessansqualification: Trajectoires, situationsetstratégies. Genève: Les Editions I.E.S.

Ferron, C., Cordonnier, D., Schalbetter, P., Delbos-Piot, I. et Michaud, P.A. (1997). La santé des jeunes en rupture d'apprentissage. Lausanne: Institut universitaire de médecine sociale et préventive.

Fibbi, R., Kaya, B. et Piguet, E. (2003). Nomen est omen: Quand s'appeler Pierre, Afrim ou Mehmet fait la différence. Bern/Aarau: Fonds national Suisse.

Filliettaz, L., De Saint Georges, I. et Duc, B. (2008). «Vos mains sont intelligentes» : Interactions en formation professionnelle initiale (Vol. 117). Genève: Cahiers de la faculté des Sciences de l'Education.

Flahaut, E. et Pennec, S. (2008). Des trajectoires sexuées dans l'accès et le maintien en position atypique. In Y. Guichard-Claudic, D. Kergoat et A. Vilbrod (Eds.), L'inversion du genre. Quand les métiers masculins se conjuguent au féminin... et réciproquement (pp. 31-39). Rennes: Presses Universitaires de Rennes.

Fontagné,L. (2007). La nouvelle division internationale du travail. Universalia 2005. Retrieved from http://ses.ens-lsh.fr/ac07c/0/fiche_article/\&$\mathrm{RH}=\mathrm{SES}$

Forner, Y. et Dosnon, O. (1991). La maturité vocationnelle: Le processus et son évaluation. L'orientation scolaire et professionnelle, 27, 203-218.

Fournier, G. (2002). Interagir : à la découverte de ses représentations du monde du travail et de ses possibilités d'action. Paris: Editions et applications psychologiques.

Fraisse, G. et Perrot, M. (Eds.). (1991). Histoire des femmes en Occident. Le XIXe siècle. Paris: Plon.

Gallioz,S. (2008). Etre femme et entrer dansle secteur du bâtiment: recherche de l'exception ou acte de folie? In Y. Guichard-Claudic, D. Kergoat et A. Vilbrod (Eds.), L'inversion du genre. Quand les métiers masculins se conjuguent au féminin... et réciproquement (pp. 325-339). Rennes: Presses Universitaires de Rennes.

Gasparini, W. (2007). Domination masculine et division sexuelle du travail dans les organisations sportives. In J.-Y. Causer, R. Pfefferkorn et B. Woehl(Eds.), Métiers, identitésprofessionnellesetgenre (pp. 133-148). Paris L'Harmattan.

Gauthier, M. (1994). La construction de l'identité dans un contexte de précarité d'emploi : Le cas des jeunes travailleurs moyennement scolarisés. In J. Hamel et J.-Y. Thériault (Eds.), Les identités: Actes du colloque de l'ACSLAF 1992 (pp. 433-451). Laval: Méridien. 
Gianettoni, L. et Simon-Vermot, P. (2010). Quand la menace d'exclusion professionnelle renforce le genre: représentations et identités de genre auprès de jeunes sans emploi. Nouvelles Questions Féministes, 29(3), 76-90.

Goffman, E. (2002). L'arrangement des sexes. Paris: La Dispute.

Gonon, P., Haefeli, K., Heikkinen, A. et Ludwig, I. (Eds.). (2001). Gender Perspectives on Vocational Education. Historical, Cultural and Policy Aspects. Bern: Peter Lang.

Guichard-Claudic, Y., Kergoat, D. et Vilbrod, A. (2008).Introduction générale. In Y. Guichard-Claudic, D. Kergoat et A. Vilbrod (Eds.), L'inversion du genre. Quand les métiers masculins se conjuguent au féminin... et réciproquement (pp.9-27). Rennes: Presses Universitaires de Rennes.

Guillaumin, C. (1992). Sexe, race et pratique du pouvoir: l'idée de nature. Paris: Côté-Femmes.

Hanhart, S. (2006). Marché de l'apprentissage et pouvoirs publics. Bulletin de la CIIP, 19, 8-9.

Heinz, W. R., Kelle, U., Witzel, A. et Zinn, J. (1998). Vocational training and career development in Germany: Results from a longitudinal study. International Journal of Behavioral Development, 22, 77-101.

Herzog, W., Neuenschwander, M. P. et Wannack, E. (2004). Wie verlaufen Berufswahlprozesse? Panorama, 2, 36-37.

Hidri, O. (2005). (Trans)former son corps, stratégie d'insertion professionnelle au féminin? Formation Emploi, 91, 31-44.

Hirata, H. et Kergoat, D. (1998). La division sexuelle du travail revisitée. In M. Maruani (Ed.), Les nouvelles frontières de l'inégalité (pp. 93-104). Paris: La découverte.

Hirata, H. et Kergoat, D. (2007). Division sexuelle du travail professionnel et domestique. Evolution de la problématique et paradigmes de la «conciliation». In H. Hirata et M. Maruani (Eds.), Actes du colloque international Marchédutravailetgenre: comparaisons internationales Brésil-France. Document de travail du Mage, 10.

Hirigoyen, M.-F. (1998). Le harcèlement moral, la violence perverse au quotidien. Paris: Syros.

hooks, b. (2000). Feminist theory: from margin to center. London: Pluto press. Hughes, E. C. (1996). Le travail et le soi. In J.-M. Chapoulie (Ed.), Le regard sociologique (pp. 75-85). Paris: Editions de l'école des hautes études en sciences sociales.

Hupka-Brunner, S., Grunder, H.-U., Bergman, M. M., Imdorf, C. (eds.) (2015). Qualität in der Bildung. Klinkhardt Verlag. Heilbrunn.

Hurtig, M.-C. (1998). Catégories de sexe et perception de soi. Connexions, 72(2), 105-119. 
Hurtig, M.-C. et Pichevin, M.-F. (2002). Catégorisation de sexe et perception d'autrui. In M.-C. Hurtig, M. Kail et H. Rauch (Eds.), Sexe et genre. De la hiérarchie entre les sexes (pp. 169-180). Paris: CNRS Editions.

Ibos, C. (2008). Les «nounous africaines» et leurs employeurs: une grammaire du mépris social. Nouvelles Questions Féministes, 27(2), 25-38.

Imdorf, C. (2004). La sélection fondée sur le genre dans l'attribution des places de formation. In CSDE (Ed.), Le genre en vue. Les filles et les jeunes femmes face à la formation (pp. 107-120). Berne: Conférence suisse des déléguées à l'égalité.

Imdorf, C. (2007a). Assistants de cabinet de sexe masculin: les médecins et les médecins-dentistes se sentent menacés. Revue Mensuelle Suisse d'Odontostomatologie, 117(3), 332-333.

Imdorf, C. (2007b). La sélection des apprentis dans les PME: Compte rendu mars 2007. Fribourg: Institut de Pédagogie Curative de l'Université de Fribourg.

Imdorf, C., Granato, M., Moreau, G. et G. Waardenburg (2010). Sociology of vocational education and training in Switzerland, France and Germany. Swiss Journal of Sociology, 36(1), 5-10.

Jaboin, Y. (2010). C'est bien... un homme à l'école maternelle! Nouvelles Questions Féministes, 29(2), 34-45.

Jackson, S. (2009). Pourquoi un féminisme matérialiste est (encore) possibleet nécessaire. Nouvelles Questions Féministes, 28(3), 16-33.

Jaunait, A. et S. Chauvin (2012). Représenter l'intersection. Les théories de l'intersectionnalité à l'épreuve des sciences sociales. Revue française de sciences politiques, 1 (62), 5- 20.

Jobin, C. (1995). Entre les activités professionnelle et domestique: la discrimination sexuelle. Genève: Editions d'En bas.

Jost, H. U. (1992). Les avant-gardes réactionnaires. La naissance de la nouvelle droite en Suisse, 1890-1914. Lausanne: Editions d'En Bas.

Joye, D., Bergman, M. M. et Budowski, M. (2007, janvier 2007).Documentation technique du panel suisse de ménages; recodification des variables de position sociale. Retrieved janvier 2007, from http://aresoas.unil.ch/ workingpapers/Wp4_02_F.pdf

Kaiser, C. A., Davaud, C., Evrard, A. et Rastoldo, F. (2007). Les jeunes en formation professionnelle. Rapport II:Commentlesjeunesinterprètent leur parcours de formation. Genève: SRED.

Kergoat, D. (2000). Division sexuelle et rapports sociaux de sexe. In H. Hirata, F. Laborie et H. Le Doaré (Eds.), Dictionnaire critique du féminisme (pp. 35-44). Paris: PUF.

Kergoat, D., Imbert, F., Le Doaré, H. et Senotier, D. (Eds.). (1992). Les infirmières et leur coordination - 1988-1999. Paris: Editions Lamarre. 
Kergoat, P. (2003). Le désenchantement des apprentis facteurs-trieurs. Travailler, 2(10), 107-128.

Kergoat, P. (2006). De l'indocilité au travail d'une fraction des jeunesses populaires. Les apprentis et la culture ouvrière. Sociologie du travail, $48,545-560$.

Kergoat, P. (2011). Les formations par apprentissage dans les grandes entreprises. Saarbrücken: Editions universitaires européennes.

Kergoat, P. (2014). Le travail, l'école et la production des normes de genre. Filles et garçons en apprentissage (en France). Nouvelles Questions féministes, 33 (1), 16-34.

Kergoat, P. (2015). Trouver et tenir sa place. Les apprenti(e)s et le travail. L'orientation scolaire et professionnelle, 44 (2), 207-234.

Kergoat, P. et Capdevielle-Mougnibas, V. (2015). Les formations par apprentissage et la production des inégalités: de l'orientation à la formation. L'orientation scolaire et professionnelle, 44 (2), 135-145.

Lada, E. (2008). Le genre en pratique et pratiques du genre: des hommes dans des emplois de service dits «de femmes». In Y. Guichard-Claudic, D. Kergoat et A. Vilbrod (Eds.), L'inversion du genre. Quand les métiers masculins se conjugent au féminin... et réciproquement (pp. 371-383). Rennes: Presses Universitaires de Rennes.

Lamamra, N. (2011). La formation professionnelle en alternance, un lieu de transmission des normes de genre? Revue Psychologie du travail et des organisations, 17 (4), 330-345.

Lamamra, N. et Duc, B. (2015). Die duale Berufsausbildung. Von einem sanften Übergang zur Arbeitswelt zu einer Anpassung an ihre neuen Mechanismen. In Häfeli, K., Neuenschwander M. et S. Schumann. Berufliche Passagen im Lebenslauf (pp. 101-126). Cham: Springer

Lamamra, N. et Duc, B. (2014). «C'est comme si c'était un parcours de guerre, et puis qu'on ait réussi, puis après on est tranquille...». Lehrvertragsauflösungen als Symptom komplexerer und verlängerter Transitionen. In A. Ryter et D. Schaffner, Wer hilft mir, was zu werden? Professionelles Handeln in der Berufsintegration (pp. 83-99). Bern: HEP.

Lamamra, N., Fassa Recrosio, F. et M. Chaponnière, M. (2014). Formation professionnelle: l'apprentissage des normes de genre. Nouvelles Questions féministes, 33 (1), 8-14

Lamamra, N. et Masdonati, J. (2006). Jeunes en difficulté dans la formation professionnelle: Une question identitaire? Paper presented at the AIPTLF, Hammamet.

Lamamra, N. et Masdonati, J. (2008). Adolescence en souffrance: Stratégies des jeunes face aux contraintes de la formation professionnelle. Reflets - Revue d'intervention sociale et communautaire, 14, 67-102. 
Lamamra, N. et Masdonati, J. (2009a). Arrêter une formation professionnelle: Mots et maux d'apprenti.e.s. Lausanne: Antipodes.

Lamamra, N. et Masdonati, J. (2011a). Les arrêts prématurés en formation professionnelle. Entre échec de la transition et parcours non-linéaires vers l'insertion professionnelle. In A. Pilote et J. Masdonati (Eds.), Parcours scolaires et parcours d'insertion socio-professionnelle des jeunes (titre provisoire). Québec: Presses de l'Université Laval.

Lamamra, N. et Masdonati, J., (2011b). Les apprenti.e.s face aux contraintes en formation professionnelle en alternance: entre souff rance et plaisir. Revue Psychologie du travail et des organisations, 17 (3), 291-206.

Lamamra, N. et Moreau, G. (2016). Le Certificat fédéral de capacité: la fin d'une centralité? Formation Emploi, L'apprentissage en Suisse, 133, 101-120.

Lamamra, N. et Rosende, M. (2005). Quand l'égalité se heurte aux rôles sociaux de sexe. L'exemple de la campagne Tekna. Nouvelles Questions Féministes, 24(1), 114-117.

Laufer, J. (2009). L'égalité professionnelle entre les hommes et les femmes est-elle soluble dans la diversité? Travail, genre et sociétés, 21, 29-54.

Lave, J. et Wenger, E. (2002). Situated learning: Legitimate peripheral participation (10th ed.). Cambridge: University Press.

Le Feuvre, N. (2008). La féminisation des anciens «bastions masculins»: enjeux sociaux et approches sociologiques. In Y. Guichard-Claudic, D. Kergoat et A. Vilbrod (Eds.), Quand les métiers masculins se conjugent au féminin... et réciproquement (pp. 307-323). Rennes: Presses Universitaires de Rennes.

Le Feuvre, N. et Laufer, J. (2008). Quand l'avancée en mixité est le fait des hommes. In Y. Guichard-Claudic, D. Kergoat et A. Vilbrod (Eds.), L'inversion du genre. Quand les métiers masculins se conjuguent au féminin... et réciproquement (pp. 207-213). Rennes: Presses Universitaires de Rennes.

Leclerc, C. et Maranda, M.-F. (2002). The psychodynamics of work: Action research in an academic setting. Canadian Journal of Counselling Revue canadienne de counseling, 36, 194-210.

Leemann, R. J. et Keck, A. (2004). Attentes et attitudes des filles face au choix professionnel et à un projet de vie. In Conférence suisse des déléguées à l'égalité (Ed.), Le genre en vue. Les filles et les jeunes femmes face à la formation (pp. 31-44). Berne: Conférence suisse des déléguées à l'égalité.

Legault, M.-J. (2001). Violence auprès des femmes dans les secteurs d'emploi non traditionnellement féminins et indemnisation. Pistes, 3(1). Retrieved from http://www.pistes.uqam.ca/v3n1/articles/v3nla3.htm 
Lemarchant, C. (2007). Garçons et filles atypiques au sein des formations professionnelles de niveaux IV et V. In J.-Y. Causer, R. Pfefferkorn et B. Woehl(Eds.), Métiers, identités professionnellesetgenre (pp. 51-64). Paris: L'Harmattan.

Lemarchant, C. (2008). Unique en son genre... Orientations atypiques de lycéens et lycéennes au sein des filières techniques et professionnelles. In Y. Guichard-Claudic, D. Kergoat et A. Vilbrod (Eds.), Quand les métiers masculins se conjuguent au féminin... et réciproquement (pp. 57-69). Rennes: Presses Universitaires de Rennes.

Levy, R., Joye, D., Guye, O. et Kaufmann, V. (1998). Inégalités sociales en Suisse: Un aperçu. Zürich: Seismo.

Lhuilier, D. (2005). Le «sale boulot». Travailler, 14, 73-98.

LINK. (2005). Baromètre de l'apprentissage: Rapport condensé. Retrieved July 19, 2005, from www.bbt.admin.ch

Malochet, G. (2008). Surveillantes surveillées: la mixité au travail dans les prisons pour hommes. In Y. Guichard-Claudic, D. Kergoat et A. Vilbrod (Eds.), L'inversion du genre. Quand les métiers masculins se conjuguent au féminin... et réciproquement (pp. 133-144). Rennes: Presses Universitaires de Rennes.

Maranda, M.-F. (2003). Etre un acteur ou un sujet? Voilà la question. Enquête sur la psychodynamique du travail de conseiller en emploi. In G. Fournier, B. Bourassa et K. Béji (Eds.), La précarité du travail. Une réalité aux multiples visages (pp. 163-187). Québec: Les Presses de l'Université Laval.

Maranda, M.-F. et Leclerc, C. (2000). Les jeunes et le discours morose sur l'emploi: Perspective de la psychodynamique du travail. In G. Fournier et M. Monette (Eds.), L'insertion socioprofessionnelle: Un jeu de stratégie ou un jeu de hasard? (pp. 107-124). Sainte-Foy (Canada): Les Presses de l'Université Laval.

Maranda, M.-F. et Viviers, S. (2011). L'école en souffrance. Psychodynamique du travail en milieu scolaire. Québec Les Presses de l'Université Laval.

Marry, C. (2004a). La réussite des filles à l'école. Trois interprétations sociologiques. Sciences humaines, 146, 34-35.

Marry, C. (2004b). Les femmes ingénieurs, une révolution respectueuse. Paris: Belin.

Marry, C. (2006). Variations sociologiques sur le sexe des métiers. In C. Vidal(Ed.), Mythesscientifiqueset idéologies (pp. 83-93). Paris: Belin.

Marry, C. (2007). Celles qui dérogent.... In C. Baudelot et R. Establet (Eds.), Quoi de neuf chez les filles? Entre stéréotypes et liberté (pp. 123-128). Paris: Nathan. 
Marry, C. (2008). Les définitions du genre et leurs enjeux en situation d'inversion. In Y. Guichard-Claudic, D. Kergoat et A. Vilbrod (Eds.), L'inversion du genre. Quand les métiers masculins se conjuguent au féminin... et réciproquement (pp. 299-306). Rennes: Presses Universitaires de Rennes.

Martin, H. et Roux, P. (2015). Recherches féministes sur l'imbrication des rapports de pouvoir : une contribution à la décolonisation des savoirs. Nouvelles Questions féministes 34 (1), 4-13.

Maruani, M. (2006). Travailetemploidesfemmes ( $3^{\mathrm{e}}$ ed.). Paris:La Découverte. Maruani, M. et Nicole, C. (1987). Du travail à l'emploi : l'enjeu de la mixité. Sociologie du travail, 2, 237-250.

Maruani, M. et Nicole, C. (1989). Clavistes et correcteurs: nouvelles technologies et vieux métiers. In M. Maruani et C. Nicole (Eds.), Au labeur des dames (pp. 17-57). Paris: Syros.

Masdonati, J. et Lamamra, N. (2009). La relation entre apprenti.e et personne formatrice au coeur de la transmission des savoirs en formation professionnelle. Revue suisse des sciences de l'éducation, 31(2), 335-353.

Masdonati, J., Lamamra, N. et Jordan, M. (2010). Vocational Education and Training Attrition and the School-to-Work Transition. Education + Training, 52(5), 404-414.

Massoudi, K. (2009). Le stress professionnel: une analyse des vulnérabilités individuelles et des facteurs de risque environnementaux. Bern: Peter Lang.

Mauger, G. (2006). Les bandes, le milieu et la bohème populaire. Etude de la sociologie de la déviance des jeunes des classes populaires (1975-2005). Paris: Belin.

Mauger, G. et Poliak, C. (1983). Les loubards. Actes de la recherche en sciences sociales, 50, 49-68.

Mayeur, F. (1991). L'éducation des filles : le modèle läque. In G. Fraisse et M. Perrot (Eds.), Histoire des femmes. Le XIXe siècle, Vol. 4, pp. 231-248. Paris: Plon.

Meeus, W., Dekovic, M. et Iedema, J. (1997). Unemployment and identity in adolescence: A social comparison perspective. The Career Development Quarterly, 45, 369-380.

Mègemont, J.-L. et Baubion-Broye, A. (2001). Dynamiques identitaires et représentations de soi dans une phase de transition professionnelle et personnelle. Connexions, 76(2), 15-28.

Messant-Laurent, F. (1990). L'épouse de bureau. In F. Messant-Laurent (Ed.), La secrétaire modèle (pp. 97-113). Paris: L'Harmattan. 
Meyer, T. (2003). When being smart is not enough: Institutional and social access barriers to uppersecondaryeducation and theirconsequences on successfullabourmarketentry. The case of Switzerland. Paper presented at the 2003 Workshop "Competencies and Careers" of the European Research Network on Transitions in Youth, Funchal, Madeira.

Meyer, T. (2004). Wie weiter nach der Schule? - L'école... et après? Leitungsgruppe des NFP 43 in Zusammenarbeit mit dem Forum Bildung und Beschäftigung und der Schweizerischen Koordinationsstelle für Bildungsforschung (SKBF). Retrieved July 22, 2016, from http://www. snf.ch/sitecollectiondocuments/nfp/nfp43_meyer_synthesis6.pdf.

Meyer, T. (2005). Passage à l'emploi: jeunes en transition de la formation au marché du travail. Résultats globaux de la recherche longitudinale TREE, état 2004. Berne: TREE.

Michaud, P.-A. (2001). Prévenir les ruptures, limiter leurs conséquences. Panorama, 6, 8-10.

Modak, M., de Kinkelin, C. et Benelli, N. (2008). La reconnaissance du care dans le travail social: éléments pour une analyse du travail des assistant.e.s sociaux. In M. Rosende et N. Benelli (Eds.), Laboratoires $d u$ travail (pp. 105-116). Lausanne: Antipodes.

Molinier, P. (2000). Virilité défensive, masculinité créatrice. Travail, Genre et Sociétés. Le genre masculin n'est pas neutre, 3, 25-44.

Molinier, P. (2002a). Féminité sociale et construction de l'identité sexuelle: Perspectives théoriques et cliniques en psychodynamique du travail. L'Orientation Scolaire et Professionnelle, 31, 565-580.

Molinier, P. (2002b). Le continent noir de la féminité: sexualité ou travail? Cliniques méditerranéennes, 66, 105-123.

Molinier, P. (2004). Psychodynamique du travail et rapports sociaux de sexe. Travail et Emploi, 97, 79-91.

Molinier, P. et Dejours, C. (1997). Psychodynamique du travail et psychologie clinique du chômage. Psychologie Française, 42, 261-268.

Molinier, P. et Grenier-Pezé, M. (2000). Introduction. Cahiers du Genre, 29, 7-19.

Moreau, G. (2000). Les faux semblables de l'apprentissage. Travail Genres et Sociétés. Le genre masculin n'est pas neutre, 3, 67-86.

Moreau, G. (2003). Le monde apprenti. Paris: La Dispute.

Moreau, T. (2002). Ecrire les genres. Guide romand d'aide à la rédaction administrative et législative épicène. Lausanne: Conférence latine des déléguées à l'égalité.

Mosconi, N. (1994). Femmes et savoir. La société, l'école et la division sexuelle des savoirs. Paris: L’Harmattan. 
Moser, C., Stalder, B. E. et Schmid, E. (2008). Lehrvertragsauflösung: Die Situation von ausländischen und Schweizer Lernenden. Berne: Erziehungsdirektion des Kantons Bern.

Moser, U. (2004). Jugendliche zwischen Schule und Berufsbildung: Eine Evaluation bei Schweizer Grossunternehmen unter Berücksichtigung des internationalen Schulleistungsvergleichs PISA. Leitungsgruppe des NFP 43. Retrieved July 22, 2016, from http://www.snf.ch/SiteCollectionDocuments/nfp/nfp43_moser_synthesis20.pdf.

Moulin, C. (2005). Féminités adolescentes: Itinéraires personnels et fabrication des identités sexuées. Rennes: Presses universitaires de Rennes.

Neuenschwander, M. P. (1999). Lehrvertragsauflösungen im Kanton Zürich: Schlussbericht. Zürich: Verlag Impulse; Mittelschul- und Berufsbildungsamt/Bildungsentwicklung.

Neuenschwander, M. P. et Stalder, B. E. (1998). Lehrvertragsauflösungen aus derSicht von Jugendlichen: Ergebnisse einer DeutschschweizerStudie. Berufsbildung international, BWP 27, 42-47.

Neuenschwander, M. P., Stalder, B. E. et Süss, D. (1996). Berufswahl und Lehrvertragsauflösungen im Kanton Bern. Bern: Amt für Bildungsforschung.

Nicole-Drancourt, C. et Roulleau-Berger, L. (2002). L'insertion des jeunes en France. Paris: PUF.

OCDE.(2008).Regardssurl'éducation 2008:Panorama. Retrievedfromhttp:// www.oecd-ilibrary.org/sites/9789264040649-fr/01/08/index.html.

OFS. (2015). Egalité entre femmes et hommes - Données, indicateurs. Choix professionnels et desétudes. Neuchâtel: Office fédéral dela statistique, from http://www.bfs.admin.ch/bfs/portal/fr/index/themen/20/05/ blank/key/gleichstellung_und/eintritte_in_berufsbildungen.html.

OFS. (2013). Enseignants selon le canton, le degré d'enseignement et le sexe (écolespubliques), en 2010/11. Neuchâtel:Office fédéraldela statistique.

OFS. (2004). Vers l'égalité? Neuchâtel: Office fédéral de la statistique.

OFS. (2008a). Participation à la formation: Âge moyen des nouveaux apprenants. Retrieved 18 décembre 2008, from http://www.bfs.admin.ch/ bfs/portal/fr/index/themen/15/04/ ind4.indicator.40703.407.html.

OFS. (2008b). Versl'égalité2008. Womenandmen in OECD countries. Retrieved 03.08.2010, from http://www.oecd.org/dataoecd/44/52/37962502.pdf.

OMS. (2002). Directives générales sur la violence au travail dans le secteur de la santé. Genève: Organisation mondiale de la santé.

Palheta, U. (2015). Le salut hors de l'école? Comprendre l'orientation vers l'apprentissage. Diversité, 180 (2), 66-72.

Palmonari, A. et Sarchielli, G. (1993). Evoluzione degli studi sull'adolescenza. In A. Palmonari (Ed.), Psicologia dell'adolescenza (pp. 15-42). Bologna: Il Mulino. 
Parini, L. (2006). Le système de Genre: introduction aux concepts et théories. Zurich: Seismo.

Paugam, S. (2003). Les nouvelles précarités du travail. In G. Fournier, B. Bourrassa et $\mathrm{K}$. Béji (Eds.), La précarité du travail. Une réalité aux multiples visages (pp.3-21).Québec:Les Presses del'Université Laval.

Pfefferkorn, R. (2007). Le travail au coeur des rapports sociaux de sexe. In J.-Y. Causer, R. Pfefferkorn et B. Woehl (Eds.), Métiers, identités professionnelles et genre (pp. 11-29). Paris: L'Harmattan.

Pfefferkorn, R. (2008). Quand l'avancée en mixité est le fait des femmes. In Y. Guichard-Claudic, D. Kergoat et A. Vilbrod (Eds.), L'inversion du genre. Quand les métiers masculins se conjuguent au féminin... et réciproquement (pp. 113-119). Rennes: Presses Universitaires de Rennes.

Pfister Giauque, B. et Flamigni, E. (2010). Hétérogénéité culturelle et sociale, non-mixitéet intégration de la différence dans la formation professionnelle. Rapport final. Lausanne/Fribourg: IFFP/Leadinghouse QVET.

Pheterson, G. (2001). Le prisme de la prostitution. Paris: L'Harmattan.

Poglia, E. (1999). Globalisation : terrain miné ou terrain fertile pour l'éducation? In M. Carton, S. Hanhart, S. Perez, E. Poglia et J. Terrier (Eds.), Globalisation économique et systèmes de formation en Suisse. Actes du colloque du 8 décembre 1998). Cahiers de la section des sciences de l'éducation, 90, pp. 25-45.

Probst, I. (2004). La souffrance au travail. Une comparaison de différentes approches théoriques dans une perspective psychosociale. Lausanne: Université de Lausanne.

Quiroga, C., Janosz, M. et Marcotte, D. (2006). Les sentiments dépressifs à l'adolescence: Un facteur de risque différentiel du décrochage scolaire chez les filles et les garçons de milieu défavorisé. Revue de psychoéducation, 35, 277-300.

Rastoldo, F. (2006). Les élèves de la 9e s'expriment sur leurs choix d'orientation. Panorama, 1, 19-20.

Rastoldo, F., Amos, J. et Davaud, C. (2009). Les jeunes en formation professionnelle; Rapport III: Le devenir des jeunes abandonnant leur apprentissage. Genève: SRED.

Rastoldo, F., Evrard, A. et Amos, J. (2007). Les jeunes en formation professionnelle. Rapport I: Entrée en formation professionnelle et parcours de formation des diplômés. Genève: SRED.

Rastoldo, F., Kaiser, C. A. et Evrard, A. (2008). Les jeunes en formation professionnelle. RapportII:Commentlesjeunes interprètentleurparcours de formation. Genève: SRED.

Rédaction. (2008). Les femmes exerçant des professions masculines interrompent plus souvent leur apprentissage. Panorama, 5, 21. 
Renard, F. (2015). Entre revanche scolaire et subordination salariale. Les appropriations ambivalentes du statut d'apprenti chez des coiffeurs en CAP. L'orientation scolaire et professionnelle, 44 (2), 235-267.

Renevey-Fry, C. (Ed.). (1997). En attendant le prince charmant. L'éducation des jeunes filles à Genève1740-1970. Genève: Service de la recherche en éducation (SRED).

Rochat, S. et Lamamra, N. (2004). La socialisation des apprenti.e.s. Panorama, 4, 21-22.

Rogel, T. (2004). Introduction impertinente à la sociologie (2ème ed.). Paris: Liris.

Rosende, M. (2008). Parcours féminins et masculins de spécialisation en médecine. Zurich: Seismo.

Rosenthal, B. S. (1998). Non-school correlates of dropout: An integrative review of theliterature. Childrenet Youth Services Review, 20,413-433.

Roux, P. (2000). Des femmes au genre: que penser de la différence des sexes? Dépendances, 11, 1-4.

Roux,P.(2006).Etudes Genre:Uneperspectivedéstabilisatricedel'ordre social sexué. In M. Modak et J.-P. Tabin (Eds.), Penser le social. Hommages à Jean-Pierre Fragnière (pp. 25-38). Lausanne: Les Cahiers de l'éésp.

Roux, P., Gianettoni, L. et Perrin, C. (2006). Féminisme et racisme. Une recherche exploratoire sur les fondements des divergences relatives au port du foulard. Nouvelles Questions Féministes, 25(1), 84-106.

Salman, S. (2008). Fortune d'une catégorie: la souffrance au travail chez les médecins du travail. Sociologie du travail, 50, 31-47.

Schmid, E. (2010). Kritisches Lebensereignis "Lehrvertragsauflösung». Eine Längsschnittuntersuchung zum Wiedereinstieg und zum subjektiven Wohlbefinden betroffener Jugendlicher. Bern: h.e.p.

Schmid, E. et Stalder, B. E. (2007). Lehrvertragsauflösung: Direkter Wechsel und vorläufiger Ausstieg: Ergebnisse aus dem Projekt LEVA. Berne: Bildungsplanung und Evaluation der Erziehungsdirektion des Kantons Bern.

Scott, J. (1988). Genre: une catégorie utile d'analyse historique. Cahiers $d u$ GRIF, 37-38.

Scott, J. (1991). La travailleuse. In G. Fraisse et M. Perrot (Eds.), Histoire des femmes. Le XIXe siècle, 4, 419-444. Paris: Plon.

SECO. (2005). Mobbing: Description et aspects légaux. Berne: Secrétariat d'Etat à l'économie (SECO).

SEFRI. (2015). La formation professionnelle en Suisse. Faits et chiffres 2015. Berne: Secrétariat d'Etat à la formation, à la recherche et à l'innovation - SEFRI. 
Solar, C. (1993). Femmes et savoir. Paper presented at the Institut Romand de Recherches et de Documentation Pédagogique et le Collège de Psychologie et des Sciences de l'Education, Université de Neuchâtel.

Stalder, B. E. et Schmid, E. (2006a). Lehrvertragsauflösungen, ihre Ursachen und Konsequenzen:Ergebnisse aus dem Projekt LEVA. Berne:Bildungplanung und Evaluation der Erziehungsdirektion des Kantons Bern.

Stalder, B. E. et Schmid, E. (2006b). Raisons et conséquences des abandons d'apprentissage. Panorama, 2(2006), 13-14.

Sudbury, Julia (1998). "OtherKinds ofDreams": Black Women's Organizations and the Politics of Transformation. London: Routledge.

Tabet, P. (1979). Les Mains, les outils, les armes. L'Homme, 19(3-4), 5-61.

Terrail, J.-P. (1990). Destins ouvriers. La fin d'une classe? Paris: PUF.

Testenoire, A. (2008). Carrières féminines, résistances masculines: couples à hypogamie féminine. In Y. Guichard-Claudic, D. Kergoat et A. Vilbrod (Eds.), L'inversion du genre. Quand les métiers masculins se conjuguent au féminin... et réciproquement (pp. 385-396). Rennes: Presses Universitaires de Rennes.

Thiébaud, J. (2004). L'intégration professionnelle de pionniers et pionnières: une approche comparative. Université de Lausanne.

Trottier, C. (2006, Juillet). Insertion professionnelle et rapport au temps de jeunes ayant interrompu leurs études secondaires. Paper presented at the AIPTLF, Hammamet.

Vézina, M. (2007). Les fondements théoriques de la psychodynamique du travail. In M. Neboit et M. Vézina (Eds.), Stress au travail et santé psychique (pp. 111-118). Toulouse: Octarès.

Vonthron, A.-M., Lagabrielle, C. et Pouchard, D. (2007). Le maintien en formation professionnelle qualifiante: Effets de déterminants motivationnels, cognitifs et sociaux. L'orientation scolaire et professionnelle, $36,401-420$.

Vouillot, F. (1999). Orientation: le reflet des rôles de sexe. In F. Vouillot (Ed.), Filles et garçons à l'école: une égalité à construire (pp. 81-90). Paris: CNDP.

Vouillot, F. (2002). Réflexions sur la division sexuée de l'orientation. Panorama, 4, 27-29.

Vouillot, F. (2007). L'orientation aux prises avec le genre. Travail, genre et société. Formation et orientation: l'empreinte du genre, 18(2), 87-108.

Waardenburg, G. (2007). Passer del'école au travail productif.La reproduction sociale par la voie suisse de l'apprentissage professionnel. In M. Roca i Escoda et N. Panayotopoulos (Eds.), Structures sociales en transformation. Approches théoriques, méthodologiques et empiriques. Actes de la IIe Ecole d'été ESSE (pp. 102-111): Université de Crète. 
Welzer-Lang, D. (2000). Nouvelles approches des hommes et du masculin. Toulouse: Presses du Mirail.

West, C. et Zimmerman, D. H. (1987). Doing Gender. Gender and Society, $1(2), 125-151$.

West, C. et Zimmerman, D. H. (2009). Faire le genre. Nouvelles Questions Féministes, 28(3), 34-61.

Wittig, M. (2001). La pensée straight (éd. originale 1992). Paris: Balland.

Zaidman, C. (1996). Liberté, égalité, mixité! In C. Zaidman (Ed.), La mixité à l'école primaire (pp. 205-213). Paris: L'Harmattan.

Zaidman, C. (2007). Genre et socialisation. Un parcours intellectuel. Paris: Université Paris Diderot.

Zittoun, T. (2006). Insertions: A quinze ans, entre échec et apprentissage. Collection Exploration. Bern: Peter Lang. 


\section{Annexes}

\section{Annexe 1 \\ Les métiers pionniers* dans le canton de Vaud, en 2014}

\begin{tabular}{|c|c|c|c|}
\hline \multicolumn{2}{|l|}{ Filles $^{* *}$} & \multicolumn{2}{|l|}{ Garçons $^{* * *}$} \\
\hline Maraîcher CFC & $30.0 \%$ & Gardien d'animaux CFC & $30.0 \%$ \\
\hline Maréchal-ferrant CFC & $25.0 \%$ & Polygraphe CFC & $28.6 \%$ \\
\hline Techniscéniste CFC & $25.0 \%$ & Photographe & $28.6 \%$ \\
\hline Géomaticien CFC & $23.3 \%$ & Fleuriste AFP & $25.0 \%$ \\
\hline Dessinateur-constructeur industriel CFC & $23.1 \%$ & Spécialiste en photographie CFC & $25.0 \%$ \\
\hline Horticulteur CFC & $20.3 \%$ & Droguiste CFC & $25.0 \%$ \\
\hline Technologue du lait CFC & $20.0 \%$ & Assistant socio-éducatif CFC & $24.9 \%$ \\
\hline Projeteur en technique du bâtiment sanitaire CFC & $20.0 \%$ & Photographe CFC & $20.0 \%$ \\
\hline Horloger-praticien & $19.0 \%$ & Créateur de vêtements & $19.0 \%$ \\
\hline Viticulteur CFC & $18.8 \%$ & Assistant en soins et santé communautaire CFC & $16.8 \%$ \\
\hline Mécanicien de production CFC & $18.8 \%$ & Agent relation client CFC & $16.7 \%$ \\
\hline Opérateur de machines automatisées CFC & $16.7 \%$ & Aide en soins et accompagnement AFP & $16.7 \%$ \\
\hline Laborant en physique & $16.7 \%$ & Coiffeur AFP & $15.4 \%$ \\
\hline Carrossier-peintre CFC & $15.9 \%$ & Assistant de bureau AFP & $15.0 \%$ \\
\hline Carreleur CFC & $14.3 \%$ & Gestionnaire en information documentaire CFC & $14.3 \%$ \\
\hline Ass.vernisseuse & $14.3 \%$ & Coiffeur CFC & $12.8 \%$ \\
\hline Dessinateur-constructeur sur métal CFC & $14.3 \%$ & Spécialiste en hôtellerie CFC & $12.5 \%$ \\
\hline Agriculteur CFC & $12.5 \%$ & Polydesigner 3D CFC & $11.1 \%$ \\
\hline Menuisier-ébéniste & $12.5 \%$ & Gestionnaire en intendance CFC & $7.5 \%$ \\
\hline Télématicien CFC & $12.5 \%$ & Assistant en pharmacie CFC & $7.0 \%$ \\
\hline Agent de propreté CFC & $12.5 \%$ & Assistante en médecine vétérinaire CFC & $6.7 \%$ \\
\hline Vitrier CFC & $10.0 \%$ & & \\
\hline $\begin{array}{l}\text { Projeteur en technique du bâtiment chauffage } \\
\text { CFC }\end{array}$ & $10.0 \%$ & & \\
\hline Agent de propreté AFP & $10.0 \%$ & & \\
\hline Formation élémentaire: Bâtiment, peinture & $10.0 \%$ & & \\
\hline Monteur automaticien CFC & $9.1 \%$ & & \\
\hline Peintre en bâtiment & $9.0 \%$ & & \\
\hline Ebéniste/Menuisier CFC & $8.5 \%$ & & \\
\hline Constructeur d'appareils industriels CFC & $8.3 \%$ & & \\
\hline Logisticien AFP & $7.1 \%$ & & \\
\hline Logisticien CFC & $7.1 \%$ & & \\
\hline Mécatronicien d'automobiles CFC & $6.9 \%$ & & \\
\hline Boucher-charcutier CFC & $6.3 \%$ & & \\
\hline Informaticien CFC & $6.3 \%$ & & \\
\hline
\end{tabular}

Continuation du tableau sur la prochaine page. 
Continuation du tableau Annex 1.

\begin{tabular}{ll|l}
\hline Filles $^{* *}$ & & Garçons $^{* * *}$ \\
\hline Automaticien CFC & $5.9 \%$ & \\
Horticulteur AFP & $5.6 \%$ & \\
Mécanicien en machines agricoles CFC & $4.8 \%$ & \\
Mécanicien en maintenance d'automobiles CFC & $4.2 \%$ & \\
Assistant en maintenance d'automobiles AFP & $4.2 \%$ & \\
Electronicien CFC & $4.2 \%$ & \\
Agent d'exploitation CFC & $4.2 \%$ & \\
Installateur en chauffage CFC & $3.3 \%$ & \\
Forestier-bûcheron CFC & $2.2 \%$ & \\
Installateur sanitaire CFC & $2.0 \%$ & \\
Electricien de montage CFC & $1.1 \%$ & \\
\hline
\end{tabular}

Source: SCRIS, 2015 (http ://www.scris.vd.ch)

* Ce tableau se base sur le taux pris en considération par le Secrétariat à la condition féminine du Gouvernement du Québec, c'est-à-dire qu'un métier est considéré comme pionnier lorsque la proportion de l'un ou l'autre sexe est inférieure à 33\%. Ces données sont tirées du service cantonal de recherche et d'information statistiques du Canton de Vaud.

** Pour la volée 2014, les métiers suivant ne comportent aucune fille (taux de féminisation=0\%): Agropraticien AFP, Aviculteur CFC, Arboriculteur CFC, Assistant en boucherie et charcuterie AFP, Agent d'entretien de bateaux, Praticien sur bois AFP, Aide-menuisier AFP, Charpentier, Charpentier CFC, Cordonnier CFC, Marbrier CFC, Tailleur de pierre CFC, Mécanicien en machines de chantier CFC, Carrossier-tôlier CFC, Installateur-électricien CFC, Mécanicien en cycles CFC, Serrurier sur véhicules CFC, Aide en technique du bâtiment AFP, Aide en informatique AFP, Monteur frigoriste CFC, Projeteur frigoriste CFC, Constructeur d'installations de ventilation CFC, Praticien en mécanique AFP, Aide-constructeur métallique AFP, Constructeur métallique CFC, Mécanicien d'appareils à moteur CFC, Mécanicien en motocycles CFC, Electronicien en multimédia CFC. Electricien de réseau CFC, Polymécanicien CFC, Praticien en pneumatique AFP, Ferblantier CFC, Technologue en assainissement CFC, Micromécanicien, Micromécanicien CFC, Aide-maçon AFP, Poseur de revêtements de sols CFC, Aide-polybâtisseur AFP, Polybâtisseur CFC, Plâtrier, Plâtrier-peintre, Maçon CFC, Poêlier-fumiste CFC, Aide-carreleur AFP, Constructeur de routes CFC, Constructeur de voies ferrées CFC, Paveur CFC. Constructeur de fondations CFC, Constructeur de sols industriels et chapes CFC, Assistant-constructeur de routes AFP, Vernisseur industriel CFC, Maquettiste d'architecture, Planificateur-électricien CFC, Projeteur en technique du bâtiment ventilation CFC, Laboriste, Dessinateur-constructeur en microtechnique, Conducteur véhicules lourds CFC, Ramoneur CFC, Recycleur CFC, Formation élémentaire: Autres professions.

*** Pour la volée 2014, les métiers suivant ne comportent aucun garçon (taux de masculinisation=0\%): Fleuriste CFC, Professionnel du cheval CFC, Décorateur d'intérieurs, Orthopédiste CFC, Employé en intendance AFP, Employé en hôtellerie AFP, Esthéticienne CFC, Assistant dentaire CFC, Assistante médicale CFC 
Annexe 2

Les 12 professions les plus choisies par les jeunes, en 2014

\begin{tabular}{lr|lr}
\hline Filles & & Garçons & \\
\hline Employé de commerce CFC & $28.8 \%$ & Employé de commerce CFC & $14.1 \%$ \\
Assistant socio-éducatif CFC & $9.9 \%$ & Informaticien CFC & $4.7 \%$ \\
Gestionnaire du commerce de détail CFC & $7.4 \%$ & Gestionnaire du commerce de détail CFC & $4.2 \%$ \\
Assistant en soins et santé communautaire CFC & $6.7 \%$ & Mécanicien en maintenance d'automobiles CFC & $3.6 \%$ \\
Assistant en pharmacie CFC & $4.0 \%$ & Installateur-électricien CFC & $3.4 \%$ \\
Assistante médicale CFC & $2.7 \%$ & Logisticien CFC & $2.7 \%$ \\
Assistant dentaire CFC & $2.6 \%$ & Cuisinier CFC & $2.7 \%$ \\
Coiffeur CFC & $2.6 \%$ & Automaticien CFC & $2.5 \%$ \\
Cuisinier CFC & $2.1 \%$ & Horticulteur CFC & $2.4 \%$ \\
Assistant du commerce de détail AFP & $1.9 \%$ & Electronicien CFC & $2.4 \%$ \\
Gestionnaire en intendance CFC & $1.8 \%$ & Polymécanicien CFC & $2.3 \%$ \\
Gestionnaire du commerce de détail CFC - & & & \\
gestion & $1.8 \%$ & Assistant socio-éducatif CFC & $2.3 \%$ \\
& & & $3 \times 840$ \\
\hline
\end{tabular}

Source: SCRIS, 2015 (http ://www.scris.vd.ch) 

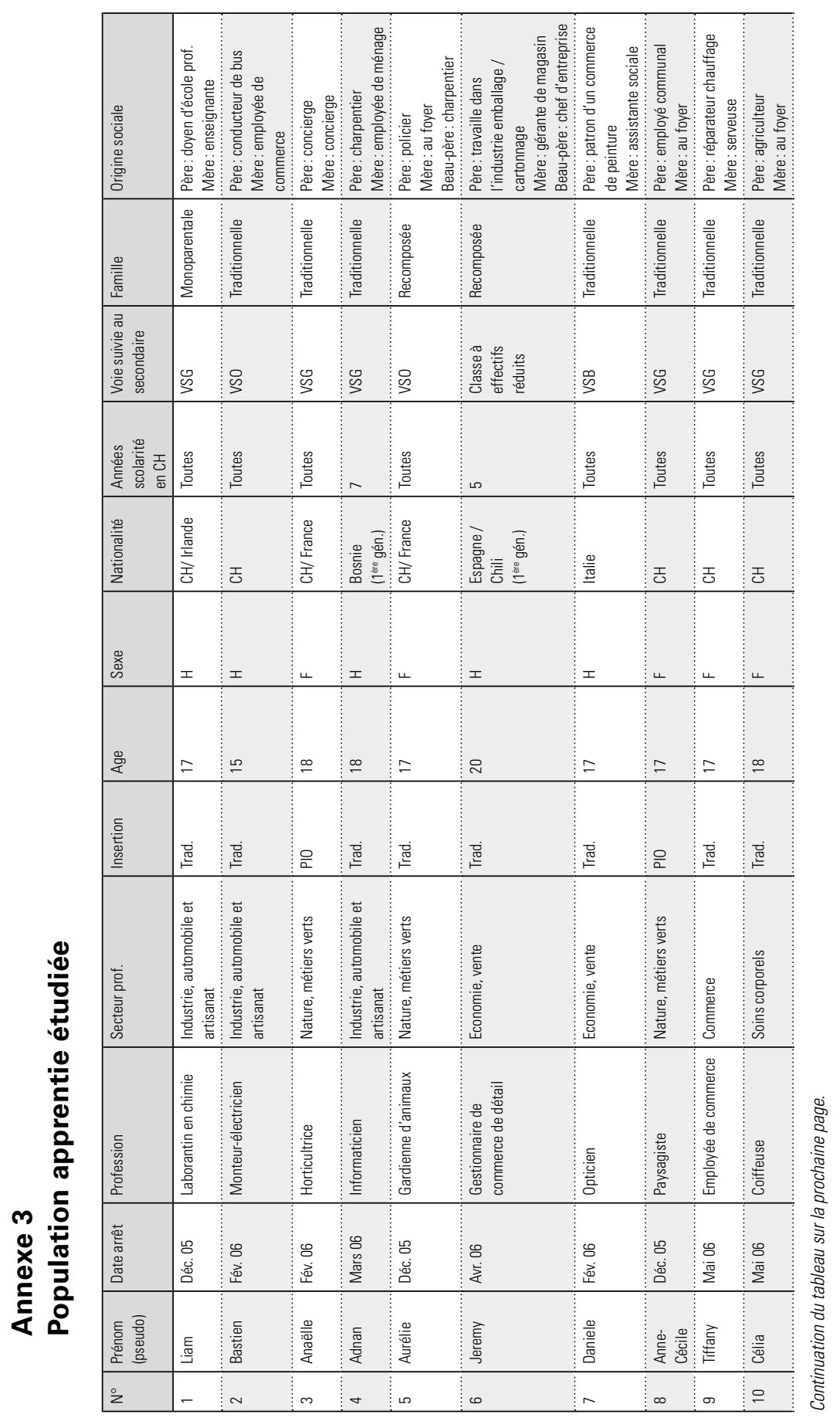


\begin{tabular}{|c|c|c|c|c|c|c|c|c|c|c|c|c|}
\hline 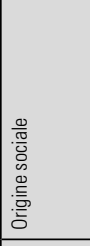 & 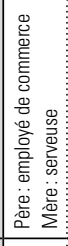 & 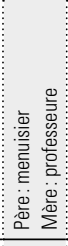 & 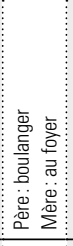 & 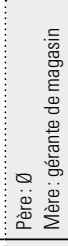 & 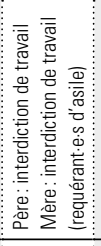 & 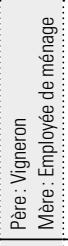 & 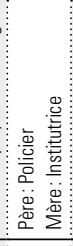 & 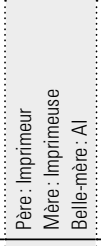 & 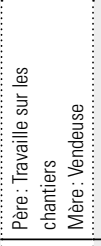 & 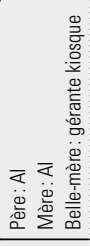 & 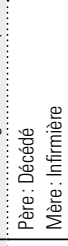 & 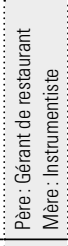 \\
\hline 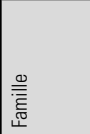 & 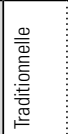 & 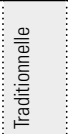 & 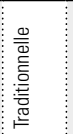 & 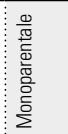 & 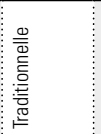 & 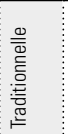 & 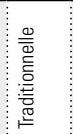 & 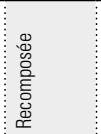 & 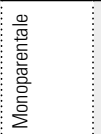 & 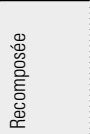 & 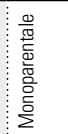 & 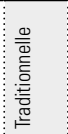 \\
\hline 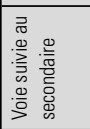 & $\ddot{m}$ & $\stackrel{m}{s}$ & $\stackrel{\circ}{p}$ & s & 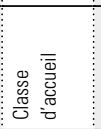 & $\stackrel{8}{p}$ & s & p & 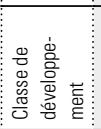 & $p$ & p & s \\
\hline 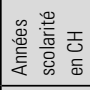 & 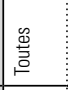 & ثِّ & م & : & 0 & 造 & 岕 & 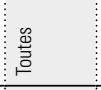 & 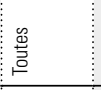 & 造 & 总 & 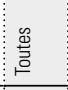 \\
\hline 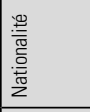 & 동 & 등 & 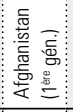 & $\begin{array}{l}\text { 등 } \\
\end{array}$ & 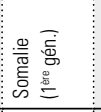 & 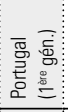 & 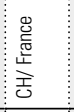 & I I & 덩 & 工 & I I & 工工 ㄱ \\
\hline \begin{tabular}{|l}
$\mathscr{\aleph}$ \\
$\tilde{\aleph}$ \\
\end{tabular} & $I$ & $I$ & $I$ & 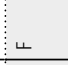 & $\leftarrow$ & \pm & I & 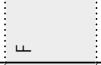 & $I$ & 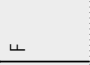 & $I$ & I \\
\hline 娄 & $=$ & 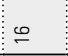 & $\stackrel{\circ}{\circ}$ & 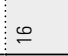 & $\mp$ & $\stackrel{\infty}{\simeq}$ & 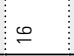 & $\stackrel{9}{\square}$ & $=$ & $\infty$ & 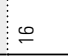 & 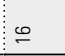 \\
\hline 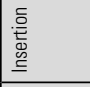 & 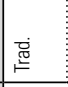 & 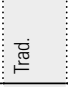 & 惡 & $\begin{array}{l}\text { 응 } \\
\text { 造 }\end{array}$ & 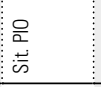 & 昰 & 焉 & 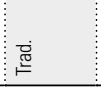 & 总 & 음 & 离 & 음 \\
\hline 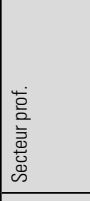 & 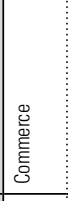 & 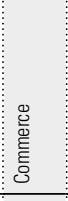 & 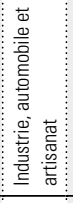 & 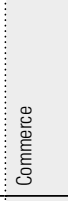 & 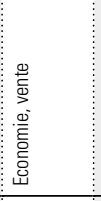 & 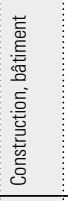 & 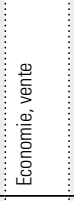 & : & 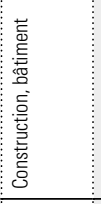 & 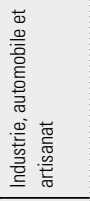 & 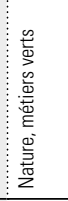 & 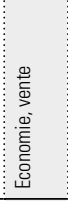 \\
\hline 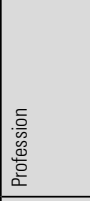 & 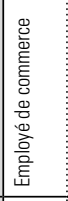 & 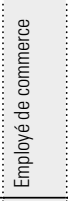 & 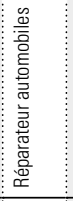 & 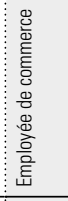 & 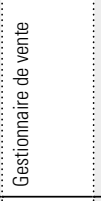 & 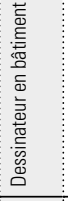 & 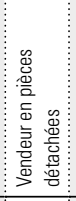 & 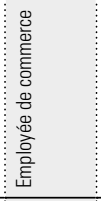 & 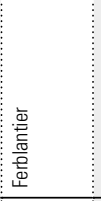 & 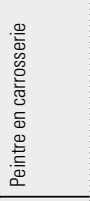 & $\begin{array}{l}\text { 衰 } \\
\text { 产 } \\
\text { 产 } \\
\text { 产 }\end{array}$ & 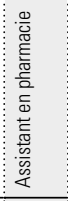 \\
\hline 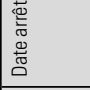 & 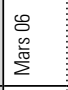 & \begin{tabular}{l}
8 \\
\multirow{\psi}{*}{}
\end{tabular} & $\begin{array}{l}8 \\
\overline{5} \\
\end{array}$ & $\begin{array}{r}8 \\
\stackrel{\Delta}{0} \\
\infty\end{array}$ & $\begin{array}{l}8 \\
\text { वे }\end{array}$ & $\begin{array}{l}8 \\
0 \\
8\end{array}$ & $\begin{array}{l}8 \\
2 \\
2\end{array}$ & $\begin{array}{l}8 \\
\stackrel{0}{0} \\
\infty\end{array}$ & $\begin{array}{l}8 \\
\text { o } \\
2\end{array}$ & $\begin{array}{l}8 \\
\dot{0} \\
\ddot{0}\end{array}$ & $\begin{array}{l}8 \\
8 \\
ٌ\end{array}$ & $\begin{array}{l}8 \\
2 \\
2\end{array}$ \\
\hline 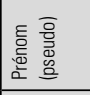 & 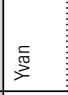 & 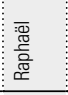 & 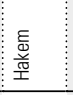 & 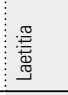 & 委 & $\frac{9}{3}$ & 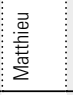 & 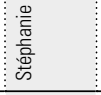 & $\frac{3}{\underline{\underline{x}}}$ & $\begin{array}{l}\infty \\
\text { 离 } \\
\end{array}$ & 兽 & 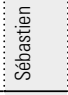 \\
\hline$\stackrel{2}{z}$ & $=$ & $\simeq$ & $\stackrel{m}{-}$ & \pm & $\stackrel{2}{\llcorner}$ & 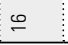 & $=$ & $\stackrel{\infty}{\rightleftharpoons}$ & 9 & i & $\bar{\lambda}$ & $\approx$ \\
\hline
\end{tabular}




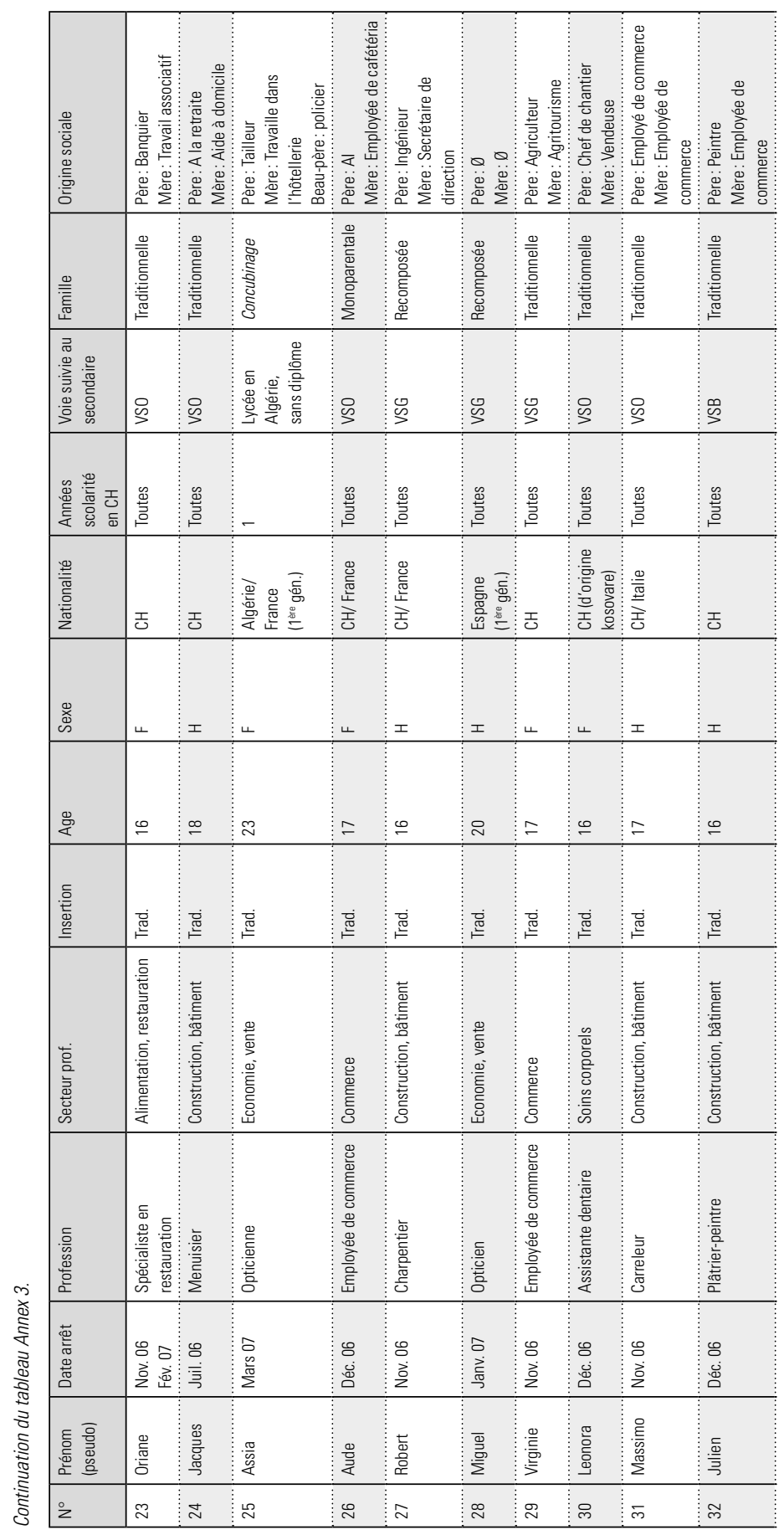

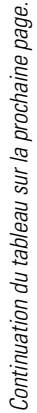




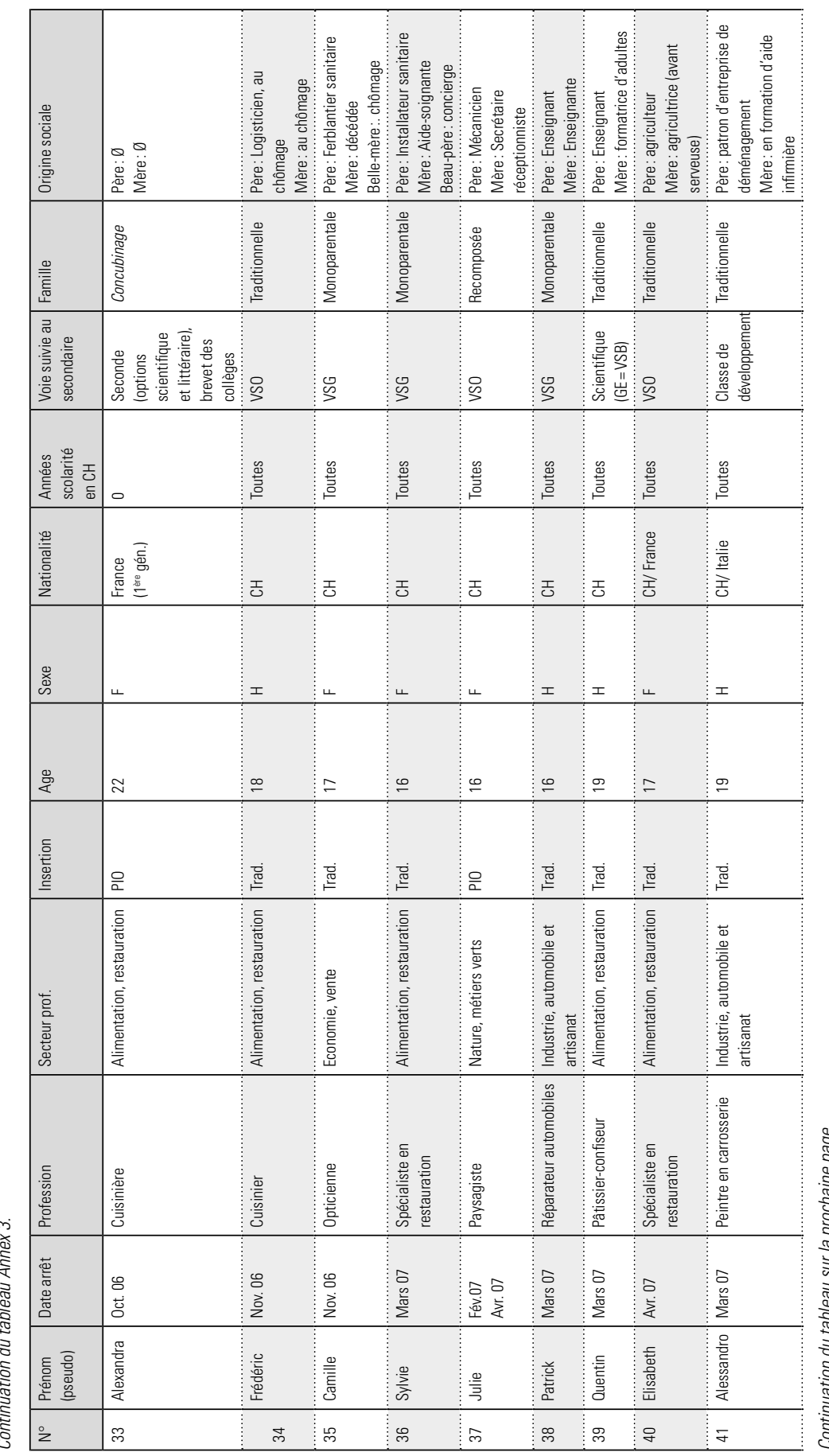




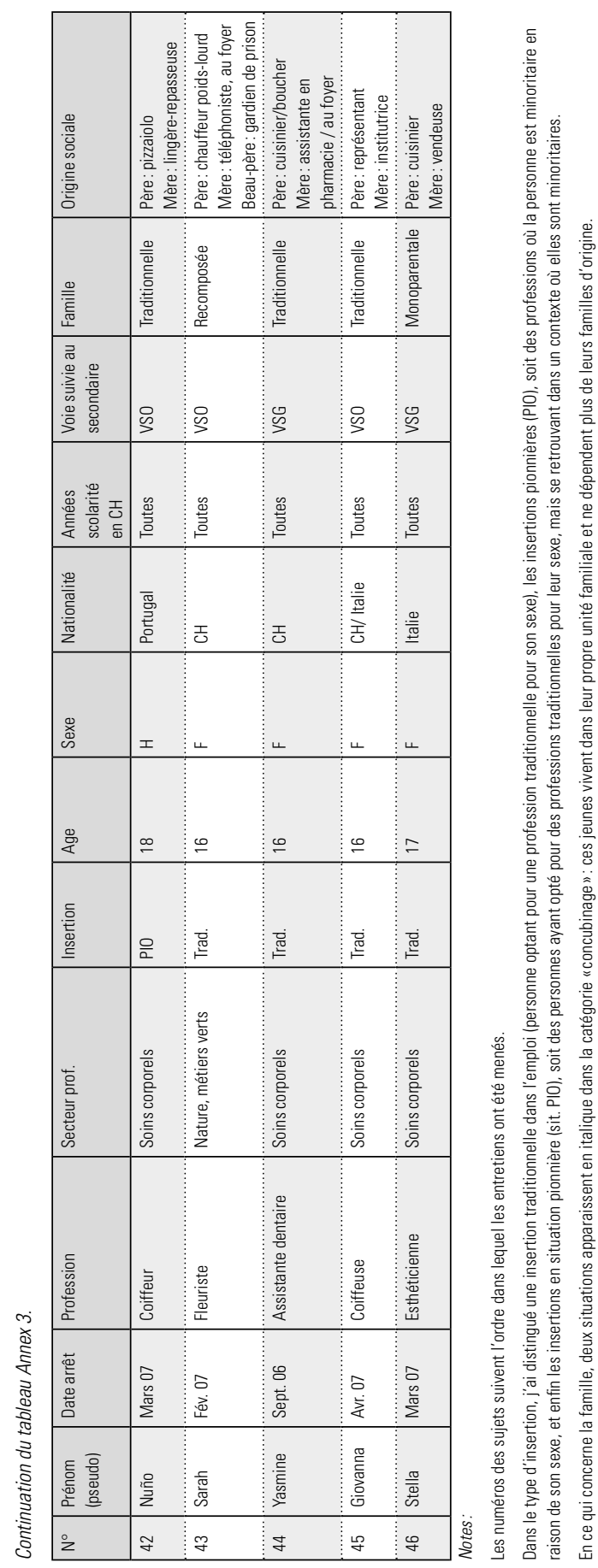




\section{Annexe 4 \\ Répartition par secteur professionnel et par sexe}

\begin{tabular}{lrrr}
\hline Secteur professionnel & Filles & Garçons & Total \\
\hline Alimentation, restauration & 4 & 2 & 6 \\
Commerce & 5 & 2 & 7 \\
Construction, bâtiment & 0 & 6 & 6 \\
Economie, vente & 3 & 5 & 8 \\
Industrie, automobile et artisanat & 1 & 6 & 7 \\
Nature, métiers verts & 5 & 1 & 6 \\
Soins corporels & 5 & 1 & 6 \\
Total & 23 & 23 & 46 \\
\hline
\end{tabular}

Si certains bastions apparaissent (métier de la construction et du bâtiment; industrie, automobile et artisanat; soins corporels), les chiffres ici ne donnent pas toujours une image exacte de la ségrégation en vigueur sur le marché du travail. Ces catégories sont en effet trop larges pour rendre compte de la ségrégation à l'intérieur des branches: en effet, si l'on prend les métiers verts: les paysagistes sont majoritairement des hommes, alors que l'horticulture est une profession davantage mixte et que les fleuristes sont en grande majorité des femmes. Pour les professions pionnières (voir Annexe 1).

\section{Annexe 5 \\ Répartition par catégorie socio-professionnelle d'origine et par sexe}

\begin{tabular}{lrrr}
\hline Catégorie socio-professionnelle & Femmes & Hommes & Total \\
\hline Travail non qualifié & 3 & 1 & 4 \\
Travail qualifié (manuel) & 4 & 3 & 7 \\
Emploi qualifié (non manuel) & 6 & 6 & 12 \\
Profession intermédiaire & 4 & 2 & 6 \\
Indépendant-e & 3 & 5 & 8 \\
Profession intellectuelle et d'encadrement & 1 & 5 & 3 \\
Autre* & 2 & 1 & 46 \\
Total & 23 & 23 & \\
\hline
\end{tabular}

Note:

La catégorie socio-professionnelle (CSP) des parents a été obtenue à partir des informations parfois lacunaires ou approximatives fournies sur la profession des parents, sans informations quant au revenu et au niveau de formation suivie. Celui-ci a donc, dans la mesure du possible, été déduit à partir de la profession exercée, avec des marges d'erreur quant aux trajectoires professionnelles et migratoires. Les catégories retenues sont basées sur la liste des professions de l'OFS, la situation dans la profession et lorsque nous avons pu la déduire, la formation suivie (Joye et al., 2007). Pour qualifier le niveau socio-professionnel du ménage ou famille d'origine, nous avons retenu la position parentale la plus élevée hiérarchiquement et l'avons attribuée au ménage (Levy, Joye, Guye et Kaufmann, 1998).

* Pas d'information ou situation hors travail (requérant.e.s d'asile interdite.es de travail). 
La formation professionnelle duale, au carrefour entre éducation et emploi, est représentative des tensions et enjeux propres à ces deux espaces. Cet ouvrage propose de la soumettre à une analyse de genre. Partant de la situation d'arrêts prématurés d'apprentissage, il questionne de manière plus large ce qui est produit et reproduit au cours de l'expérience apprentie. II interroge la façon dont la socialisation professionnelle participe d'une socialisation de genre. La formation professionnelle est ici analysée comme un lieu d'acculturation au travail, en particulier à l'un de ses piliers : la division sociale et sexuelle du travail. Cette grille de lecture donne l'occasion de réfléchir au statut des apprenti-e-s, à leur position particulière dans l'organisation du travail. L'analyse de cette « fabrique du genre » permet d'expliquer le maintien des inégalités de sexe. Par ses questionnements, ce livre fait intervenir la sociologie de l'éducation et du travail, ainsi que les études genre et interroge, au-delà des seuls arrêts prématurés, des enjeux centraux de la formation professionnelle suisse.

Nadia Lamamra est docteure ès sciences sociales de l'Université de Lausanne, où elle a enseigné les études genre. Elle est responsable du champ de recherche « Processus d'intégration et d'exclusion » à l'Institut fédéral des hautes études en formation professionnelle (EHB IFFP IUFFP). Elle est membre du comité de rédaction de la revue Nouvelles Questions Féministes. 